\title{
Multidimensional Contexts for Data Quality Assessment
}

by

\author{
Aida Malaki \\ A Thesis submitted to \\ the Faculty of Graduate Studies and Research \\ in partial fulfilment of \\ the requirements for the degree of \\ Master of Computer Science \\ in
}

\author{
Computer Science \\ Carleton University \\ Ottawa, Ontario, Canada
}

September 2012

Copyright (C)

2012 - Aida Malaki 
Library and Archives

Canada

Published Heritage

Branch

395 Wellington Street

Ottawa ON K1A ON4

Canada
Bibliothèque et

Archives Canada

Direction du

Patrimoine de l'édition

395 , rue Wellington

Ottawa ON K1A ON4

Canada
Your file Votre référence

ISBN: 978-0-494-94285-7

Our file Notre référence

ISBN: 978-0-494-94285-7
NOTICE:

The author has granted a nonexclusive license allowing Library and Archives Canada to reproduce, publish, archive, preserve, conserve, communicate to the public by telecommunication or on the Internet, loan, distrbute and sell theses worldwide, for commercial or noncommercial purposes, in microform, paper, electronic and/or any other formats.

The author retains copyright ownership and moral rights in this thesis. Neither the thesis nor substantial extracts from it may be printed or otherwise reproduced without the author's permission.
AVIS:

L'auteur a accordé une licence non exclusive permettant à la Bibliothèque et Archives Canada de reproduire, publier, archiver, sauvegarder, conserver, transmettre au public par télécommunication ou par l'Internet, prêter, distribuer et vendre des thèses partout dans le monde, à des fins commerciales ou autres, sur support microforme, papier, électronique et/ou autres formats.

L'auteur conserve la propriété du droit d'auteur et des droits moraux qui protege cette thèse. $\mathrm{Ni}$ la thèse ni des extraits substantiels de celle-ci ne doivent être imprimés ou autrement reproduits sans son autorisation.
In compliance with the Canadian Privacy Act some supporting forms may have been removed from this thesis.

While these forms may be included in the document page count, their removal does not represent any loss of content from the thesis.
Conformément à la loi canadienne sur la protection de la vie privée, quelques formulaires secondaires ont été enlevés de cette thèse.

Bien que ces formulaires aient inclus dans la pagination, il n'y aura aucun contenu manquant. 


\section{Abstract}

The notion of data quality cannot be separated from the context in which the data is produced or used. Recently, a conceptual framework for capturing context-dependent data quality assessment has been proposed.

According to it, the quality of a database $D$ is assessed with respect to a context which is modeled as an external system containing additional data, metadata, and definitions of quality predicates. The instance $D$ is 'put in context' via schema mappings; and after contextual processing of the data, a class of alternative clean versions $D^{\prime}$ of $D$ is produced. The quality of $D$ is measured in terms of its distance to this class.

In this work we extend contexts for data quality assessment by including multidimensional data, which allows to analyze data from multiple perspectives and different degrees of granularity. It is possible to navigate through dimensional hierarchies in order to go for the data that is needed for quality assessment.

More precisely, we introduce contextual hierarchies as components of contexts for data quality assessment. The resulting contexts are later represented as ontologies written in description logic. 
To my caring grandmother

To my loving niece 


\section{Acknowledgments}

I would like to thank my supervisor, Professor Leopoldo Bertossi for his support, advice and lots of good ideas throughout my thesis. His guidance and technical questions has been an eye opener for me on many occasions.

I also would like to thank Dr Flavio Rizzolo for his support and kind guidelines. Every meeting with him taught me a great deal and gave me new ideas.

I would like to thank my parents Nasrin and Reza, my elder sister Nazli for their unconditional love, support. I extend my appreciation to my eldest sister Nasim who was there whenever help was needed.

Last but not least, I would like to give special thanks to my friends, roommates, sisters Ava and Saba, for their great deal of support. Special thanks to Chris Rabbitt, my caring friend for his help for reviewing my thesis. Finally, I wish to thank my friends Mahkameh, Maryam, Sina, Zeinab and Mostafa, whom I have great memories with. 


\section{Table of Contents}

$\begin{array}{ll}\text { Abstract } & \text { ii }\end{array}$

Acknowledgments $\quad$ iv

Table of Contents $\quad$ v

List of Figures $\quad$ viii

List of Acronyms $\quad$ ix

1 Introduction 1

1.1 Contexts for Data Quality Assessment . . . . . . . . . . 3

1.2 Extending the Contextual Framework with Dimensions . . . . . . . . . 7

1.3 Ontological Representation of Contextual Dimensions . . . . . . . . . 11

1.4 Problem Statement and Contributions . . . . . . . . . . . . . 12

2 Preliminaries $\quad 15$

2.1 Databases and Integrity Constraints f . . . . . . . . . . . 15

2.1.1 Integrity Constraints .................. 15

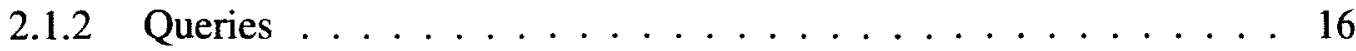

2.2 The Data Quality Assessment Framework . . . . . . . . . . . . 16

2.3 Multidimensional Databases ................... 21

2.4 Extended Entity-Relationship Model . . . . . . . . . . . . . . 23 


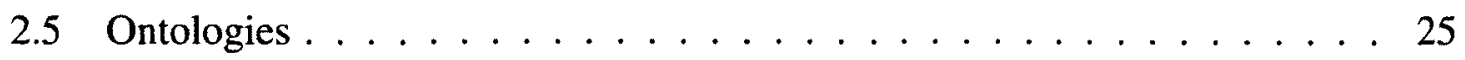

$2.5 .1 \quad$ Description Logics . . . . . . . . . . . . . . . . . 25

3 Extending the Contextual Framework with Dimensions 36

3.1 Facts Associating Contextual Dimensions $\ldots \ldots \ldots \ldots$

3.1.1 Categorical Relations . . . . . . . . . . . . . . . . . 37

3.1 .2 Attributive Relations . . . . . . . . . . . . . 38

3.2 Semantic Constraints . . . . . . . . . . . . . 40

3.2.1 Interdimensional Constraints . . . . . . . . . . . . 41

3.2.2 Intradimensional Constraints . . . . . . . . . . . . . 43

4 Ontological Representation of Contextual Dimensions 46

4.1 Translating a Contextual Dimension Schema into a DL TBox . . . . . . . 46

4.2 Translating a Contextual Dimension Instance into a DL ABox $\ldots \ldots . .52$

5 Ontological Representation of Semantic Constraints 55

5.1 Ontological Representation of Homogeneity . . . . . . . . . . . 55

5.2 Ontological Representation of Contextual Guidelines . . . . . . . . 56

5.3 Ontological Representation of Interdimensional Constraints . . . . . . . 58

5.4 Ontological Representation of Intradimensional Constraints . . . . . . . . 59

6 Experiments $\quad 64$

6.1 Introduction $\ldots \ldots \ldots \ldots \ldots \ldots \ldots \ldots \ldots \ldots \ldots \ldots \ldots \ldots \ldots$

6.2 Axioms in the TBox . . . . . . . . . . . . . 67

6.2.1 Representation of Semantic Constraints . . . . . . . . . 73

6.3 ABox Assertions . . . . . . . . . . . . . . 76

6.4 Data Quality Assessment . . . . . . . . . . . . . . . . . 79 
7 Conclusions and Future Work $\quad 85$

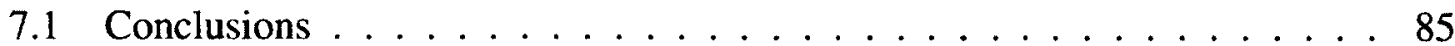

7.2 Future Work . . . . . . . . . . . . . . . 88

List of References

Appendix A OWL Representation of the Context 94

Appendix B Mappings from the Data Source to the Ontology 162 


\section{List of Figures}

1 Data Quality Assessment Framework . . . . . . . . . . . 7

$2 \quad$ PatienWard to Location Mapping . . . . . . . . . . . . . . . . . . . 8

$3 \quad$ Location Dimension Schema and an Instance $\ldots \ldots \ldots$

4 Attributive Relation HospitalDescription to Location Mapping and Categorial Relation UnitManuf to Location and Instrument Mappings . . . . . . 10

5 Using a Context for Quality Assessment . . . . . . . . . . . . . 21

6 Instrument Dimension Schema and an Instance $\ldots \ldots \ldots \ldots$

7 Entity Relationship Model . . . . . . . . . . . . . . . 24

8 Extended Entity Relationship Model . . . . . . . . . . . . . . 25

9 Linking Data to Ontologies . . . . . . . . . . . . . . 31

10 TomResults to Time Mapping . . . . . . . . . . . . . . . 38

11 HospitalFounded to Location and Time Mappings . . . . . . . . . . . . . . 58

12 Mapping a Data Source to an Ontology . . . . . . . . . . . 78

13 Ontology Satisfiability Verification . . . . . . . . . . . . 81 


\section{List of Acronyms}

\begin{tabular}{|c|c|c|c|}
\hline Acronyms & Definition & Acronyms & Definition \\
\hline ABox & Assertion Box & FOL & First Order Logic \\
\hline AR & Attributive Relation & GAV & Global As View \\
\hline CDT & Context Dimension Tree & GLAV & Global and Local As View \\
\hline CQP & Contextual Quality Predicate & $\mathrm{HM}$ & Hurtado-Mendelzon \\
\hline $\mathrm{CR}$ & Categorical Relation & IC & Integrity Constraint \\
\hline DAG & Directed Acyclic Graph & LAV & Local As View \\
\hline DB & Database & MDDB & Multidimensional Database \\
\hline $\mathrm{DL}$ & Description Logic & MDM & Multidimensional Model \\
\hline DQ & Data Quality & OBDA & Ontology Based Data Access \\
\hline DS & Contextual Dimension Schema & OLAP & Online Analytical Processing \\
\hline DWH & Data Warehouse & TBox & Terminological Box \\
\hline $\mathrm{E} / \mathrm{R}$ & Entity Relationship & VDIS & Virtual Data Integration Systems \\
\hline EER & Extended Entity Relationship & & \\
\hline
\end{tabular}




\section{Chapter 1}

\section{Introduction}

A model of context for data quality assessment is proposed in [15] [16], with the goal of formalizing the empirical fact that data quality is context dependent. In that work a context $\mathfrak{C}$ is represented as essentially a logical theory, into which a database $D$ under assessment can be mapped, for further processing and analysis. The instance $D$ is 'put in context' via schema mappings; and after contextual processing of the data, a class of alternative clean versions $D^{\prime}$ of $D$ is produced. The quality of $\mathrm{D}$ is measured in terms of its distance to this class. Metrics or distance measures can be introduced [15] [16].

More specifically, the contextual theories used in [16] appear as a form of data integration system, with additional (possibly partial) data, metadata, and definitions of quality predicates. The latter are used to extract quality data from those in the external dirty instance. In particular, the problem of doing clean query answering, i.e. of obtaining clean answers to queries posed to $D$ via the context $\mathfrak{C}$ was introduced and investigated.

An important element that was not included in those contexts is the one of data dimension. And this is necessary, because data for data quality analysis are almost always of a dimensional or hierarchical nature. Furthermore, dimensions provide different perspectives or points of view from which data can be seen and analyzed.

In this work we enrich our context model by introducing dimensions and their associated categories. The main purpose of this work is to convey the general ideas, problems, 
approach, and issues that appear when dimensions are introduced in combination with the other contextual elements mentioned above. For the same reason, we concentrate mainly on the introduction of dimensions, rather than on their use in data quality assessment.

To this end, we introduce in the context model an extension of the model for multidimensional databases (MDDBs), as represented in [37]. This model is extended with different kinds of relations associated to categories and dimensions (going beyond fact tables); and also with dimensional constraints.

Finally, following [15] [16], and in the spirit of having a context as a theory, we provide an ontological representation of the contexts as enriched with dimensions. We do this by using a description logic (DL) of the DL-Lite family [23], more specifically, DL-Lite ${ }_{\text {Horn }}^{(\mathcal{H})^{+}}$ [5]. The DL-Lite family provides a reasonable tradeoff between expressive power and complexity of query answering.

A context represented as an ontology will possibly admit several models in comparison to a context that is represented by a single relational database (ontologies are logical theories accepting several structures that make them true). As a consequence, logical reasoning, for example for checking inconsistencies, deriving implied relations, inferring instances of relationships and concepts, etc., becomes a new issue. From this point of view, a context becomes a knowledge base containing explicit data, e.g. in relational tables, explicit metadata, including data semantics, and also general rules that can be used for extracting implicit information.

In the rest of the Chapter 1 , we first present the notion of data quality. Then, we show how contexts (in particular the multidimensional contexts) can be used in assessing the quality of data. Later on in this chapter, we briefly demonstrate the ontological representation of contexts. 


\subsection{Contexts for Data Quality Assessment}

The quality of data is relative to their intended and interpreted use [38]. It is related to the possible differences between the actual stored values and the real values that were expected or supposed to be stored [16]. Furthermore, the notions of 'good' and 'poor' data quality are inseparable from the context in which the data is used or produced [16].

In this thesis, data quality (DQ) is addressed from these points of view, i.e. in relation to semantic discrepancy [38] (as opposed to misspellings, for example), and as determined by a formal context that enables data quality analysis.

Example 1.1. Tom is a patient in a hospital. Several times a day different medical tests are performed on him, and test values are recorded by a nurse. His doctor, John, wants to see Tom's test values every day, to follow his evolution.

The data that John needs about Tom appear, among other, in the PatientValue relation in Table 1.1. John has additional quality concerns. He only wants to see, for all his patients, test results that are taken with instruments of the brand $B_{1}$. On Sep/5, for morning tests, the nurse Jane performed the test on Lou with an instrument of brand $B_{2}$, and inserted it as the 6th tuple into the PatientValue relation.

\begin{tabular}{|c|c|c|}
\hline \multicolumn{3}{|c|}{ Table 1.1: Patient Value } \\
\hline Patient & Value & Time \\
\hline Tom Waits & 38.5 & $11: 45 / 5 /$ Sep/2011 \\
Tom Waits & 38.2 & $12: 10 / 5 /$ Sep/2011 \\
Tom Waits & 38.1 & $11: 50 / 6 /$ Sep/2011 \\
Tom Waits & 38.0 & $12: 15 / 7 /$ Sep/2011 \\
Tom Waits & $110 / 70$ & $11: 45 / 8 /$ Sep $/ 2011$ \\
Lou Reed & 37.9 & $12: 10 / 5 /$ Sep/2011 \\
\hline
\end{tabular}

Based on John's quality concerns, this tuple should not appear in a quality relation, one that satisfies John's requirements. However, it does appear in the doctor's view of data (which is Table 1.1). In this case, there is a difference between the value which is recorded and the real value which was expected to be recorded (one measured with an instrument from the intended brand). This is an example of 'semantic discrepancy' or 'semantically inaccurate data' [10]. 
The quality of data depends on the context [16]. In this work, we define a context for the quality assessment of a database instance $D$ of schema $\mathcal{S}$ as a separate information system $\mathfrak{C}$. The latter may contain its own relational schema $\mathcal{C}$, a possibly partial (incomplete) instance $I$ of $\mathcal{C}$, additional predicates with definitions in $\mathfrak{C}$ that can be used for $D$ 's quality assessment, etc.

The schema $\mathcal{C}$ could be an extension of $\mathcal{S}$, and $I$ an extension of $D$. In order to assess the quality of $D$, the latter has to be put in context via schema mappings between $\mathcal{S}$ and $\mathcal{C}$. Different cases and situations can be accommodated in this framework [16]. A quality database instance $D^{\prime}$, as an alternative to the instance $D$ at hand, could be a footprint of the contextual instance $I$ after some additional processing via quality predicates at the contextual level. Depending on how much $D$ departs from $D^{\prime}$, we can assign to the data in $D$ a quality grade or measure. In other cases, instead of a single quality instance $D^{\prime}$, we can obtain a whole class $\mathcal{K}$ of quality instances, and $D$ has to be assessed on the basis of its distance to $\mathcal{K}[16]$.

We can see that a context for data quality assessment can be conceived as a shared information space that is designed to serve a particular purpose [27].

Example 1.2. (example 1.1 cont.) We have a contextual relation Measurement (Table 1.2). Measurement contains all the values of different tests that are performed on patients by using instruments of different brands at different times. From relation Measurement we obtain the values that are taken by instruments of brand $B_{1}$, satisfying the doctor's requirement. 


\begin{tabular}{|c|c|c|c|}
\hline \multicolumn{4}{|c|}{ Table 1.2: Measurement } \\
\hline Patient & Value & Hour & Brand \\
\hline Tom Waits & 38.5 & $11: 45 / 5 / \mathrm{Sep} / 2011$ & $\mathrm{~B}_{1}$ \\
Tom Waits & 38.2 & $12: 10 / 5 / \mathrm{Sep} / 2011$ & $\mathrm{~B}_{1}$ \\
Tom Waits & 38.1 & $11: 50 / 6 / \mathrm{Sep} / 2011$ & $\mathrm{~B}_{1}$ \\
Tom Waits & 38.0 & $12: 15 / 7 / \mathrm{Sep} / 2011$ & $\mathrm{~B}_{1}$ \\
Tom Waits & $110 / 70$ & $11: 45 / 8 / \mathrm{Sep} / 2011$ & $\mathrm{~B}_{3}$ \\
Lou Reed & 37.9 & $12: 10 / 5 / \mathrm{Sep} / 2011$ & $\mathrm{~B}_{2}$ \\
\hline
\end{tabular}

\begin{tabular}{|c|c|c|}
\hline \multicolumn{3}{|c|}{ Table 1.3: PatientValue' } \\
\hline Patient & Value & Time \\
\hline Tom Waits & 38.5 & $11: 45 / 5 / \mathrm{Sep} / 2011$ \\
Tom Waits & 38.2 & $12: 10 / 5 / \mathrm{Sep} / 2011$ \\
Tom Waits & 38.1 & $11: 50 / 6 / \mathrm{Sep} / 2011$ \\
Tom Waits & 38.0 & $12: 15 / 7 / \mathrm{Sep} / 2011$ \\
\hline
\end{tabular}

The quality version of PatientValue relation based on John's condition is Table 1.3. This new instance is obtained via a selectproject view on Measurement at the contextual level. The quality of Table 1.1 is assessed by comparing it with the extension of PatientValue' in Table 1.3 by using some appropriate distance measure [16].

The context $\mathfrak{C}$ could also contain additional metadata or general knowledge that can be used in data quality assessment.

Example 1.3. (example 1.2 cont.) Let us assume that, instead of Table 1.2, we have the table PatientWard (Table 1.4). It contains all the patients and the wards they were staying 
on each day. Note that it does not explicitly contain information about the tests or the instruments used. However, the context has information about hospital guidelines:

Contextual Hospital Guideline 1: 'Medical tests on patients in ward $\mathrm{W}_{1}$ have to be performed by instruments of brand $B_{1}$ '.

A contextual guideline like this can be used in different forms, e.g. as a hard rule, as a default rule, or as a semantic constraint at the contextual level.

\begin{tabular}{|c|c|c|}
\hline \multicolumn{3}{|c|}{ Table 1.4: PatientWard } \\
\hline Patient & Date & Ward \\
\hline Tom Waits & $5 /$ Sep/2011 & $\mathrm{W}_{1}$ \\
Tom Waits & $6 / \mathrm{Sep} / 2011$ & $\mathrm{~W}_{1}$ \\
Tom Waits & $7 / \mathrm{Sep} / 2011$ & $\mathrm{~W}_{1}$ \\
Lou Reed & $5 / \mathrm{Sep} / 2011$ & $\mathrm{~W}_{2}$ \\
\hline
\end{tabular}

If we had the contextual instance of Table 1.2 with the detailed measurements, and assuming that the contextual guideline is satisfied, we can use the contextual relation PatientWard in combination with the contextual hospital guideline to conclude that all tests performed on Tom Waits between $5 /$ Sep and $7 / \mathrm{Sep}$, the dates in which he was

in Ward 1, were done with instruments of brand $\mathrm{B}_{1}$. The quality version of PatientValue relation is again Table 1.3.

The framework for data quality assessment proposed in [16] is depicted in Figure 1. It shows the relational schema $\mathcal{S}$ with its predicates $R_{1}, R_{2}, \ldots, R_{n}$. The instance $D$ of $\mathcal{S}$ under quality assessment has extensions $R_{1}(D), \ldots, R_{n}(D)$ for predicates. Context $\mathfrak{C}$ contains a contextual schema, $\mathcal{C}$, including a set $\mathcal{B}$ of built-in predicates, e.g. comparisons; and also a set, $\mathcal{P}=\left\{P_{1}, \ldots, P_{k}\right\}$, of contextual quality predicates (CQPs) with definitions over $\mathfrak{C}$.

The connection between the schemas in the framework is provided by schema mappings [12], $\alpha_{i}$, like those found in virtual data integration systems (VDISs) [13] [42] or data exchange [4]. Now, schema $\mathcal{S}^{\prime}$ is a copy of schema $\mathcal{S}$, with relational predicates $R_{1}^{\prime}, \ldots, R_{n}^{\prime}$. Their extensions are the ideal, quality instances for the $R_{i}^{\prime} \mathrm{s}$. Each of the $R_{i}^{\prime} \mathrm{s}$ is defined as 


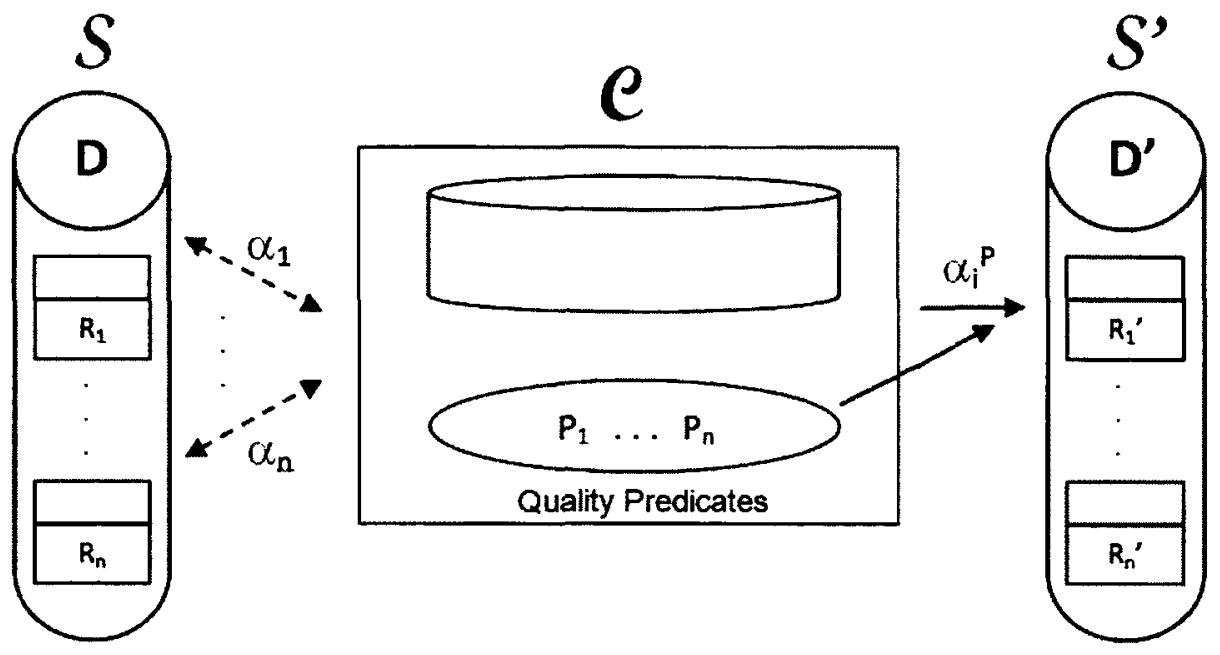

Figure 1: Data Quality Assessment Framework

a conjunctive view over the contextual schema, say $\forall \bar{x}\left(\alpha_{i}^{\mathcal{P}}(\bar{x}) \equiv R_{i}^{\prime}(\bar{x})\right)$, where $\alpha_{i}^{\mathcal{P}}$ is a conjunction of atomic formulas with predicates in $\mathcal{C} \cup \mathcal{P}$. These views are computed on top of contextual instances $I$ for schema $\mathcal{C}$, that are related to $D$ in terms of contents by the mappings $\alpha_{i}$. In this way, $D$ is mapped into $\mathfrak{C}$, integrated into contextual instances $I$, and further qualified via the views $R_{i}^{\prime}$, to obtain quality data [16].

\subsection{Extending the Contextual Framework with Dimensions}

In Example 1.2, the required data was explicitly stored in a relation that extends the table under assessment. However, in some other situations we may have to go outside a contextual table and navigate within the context, searching for the necessary data. This is particularly the case when the contextual data is of a multidimensional and hierarchical nature [19] [20]. See Section 2.3 for details.

Example 1.4. (example 1.2 cont.) John has a new quality requirement. He asks nurses to perform all the medical tests with instruments made by manufacturer $\mathbf{M}_{1}$. Furthermore, there is a new contextual hospital guideline in place:

Contextual Hospital Guideline 2: 'Medical tests on patients in standard care unit has 


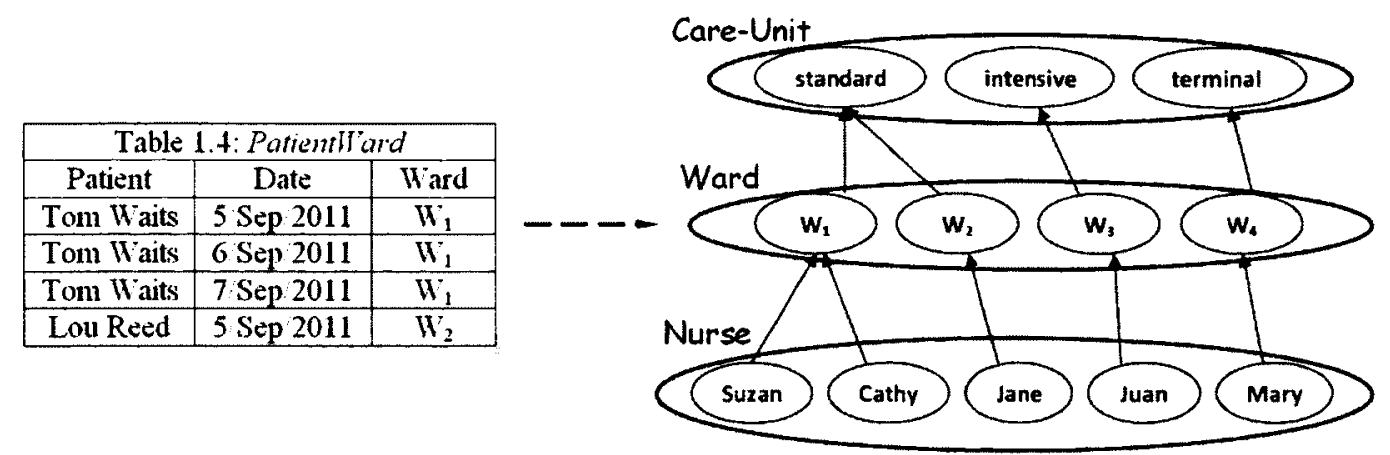

Figure 2: PatienWard to Location Mapping

to be taken with instruments made by manufacturer $\mathrm{M}_{1}$ '.

The information explicitly provided by the contextual relation PatientWard (Table 1.4) is about the Ward category, but data about the Care Units, that could be used in combination with Guideline 2, belongs to a higher or more general category, Care-Unit. This is illustrated in Figure 2.

\begin{tabular}{|c|c|c|}
\hline \multicolumn{3}{|c|}{ Table 1.5: PatientValue" } \\
\hline Patient & Value & Hour \\
\hline Tom Waits & 38.5 & $11: 45 / 5 / \mathrm{Sep} / 2011$ \\
Tom Waits & 38.2 & $12: 10 / 5 / \mathrm{Sep} / 2011$ \\
Tom Waits & 38.1 & $11: 50 / 6 / \mathrm{Sep} / 2011$ \\
Tom Waits & 38.0 & $12: 15 / 7 / \mathrm{Sep} / 2011$ \\
Lou Reed & 37.9 & $12: 10 / 5 / \mathrm{Sep} / 2011$ \\
\hline
\end{tabular}

In order to reach the relevant data, we have to navigate through the hierarchy. In this case, by rolling up from Ward to Care-Unit, we can identify the wards that belong to standard care units, namely $\mathrm{W}_{1}$ and $\mathrm{W}_{2}$. This allows us to build a quality version of the original instance, the one shown in Table 1.5.

Data in a contextual hierarchy are organized in categories that are (possibly partially) ordered according to the level of detail they provide. A contextual hierarchy provides information from one perspective according to different degrees of details. 


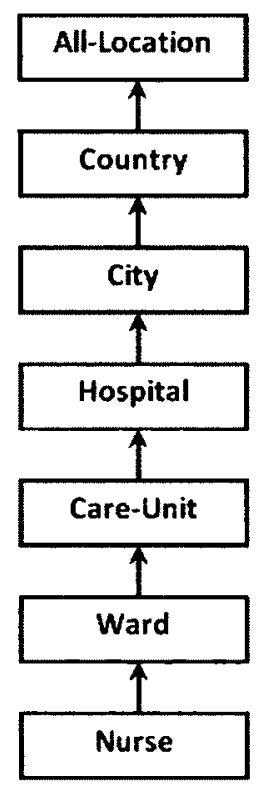

(a) Schema

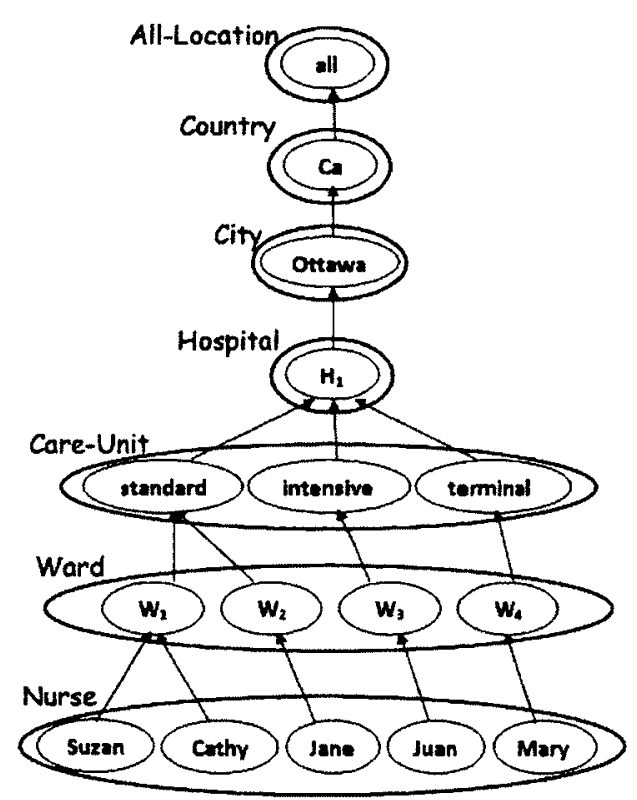

(b) Instance

Figure 3: Location Dimension Schema and an Instance

By having several hierarchies in a context, we will have multiple perspectives for data analysis and quality assessment.

Example 1.5. The hierarchy in Figure 2 is a portion of the Location hierarchy (or dimension) in Figure 3. The dimension schema in Figure 3a shows the categories and their relationships. Figure 3b shows a possible dimension instance for the Location; and a partial order relation between the elements of the categories.

Dimensions can be made part of a context by embedding in it a multidimensional database. For this purpose we can use an extension of the Hurtado-Mendelzon (HM) data model for multidimensional databases (MDDBs) [37]. The HM data model for multidimensional databases (MDDBs) is described in details in Section 2.3.

As is common in DWHs and MDDBs, in general, we can have fact tables associated to the base categories. However, using a multidimensional model and a multidimensional database within a context serves purposes not traditionally found in data warehousing, 


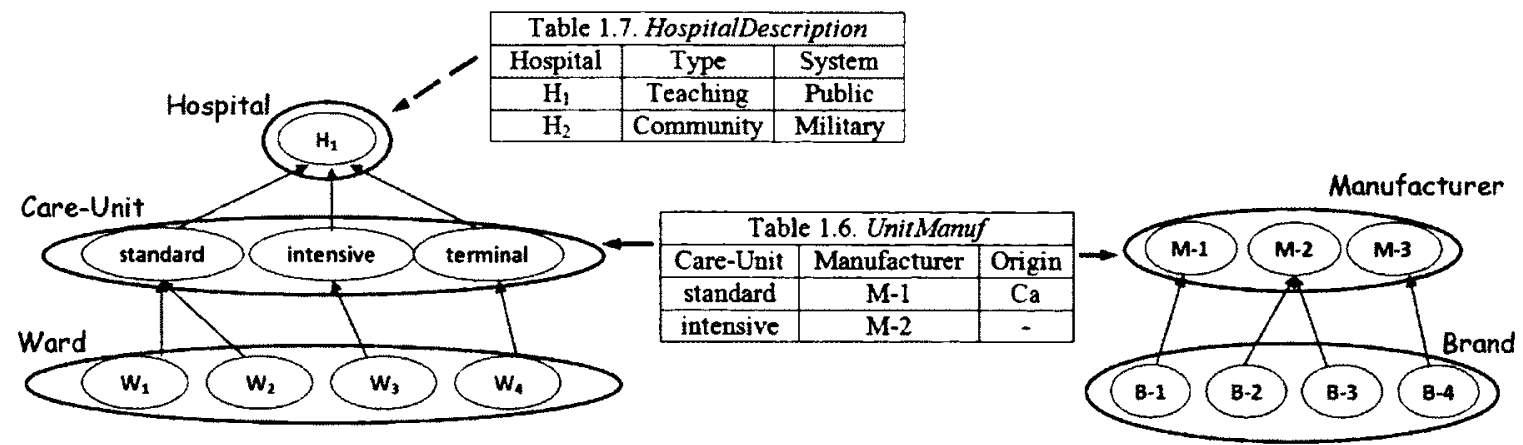

Figure 4: Attributive Relation HospitalDescription to Location Mapping and Categorial Relation UnitManuf to Location and Instrument Mappings

OLAP, or data analytics. We can, for example, extend the HM model with additional tables associated to the different level of the hierarchies or to categories other than base categories (to which the fact tables are usually associated).

Example 1.6. (example 1.4 cont.) In Figure 4, we have two kinds of tables associated to categories. Relation HospitalDescription (Table 1.7) is connected only to the Hospital category, providing descriptions for the elements of the category. In contrast, relation UnitManuf (Table 1.6) represents the Guideline 2 and contains attributes connecting two categories, Care-Unit and Manufacturer, in different dimensions, say Location and Instrument. It contains an extra, non-dimensional attribute Origin.

A table like UnitManuf can be used in combination with dimensional navigation to obtain the required data; in this case about the instrument used with the patients of a given care unit. Notice that the table might be incomplete: not all care units or manufactures are necessarily related.

In more general terms, we extend the multidimensional model (MDM) with categorical relations (e.g. relation UnitManuf (Table 1.6)) and attributive relations (e.g. relation HospitalDescription (Table 1.7)), both connected to categories of dimension hierarchies. 
See Chapter 3 for the full discussion.

Introducing the extended MDM in the context brings up the many other sensible elements to be considered, in particular, intradimensional and interdimensional constraints. These constraints restrict combinations of values in the relations associated to the different level of the hierarchies, or pair of them. As an example a constraint for specifying the commonsense assumption that no single measurement can be taken by more than one nurse. These constraints are introduced in Chapter 3.

In this section we have motivated how contexts can be enriched by the introduction of multidimensional models. The basis can be a model for MDDBs, like the one in [37]. However, it can be extended with additional data associated to category elements. On the other side, a given contextual relation can be linked to one or several dimension instances. Navigation through them allows us to find explicit additional information or implicit information, like the one provided by contextual guidelines, as rules in the contextual framework. Navigation is a process that could be realized with the machinery developed in [43] [44], that is an extension of relational algebra, denoted as Contextual Relational Algebra (CRA), for querying data at different levels of details.

\subsection{Ontological Representation of Contextual Dimensions}

A context could be a whole ontology, i.e. a knowledge base, containing explicit data, e.g. in relational tables, explicit metadata, including data semantics, and also general rules that can be used for extracting implicit information. This would be the case, for example, of the contextual hospital guidelines of the previous sections. An ontology would allow us to extract and analyze information via some sort of logical reasoning.

Ontologies are written in different languages such as OWL [46], DL [8], etc. In this thesis, with the motivation of having a full-fledged ontology as a contextual framework for data quality assessment [16], we will show how the dimensions (and their extensions) can 
be expressed as a part of an ontology in a description logic (DL).

In DL there is knowledge both at the intentional level, in a terminological TBox, $\mathcal{T}$, and at the extensional level, in an assertional $\mathrm{ABox}, \mathcal{A}$. The TBox describes the vocabulary of an application domain, while the ABox consists of the assertions about named individuals in terms of this vocabulary. The vocabulary consists of concepts and roles. Concepts are unary predicates, which denote sets of individuals. Roles are binary predicates, which denote binary relationships between individuals. Concepts and roles can be atomic or complex. See Section 2.5.1 for details.

In this thesis, we sketch a DL-based representation of the extended MDM in one of the members of $D L$-Lite family [23]. In general, DL-Lite and its extensions have a good balance of expressive power and good computational properties; and have found interesting applications in data management and semantic web [24].

In Chapter 4 we show how the contextual relational schema and the contextual dimension schemas become part of a TBox $\mathcal{T}$. In addition, we show how the instances of contextual dimensions, categorical and attributive relations are mapped to the corresponding constructs in $\mathcal{T}$ (concepts and roles). In Chapter 5, we discuss about the ontological representation of the semantic constraints over the extended HM model.

\subsection{Problem Statement and Contributions}

The goal of this research is to motivate, formalize and investigate the notion of data quality assessment. We believe that data quality assessment is a context dependent activity. The key features of our research are the following:

1. We introduce multidimensional data as a part of the context. This allows to analyze data from multiple perspectives and different degrees of details. It is possible to navigate through dimensional hierarchies in order to go for the data that is needed for 
quality assessment. In addition, we extend the HM data model with two new relations, categorical relations and attributive relations. They are associated to different levels of the hierarchies or to categories other than base categories. We also show the relevance of introducing and having intra- and interdimensional semantic constrains. They restrict certain combinations of related values that may appear in (or are associated to) different categories in a dimension, or pair of them. The extended MDM is embedded into the context after reconstructing the context as a DL ontology [8].

2. We illustrate an ontological representation of the contexts as enriched with dimensions. An ontology provides us with the capability of performing different kinds of logical reasoning for extracting and analyzing information. An ontology offers language components and constructs for representing knowledge about a domain of interest. More concretely, we represent contexts as ontologies written in a description logic of the DL-Lite family [23], actually, DL-Lite $e_{\text {Horn }}^{(\mathcal{H N})^{+}}$[5]. In the spirit of having a full-fledge ontology as a contextual framework for data quality assessment [16], we show how the extended MDM can be expressed as a part of an ontology in $D L$ Lite $_{\text {Horn }}^{(\mathcal{H} N)^{+}}$.

This thesis is structured as follows: In Chapter 2, we review technical preliminaries. In Chapter 3, we show how contexts can be enhanced by introducing contextual dimensions. In Chapter 3, we extend the HM model with two types of the contextual relations which are connected to the different levels of the contextual dimensions, namely 'categorical relations' and 'attributive relations'. We also introduce the semantic constraints over these relations.

In Chapter 4, we describe the translation of the contextual dimensions into a suitable description logic theory. Chapter 5 shows how to represent semantic constraints over the extended HM model in the DL ontology. Chapter 6 shows an experiment on how the proposed ontological representation of the context is used for obtaining the quality instances. 
In Chapter 7 we draw conclusions and discuss future works. 


\section{Chapter 2}

\section{Preliminaries}

\subsection{Databases and Integrity Constraints}

In the context of relational databases, we assume that we have a fixed relational schema $\Sigma=$ $(\mathcal{U}, \mathcal{R}, \mathcal{B})$, where $\mathcal{U}$ is the possibly infinite database domain, $\mathcal{R}$ is a fixed set of database predicates $\mathcal{R}=\left\{R_{1}, R_{2}, R_{3}, \ldots\right\}$, where each relation $R$ has a finite, ordered set of attributes $\mathcal{A}_{R} ;$ and $\mathcal{B}$ is a fixed set of built-in predicates, like comparison predicates, e.g.: > ,$<,=R[i]$ denotes the attribute in position $i$ of predicate $R \in \mathcal{R}$. The schema determines a language $\mathcal{L}(\Sigma)$ of first-order predicate logic. A database instance $D$ compatible with $\Sigma$ can be seen as a finite collection of ground atoms (or tuples) of the form $R\left(c_{1}, \ldots, c_{n}\right)$, where $R$ is a predicate in $\mathcal{R}$ and the $c_{1}, \ldots, c_{n}$ are constants in $\mathcal{U}$. Built-in predicates have a fixed extension in every database instance.

\subsubsection{Integrity Constraints}

Integrity constraints (ICs) have been considered in the relational databases for adding semantics, and ensuring accuracy and consistency of data.

Integrity constraints are closed first-order formulas (i.e. sentences) of the language $\mathcal{L}(\Sigma)$. We assume that sets of integrity constraints ICs are logically consistent as sets of sentences of $\mathcal{L}(\Sigma)$. In Chapter 3, we use the following classes of integrity constraints: 
Definition 1. If $\bar{x}_{1}, \ldots, \bar{x}_{n}$ represents tuples of variables and constants, and $B \in \mathcal{B}$, a denial integrity constraint $[17]$ is a sentence in $\mathcal{L}(\Sigma)$ of the form:

$$
\forall \bar{x}_{1}, \ldots, \bar{x}_{n} \cdot \neg\left[\bigwedge_{i=1}^{n} R_{i}\left(\bar{x}_{i}\right) \wedge B\left(\bar{x}_{1}, \ldots, \bar{x}_{n}\right)\right] .
$$

The interdimensional constraint in Example 3.6 is an example of denial integrity constraint.

Definition 2. A referential integrity constraint [17] is a sentence in $\mathcal{L}(\Sigma)$ of the form:

$$
\forall \bar{x}(P(\bar{x}) \rightarrow \exists \bar{z} Q(\bar{x}, \bar{z})
$$

where $P, Q \in \mathcal{R}$.

The intradimensional constraint in Example 3.8 is an example of referential integrity constraint.

\subsubsection{Queries}

A query is a first-order formula over the language $L(\Sigma)$. We restrict ourselves to the class of conjunctive queries.

Definition 3. A conjunctive query $\mathcal{Q}(\bar{x})(\mathrm{CQ})$ is of the form:

$$
\exists \bar{y}\left(\bigwedge_{i=1}^{n} R_{i}\left(\bar{x}_{i}, \bar{y}_{i}\right) \wedge \varphi\right)
$$

where $R_{i} \in R, \varphi$ is a conjunction of built-ins whose variables appear in the $R_{i} \mathrm{~s}$, and $\bar{x}=\bigcup_{i} \bar{x}_{i}$ which are the free variables of the query.

\subsection{The Data Quality Assessment Framework}

A framework for assessing the quality of data is proposed in [16]. As illustrated in Figure 1 , the framework consists of a relational schema $\mathcal{S}$ which contains relational predicates 
$R_{1}, R_{2}, \ldots, R_{n} \in \mathcal{S}$. An instance $D$ of the schema $\mathcal{S}$ with extensions $R_{1}(D)$ for $R_{1}, \ldots$, $R_{n}(D)$ for $R_{n}$ is under quality assessment. $D$ is put into a context $\mathfrak{C}$ (including built-ins) via some mappings (the $\alpha_{i}$ in Figure 1). The data in $D^{\prime} s$ image in $\mathfrak{C}$ are combined with additional information existing in $\mathfrak{C}$. This additional information can be local data at $\mathfrak{C}$, definitions of quality predicates and additional semantic constraints.

The participating schemas are related by schema mappings [12] like those in data exchange [4] or virtual data integration systems (VDIS)s [13] [42]. A common form of association, or mapping, is of the form $\forall \bar{x}\left(S(\bar{x}) \rightarrow \varphi_{G}(\bar{x})\right)$, where $S$ is a relational predicate of a data source and $\varphi_{G}(\bar{x})$ is a conjunctive query over a global relational schema $G$. These association can be found in local- as-view (LAV) VDISs with open (or sound) sources. Another common form of association is of the form $\forall \bar{x}\left(\psi_{S}(\bar{x}) \rightarrow G(\bar{x})\right)$, found in global-as-view (GAV) VDISs with open sources, where $\psi_{R}(\bar{x})$ is a conjunctive query over the union $R$ of the relational schemas at the sources, and $G$ is a global relational predicate. In global-and-local-as-view (GLAV) VDISs with open sources, we find associations between views (or queries), of the form $\forall \bar{x}\left(\psi_{R}(\bar{x}) \rightarrow \varphi_{G}(\bar{x})\right.$ ).

In particular, the data source under quality assessment $D$ may be mapped into the contextual schema $\mathcal{C}$. A common form of mapping is:

$$
\alpha_{R}: \forall \bar{x}\left(R(\bar{x}) \rightarrow \varphi^{\mathcal{C}}(\bar{x})\right),
$$

where $\mathrm{R} \in \mathcal{S}$ and $\varphi^{\mathcal{C}}(\bar{x})$ is a conjunctive query over schema $\mathcal{C}$.

Example 2.1. Assume the relation PatientValue (Table 1.1) is under quality assessment and the relation Measurement (Table 1.2) is a contextual relation. Based on (1), a mapping between the PatientValue schema and the Measurement schema is defined as:

$$
\forall p \forall v \forall t(\text { PatientValue }(p, v, t) \rightarrow \text { Measurement }(p, v, t, b))
$$

In the data quality assessment framework in Figure 1, the schema $\mathcal{S}^{\prime}$ is a copy of the 
schema $\mathcal{S}$ with relational predicates $R_{1}^{\prime}, \ldots, R_{n}^{\prime}$. Each of the $R_{i}^{\prime}$ is a quality version of the $R_{i}$ in the schema $\mathcal{S}$.

In addition to the contextual schema $\mathcal{C}$, we may have a set $\mathcal{P}$ of contextual quality predicates (CQP)s with definitions inside $\mathcal{C}$. CQPs could be defined entirely in terms of (or as views over) schema $\mathcal{C}$. However, we keep them separate to emphasize their role in expressing data quality concerns. We obtain a combined contextual schema $\mathcal{C} \cup \mathcal{P}$. Typically, each $\mathrm{P} \in \mathcal{P}$ is defined as a conjunctive view:

$$
P(\bar{x}) \leftarrow \gamma^{\mathcal{C}}(\bar{x})
$$

in terms of elements in $\mathcal{C}$ (and possibly built-in predicates). Each CQP stands for an atomic quality requirement requested by a data consumer or met by a data producer. Defining CQPs over context, restricts the admissible values for certain attributes in tuples.

Example 2.2. Assume in addition to the contextual relation Measurement, also the context $\mathcal{C}$ contains the following contextual relations:

\begin{tabular}{|c|c|c|c|c|c|}
\hline \multicolumn{3}{|c|}{ Table 2.1: Assign } & \multicolumn{3}{|c|}{ Table 2.2: Registration } \\
\hline Nurse & Hour & Patient \\
Suzan & $11: 45 / 5 / \mathrm{Sep} / 2011$ & Tom Waits & Nurse & Registration \\
\hline Cathy & $12: 10 / 5 / \mathrm{Sep} / 2011$ & Tom Waits & Cathy & 1997 \\
Suzan & $11: 50 / 6 / \mathrm{Sep} / 2011$ & Tom Waits & Mary & 2000 \\
Cathy & $12: 15 / 7 / \mathrm{Sep} / 2011$ & Tom Waits \\
Suzan & $11: 45 / 8 / \mathrm{Sep} / 2011$ & Tom Waits \\
Jane & $12: 10 / 5 / \mathrm{Sep} / 2011$ & Lou Reed \\
\hline
\end{tabular}


John - doctor - in Example 1.1 wants to see only test results that are taken by a registered nurse. Based on (2), a quality predicate Registered is defined over the contextual relations Assign and Registration as:

$$
\text { Registered }(t, p) \leftarrow \text { Assign }(n, t, p), \text { Registration }(n, r)
$$

The quality extension of predicate $R \in \mathcal{S}$ is obtained as the extension of a new predicate $R_{p}^{\prime}$ through the following mapping:

$$
\alpha_{R}^{\mathcal{C}, \mathcal{P}}: \forall \bar{x}\left(\imath^{\prime \mathcal{C} . \mathcal{P}}(\bar{x}) \rightarrow R_{\mathcal{P}}^{\prime}(\bar{x})\right)
$$

where $\psi^{\mathcal{C}, \mathcal{P}}$ is a conjunctive query over schema $\mathcal{C} \cup \mathcal{P}$. Notice that $\mathcal{C}$ contains schema $\mathcal{S}^{\prime}$.

A special and common case corresponds to definitions via conjunctive views of the form:

$$
\alpha_{R}^{\mathcal{C}, \mathcal{P}}: R_{\mathcal{P}}^{\prime}(\bar{x}) \leftarrow \varphi_{R}^{\mathcal{C}}\left(\bar{x}_{1}\right), \varphi_{R}^{\mathcal{P}}\left(\bar{x}_{2}\right)
$$

where $\bar{x} \subseteq \bar{x}_{1} \cup \bar{x}_{2}$, and $\varphi_{R}^{\mathcal{C}}\left(\bar{x}_{1}\right)$ is a conjunction of atomic formulas with predicates in $\mathcal{C}$ and $\varphi_{R}^{\mathcal{P}}\left(\bar{x}_{2}\right)$ is a conjunction of atomic formulas with predicates in $\mathcal{P}$.

Example 2.3. (example 2.2 cont.) The quality instance for John quality concerns are obtained based on (4) (Table 2.3) through the following mapping:

$$
\text { PatientValue }(p, v, t) \leftarrow \text { Measurement }(p, v, t, b), \text { Registered }(t, p)
$$

The different data sources, including the original $D$ and any at the context level, the definitions of the quality predicates in terms of elements in $\mathcal{C}$, the schema mappings and view definitions, etc., determine a collection $\mathcal{I}$ of admissible contextual instances (ACI)s for the contextual schema $\mathcal{C}$, as it is the case in virtual data integration and peer data exchange systems. 


\begin{tabular}{|c|c|c|}
\hline \multicolumn{3}{|c|}{ Table 2.3: PatientValue } \\
\hline Patient & Value & Time \\
\hline Tom Waits & 38.5 & $11: 45 / 5 / \mathrm{Sep} / 2011$ \\
Tom Waits & 38.2 & $12: 10 / 5 / \mathrm{Sep} / 2011$ \\
Tom Waits & 38.1 & $11: 50 / 6 / \mathrm{Sep} / 2011$ \\
Tom Waits & 38.0 & $12: 15 / 7 / \mathrm{Sep} / 2011$ \\
Tom Waits & $110 / 70$ & $11: 45 / 8 / \mathrm{Sep} / 2011$ \\
Lou Reed & 37.9 & $12: 10 / 5 / \mathrm{Sep} / 2011$ \\
\hline
\end{tabular}

For each ACI $I \in \mathcal{I}$, by applying the mapping such as (3), we obtain (possibly virtual) extensions $R_{\mathcal{P}}^{\prime}(\mathrm{I})$ for the predicates $R \in \mathcal{S}$. Notice that the collections of predicate extensions $R_{\mathcal{P}}^{\prime}(\mathrm{I}) \mathrm{s}$ can be seen as a quality instance $D_{\mathcal{P}}^{\prime}(\mathrm{I})$ for the original schema $\mathcal{S}$.

The quality of $R(D)$ in instance $D$ is assessed through its 'distance' to $R_{\mathcal{P}}^{\prime}(\mathrm{I})$; and the quality of $D$ in terms of an aggregated distance to $D_{\mathcal{P}}^{\prime}(I)$. By distance, we mean the numerical distance $\left|R(D) \triangle R_{\mathcal{P}}^{\prime}(I)\right|$ of the symmetric difference between the two Rinstances. And for the whole instance, e.g. the quality measure:

$$
q m_{0}(D):=\Sigma_{R \in \mathcal{S}}\left|R(D) \triangle R_{p}^{\prime}(I)\right|
$$

Example 2.4. The quality of the relation PatientValue (Table 1.1) is assessed by comparing it to the relation PatientValue' (Table 2.3). In this case $q m_{0}$ is evaluated, 1 which means that there exists one tuple in relation PatientValue' that should not be appeared in the doctor's view of data based on the quality conditions that he has asked for.

We can have several contextual instances $I \in \mathcal{I}$, thus, we can have a whole class $\mathcal{D}$ of 
instances $D_{\mathcal{P}}^{\prime}(I)$ with $I \in \mathcal{I}$. This situation is illustrated in Figure 5 .

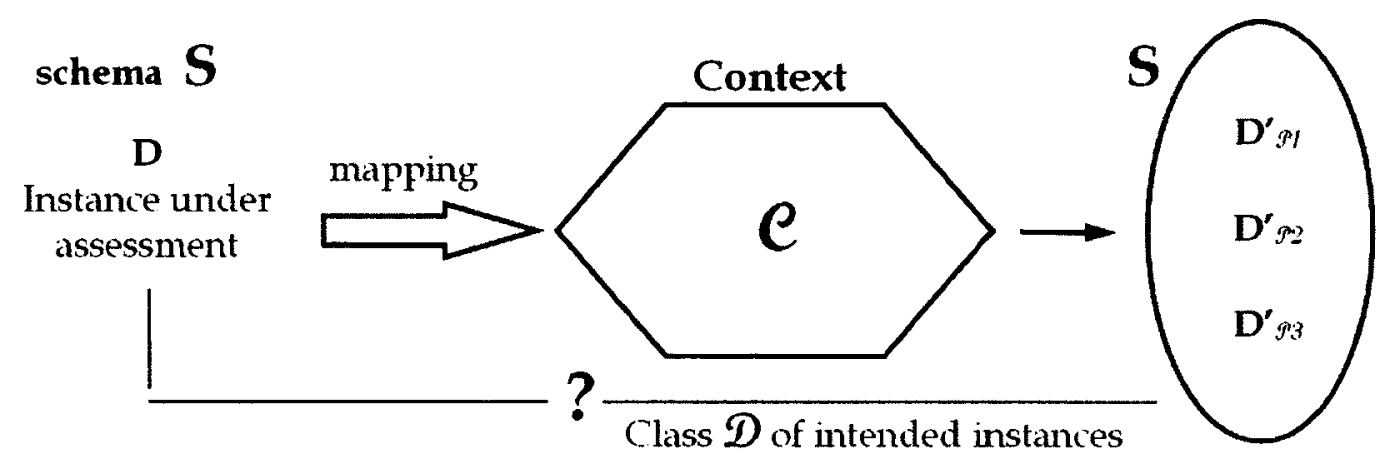

Figure 5: Using a Context for Quality Assessment

In the case of multiple 'intended' clean instances, related to a whole class $\mathcal{I}$ of ACIs, $D$ would have to be compared with a whole class of quality instances. In this case the following quality measure is introduced in [15]:

$$
q m_{1}(D):=\frac{|D|-\max \left\{\left|D_{\mathcal{P}}^{\prime}(I)\right|: I \text { is } \mathrm{LCI}\right\}}{|D|}
$$

The value of $q m_{1}$ ranges over $[0,1]$, having the value 0 when the instance $D$ is the quality data and the value 1 (or almost 1), if no tuples in $D$ satisfies the quality conditions.

\subsection{Multidimensional Databases}

In the data quality assessment framework, data under quality assessment can be viewed from different perspectives, such as geographical area, time, etc. Each perspective is represented by a dimension and expressed in different degrees of details.

Dimensions can be made part of a context by embedding in it a multidimensional database. For this purpose we can use an extension of the Hurtado-Mendelzon (HM) 


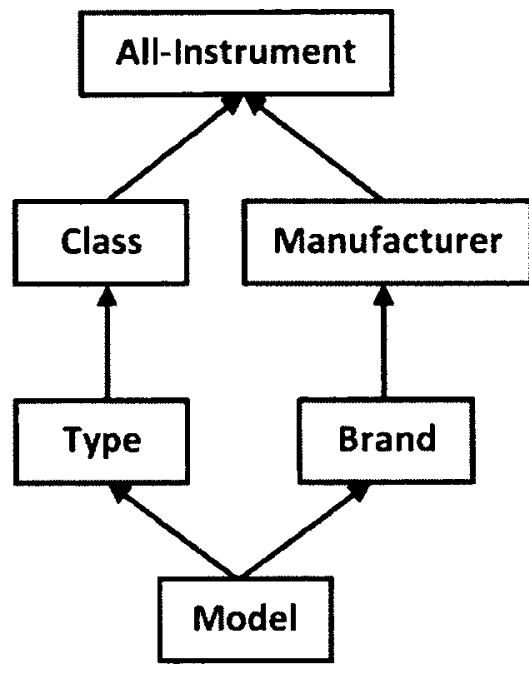

(a) Schema

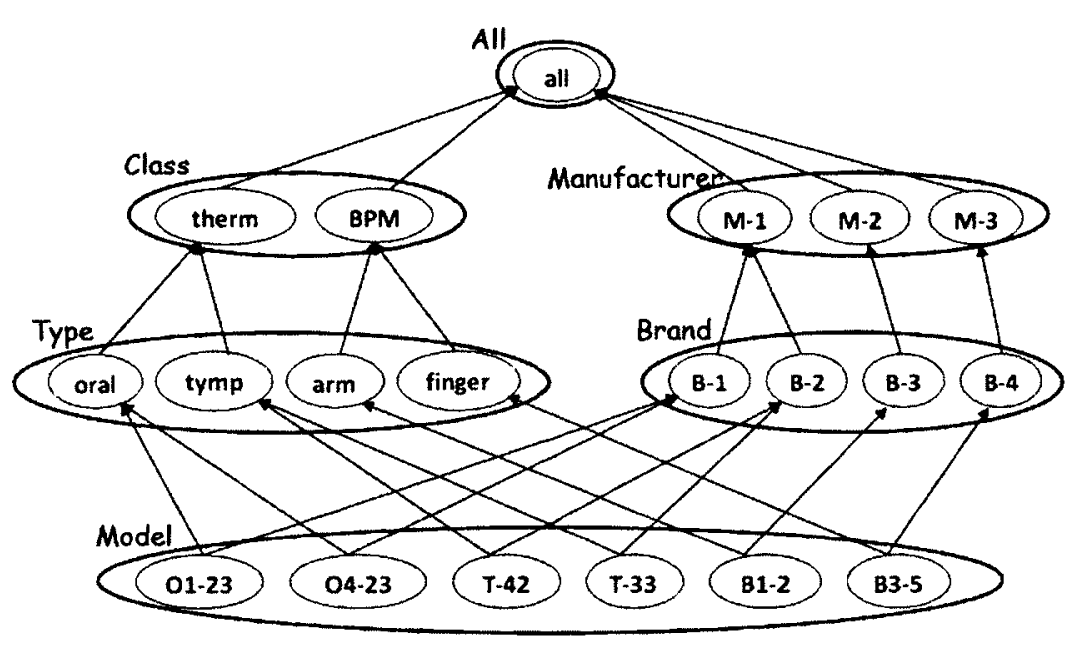

(b) Instance

Figure 6: Instrument Dimension Schema and an Instance

data model for multidimensional databases (MDDBs) [37]. In the following, we briefly described the HM model.

A contextual dimension schema $D S$ is a pair $(C A T, \nearrow)$, where $C A T$ is a set of named categories, and $\nearrow$ is a child/parent relation between categories [37]. The transitive closure of $\nearrow$ is denoted by $\nearrow^{*}$. There are no 'shortcuts' between categories. There is also a distinguished top category, denoted with All, which is reachable from all other categories. The categories without an incoming $\nearrow$ are called bottom or base categories.

An instance of a contextual $D S$ is a tuple $(M,<)$, where $M$ is a finite collection of ground atoms of the form $C(a)$ with $C \in C A T$, and the data element $a$ belongs to an underlying domain. We assume that different categories do not have elements in common. Relation $<$ stands for the partial order between elements of categories, and parallels the partial order $\nearrow$ between the corresponding categories. Category All has only one element, all. Categories are assumed to be disjoint. The transitive closure of $<$ is denoted with $<^{*}$.

Example 2.5. The dimension instance in Figure $6 \mathrm{~b}$ is defined over the Instrument dimension schema (Figure 6a), and consists of: 


$$
\begin{aligned}
M= & \{\text { Model }(\text { O1-23 }), \text { Model }(O 4-23), \text { Model }(T-42), \text { Model }(T-33), \\
& \text { Model }(B 1-2), \text { Model }(B 3-5), \text { Type }(\text { oral }), \text { Type }(\text { tymp }), \text { Type }(\text { finger }), \\
& \text { Type }(\text { arm }), \text { Brand }(B-1), B \text { rand }(B-2), \text { Brand }(B-3), \text { Brand }(B-4), \\
& \text { Class }(\text { therm }), \text { Class }(B P M), \text { Manufacturer }(M-1), \\
& \text { Manufacturer }(M-2), \text { Manufacturer }(M-3), \text { All-Instrument }(\text { all })\}, \\
<= & \{(\text { O1-23, oral }),(O 4-23, \text { oral }),(T-42, \text { tymp }),(T-33, \text { tymp }),(B 1-2, \text { arm }), \\
& (B 3-5, \text { finger }),(O 1-23, B-1),(O 4-23, B-1),(T-42, B-2),(T-33, B-2), \\
& (B 1-2, B-3),(B 3-5, B-4),(\text { oral }, \text { therm }),(\text { tymp }, \text { therm }),(\text { arm }, B P M), \\
& (\text { finger }, B P M),(B-1, M-1),(B-2, M-1),(B-3, M-2),(B-4, M-3), \\
& (B P M, \text { all }),(\text { therm }, \text { all }),(M-1, \text { all }),(M-2, \text { all }),(M-3, \text { all })\} .
\end{aligned}
$$

Relation $<^{*}$ includes all the elements in $<$ plus others, such as (O1-23, Therm).

In the HM model, a roll-up relation is defined between each two categories $C_{i}$ and $C_{j}$, and is denoted by $\mathcal{R}_{C_{i}}^{C_{j}}(D)$. Here, the roll-up relation is a set of pairs: $\{(a, b) \mid$ $C_{i}(a), C_{j}(b) \in M$ and $\left.a<^{*} b\right\}$.

A dimension $D S=(M,<)$ is said to be strict if every element of the dimension has at most one ancestor element in each of the ancestor categories, more precisely, for every different elements $a, b, c$ with $a<^{*} b$ and $a<^{*} c$, it implies $\delta(b) \neq \delta(c)$ [14].

A dimension instance of $D S$ is called homogeneous if, for every pair of categories $C_{i} \nearrow C_{j}$ and $C_{i}(a)$, there is $C_{j}(b)$ such that $a<b[14]$ [37].

\subsection{Extended Entity-Relationship Model}

An Extended Entity-Relationship Model [6] [9] (EER) is used in the conceptual representation of a data warehouse in [28] [29] (see Section 2.5.1). An entity-relationship model 
$(E / R)$ is a conceptual representation that provides a higher level of abstraction in describing the participating concepts and relations between concepts. The aim of an E/R design is to achieve a representation of data that is independent of implementation issues. The E/R diagram composed of the following elements [26]:

- Entities: Represent objects of the world being modeled (or a particular collection or class of them) and usually denoted a person, place, thing, or event of informational interest.

- Relationships: Represent real-world associations among one or more entities.

- Attributes: Represent properties of entities or relationships.

An entity relationship model is illustrated in Figure 7. The relationship Work is relating entities Hospital and Employee. Emp \# is an attribute for the entity Employee.

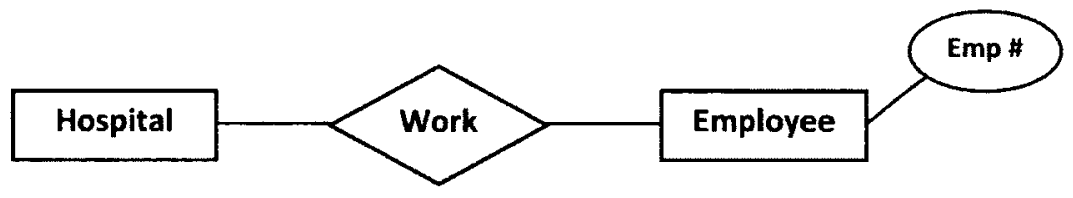

Figure 7: Entity Relationship Model

The extended entity-relationship model refers to different extensions to Chen's original entity-relationship model [26]. The extensions include an illustration of 'IS-A' link between two entities. Sub-entities (subclasses of classes) can be specified using 'IS-A'arrows connecting entities. Attributes (properties) and participation in relationships are inherited by sub-entities.

In Figure 8, an entity Nurse is a sub-entity of an entity Employee. The 'IS-A' relation between them is shown by an arrow from the entity Nurse to the entity Employee. 


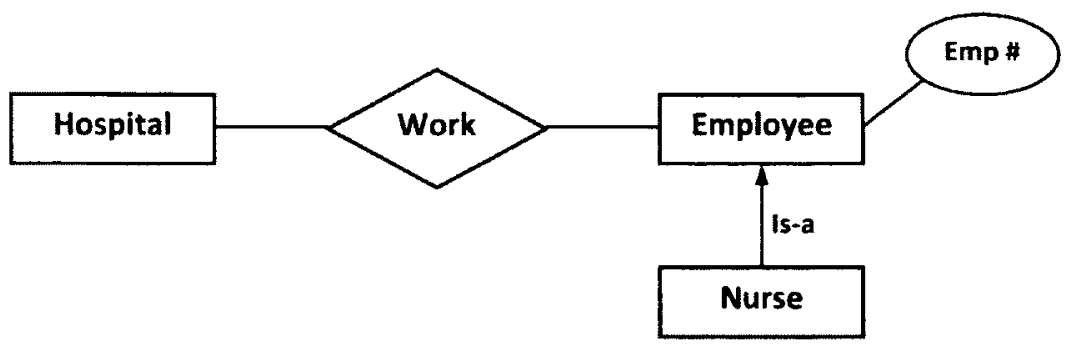

Figure 8: Extended Entity Relationship Model

\subsection{Ontologies}

An ontology is a formal, explicit specification of a shared conceptualization [31]. A conceptualization is an abstract, simplified view of the world that we wish to represent. The term 'ontology' is borrowed from philosophy, where it means a systematic account of existence.

An ontology provides a shared vocabulary for modeling a domain. The vocabulary includes types of objects and/or concepts that exist, their properties and their relations.

Ontologies are written in different languages such as OWL [46], RDF [41], RDFS [3], DL [8], etc. However, the different languages share many structural similarities such as a description of individuals (instances), classes (concepts), attributes, and relations.

Descriptions logics (DL) are a family of knowledge representation formalisms that are useful for defining, integrating, and maintaining ontologies [7]. In the following, we briefly introduce the family of DL.

\subsubsection{Description Logics}

The knowledge of an application domain is represented in a DL ontology both at the intentional level, in a TBox ' $\mathcal{T}$ ' (for terminological), and at the extensional level, in a ABox ' $\mathcal{A}$ ' (for assertions).

Basic elements are atomic concepts and atomic roles. A concept is a unary predicate gathering common properties among a collection of individuals. A role is a binary predicate 
for representing the inter-relationship between individuals. Complex descriptions can be built from elementary descriptions inductively by applying suitable concept constructors, where the set of available constructs depends on the specific description logic.

Example 2.6. Consider atomic concepts Female and Male. A concept Person is defined as a complex concept which can be either male or female. If the disjunction between the concepts is represented by ' $\sqcup$ ', the concept Person is defined in the DL TBox as follows:

$$
\text { Person } \equiv \text { Female } \sqcup \text { Male }
$$

In the spirit of having a context as a theory, we provide a DL ontological representation of the contexts as enriched with dimensions.

Ontological, DL-based representations of dimensions have been previously introduced in [28] [29], as representations of data warehouse conceptual schemas. Their representation is based on the description of DWH schemas in extended entity relationship (EER) diagrams. They provide the formal semantics for the EER representation via the $\mathcal{A L C F I}$ description logic [35]. In addition, in [28] [29], the proposed conceptual data model is used to represent complex descriptions for the structure of the aggregated entities. Accordingly, the high expressive $\mathrm{DL}, \mathcal{A} \mathcal{C} \mathcal{C} \mathcal{F}$, which includes constructs such as concept disjunction and role composition, is used to provide the semantic to the proposed EER including aggregated entities.

In Chapter 4, we will sketch a DL-based representation of our extended contextual $\mathcal{M D}$ model in one of the members of the DL-Lite family [23]. In general, DL-Lite and its extensions have a good balance of expressive power and good computational properties; and have found interesting applications in data management and semantics web [24].

In the following, we introduce the $D L$-Lite family of description logic as a language for translating the context into the DL ontology in Chapter 4. 


\section{Syntax and Semantics of the Logics in the DL-Lite Family}

DL-Lite contains object names: $a_{0}, a_{1}, \ldots$ Concepts are unary predicates and denote sets of individuals. In $D L$-Lite, concept names are represented by $A_{0}, A_{1}, \ldots$ Roles are binary predicates and denote binary relationships between individuals. In DL-Lite, role names are illustrated by $P_{0}, P_{1}, \ldots$

Roles $R$ and concepts $C$ are defined by the following grammar:

$$
\begin{array}{rll}
R::=P_{k} \mid P_{k}^{-}, & & \text {(roles) } \\
B::=\perp \mid A_{k}, & & \text { (basic concepts) } \\
C::=B|\neg C| C_{1} \sqcap C_{2}, & & \text { (complex concepts) }
\end{array}
$$

where the role $P_{k}^{-}$is an inverse of the atomic role $P_{k}$, the concept $\perp$ is a bottom concept which is always interpreted as the empty set, $\neg \mathrm{C}$ is the negation of the concept $C$.

In an extension of DL-Lite, DL-Lite ${ }^{\mathcal{N}}$, we find number restrictions of the form $\geq q R$ ( $R$ is a role). Number restrictions allow to restrict the number of role-successors, which means, the number of those objects, an object is related to via a role. If $q$ is 1 in the concepts of the form $\geq q R$, is denoted with $\exists R$, and is called an existential concept. The concepts of the form $\geq q R$ are also called basic concepts.

Example 2.7. Consider the atomic concept Person and the atomic role hasJob. The complex concept Employee is defined as: Person $\sqcap \exists h a s J o b$, where $\exists$ has Job is a basic concept constructed using a number restriction with $q=1$.

A DL-Lite Horn $_{\text {TBox }}^{\mathcal{N}}$ is a finite set of concept inclusion axioms of the form: ${ }^{1}$

$$
B_{1} \sqcap B_{2} \sqsubseteq B
$$

where all the $B_{i}$ and $B$ are basic concepts.

\footnotetext{
${ }^{1}$ Notice that in this extensions of $D L$-Lite we do not have explicit negation of concepts, as it is the case in its krom or bool extensions.
} 
The extension $D L-L i t e_{\text {Horn }}^{\mathcal{H N}}$ of $D L-L i t e_{\text {Horn }}^{\mathcal{N}}$ includes role inclusion axioms $R_{1} \sqsubseteq R_{2}$. Role inclusions are used as a basis for defining the property of transitive role of the form $\operatorname{Tra}\left(P_{k}\right)$, producing the extension DL-Lite ${ }_{\text {Horn }}^{(\mathcal{H N})^{+}}[5]$.

An occurrence of a concept on the right-hand (left-hand) side of a concept inclusion is called negative if it is in the scope of an odd (even) number of negations $\neg$; otherwise the occurrence is called positive.DL-Lite $e_{H o r n}^{(\mathcal{H N})^{+}}$allows the positive occurrences of qualified number restriction $\geq q R . C$ ( $R$ is a role and $C$ is a concept) in concept inclusions, where the number restrictions are concerned with role-fillers belonging to a certain concept.

Example 2.8. Consider the basic concept Nurse and a role Manage. If the role Manage is declared as a transitive role using the axiom Tra(Manage), the concept Supervisor is a complex concept which is defined as a nurse who manages other nurses. Concept Supervisor is defined in a DL TBox as follows:

$$
\text { Supervisor } \equiv \text { Nurse } \sqcap \exists \text { Manage.Nurse }
$$

where the concept $\exists$ Manage. Nurse is built using a qualified number restriction with $\mathrm{q}=$ 1.

The standard restriction limiting the use of transitive roles in DLs is discussed in [36]. The restriction states that only simple roles $R$ are allowed in concepts of the form $\geq q R$, for $q \geq 2$, where by a simple role in a given TBox $\mathcal{T}$ we understand a role without transitive sub-roles (including itself). In particular, if $\mathcal{T}$ contains $\operatorname{Tr} a(P)$ then $P$ and $P^{-}$are not simple, and so $\mathcal{T}$ cannot contain occurrences of concepts of the form $\geq q P$ and $\geq q P^{-}$, for $q \geq 2$.

Example 2.9. Consider the atomic role hasJob in Example 2.7. For stating the condition that each person should have at most one job, it is allowed to define an axiom such as: $\geq 2$ hasjob $\sqsubseteq \perp$ in the TBox. On the other hand, consider the role Manage in Example 
2.8. In order to express the condition that each supervisor can only manage one nurse in the form of $\geq 2$ Manage. Nurse $\sqsubseteq \perp$ is not allowed.

The formal semantics of $D L-L i t e_{H o r n}^{(H N)^{+}}$constructs is given by an interpretations $\mathcal{I}$ consisting of a non-empty set $\Delta^{\mathcal{I}}$ (the domain of the interpretation) and an interpretation function, which assigns to every atomic concept $A$ a set $A^{\mathcal{I}} \subseteq \Delta^{\mathcal{I}}$ and to every atomic role $P$ a binary relation $P^{\mathcal{I}} \subseteq \Delta^{\mathcal{I}} \times \Delta^{\mathcal{I}}$. The $D$ L-Lite ${ }_{\text {Horn }}^{(H N)^{+}}$constructs are interpreted in $\mathcal{I}$ as follows:

$$
\begin{aligned}
\left(P_{k}^{-}\right)^{\mathcal{I}} & =\left\{(y, x) \in \Delta^{\mathcal{I}} \times \Delta^{\mathcal{I}} \mid(x, y) \in P_{k}^{\mathcal{I}}\right\}, & \text { (inverse role) } \\
\perp^{\mathcal{I}} & =\emptyset, & \text { (the empty set) } \\
(\geq q R)^{\mathcal{I}} & =\left\{x \in \Delta^{\mathcal{I}}||\left\{y \in \Delta^{\mathcal{I}} \mid(x, y) \in R^{\mathcal{I}}\right\} \mid \geq q\right\}, & \text { (number restriction) } \\
(\neg C)^{\mathcal{I}} & =\Delta^{\mathcal{I}} \backslash C^{\mathcal{I}}, & \text { (not in } C \text { ) } \\
\left(C_{1} \sqcap C_{2}\right)^{\mathcal{I}} & =C_{1}^{\mathcal{I}} \cap C_{2}^{\mathcal{I}}, & \text { (both in } \left.C_{1} \text { and in } C_{2}\right)
\end{aligned}
$$

An interpretation $\mathcal{I}$ satisfies a concept inclusion $B_{1} \sqsubseteq B_{2}$ if $B_{1}^{\mathcal{I}} \subseteq B_{2}^{\mathcal{I}}$; and it satisfies a role inclusion $R_{1} \sqsubseteq R_{2}$ if $R_{1}^{\mathcal{I}} \subseteq R_{2}^{\mathcal{I}}$.

The role transitivity constraints, $\operatorname{Tr} a\left(P_{k}\right)$, states that the role $P_{k}$ must be interpreted as a transitive role:

$$
\mathcal{I} \models \operatorname{Tr} a\left(P_{k}\right) \quad \text { iff } \quad(x, y) \in P_{k}^{\mathcal{I}} \text { and }(y, z) \in P_{k}^{\mathcal{I}} \text { imply }(x, z) \in P_{k}^{\mathcal{I}} \text {, for all } x, y, z \in \Delta^{\mathcal{I}} \text {. }
$$

The qualified number restriction extension is interpreted as:

$$
(\geq q R . C)^{\mathcal{I}}=\left\{x \in \Delta^{\mathcal{I}}|\quad|\left\{y \in C^{\mathcal{I}} \mid(x, y) \in R^{\mathcal{I}}\right\} \mid \geq q\right\}
$$

A model of a DL ontology is an interpretation $\mathcal{I}$ which is a model of all assertions in the DL ontology. A DL ontology is satisfiable if it has at least one model.

Example 2.10. Consider the concepts and roles in Example 2.8. Let an interpretation $\mathcal{I}=\left\langle\Delta^{\mathcal{I}}, .^{\mathcal{I}}\right\rangle$ has a domain as follows: 


$$
\Delta^{\mathcal{I}}=\{\text { Kate, Mary, John }\}
$$

The role Manage is declared as a transitive role by an axiom $\operatorname{Tra}($ Manage), the concepts and roles of Example 2.8 are interpreted by an interpretation function.$^{\mathcal{I}}$ as follows:

$$
\begin{aligned}
\text { Nurse }^{\mathcal{I}} & =\{\text { Mary }, \text { Kate }\} \\
\text { Manage }^{\mathcal{I}} & =\{(\text { Mary }, \text { kate }),(\text { John }, \text { Mary }),(\text { John }, \text { kate })\}
\end{aligned}
$$

The DL ontology consisting of the assertions in Example 2.8 is satisfiable, since there is an interpretation such as $\mathcal{I}$ which satisfies all of its assertions. In the other words, $\mathcal{I}$ is the model of the ontology which contains the assertions in Example 2.8.

\section{Linking Data to DL-Lite Ontologies}

In an ontology-based data access (OBDA) system, the TBox of the ontology provides a shared, uniform, abstract view of the intensional level of the application domain [25]. The extensional data are located in the data sources which have independent implementations from the conceptual layer.

Linking data in the existing data sources to a DL-Lite ontology is done through a mapping mechanism [50]. The idea of the representation of the mappings is that by evaluating a query over the data sources, facts for constituting the $\mathrm{ABox} \mathcal{A}$ assertions are retrieved. This idea is illustrated in Figure 9.

As discussed before, the ontology is a virtual representation of a domain of an application, and the instances of the concepts and roles in the ontology are the abstract and virtual representation of some real data stored in the existing data sources. Therefore, the problem arises of establishing sound mechanisms for linking the existing data in the data sources to the instances of the concepts and the roles in the ontology.

A solution to the problem of linking the existing data in the data sources to the instances 


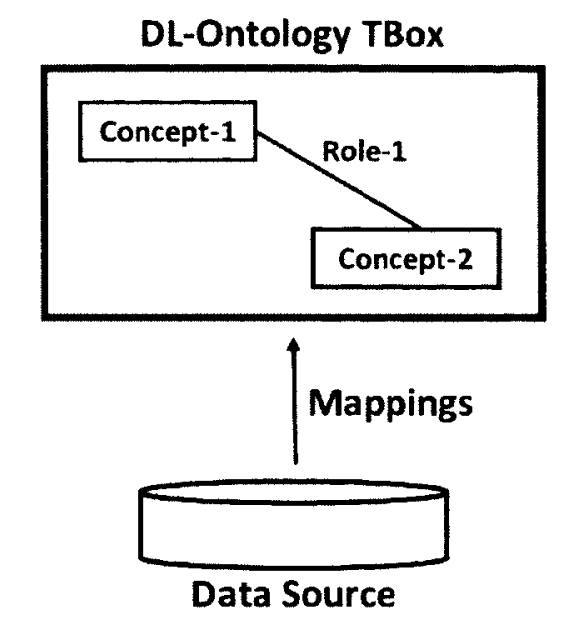

Figure 9: Linking Data to Ontologies

of the concepts and the roles in the ontology is proposed in [50]. In order to explain the solution, first some definitions and their semantics are reviewed. A set $\Gamma_{V}$ is the set of data value constants (real values in data sources), and a set $\Gamma_{O}$ is the set of object terms (abstracts elements (pair of elements) of concepts and roles) which are built starting from $\Gamma_{V}$ and a set $\Lambda$ of function symbols of any arity (possibly 0 ), as follows: If $f \in \Lambda$, the arity of $f$ is $n$, and $d_{1}, \ldots, d_{n} \in \Gamma_{V}$, then $f\left(d_{1}, \ldots, d_{n}\right)$ is a term in $\Gamma_{O}$, called object term.

The mapping assertions associate a conjunctive query over atomic concepts, domains, roles and attributes with a first-order query of the appropriate arity over the data source. The idea is that by evaluating such a query over the data source, the facts for building the ABox assertions are retrieved.

Example 2.11. Consider a DL-Lite TBox in which Person and Job are atomic concept names and hasJob is an atomic role name, and a relational database contains a binary relation occupation(person, job). We want to model the situation where $p \in$ occupation corresponds to a person whose name is $p$. Similarly, we want to model the fact that $j \in$ occupation corresponds to the name of the job that the person has. The following is the set 
of mapping assertions modeling the above situation.

$$
\begin{array}{lll}
\forall p \forall j(\operatorname{occupation}(p, j) & \rightarrow \operatorname{Person}\left(f_{\text {person }}(p)\right), \\
\forall p \forall j(\operatorname{occupation}(p, j) & \rightarrow & J o b\left(f_{\text {job }}(j)\right), \\
\forall p \forall j(\operatorname{occupation}(p, j) & \rightarrow & \operatorname{hasJob}\left(f_{\text {person }}(p), f_{\text {job }}(j)\right) .
\end{array}
$$

Above, $p$ and $j$ are variable symbols; $f_{\text {person }}$ and $f_{j o b}$ are function symbols; and $f_{\text {person }}(p)$ and $f_{j o b}(j)$ are variable object terms.

\section{Inference Services in $D L$-Lite}

In this thesis, we concentrate on three standard reasoning tasks for DL-Lite family of description logics: satisfiability, instance checking, and query answering.

Satisfiability: Let $\mathcal{O}$ be a $D L$-Lite ontology. The satisfiability problem is to check whether there is a model of $\mathcal{O}$.

Concept Instance Checking: Let $\mathcal{O}$ be a $D L$-Lite ontology, $C$ be a concept and $a$ be an individual. The individual $a$ is an instance of $C$ with respect to $\mathcal{O}$ if and only if, $a^{\mathcal{I}} \in C^{\mathcal{I}}$ in all of the models $\mathcal{I}$ of $\mathcal{O}$.

Role Instance Checking: Let $\mathcal{O}$ be a $D L$-Lite ontology, $R$ be a role, and $a, b$ be individuals. The pair of individuals $(a, b)$ is an instance of $R$ with respect to $\mathcal{O}$ if and only if $\left(a^{\mathcal{I}}, b^{\mathcal{I}}\right) \in R^{\mathcal{I}}$ in all of the models $\mathcal{I}$ of $\mathcal{O}$.

Query Answering: Let $\mathcal{O}$ be a $D L$-Lite ontology and $\mathcal{Q}$ be a query over $\mathcal{O}$. The query answering over the $\mathcal{O}$ is to compute the set of certain answers to $\mathcal{Q}$ over $\mathcal{O}$. For the ontology $\mathcal{O}$, we say that a tuple $a$ of object names from $\mathcal{A}$ is a certain answer to $\mathcal{Q}(x)$ with respect to $\mathcal{O}$, if $\mathcal{I} \models \mathcal{Q}(a)$ whenever $\mathcal{I} \models \mathcal{O}$.

\section{Complexity of Reasoning in DL-Lite}

Complexity measures depend on those parameters of the problem that are considered as 
the input and those that are considered to be fixed. For verifying the satisfiability of a DL-Lite ontology and the concept and role instance checking of a DL-Lite ontology, the parameters to consider are the size of the TBox $\mathcal{T}$, and the size of the $\mathrm{ABox} \mathcal{A}$. The size of the TBox $\mathcal{T},|\mathcal{T}|$, is defined as the number of symbols in $\mathcal{T}$, and the size of the ABox $\mathcal{A}$, $|\mathcal{A}|$, is defined as the number of symbols in $\mathcal{A}$. For query answering, one more parameter to consider is the size of the query. However, we assume that the size of the query is insignificant in comparison to the size of the TBox and the size of the ABox. Thus the size of the query is not considered as part of the input.

If the whole DL-ontology is regarded as an input, then our concern is combined complexity. If only the $\mathrm{ABox} \mathcal{A}$ is regarded as an input, while the TBox $\mathcal{T}$ (and the query) is assumed to be fixed, then we deal with data complexity [55]. In ontology-based data access, data complexity is the point of interest since the size of the TBox is fixed and the size of the query is negligible compared to the size of the ABox [22].

For combined complexity, checking the satisfiability of the ontology that is expressed in $D L-L i t e_{H o r n}^{(\mathcal{H N})^{+}}$is P-complete. Satisfiability, instance checking and query answering of the $D L-$ Lite $_{\text {Horn }}^{(\mathcal{H N})^{+}}$ontology is NLogSpace-complete for data complexity [5].

Adding the role transitivity constraint, $\operatorname{Tra}(R)$ to $D L$-Lite $e_{\text {horn }}^{(H N)}$, producing $D L$ Lite $_{\text {horn }}^{(H N)^{+}}$, results in the increase of the data complexity for all reasoning problems to NLogSpace-complete, although it doesn't have any effect on checking the satisfiability for combined complexity.

\section{Query Answering in Description Logic}

In order to query a DL ontology such as $\mathcal{O}$, the first assumption should be made on the satisfiability of the DL ontology. In the case where the DL ontology is unsatisfiable, the answer to a query $\mathcal{Q}$ is defined as a finite set of tuples $\operatorname{AllTup}(\mathcal{Q}, \mathcal{O})$.

As mentioned before, we restrict ourselves to conjunctive queries and union of conjunctive queries. Conjunctive queries are the good suit for expressing complex queries and 
remain decidable in the presence of incomplete information.

A conjunctive query over a $D L$ ontology $\mathcal{O}$ is a conjunctive query whose atoms are of the form $A_{k}(t)$ and $P_{k}\left(t_{1}, t_{2}\right)$, where $A_{k}$ is a concept name, $P_{k}$ a role name, and $t, t_{1}, t_{2}$ are terms taken from the list of variables $y_{0}, y_{1}, \ldots$ and the list of object names $a_{0}, a_{1}, \ldots$

Example 2.12. A conjunctive query asks for the type of the hospital $H_{1}$ :

$$
\exists h\left(\text { Hospital }(h) \wedge \text { HospitalType }(h, t) \wedge h=H_{1}\right) \rightarrow \mathcal{Q}(t)
$$

Here, $\mathcal{Q}$ is a predicate that collects the query answers.

The set of answers to a conjunctive query $\exists y \operatorname{con} j(x, y) \rightarrow \mathcal{Q}(x)$, where $x$ is a distinguished variable, $\mathrm{y}$ is an existentially quantified variable, and $\operatorname{conj}(x, y)$ is a conjunction of atoms (concepts and roles) is interpreted in $\mathcal{I}$ as the set $\mathcal{Q}^{\mathcal{I}}$ of tuples $e \in \Delta^{\mathcal{I}} \times \ldots \times \Delta^{\mathcal{I}}$ such that, when we assign $e$ to the variables $x$, the formula $\operatorname{con} j(x, y)$ evaluates to true in $\mathcal{I}$.

The set of certain answers to the conjunctive query $\mathcal{Q}$ over the ontology $\mathcal{O}$, denoted $\operatorname{cert}(\mathcal{Q}, \mathcal{O})$ are the tuples $e$ of constants of $\mathcal{A}$ such that $e \in \mathcal{Q}^{\mathcal{I}}$, for every model $\mathcal{I}$ of $\mathcal{O}$.

\section{Description Logic Reasoners}

Different DL reasoners with various capabilities have been developed for reasoning over the ontologies written in DL such as: HermiT [53], RACERPro [34], KAON2 [1], Pellet [54], Quest [2], etc.

Hermit is based on a novel 'hypertableau' calculus [48] and can determine whether or not the ontology is consistent, identify subsumption relationships between classes, etc. RacerPro is a knowledge representation system that implements a tableau calculus for a very expressive description logic $\mathcal{S H I Q}$ [30]. In addition, RacerPro can process OWL Lite and OWL DL ontologies.

KAON2 is a free Java reasoner for an expressive DL $(\mathcal{S H I Q})$ which implements a 
resolution-based decision procedure for the DL ontology reasoning services. Pellet is an open-source Java based OWL DL reasoner. It is based on tableaux algorithms developed for expressive description logics and supports reasoning with the full expressivity of OWLDL.

The satisfiability of the $D L-L i t e_{h o r n}^{(\mathcal{H} N)^{+}}$ontology can be verified by the reasoners such as Pellet, KAON2, HermiT and RacerPro.

In Chapter 4, we introduce axioms for translating an extended $\mathcal{M D}$ model into a $\mathrm{DL}$ ontology. In Chapter 6, we show an experiment on the satisfiability of an ontological representation of the extended $\mathcal{M D}$. We verified its satisfiability with the Pellet reasoner. Furthermore, we show how the quality instances are obtained from the resulting ontology. 


\section{Chapter 3}

\section{Extending the Contextual Framework with Dimensions}

In the data quality assessment framework discussed in Section 2.2, an important element that was not included is the one of data dimension. Enhancing the context with the dimensions is necessary because data for data quality analysis are almost always of a dimensional or hierarchical nature. Dimensions provide different perspectives or points of view from which data can be seen and analyzed. In this scenario, we may have to go outside a contextual table, and navigate within the context, searching for the necessary data.

Multidimensional databases (MDDBs) were introduced in Section 2.3. In this chapter, we extend to the $\mathcal{M D}$ model, as needed in multidimensional contexts.

\subsection{Facts Associating Contextual Dimensions}

The data associated to the contextual dimensions are points in a multidimensional space. A fact is a data entity which is a focus of interest. In most of the multidimensional data models, facts are implicitly defined by combinations of dimensions' values. As is common in DWHs and MDDBs, in general, we can have fact tables associated to the base categories.

However, using a multidimensional model and a multidimensional database within a 
context serves different purposes from the one that is traditionally found in data warehousing, OLAP, or data analytics. We can, for example, extend the $\mathcal{M D}$ model with additional tables associated to the different levels of the hierarchies, i.e. to categories other than base categories (to which the fact tables are usually associated).

In the following, we introduce two kinds of contextual relations as extensions to the $\mathcal{M D}$ model.

\subsubsection{Categorical Relations}

Given a $\mathcal{M D}$ database, a categorical relation $(\mathrm{CR})$ is a database relation in which some of its attributes are category names (or nicknames for them). In such a case, the attribute and the category share the underlying domain. Categories in CRs may not be the base category, but belong to higher levels of the hierarchy.

In order to establish the connection between a categorical relation $R$, and the corresponding categories, we need schema mappings. More precisely, if the $i$ th position of $R$ corresponds to the category $C$ of the dimension $D$, then there is a mapping as follows:

$$
\forall x_{1} \ldots x_{n}\left(R\left(x_{1}, \ldots, x_{i}, \ldots, x_{n}\right) \rightarrow C\left(x_{i}\right)\right)
$$

Actually, this formula acts as a sort of referential constraint.

Example 3.1. The categorical relation in Table 3.1 (Figure 10) shows the values of the medical tests that are performed by different instruments on a patient, Tom at a specific point of time. It contains the attribute Hour, that corresponds to category Hour in the Time dimension schema in Figure 10. In this case the category is a base category.

The mapping between the attribute Hour of the categorical relation TomResults and category Hour of Time dimension is expressed, based on (5), as:

$$
\forall p \forall v \forall h \forall c(\text { TomResults }(p, v, h, c) \rightarrow H o u r(h))
$$




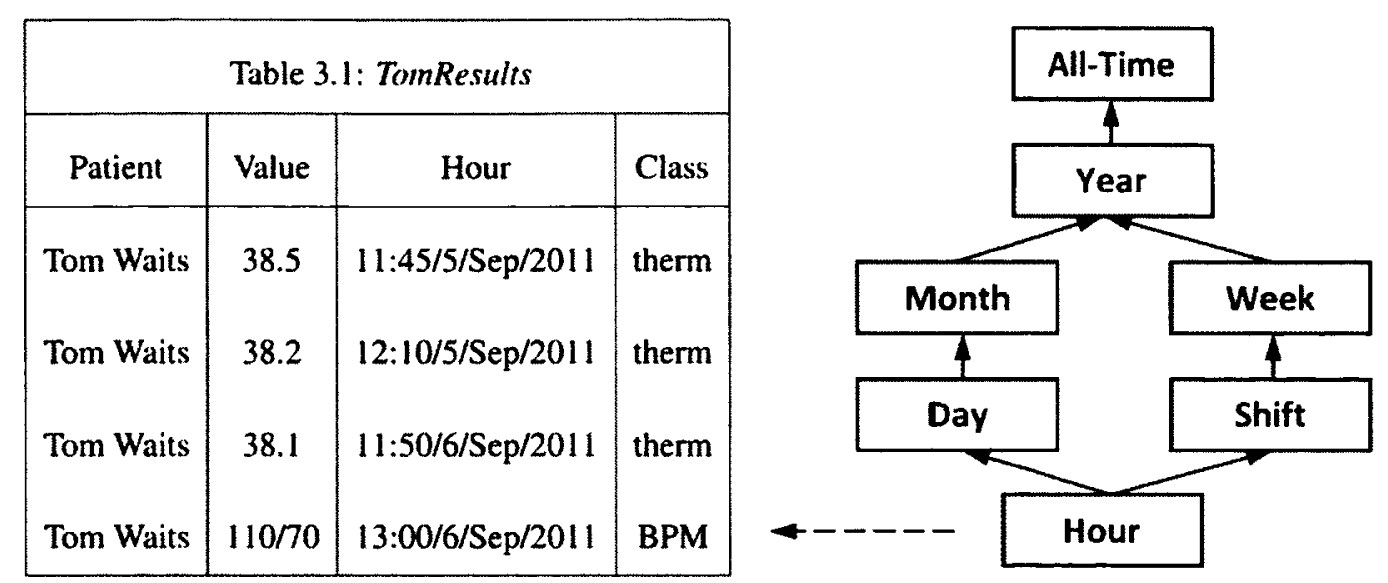

Figure 10: TomResults to Time Mapping

Example 3.2. Consider the relation UnitManuf(Table 1.6) in Figure 4. The relation UnitManuf is an example of a CR which contains the attribute Care-Unit, that corresponds to a category Care-Unit in the Location dimension schema. Accordingly, we have the mapping:

$$
\forall c \forall m \forall o(\operatorname{UnitManuf}(c, m, o) \rightarrow \text { Care-Unit }(c))
$$

In this case the category is not a base category. Notice that this CR contains categories from two different dimensions.

\subsubsection{Attributive Relations}

The other extension to the $\mathcal{M D}$ model is the introduction of attributive relations (AR). An attributive relation is connected to a single category of a single dimension schema. Each AR provides, through its attributes, a description for the elements of the category in terms of mappings between the relational and dimensional schemas. We need a mapping of the form:

$$
\forall x_{1} \ldots x_{n}\left(R\left(x_{1}, \ldots, x_{i}, \ldots, x_{n}\right) \rightarrow C\left(x_{i}\right)\right)
$$

Each member of a category $C$ in a contextual dimension $D$ can accept at most one value 
for each of the attributes in an attributive relation AR. This condition is represented as:

$$
\forall x \forall x_{1} \forall x_{2}\left(C(x) \wedge R\left(x, x_{1}\right) \wedge R\left(x, x_{2}\right) \rightarrow x_{1}=x_{2}\right)
$$

ARs may provide descriptions for all of the elements of the category. Thus, in addition to (7), the following mapping may also be introduced for ARs:

$$
\forall x \exists y_{1} \ldots y_{n}\left(C(x) \rightarrow R\left(y_{1}, \ldots, x, \ldots, y_{n}\right)\right)
$$

Notice that from the formal point of view, a one-category categorical relation, i.e. with a single categorical attribute, does not differ from an attributive relation. However, the latter kind is always associated to a single category, whose elements are described through the other attributes.

As opposed to CRs, an AR is not meant to support dimensional navigation via the categorical attributes. Navigation is a process that could be realized with the machinery developed in [43] [44], that is an extension of relational algebra, denoted as Contextual Relational Algebra (CRA), for querying data at different levels of details.

Example 3.3. Consider the dimension Instrument in Figure 6a. Let relation ManufacturerDescription (Table 3.2) is an attributive relation which provides descriptions about the type of the instruments' manufacturers and the kind of industry manufacturer belongs to.

\begin{tabular}{|c|c|c|}
\hline \multicolumn{2}{|c|}{ Table 3.2: ManufacturerDescription } \\
\hline Manufacture & Type & Industry \\
\hline $\mathbf{M}_{1}$ & Public & Electronics \\
$\mathbf{M}_{2}$ & Private & Electronics \\
$\mathbf{M}_{3}$ & Public & Conglomerate \\
\hline
\end{tabular}


The following mappings are defined between the attribute Manufacturer of ManufacturerDescription and the Manufacturer category of the Instrument dimension:

$$
\begin{aligned}
\forall m \forall t \forall i(\text { ManufacturerDescription }(m, t, i) & \rightarrow \text { Manufacturer }(m)) . \\
\forall m \exists t \exists i(\text { Manufacturer }(m) & \rightarrow \text { ManufacturerDescription }(m, t, i)) .
\end{aligned}
$$

Each manufacturer can be at most one type, which is expressed as:

$$
\begin{aligned}
\forall m \forall t_{1} \forall t_{2} \forall i \quad & \text { ManufacturerDescription }\left(m, t_{1}, i\right) \\
& \wedge \\
\text { ManufacturerDescription }\left(m, t_{2}, i\right) & \left.\rightarrow t_{1}=t_{2}\right)
\end{aligned}
$$

Example 3.4. Let HospitalDescription (Table 1.7) in Figure 4 be an example of an AR which provides descriptions for the elements of the Hospital category of dimension Location. Accordingly, we have the following mapping:

$$
\forall h \forall t \forall s(\text { HospitalDescription }(h, t, s) \rightarrow \text { Hospital }(h)) .
$$

Since the relation HospitalDescription provides descriptions for all of the elements of the Hospital category, the following constraint should also be imposed:

$$
\forall h \exists t \exists s(\text { Hospital }(h) \rightarrow \text { HospitalDescription }(h, t, s)) .
$$

Each hospital can be at most one type, which is expressed as:

$\forall h \forall t_{1} \forall t_{2} \forall s\left(\right.$ HospitalDescription $\left(h, t_{1}, s\right) \wedge$ HospitalDescription $\left.\left(h, t_{2}, s\right) \rightarrow t_{1}=t_{2}\right)$

\subsection{Semantic Constraints}

In this section, we introduce semantic constraints over the categorical and the attributive relations. 


\subsubsection{Interdimensional Constraints}

A categorical relation represents data which are the focus of interest from different perspectives and in different granularities through the combination of dimensions' values.

In categorical relations, generally, not all of the combination of the contextual dimensions' values are point of interest. More precisely, some combinations of contextual dimensions' values in categorical relations are not allowed. Interdimensional constraints prohibit combinations of values from several dimensions in categorical relations.

Example 3.5. Assume we want to express that a medical test on a patient can not be performed with two different instruments from the same class at the same time. For example, it is not possible to record two temperature values for a patient at the same time by different thermometers.

Consider the categorical relation PatientTestsDetails(Patient, Value, Hour, Model) in Table 3.3. Relation PatientTestsDetails is linked to the Time dimension (Figure 10) through its attribute Hour, and also it is connected to the Instrument dimension (Figure 6) through its last attribute Model. It contains information about the different medical tests that are performed on patients at the specific time.

The previously mentioned assumption can be expressed as an interdimensional constraint in the form of a denial constraint as:

$\neg \exists p v h m_{1} m_{2} c_{1} \quad\left(\right.$ PatientTestsDetails $\left(p, v, h, m_{1}\right) \wedge$ PatientTestsDetails $\left(p, v, h, m_{2}\right)$ $\wedge$ Instrument $\left(m_{1}, c_{1}\right) \wedge$ Instrument $\left.\left(m_{2}, c_{1}\right) \wedge m_{1} \neq m_{2}\right)$

Such a constraint involves categories from two different dimensions. 


\begin{tabular}{|c|c|c|c|}
\hline \multicolumn{4}{|c|}{ Table 3.3: PatientTestsDetails } \\
\hline Patient & Value & Hour & Model \\
\hline Tom Waits & 38.5 & $11: 45 / 5 / \mathrm{Sep} / 2011$ & O1-23 \\
Tom Waits & 38.2 & $12: 10 / 5 / \mathrm{Sep} / 2011$ & O1-23 \\
Tom Waits & 38.1 & $11: 50 / 6 / \mathrm{Sep} / 2011$ & O1-23 \\
Tom Waits & 38.0 & $12: 15 / 7 / \mathrm{Sep} / 2011$ & O4-23 \\
Tom Waits & $110 / 70$ & $11: 45 / 8 / \mathrm{Sep} / 2011$ & B3-5 \\
Lou Reed & 37.9 & $12: 10 / 5 / \mathrm{Sep} / 2011$ & T-42 \\
\hline
\end{tabular}

Note that the PatientTestsDetails can still contains medical tests results from the same class of instruments at different times.

Example 3.6. Suppose we want to specify the requirement that no single measurement can be taken by more than one nurse. For this, we can use relationPatientValue (Table 1.1) which that can be seen as a CR linked to the Time dimension through its last attribute; and also PatienWard (Table 1.4), a CR linked to both the Location and Time dimensions. We also need to appeal to the Nurse category of the Location category. With all these elements, the above requirement can be expressed as an interdimensional constraint:

$$
\begin{aligned}
\neg \exists p v t d w n_{1} n_{2} & (\text { PatientValue }(p, v, t) \wedge \text { PatientWard }(p, d, w) \wedge T(t, d) \wedge \\
& \left.L\left(n_{1}, w\right) \wedge L\left(n_{2}, w\right) \wedge n_{1} \neq n_{2}\right) .
\end{aligned}
$$

$T$ and $L$ are binding predicates for the partial orders $(<)$ between elements of categories Time and Day in the Time dimension, and Nurse and Ward in the Location dimension, 
respectively. This constraint takes the form of a denial constraint, that prohibits certain combinations of atoms.

\subsubsection{Intradimensional Constraints}

Contextual dimensions consist of sets of categories that are ordered in different levels of a hierarchy. Each category represents the group of members of its child categories.

Attributive relations are associated to a single category which is also connected to its parent category through the partial order relation in a single dimension schema.

Generally, not all of the descriptive values of attributive relations can be associated to the elements of a category in a dimension. Intradimensional constraints restrict certain combinations of descriptive values (in attributive relations) associated to elements in different categories that are $<^{*}$-connected.

Example 3.7. (example 1.1 cont.) Consider the contextual dimension Location in Figure $3 \mathrm{~b}$, in which each member of a Hospital category is a parent of possibly several members of the Care-Unit category. Each member of a Care-Unit category is a parent of several members of the Ward category.

In hospital $H$, patients may have some visitors during visiting hours. However, not all of the care units in the hospital let patients have visitors, even during the visiting hours. For example, patients in the post-anesthetic care unit are not allowed to have visitors.

Permission for having visitors in each care unit is expressed by an attribute VisitorAllowedU in an attributive relation CareUnitDescription (Table 3.4).

In some wards such as $W_{1}$, based on the patients' conditions, having visitors during the visiting hours is prohibited. The permission for having visitors in each ward during visiting hours is represented by an attribute VisitorAllowedW in attributive relation WardDescription (Table 3.5). 


\begin{tabular}{|c|c|}
\hline \multicolumn{2}{|c|}{ Table 3.4: CareUnitDescriptio } \\
\hline Care-Unit & VisitorAllowedU \\
\hline standard & YES \\
\hline intensive & NO \\
\hline terminal & YES \\
\hline
\end{tabular}

\begin{tabular}{|c|c|}
\hline \multicolumn{2}{|c|}{ Table 3.5: WardDescription } \\
\hline Ward & VisitorAllowedW \\
\hline$W_{1}$ & NO \\
$W_{2}$ & YES \\
$W_{3}$ & YES \\
\hline
\end{tabular}

If having visitors in a care unit is prohibited, the patients in its different wards are not allowed to have any visitors.

The relations CareUnitDescription(Care-Unit, VisitorAllowedU) and WardDescription(Ward, VisitorAllowedW) are two attributive relations that are connected to the Care-Unit and Ward categories, respectively. If $L$ stands for the partial order relation between the elements of the Location dimension, the previously mentioned requirement can be expressed as an intradimensional constraint in the form of a denial constraint as:

$$
\begin{array}{cc}
\neg \exists u v u w v w & (\text { CareUnitDescription }(u, v u) \wedge \operatorname{WardDescription}(w, v w) \wedge \\
& \left.L(w, u) \wedge v u={ }^{\prime} \mathrm{NO}^{\prime} \wedge v u \neq v w\right)
\end{array}
$$

Example 3.8. Relation OperationYear in Table 3.6 contains information about the operations which are performed in a year, and relation OperationDay (Table 3.7) represents the information about operations which are performed in a day. We expect that if there is an operation in a year, it must appear on a particular day of (associated to) that year.

\begin{tabular}{|c|c|}
\hline \multicolumn{2}{|c|}{ Table 3.6: OperationYear } \\
\hline Year & OperationID \\
\hline 2011 & 1263121 \\
2010 & S186437 \\
\hline
\end{tabular}

\begin{tabular}{|c|c|}
\hline Table 3.7: & OperationDay \\
\hline Day & OperationID \\
\hline 4/Aug/2011 & I263121 \\
5/Feb/2010 & $\mathrm{S} 186437$ \\
\hline
\end{tabular}


Consider dimension Time in Figure 10, also assume that the operation IDs are unique. If $T$ stands for the partial order relation between the elements of the Time dimension, an intradimensional constraint for representing the above assumption is expressed by:

$$
\forall o p \forall y \exists d \quad(\text { OperationYear }(y, o p) \rightarrow T(d, y) \wedge \text { OperationDay }(d, o p))
$$

Notice that interdimensional constraints and intradimensional constraints may have interesting logical interactions with the well-known semantic constraints of the HM models, e.g. about homogeneity (every element of a category rolls up to an element of a parent category) and strictness (rolls up to at most one) [37]. 


\section{Chapter 4}

\section{Ontological Representation of Contextual Dimensions}

In this chapter, with the motivation of having a full-fledged ontology as a contextual framework for data quality assessment [16], we show how the dimensions (and their extensions) were introduced in Chapters 2 and 3 can be expressed as a part of an ontology in a description logic (DL). We do this by using a DL of the DL-Lite family, more specifically, DL-Lite ${ }_{\text {Horn }}^{(\mathcal{H} N)^{+}}$(see Section 2.5.1 for details).

\subsection{Translating a Contextual Dimension Schema into a DL TBox}

In order to represent a contextual dimension schema (DS) as a DL-ontology, each category and the domain of an attribute are expressed by one atomic concept name.

Example 4.1. Consider the Location dimension schema in Figure 3a. The categories (category names) and attribute domains in attributive relations are represented as concepts in the TBox $\mathcal{T}:$ Nurse, Ward, Care-Unit, ..., String, etc.

In addition to these atomic concepts, $D L$-Lite provides the empty concept $(\perp)$. The empty concept allows us to express the disjointness of categories. 
In a dimension $D$, categories are assumed to be disjoint, that means each two different categories of the contextual dimension do not have any elements in common. In other words, the conjunction of each two concepts, while each stands for a category in the contextual dimension, should be empty. The disjointness between each two categories, such as $C_{i}$ and $C_{j}$ in the contextual dimension schema, is expressed in DL-Lite as:

$$
C_{i} \sqcap C_{j} \sqsubseteq \perp
$$

Example 4.2. In the contextual dimension Location in Figure 3, categories are assumed to be disjoint. The disjointness between categories is captured by adding axioms to the TBox $\mathcal{T}$, among others:

$$
\begin{array}{rlr}
\text { Nurse } \sqcap \text { Ward } & \sqsubseteq \perp, & \text { Ward } \sqcap \text { Care-Unit } \sqsubseteq \perp, \\
\text { Nurse } \sqcap \text { Care-Unit } & \sqsubseteq \perp, & \text { Ward } \sqcap \text { Hospital } \sqsubseteq \perp, \\
\text { Nurse } \sqcap \text { Hospital } & \sqsubseteq \perp, & \text { Ward } \sqcap \text { City } \sqsubseteq \perp, \\
\text { Nurse } \sqcap \text { City } & \sqsubseteq \perp, & \text { Ward } \sqcap \text { Country } \sqsubseteq \perp, \\
\text { Nurse } \sqcap \text { Country } \sqsubseteq \perp, & \text { Care-Unit } \sqcap \text { Hospital } \sqsubseteq \perp .
\end{array}
$$

DL-Lite offers inverse roles. In addition, if $R$ stands for a role, in an extension of $D L$-Lite, $D L$-Lite ${ }^{\mathcal{N}}$, we find concepts defined by number restrictions of the form $\geq q R$ (when $q$ is 1 , this denoted with $\exists R$ ). Number restrictions allow us to restrict the number of role-successors, which means, the number of those objects an object is related to via a role.

Each attribute of an attributive relation $A R$, associating elements of the category $C_{i}$ in a dimension $D$ with a range $V$ ( $V$ is considered as a datatype such as string, integer, etc.), is represented in the TBox as a role, say $A R_{i}$, that is functional and satisfies the following conditions: 


$$
\exists A R_{i}^{-} \sqsubseteq C_{i}, \quad \exists A R_{i} \sqsubseteq V, \quad \geq 2 A R_{i} \sqsubseteq \perp
$$

The first axiom states that the domain of the role $A R_{i}$ is category $C_{i}$, and second axiom asserts that the range of it is the datatype $V$. The third axiom declares the role $A R_{i}$ is functional.

Example 4.3. Consider the attributive relation HospitalDescription in Table 1.7. Attribute HospitalType provides descriptions for the elements of the Hospital category, and is represented as a role HospitalType. Its domain is the Hospital category, and the range is String. The translation of the HospitalType attribute into the TBox is done through the following axioms:

$$
\begin{gathered}
\exists \text { HospitalType } \sqsubseteq \text { Hospital, } \quad \exists \text { HospitalType } \text { String, } \\
\geq 2 \text { HospitalType } \sqsubseteq \perp .
\end{gathered}
$$

The first axiom describes that the domain of the role HospitalType is the concept Hospital, and the second axiom states that its range is the datatype String. The third axiom asserts that each hospital is at most one type.

The partial order relation between the elements of categories in dimensions is represented by a role, say $P$. $P$ is made transitive by adding the following axiom to the TBox: $\operatorname{Tr} a(P)$, which asserts that $P$ must be interpreted as a transitive role.

Example 4.4. The partial order relation between the elements of the contextual dimension Location in Figure 3 is represented by a role $R U_{\text {Location }}$ which is made transitive by using the axiom: $\operatorname{Tr} a\left(R U_{\text {Location }}\right)$. Let an interpretation $\mathcal{I}=\left\langle\Delta^{\mathcal{I}}, .^{\mathcal{I}}\right\rangle$ have a domain as:

$$
\Delta^{\mathcal{I}}=\left\{\text { Suzan, } W_{1}, \text { standard }, H_{1}\right\}
$$


The role $R U_{\text {Location }}$ is translated by the interpretation function ${ }^{\mathcal{I}}$ as follows:

$$
\begin{aligned}
R U_{\text {Location }}= & \left\{\left(\text { Suzan }, \mathrm{W}_{1}\right),\left(\mathrm{W}_{1}, \text { standard }\right),\left(\text { standard }, \mathrm{H}_{1}\right),\right. \\
& \left.(\text { Suzan }, \text { standard }),\left(\text { Suzan }, \mathrm{H}_{1}\right),\left(\mathrm{W}_{1}, \mathrm{H}_{1}\right)\right\}
\end{aligned}
$$

If $C$ stands for a concept and $R$ stands for a role, $D L-L i t e_{H o r n}^{(H N)^{+}}$also offers the qualified number restriction $\geq q R . C$ ( $q$ is a positive integer), where the number restrictions are concerned with role-fillers belonging to a certain concept.

Consider the category $C_{k}$ is the parent of the category $C_{j}$ in a dimension $D\left(C_{j} \nearrow C_{k}\right)$. In addition, consider the category $C_{j}$ which in turn is the parent of the category $C_{i}$ in the dimension $D\left(C_{i} \nearrow C_{j}\right)$. Let these relations be introduced into the TBox as followings:

$$
\begin{aligned}
C_{k} & \sqsubseteq \exists P^{-} . C_{j} \\
C_{j} & \sqsubseteq \exists P^{-} . C_{i}
\end{aligned}
$$

$\exists P^{-} . C_{j}$ describes those pair of elements of $P$ that their domain is restricted to the concept $C_{j}$. In the same way, $\exists P^{-} . C_{i}$ describes those pair of elements of $P$ that their domain is restricted to the concept $C_{i}$. The (9) states that the concept $C_{k}$ is the subclass of the range of the role $P$, while the domain of $P$ is restricted to the concept $C_{j}$. Similarly, the (10) declares that the concept $C_{j}$ is the subclass of the range of the role $P$, while the domain of $P$ is restricted to the concept $C_{i}$.

By having the role $P$ transitive, the fact that the category $C_{k}$ is the parent of the category $C_{i}$ is logically implied by (9) and (10).

It worths to mention that for the homogeneous dimensions, in order to represent the partial order relation between the categories such as $C_{i}$ and $C_{j}$ that are connected as $C_{i} \nearrow$ $C_{j}$, it is possible to express the axiom $C_{i} \sqsubseteq \exists P . C_{j}$. See Section 5.1 for details. 
Example 4.5. As illustrated in Figure 3, in the Location dimension, Nurse and Ward categories are related as Nurse $\nearrow$ Ward. The partial order relation between the elements of these two categories is expressed by the following axiom:

$$
\text { Ward } \sqsubseteq \exists R U_{\text {Location. }}^{-} \text {Nurse }
$$

The above axiom states that elements of the concept Ward are the subclass of the range of the pairs whose their domain is concept Nurse. Note that we assume there is no loose element in the category Ward.

The $\nearrow$ relation between the elements of the other categories in the Location dimension is represented as follows:

$$
\begin{aligned}
\text { Care-Unit } & \sqsubseteq \exists R U_{\text {Location. }}^{-} \text {. Ward, } \\
\text { Hospital } & \sqsubseteq \exists R U_{\text {Location. }}^{-} \text {. Care-Unit, } \\
\text { City } & \sqsubseteq \exists R U_{\text {Location. }}^{-} \text {Hospital, } \\
\text { Country } & \sqsubseteq \exists R U_{\text {Location. }}^{-} \text {. City, } \\
\text { All-Location } & \sqsubseteq \exists R U_{\text {Location. }}^{-} \text {Country. }
\end{aligned}
$$

Note that, it is not possible to express the relation between the elements of the categories Ward and Care-Unit by the axiom $\exists R U_{\text {Location. }}$ Care-Unit $\sqsubseteq$ Ward. The reason is that the axiom states if the range of the each pair of elements in the $R U_{\text {Location }}$ role is restricted to the elements of the Care-Unit category, then the domain of those pairs should be the sub class of the Ward category. However, since the role $R U_{\text {Location }}$ is expressed as a transitive role, its domain also contains those elements of Nurse category that are related to the elements of the Care-Unit category.

Each categorical relation CR containing $n$ attributes such as $A_{1}, \ldots, A_{n}$ is represented as an atomic concept $C R C o n$ in the TBox $\mathcal{T}$. In addition, we also have atomic roles, such 
as $C R \operatorname{Con}_{A_{1}}, \ldots, C R C o n_{A_{n}}$ and the following inclusion assertions:

$$
\begin{aligned}
\exists C R \operatorname{Con}_{A_{1}} & \sqsubseteq A_{1}, \quad \ldots, \quad \exists \text { CRCon }_{A_{n}} \sqsubseteq A_{n} \\
\text { CRCon } & \sqsubseteq \exists C R \text { Con }_{\bar{A}_{1}}^{-}, \ldots, \quad \text { CRCon } \sqsubseteq \exists C R \text { Con }_{\bar{A}_{n}}^{-}
\end{aligned}
$$

Example 4.6. Consider the categorical relation PatientValueWard in Table 4.1 which is related to the Ward category of Location dimension and Hour category of Time dimension:

\begin{tabular}{|c|c|c|c|}
\hline \multicolumn{4}{|c|}{ Table 4.1: PatientValueWard } \\
\hline Patient & Value & Hour & Ward \\
\hline Tom Waits & 38.5 & $11: 45 / 5 / \mathrm{Sep} / 2011$ & $\mathrm{~W}_{1}$ \\
Tom Waits & 38.2 & $12: 10 / 5 / \mathrm{Sep} / 2011$ & $\mathrm{~W}_{1}$ \\
Tom Waits & 38.1 & $11: 50 / 6 / \mathrm{Sep} / 2011$ & $\mathrm{~W}_{1}$ \\
Tom Waits & 38.0 & $12: 15 / 7 / \mathrm{Sep} / 2011$ & $\mathrm{~W}_{1}$ \\
Tom Waits & $110 / 70$ & $11: 45 / 8 / \mathrm{Sep} / 2011$ & $\mathrm{~W}_{3}$ \\
Lou Reed & 37.9 & $12: 10 / 5 / \mathrm{Sep} / 2011$ & $\mathrm{~W}_{2}$ \\
\hline
\end{tabular}

The relation PatientValueWard is translated into the TBox as a concept PWCon. In order to represent the attributes of it in the TBox, roles $P W_{P a t i e n t}, P W_{\text {Value }}, P W_{\text {Hour }}$ and $P W_{W a r d}$ are introduced. The following inclusions are held for the previously introduced 
concept and roles:

$$
\begin{array}{rlrl}
\exists P W_{\text {Patient }} & \sqsubseteq \text { Patient, } & \exists P W_{\text {Value }} \sqsubseteq \text { Value }, \\
\exists P W_{\text {Hour }} \sqsubseteq \text { Hour, } & \exists P W_{\text {Ward }} \sqsubseteq \text { Ward } \\
P W C o n & \sqsubseteq \exists P W_{\text {Patient }}^{-}, & \text {PWCon } \sqsubseteq \exists P W_{\text {Value }}^{-}, \\
P W C o n & \sqsubseteq \exists P W_{\text {Hour }}^{-} & \text {PWCon } \sqsubseteq \exists P W_{\text {Ward }}^{-}
\end{array}
$$

\subsection{Translating a Contextual Dimension Instance into a DL ABox}

As previously mentioned in Chapter 2, the data of the DL-Ontology is maintained in the ABox, $\mathcal{A}$, of the ontology. The ABox is perfectly compatible with the TBox, which means that the vocabulary of concepts, roles, and attributes in $\mathcal{A}$ is those used in the TBox. The ABox stores abstract objects which are returned as answers for queries over the ontology.

When the ABox is very large, the ontology system requires to manage data in the secondary storage. Accordingly, we are going to store the possible dimensions' instances in the data source. In this way, in order to link the existing data in data sources to the instances of the concepts and the roles in the ontology, the set of mapping assertions are defined. Through each mapping a conjunctive query over atomic concepts, roles and attributes is associated with a first-order query of the appropriate arity over the data source. The intuition is that, by evaluating such a query, the facts for constituting the ABox assertions for the concepts and roles appearing in the conjunctive query, is retrieved. The approach for linking the existing data in data sources to the instances of the concepts and the roles in the ontology is discussed in Section 2.5.1. In the following, the notion of mappings is discussed by means of an example.

Example 4.7. Assume we have stored the Location dimension instance which is illustrated 
in Figure $3 \mathrm{~b}$ as a relation LocationInstance(Nurse, Ward, Care-Unit, Hospital, City, Country) in the data source. Consider the represented concepts and roles in the TBox in the Example 4.1. We want to model the situation where $w \in$ Location Instance corresponds to a ward whose name is $w$. Similarly, we want to model the fact that $c u \in$ LocationInstance corresponds to the name of the care-unit that the ward located in. The following is the set of mapping assertions modeling the above situation.

$$
\begin{aligned}
\forall n \forall w \forall c u \forall h \forall c i \forall c o(\text { LocationInstance }(n, w, c u, h, c i, c o) & \left.\rightarrow \operatorname{Ward}\left(f_{\text {ward }}(w)\right)\right) \\
\forall n \forall w \forall c u \forall h \forall c i \forall c o(\text { LocationInstance }(n, w, c u, h, c i, c o) & \rightarrow \\
& \text { Care-Unit } \left.\left(f_{\text {care-unit }}(c u)\right)\right)
\end{aligned}
$$

Above, $w$ and $c u$ are variable symbols; $f_{\text {ward }}$ and $f_{\text {care-unit }}$ are function symbols; and $f_{\text {ward }}(w)$ and $f_{\text {care-unit }}(c u)$ are variable object terms.

Consider the attributive relation HospitalDescription (Table 1.7), the following mapping creates instances of a role HospitalType in the DL ontology.

$$
\forall h \forall t \forall s\left(\text { HospitalDescription }(h, t, s) \rightarrow \text { HospitalType }\left(f_{\text {hospital }}(h), t\right)\right)
$$

In this case, the Type of HospitalDescription (a string) contains data value constants which are mapped as a data value constants at the ontological level.

Consider the role $R U_{\text {Location }}$ which stands for the partial order relation between the elements of the Location dimension. The following mappings assert the pair of instances to the role $R U_{\text {Location }}$ :

$$
\begin{aligned}
\forall n \forall w \forall c u \forall h \forall c i \forall c o(\text { LocationInstance }(n, w, c u, h, c i, c o) & \rightarrow \\
& \left.R U_{\text {Location }}\left(f_{\text {ward }}(w), f_{\text {care-unit }}(c u)\right)\right)
\end{aligned}
$$


$\forall n \forall w \forall c u \forall h \forall c i \forall c o($ LocationInstance $(n, w, c u, h, c i, c o) \rightarrow$

$$
\left.R U_{\text {Location }}\left(f_{\text {care-unit }}(c u), f_{\text {hospital }}(h)\right)\right)
$$

the mapping (11) asserts the pairs of the instances with the domain as the Ward category and the range as the Care-Unit category to the role $R U_{\text {Location }}$. The mapping (12) asserts the pairs of the instances with the domain as the Care-Unit category and the range as the Hospital category. Since the role $R U_{\text {Location }}$ is declared as a transitive role, the fact that the role $R U_{\text {Location }}$ also contains the pairs of the elements with the domain as the Ward category and the range as the Hospital category is logically implied.

The represented contextual dimensions in DL-ontology provide data from detailed to more general. We are able to navigate through the dimensions and obtain more detailed and general information through querying the TBox. We consider conjunctive queries over the DL-ontology for querying the TBox. In Chapter 6, we show how the ontological representation of contextual dimensions can be used for obtaining the quality instances. 


\section{Chapter 5}

\section{Ontological Representation of Semantic}

\section{Constraints}

As discussed in Chapters 1 and 2, the data under quality assessment is put in a context $\mathfrak{C}$, that contains additional information to those data that are under quality assessment. This additional information can be local data at $\mathfrak{C}$, additional semantic constraints, etc.

In DL ontologies, ICs can be expressed through axioms in a TBox $\mathcal{T}$. A DL-ontology is satisfiable if there exists an interpretation $\mathcal{I}$ that satisfies all the assertions in TBox $\mathcal{T}$ and $\mathrm{ABox} \mathcal{A}$. This interpretation is called a model of the ontology. The models of the $\mathrm{DL}$ ontology should satisfy all the assertions in $\mathcal{T}$; hence ICs that are expressed as $\mathcal{T}$ 's axioms should be satisfied by all of the models of the ontology.

In the following, we discuss the representation of the $\mathcal{M D}$ constraints such as homogeneity, and intra- and interdimensional semantic constraints as axioms in a DL-ontology. Furthermore, in the rest of this chapter, we describe the translation of the hard rules such as guidelines into the DL-ontology.

\subsection{Ontological Representation of Homogeneity}

Assume $C_{i}$ and $C_{j}$ are two categories in a dimension $D$ which are connected as $C_{i} \nearrow C_{j}$. If the categories $C_{i}$ and $C_{j}$ are translated into the DL-ontology as concepts $C_{i}$ and $C_{j}$ 
respectively, and the role $P$ stands for the partial order relation between the elements of $D$ (See Section 4.1 for details), the homogeneity condition is captured by introducing the following axiom in the $\mathcal{T}$ :

$$
C_{i} \sqsubseteq \exists P . C_{j}
$$

Example 5.1. Consider the Location dimension in Figure 3. Let $R U_{\text {Location }}$ be a role which stands for the partial order relation between the elements of the Location dimension. Homogeneity is captured through the following axioms in the $\mathcal{T}$ :

$$
\begin{aligned}
\text { Nurse } & \sqsubseteq \exists R U_{\text {Location.Ward, }}, \\
\text { Ward } & \sqsubseteq \exists R U_{\text {Location. Care-Unit, }} . \quad \exists R U_{\text {Location. Hospital, }}, \\
\text { Care-Unit } & \sqsubseteq \exists R U_{\text {Location. }} \text {. ity, } \\
\text { Hospital } & \sqsubseteq \exists R U_{\text {Location. Country, }} \\
\text { City } & \sqsubseteq \exists R U_{\text {Location. All-Location }}
\end{aligned}
$$

The representation of the homogeneity constraint as axioms in the ontological context ensures that whenever the contextual roll-up for obtaining the quality instances is needed, it is always possible to obtain the more general elements from the detailed ones.

\subsection{Ontological Representation of Contextual Guidelines}

Contextual guidelines are hard integrity constraints in a context that are enforced and must always be satisfied. They are represented as axioms in a TBox. The representation of contextual guidelines as axioms in a DL ontology is illustrated and discussed in Example 5.2. 
Example 5.2. Contextual guidelines, e.g. Hospital Guideline 2 stating that "medical tests on patients in standard care units have to be taken with instruments made by manufacturer $M_{1}$ ", can be captured as axioms in the TBox $\mathcal{T}$. As discussed in Chapter 4 , each category is represented as a concept in the TBox and elements of the categories are represented as instances of the concepts in the ABox. In order to consider the standard element of the Care-Unit concept and the element $M_{1}$ of the Manufacturer category in the axioms, we first need to introduce two concepts, StandardCon and $\mathrm{M}_{1}$ Con, respectively containing the element standard, and the element $\mathbf{M}_{1} .{ }^{1}$ That is, $\mathbf{M}_{1} \operatorname{Con}\left(\mathbf{M}_{1}\right)$ and StandardCon(standard) are facts in the ontology. These two new concepts satisfy the following conditions:

$$
\text { StandardCon } \sqsubseteq \text { Care-Unit, } \mathbf{M}_{1} \text { Con } \sqsubseteq \text { Manufacturer. }
$$

We introduce a new concept StandardRelate that contains all the locations that have standard as an ancestor in the Care-Unit category. StandardRelate satisfies the following constraint:

$$
\text { StandardRelate } \equiv \exists R U_{\text {Location }} . \text { StandardCon, . }
$$

Similarly, $\mathrm{M}_{1}$ Relate is a concept with instruments with $\mathrm{M}_{1}$ as an ancestor in the Manufacturer category. $\mathbf{M}_{1}$ Relate satisfies the following constraint:

$$
\mathbf{M}_{1} \text { Relate } \equiv \exists R U_{\text {Instrument }} \cdot \mathbf{M}_{1} \text { Con }
$$

Finally, a new role UnitIns is considered, which relates the instruments that are used in medical tests to the locations that the medical tests has performed. The contextual guideline 2 can be expressed as an axiom using UnitIns as:

\section{$\exists$ UnitIns $^{-}$.StandardRelate $\sqsubseteq \mathrm{M}_{1}$ Relate}

the above expression states that if a medical test is performed in one of the locations related to the standard care-unit, the instrument which is used in the test, is made by manufacturer

\footnotetext{
${ }^{1}$ Since axioms are constructed by using concepts and roles not the instances of the concepts and roles.
} 
$M_{1}$

\subsection{Ontological Representation of Interdimensional Constraints}

Interdimensional constraints are introduced in Section 3.2.2. They can be captured as axioms in the TBox. The representation of interdimensional constraints in the DL ontology is discussed and illustrated in the Example 5.3.

Example 5.3. Consider the category Hospital in the Location dimension. The information about the 'day' that hospitals in the Hospital categories were founded appears in a relation HospitalFounded in Table 6.1 (Figure 11).

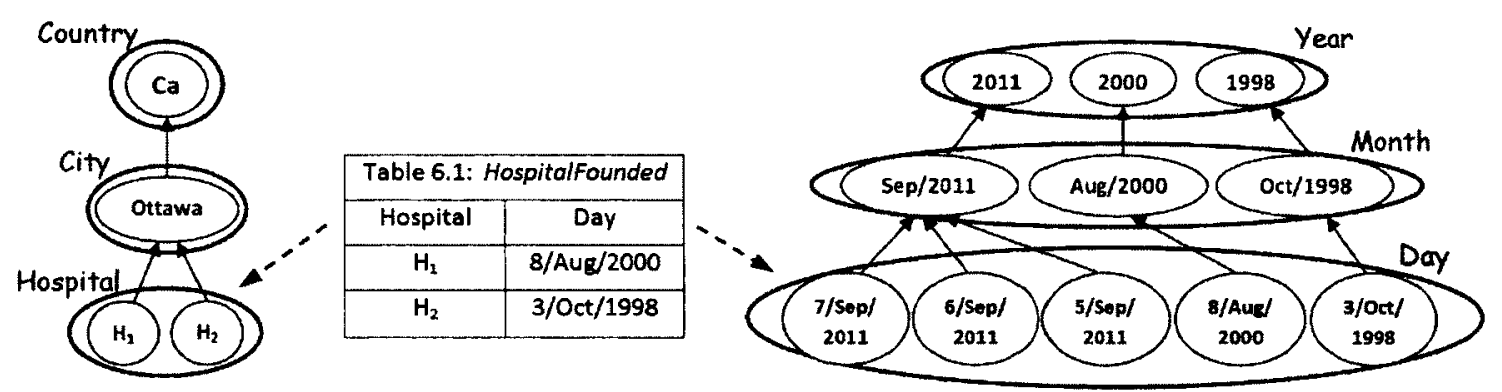

Figure 11: HospitalFounded to Location and Time Mappings

We know if the hospital is located in the country USSR, the date of its creation can not be after 1991. This condition can be expressed as an interdimensional constraint, because it involves two dimensions. In order to do this, we first need to introduce a concept Before-1991, containing the years from the Year category before 1991. The new concept satisfies the following constraint:

$$
\text { Before-1991 } \sqsubseteq \text { Year. }
$$

In addition, we need to introduce the concept USSRCon containing the element USSR 
from the Country category. USSRCon satisfies the following constraint:

$$
\text { USSRCon } \sqsubseteq \text { Country. }
$$

We introduce the concept USSRRelate that contains all the locations having USSR as an ancestor in the Country category. Similarly, DatesBefore1991 is a concept with dates with year before 1991 as an ancestor in the Year category. These new concepts satisfy the conditions:

$$
\text { USSRRelate } \equiv \exists R U_{\text {Location. }} . \text { USSRCon, } \quad \text { DatesBefore1991 } \equiv \exists R U_{\text {Time }} . \text { Before-1991 }
$$

Finally, the interdimensional constraint can be expressed using the role HospitalFounded as:

$$
\exists \text { HospitalFounded }{ }^{-} \text {.USSRRelate } \sqsubset \text { DatesBefore1991 }
$$

the axiom (13) states that those locations that have USSR as an ancestor in the Country category are established before 1991 .

\subsection{Ontological Representation of Intradimensional Constraints}

Intradimensional constraints are introduced in Section 3.2.1. They can also be captured as axioms in the TBox. The representation of intradimensional constraints in the DL-ontology is discussed in Example 5.4.

Example 5.4. Hospital $H_{2}$ is a small hospital that accepts patients only during working days. Every year, for renovation, the hospital would be closed and no patient is accepted.

Consider relation WorkingDaysCalendar in Table 6.2. It contains information about the days of the year 2011 that the hospital is closed.

\begin{tabular}{|c|c|}
\hline \multicolumn{2}{|c|}{ Table 6.2: WorkingDaysCalendar } \\
\hline Day & Calendar \\
\hline 12/Sep/2011 & Holiday \\
24/Oct/2011 & Holiday \\
$\ldots$ & $\cdots$ \\
\hline
\end{tabular}




\begin{tabular}{|c|c|}
\hline \multicolumn{2}{|c|}{ Table 6.3: RenovationTimes } \\
\hline Month & Calendar \\
\hline April & Closed \\
May & Closed \\
\hline
\end{tabular}

In addition, consider relation NotWorkingMonthsCalendar in Table 6.3. It contains information about the month of the year 2011 when the hospital is closed for the renovation.

When in one month the hospital is closed for renovation, in all of the days of that month, the hospital is closed. Based on the relation between the Day category and the Month category in the Time dimension in Figure 10, the above condition can be expressed as an intradimensional constraint, that involves different categories of a single dimension.

Intradimensional constraints can be captured as axioms in DL ontology. In order to represent the previously mentioned condition as axioms in DL, two new roles Holidays and RenovationTimes are introduced. Holidays and RenovationTimes contain information about holidays and the months that the hospital is closed for the renovation, respectively. In addition, we also introduce a new concept ClosedMonth equivalent to the domain of the role RenovationTimes containing the months when the hospital is closed for renovation. It satisfies the following constraint:

\section{$\exists$ Renovation Times $^{-} \equiv$ ClosedMonth}

The following axiom stands for the previously mentioned intradimensional constraint:

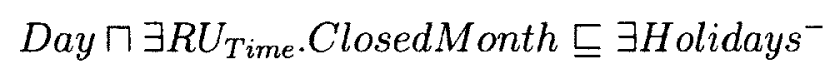

the axiom 14 states that days of the months which the hospital is closed for the renovation, are also holidays.

We have discussed the representation of the contextual dimensions into a DL-ontology in Chapter 5. Furthermore, we represented the contextual guidelines, the intra - and interdimensional semantic constraints as axioms in the DL-ontology. As a result of the ontological 
representation of the context, logical reasoning could be performed for extracting implicit information. In the following, we show how the ontological representation of the context can be used for assessing the quality of data by means of an example.

Example 5.5. Tom is a patient in a hospital. Several times a day different medical tests are performed on him, and his results are recorded. His doctor, John, wants to see Tom's test values every day, to follow his evolution.

The data that John needs about Tom appear, among other, in the Patient Value relation in Table 1.1 (Page 3). John has a quality concern. He asks nurses to perform all the medical tests with instruments made by manufacturer $M_{1}$. The context has information about hospital guideline:

Contextual Hospital Guideline 2: 'Medical tests on patients in standard care unit has to be taken with instruments made by manufacturer $M_{1}^{\prime}$ (Page 8).

In addition to the hospital guideline, in contextual relation PatientValueWard (Table 4.1, Page 51), we have the information about the medical tests results and the wards that the tests has performed in them.

The information explicitly provided by the contextual relation PatientValueWard is about the Ward category, but data about the Care-Unit, that could be used in combination with Guideline 2, belongs to a higher or more general category.

We have discussed the translation of the contextual dimension Location into the DLontology in Chapter 4 (Example 4.1, 4.2, 4.3, 4.5). In addition, we also translated the contextual relation PatientValueWard into the DL-ontology in Example 4.6. Furthermore, previously in this chapter, we discussed the representation of the contextual guideline 2 in the TBox. Having the ontological representation of the contextual relation PatientValueWard (Table 4.1, Page 51), and the ontological representation of the Contextual Hospital Guideline 2, the following query asks about those tests' results of the patients that are taken in the standard care-unit. The query is written in terms of the concepts and roles of 
the ontology, and is expressed as the conjunctive queries, in particular Datalog [21].

$$
\begin{aligned}
\mathcal{Q}(p, v, h) \leftarrow & P W_{\text {Patient }}(p w, p), P W_{\text {Value }}(p w, v), P W_{\text {Hour }}(p w, h), \\
& P W_{\text {Ward }}(p w, w), \operatorname{StandardRelate}(w),
\end{aligned}
$$

where the roles $P W_{\text {Patient }}, P W_{\text {Hour }}, P W_{\text {Value }}$ and $P W_{W a r d}$ are the roles of the ontology which are introduced for the translation of the contextual relation PatientValueWard (Table 4.1, Page 51) into the TBox, StandardRelate is the concept of the ontology which contains all the instances of the Location dimension that has the element standard as an ancestor in the Care-Unit category (previously introduced for the representation of the Hospital Guideline 2 into the ontology in Example 5.2, Page 57).

The above query is asking about the test results of patients that are taken in the locations which have Standard as an ancestor in the Care-Unit category at different times. The concept StandardRelate is equivalent to the domain of the role RULocation when the range of the role RULocation, is restricted to the concept StandardCon (the concept that contains the element Standard, see Example 5.2, Page 57 for details). The set of axioms of the ontological representation of the Hospital Guideline 2 declare that the instruments that are used in one of locations related to the concept StandardRelate are made by the manufacturer $M_{1}$. If the ontology is satisfiable, by logical reasoning the navigation through the hierarchy is done for obtaining all the locations that have the element Standard as an ancestor in the Care-Unit category. Those values of the tests' results that are performed in the wards belong to the StandardRelate category are returned as the certain answers to the query $\mathcal{Q}$. The certain answers to the query $\mathcal{Q}$ (quality instance) is illustrated in Table 6.4. 


\begin{tabular}{|c|c|l|}
\hline \multicolumn{3}{|c|}{ Table 6.4: Certain Answers } \\
\hline Patient & Value & \multicolumn{1}{c|}{ Hour } \\
\hline Patient-Tom Waits & Value-38.5 & Hour-11:45/5/Sep/2011 \\
Patient-Tom Waits & Value-38.2 & Hour-12:10/5/Sep/2011 \\
Patient-Tom Waits & Value-38.1 & Hour-11:50/6/Sep/2011 \\
Patient-Tom Waits & Value-38.0 & Hour-12:15/7/Sep/2011 \\
Patient-Lou Reed & Value-37.9 & Hour-12:10/5/Sep/2011 \\
\hline
\end{tabular}

The several possible contextual instance may become admissible candidates to be used for data quality assessment. The quality of relation PatientValue (Table 1.1) is assessed using the quality measure $q m_{1}$ which is introduced in [16] (See Section 2.2, Page 21 for details):

$$
q m_{1}(D):=\frac{|D|-\max \left\{\left|D_{\mathcal{P}}^{\prime}(I)\right|: I \text { is } \quad \text { LCI }\right\}}{|D|}
$$

The number of tuples of the relation PatientValue which is under quality assessment $(|D|)$ is 6 and the number of the certain answers to the query $\mathcal{Q}\left(\left|D_{\mathcal{P}}^{\prime}(I)\right|\right)$ is 5 . The quality measure $q m_{1}$ is evaluated to 0.17 which means the data in the relation PatientValue are almost quality data (since the value is near to 0 ). 


\section{Chapter 6}

\section{Experiments}

\subsection{Introduction}

In Chapters 4 and 5, we introduced axioms for translating the extended $\mathcal{M D}$ model into a description logic ontology. More precisely, we did the representation in a member of the

$D L$-Lite family of DLs, $D L-L i t e_{H o r n}^{(\mathcal{H N})^{+}}$. We have also introduced axioms for representing the semantic constraints over the extended $\mathcal{M D}$. In this experiment the proposed axioms are written in the language of ontology web language (OWL), more precisely OWL 2 [47]. OWL 2 offers the representation of the classes, roles and datatypes.

OWL represents the class Nothing as a built-in classes with a predefined semantic. The class Nothing is compatible with the empty class $(\perp)$ in DL-Lite. An axiom Subclassify $\left(C_{1}, C_{2}\right)$ in OWL stands for the subclass axiom of $D L$-Lite for concepts $C_{1}$ and $C_{2}\left(C_{1} \sqsubseteq C_{2}\right)$. An intersection class axiom in OWL IntersectionOf( $\left.C_{1}, C_{2}\right)$ represents the conjunction of classes $C_{1}$ and $C_{2}\left(C_{1} \sqcap C_{2}\right)$ in DL-Lite. An inverse object properties axiom Inverse Of $\left(R_{1}\right)$ in OWL corresponds to the inverse role property $\left(R^{-}\right)$in DL-Lite. A minimum cardinality construct in OWL objectMinCardinality $(n R)$ stands for the number restriction of the form $\geq n R$ in $D L-L i t e^{\mathcal{N}}$. An object property transitivity axiom TransitiveProperty $(R)$ corresponds to the $\operatorname{Tr} a(R)$ axiom for the transitive role property in $D L$ Lite ${ }^{(\mathcal{H N})^{+}}$. The SomeValuesFrom class construct stands for the existential quantification 
$(\exists R . C)$ in $D L-L i t e^{(\mathcal{H N})^{+}}$.

In this chapter, we show an experiment on representing our extended $\mathcal{M D}$, including semantic constraints in OWL. The experiment is based on the previously proposed rules. We will check the satisfiability of the resulting ontology, and also we will perform query answering over it for data quality assessment. We verify the satisfiability of the resulting ontology by the Pellet reasoner [54] (e.g. the reasoner verifies whether the concepts that are declared as disjoint concepts have any common instances or not).

The experiment is based on our running example about the different medical tests on patients in a hospital (cf. Example 1.1, Page 3). We consider contextual dimensions Location, Instrument and Time. The schema for these contextual dimensions can be found in Figures $3 \mathrm{a}, 6 \mathrm{a}$ and 10 , respectively.

We consider three relations Instrument, Location and Time which contain contextual dimensions instances with the following schemas:

$$
\begin{array}{r}
\text { Instrument (model, brand, manu facturer, type, class) } \\
\text { Location(nurse, ward, unit, hospital, city, country) } \\
\text { Time(hour, day, month, shift, week, year) }
\end{array}
$$

In addition, we have the following categorical and attributive relations:

$$
\begin{array}{r}
\text { PatientValueWard(patient, value, hour, ward) } \\
\text { HospitalDescription (hospital, type, system) }
\end{array}
$$

In this experiment the following concepts, roles are involved:

Concepts: Each concept in OWL is represented by the construct: <owl:Class $>$.

- Each of the categories in a dimension are represented by a concept name in OWL. For example the categories of the Location dimension (Figure 3a) are introduced by the concepts: Nurse, Ward, Care-Unit, Hospital, City, Country and All-Location. 
- In order to represent the disjointness between the categories of each dimension in OWL, a concept which is equivalent to the conjunction of each two concepts (which are the representation of the categories of the dimension), is introduced. These concepts include the followings: NurseWard, NurseCare-Unit, NurseHospital, NurseCity, NurseCountry, WardCare-Unit, ...

- For representing the Hospital Guideline 2 (Page 8) in OWL, the following concepts are considered: MICon, StandardCon, MIRelate and StandardRelate.

- In order to translate the relation PatientValueWard (Page 51) into the OWL, the concept $P W$ is introduced.

DataTypeProperty: Each datatype property (a role with the domain as a concept and range as a datatype) in OWL is represented by the axiom: <owl:DatatypeProperty>.

- The datatype properties HospitalType and HospitalSystem are considered for representing the attributive relation HospitalDescription (Table 1.7 in Example 1.6, Page 10) in the TBox.

Roles: Each role in OWL is expressed by the construct: <owl:ObjectProperty>.

- The partial order relation between the elements of each dimension in OWL is represented by an object property: RUTime, RULocation and RUInstrument.

- In order to represent the Hospital Guideline 2 (Page 8) in OWL, the object property UnitIns is introduced.

- In order to represent the categorical relation PatientValueWard (Table 4.1, Page 51) in OWL, the following object properties are introduced: PWPatient, PWValue, PWHour and PWWard. 
In this experiment, for representing the context as an ontology in OWL, the Protègè software [40] is used. Protègè is a free, open-source platform that implements a rich set of knowledge-modeling structures and actions that support the creation, visualization, and manipulation of ontologies in various representation formats. The relations containing dimensions' instances are defined in PostgreSQL [51].

The set of mappings for generating the ABox assertions is represented in Turtle syntax [11]. A Turtle document allows writing down an RDF graph in a compact textual form. An RDF graph is made up of triples consisting of a subject, predicate and object. The assertions in the ABox are generated from the set of the mappings written in Turtle syntax by using the Quest system [2].

\subsection{Axioms in the TBox}

At the first step, we considered a concept (an OWL class) for each of the categories in a dimension. Consider the dimension Location, the following code represents class Hospital which stands for the Hospital category in the Location dimension:

<!- http://www.semanticweb.org/thesis.owl\#Hospital ->

<owl:Class rdf:about="http://www.semanticweb.org/thesis.owl\#Hospital"/>

The above representation states that under the namespace "http://www.semanticweb.org/thesis.owl", there is a concept Hospital.

As shown in Chapter 4, categories are assumed to be disjoint. In other words, the conjunction of each two categories should be empty. We represent the disjointness between each two categories in a dimension using the 'empty set' concept. The 'empty set' concept is represented in OWL by a built-in class owl:Nothing.

In order to represent the intersection of the concepts Hospital and Care-Unit in the Location dimension is empty, we introduce concept HospitalCare-Unit which is equivalent to the intersection of the Hospital and the Care-Unit concepts (As introduced in Section 2.5.1 
(Page 27), the conjunction of concepts is a new concept). The concept HospitalCare-Unit is the subclass of the concept owl:Nothing. The concept HospitalCare-Unit is represented as follows:

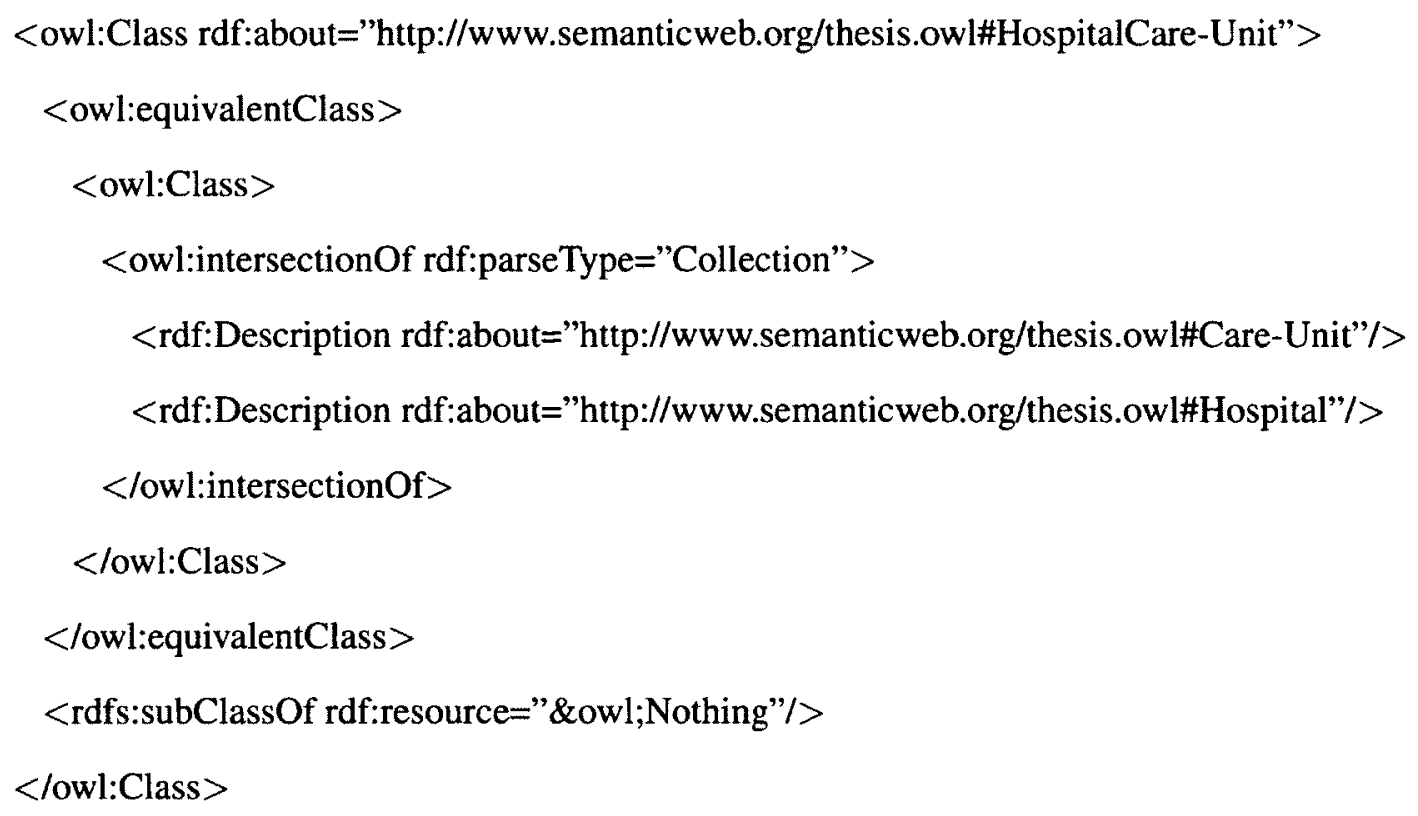

The above representation states that under the namespace "http://www.semanticweb.org/thesis.owl", a new concept HospitalCare-Unit is introduced which is equivalent to the intersection of the concepts Care-Unit and Hospital and is the subclass of the built in concept Nothing.

Each attribute in an attributive relation is translated as a role relating the elements of a category to a data value. Consider the attributive relation HospitalDescription in Table 1.7 (Example 1.6, Page 10) providing description for the elements of the Hospital category in the Location dimension with the following schema:

\section{HospitalDescription (Hospital, Type, System)}

The datatype property HospitalType describes the type of the hospitals of the Hospital category and is represented as follows: 
$<$ !- http://www.semanticweb.org/thesis.owl\#HospitalType $\rightarrow>$

<owl:DatatypeProperty rdf:about="http://www.semanticweb.org/thesis.owl\#HospitalType"> <rdfs:domain rdf:resource="http://www.semanticweb.org/thesis.owl\#Hospital"/>

<rdfs:range rdf:resource="\&xsd;string"/>

$<$ /owl:DatatypeProperty $>$

The above representation states that under the namespace "http://www.semanticweb.org/thesis.owl", the datatype property HospitalType has the domain as Hospital and the range as the datatype String.

The partial order relation between the elements of the categories of a dimension is represented in the TBox by a role which is transitive.

In our experiment, consider the contextual dimension Location, the partial order relation between the elements of its categories is represented in OWL by the object property RULocation. The object property RULocation has the following properties:

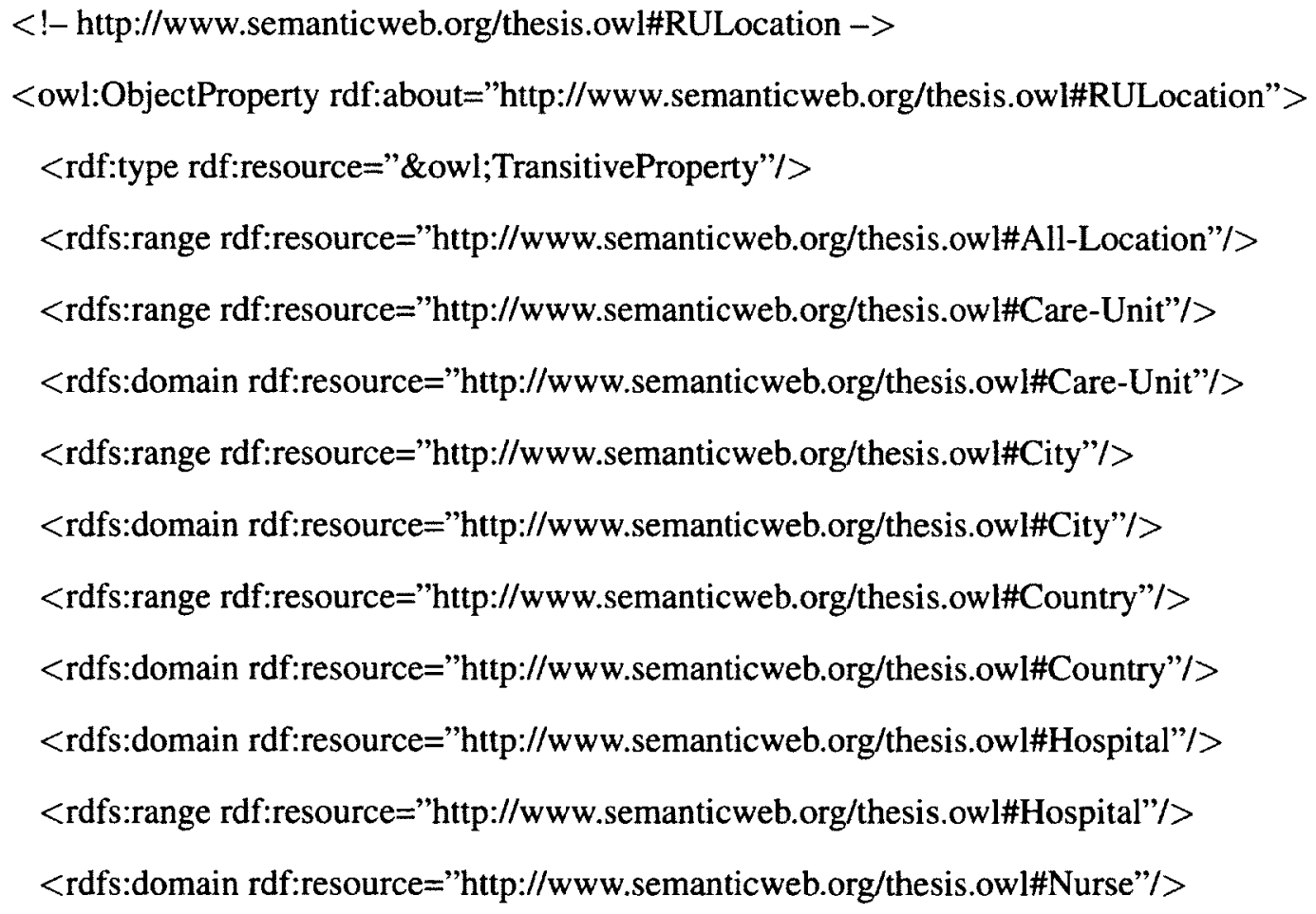


<rdfs:range rdf:resource="http://www.semanticweb.org/thesis.owl\#Ward"/>

<rdfs:domain rdf:resource="http://www.semanticweb.org/thesis.owl\#Ward"/> $<$ lowl:ObjectProperty $>$

The above declarations state that under the namespace "http://www.semanticweb.org/thesis.owl", the object property RULocation is transitive and has the domain as all of the concepts which stand for the categories of the Location dimension except the All-Location concept (top category). The role RULocation has a range as all of the concepts which stand for the categories of the Location dimension except the Nurse concept (bottom category).

The object property InvRULocation is declared as an inverse of the object property RULocation:

$<!-$ http://www.semanticweb.org/thesis.owl\#InvRULocation $->$

<owl:ObjectProperty rdf:about="http://www.semanticweb.org/thesis.owl\#InvRULocation"> <owl:inverseOf rdf:resource="http://www.semanticweb.org/thesis.owl\#RULocation"/> $</$ owl:ObjectProperty $>$

Consider the categories Ward and Care-Unit of the Location dimension. If they are connected as Ward $\nearrow$ Care-Unit, and the role RULocation stands for the partial order relation between the instances of the categories of the Location dimension, the relation between the categories Ward and Care-Unit is represented in OWL as follows:

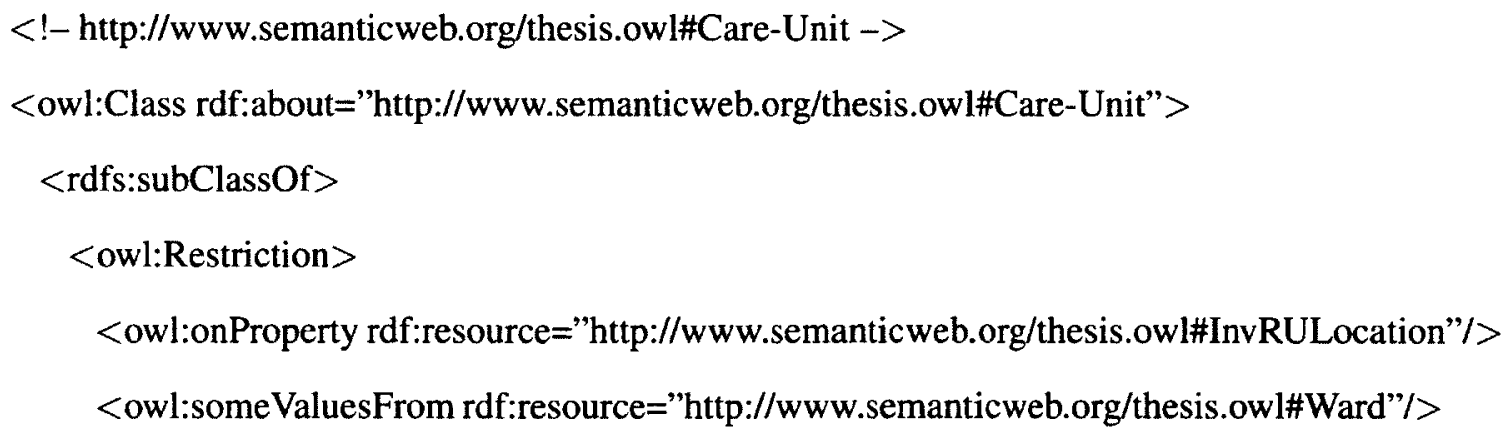




$$
\begin{aligned}
\quad</ \text { owl:Restriction }> \\
</ \text { rdfs:subClassOf }> \\
<\text { /owl:Class }>
\end{aligned}
$$

The above representation describes that if the range of the role RULocation is the concept Care-Unit then its domain should include the concept Ward instances.

The schema of the contextual dimensions Instrument (Figure 6a) and the schema of the contextual dimension Time (Figure 10) are also represented in OWL in a same way.

The categorical relation PatientValueWard (Table 4.1, Page 51) contains data about all the patients and the wards they were staying each day. In Example 4.6, we showed how the categorical relation PatientValueWard is represented as a concept in DL. The representation of the relation PatientValueWard as the concept $P W$ in OWL is as follows:

$<!-$ http://www.semanticweb.org/thesis.owl\#PW $\rightarrow>$

<owl:Class rdf:about="http://www.semanticweb.org/thesis.owl\#PW"/>

The above representation states that under the namespace "http://www.semanticweb.org/thesis.owl", there is a concept $P W$.

In order to represent the relation PatientValueWard into the DL, object properties $P W$ Patient, PWWard, PWValue and PWHour are introduced in Example 4.6 (Page 51). These object properties are as follows:

$<$ !- http://www.semanticweb.org/thesis.owl\#PWPatient $->$

<http://www.semanticweb.org/thesis.owl\#PWPatient">

<rdfs:domain rdf:resource="http://www.semanticweb.org/thesis.owl\#PW"/>

<rdfs:range rdf:resource="http://www.semanticweb.org/thesis.owl\#Patient"/>

$<$ lowl:ObjectProperty $>$

The above representations declares that under the namespace "http://www.semanticweb.org/thesis.owl", there is an object property PWPatient with the 
domain as the $P W$ concept and the range as the Patient concept.

$$
\begin{aligned}
& \text { <!- http://www.semanticweb.org/thesis.owl\#PWValue }-> \\
& \text { < owl:ObjectProperty rdf:about="http://www.semanticweb.org/thesis.owl\#PWValue"> } \\
& \text { <rdfs:domain rdf:resource="http://www.semanticweb.org/thesis.owl\#PW"/> } \\
& \text { <rdfs:range rdf:resource="http://www.semanticweb.org/thesis.owl\#Value"/> } \\
& \text { </owl:ObjectProperty> }
\end{aligned}
$$

The above representations declares that under the namespace "http://www.semanticweb.org/thesis.owl", there is an object property PWValue with the domain as the $P W$ concept and the range as the Value concept.

$$
\begin{aligned}
& \text { <!- http://www.semanticweb.org/thesis.owl\#PWHour }-> \\
& \text { <owl:ObjectProperty rdf:about="http://www.semanticweb.org/thesis.owl\#PWHour"> } \\
& \text { <rdfs:range rdf:resource="http://www.semanticweb.org/thesis.owl\#Hour"/> } \\
& \text { <rdfs:domain rdf:resource="http://www.semanticweb.org/thesis.owl\#PW"/> } \\
& \text { </owl:ObjectProperty> }
\end{aligned}
$$

The above representations declares that under the namespace "http://www.semanticweb.org/thesis.owl", there is an object property PWHour with the domain as the $P W$ concept and the range as the Hour concept.

$$
\begin{aligned}
& \text { <!- http://www.semanticweb.org/thesis.owl\#PWWard }-> \\
& \text { <owl:ObjectProperty rdf:about="http://www.semanticweb.org/thesis.owl\#PWWard"> } \\
& \text { <rdfs:domain rdf:resource="http://www.semanticweb.org/thesis.owl\#PW"/> } \\
& \text { <rdfs:range rdf:resource="http://www.semanticweb.org/thesis.owl\#Ward"/> } \\
& \text { </owl:ObjectProperty> }
\end{aligned}
$$

The above representations declares that under the namespace "http://www.semanticweb.org/thesis.owl", there is an object property PWward with the domain as the $P W$ concept and the range as the Ward concept. 


\subsubsection{Representation of Semantic Constraints}

In Chapter 5, we introduced axioms for representing the semantic constraints over the contextual dimensions. In particular, we proposed rules for representing homogeneity, contextual guidelines, interdimensional constraints and intradimensional constraints.

The translation of the Contextual Guideline 2 (Page 8) as an axiom into the TBox is discussed in Example 5.2 (Page 57).

In order to add the axioms for the representation of the Contextual Guideline 2 in OWL, first, we need to introduce a subclass of the concept Manufacturer and a subclass of the concept Care-Unit which are concepts $\mathrm{M}_{1}$ Con and StandardCon, respectively. See Page 57 for details:

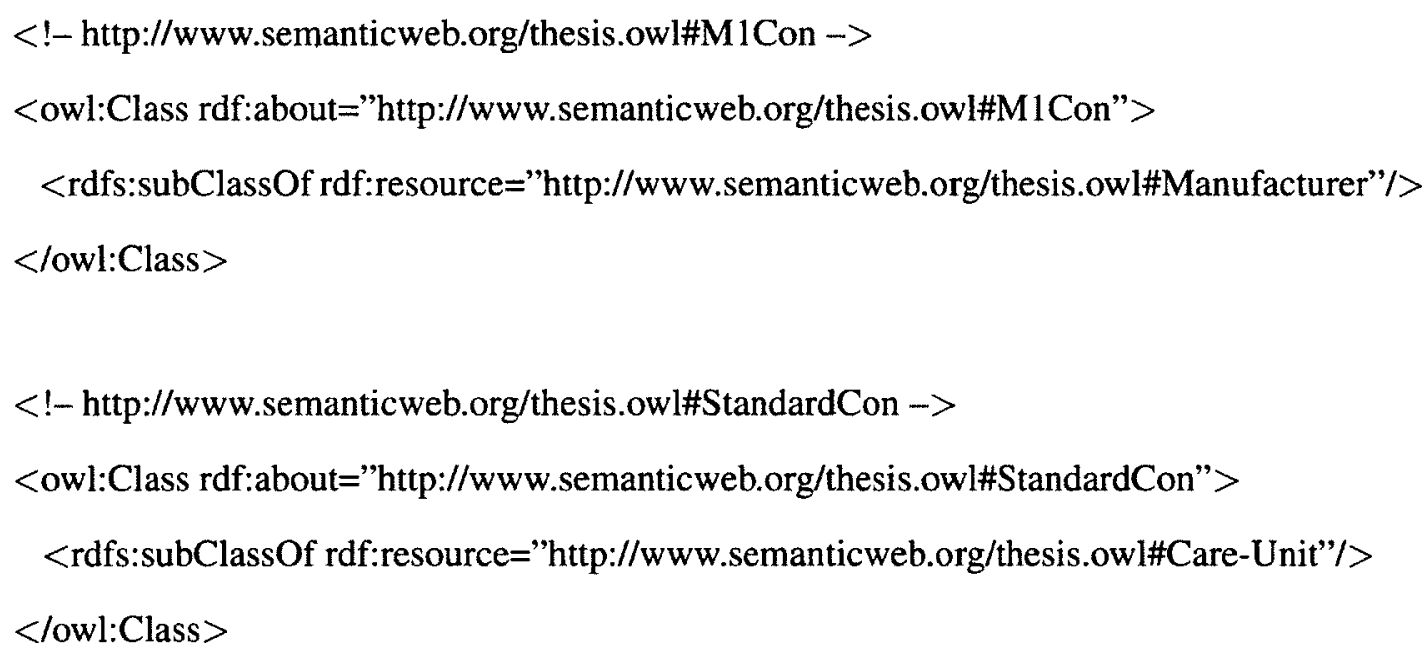

As discussed on Page 57, we also need to introduce two new concepts which are StandardRelate and $\mathrm{M}_{1}$ Relate. The new concepts and their properties are represented as follows:

$<!-$ http://www.semanticweb.org/thesis.owl\#M1Relate $\rightarrow>$

<owl:Class rdf:about="http://www.semanticweb.org/thesis.owl\#M1Relate">

<owl:equivalentClass> 


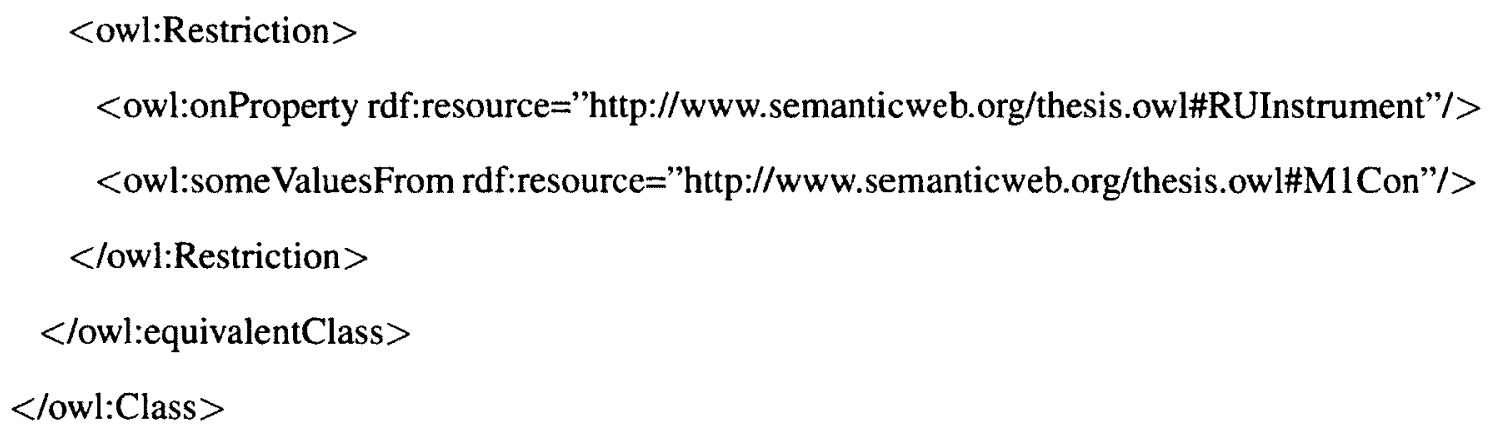

The above representations describe that the concept MIRelate is equivalent to those instances of the domain of the role RUInstrument when the range of the role RUInstrument is restricted to the concept M1Con.

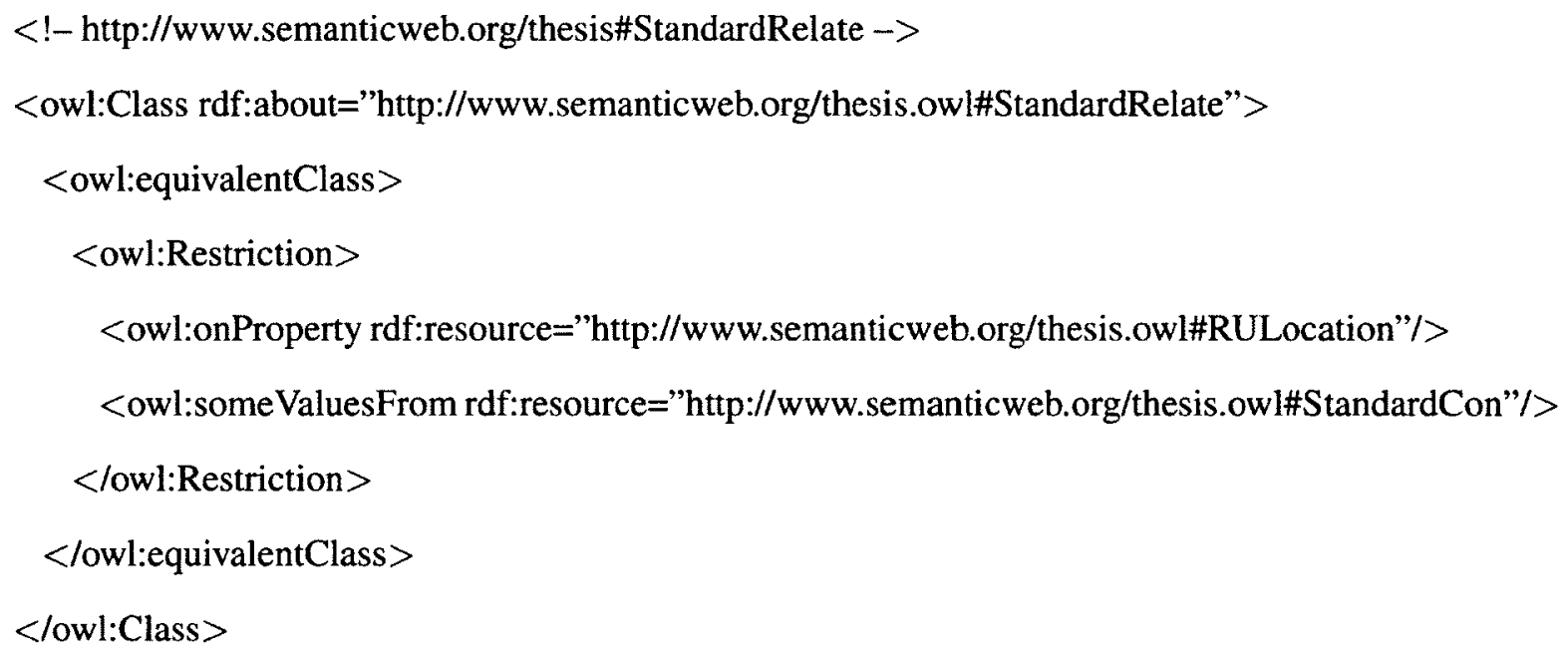

The above representation describes that the concept StandardRelate is equivalent to those instances of the domain of the role RULocation when the range of the role RULocation is restricted to the concept StandardCon.

The object property UnitIns and its inverse which are used for representing the Contextual Guideline 2 (see Page 57 for details), are introduced by the followings:

$<$ !- http://www.semanticweb.org/thesis.owl\#UnitIns $->$ <owl:ObjectProperty rdf:about="http://www.semanticweb.org/thesis.owl\#UnitIns"> 


$$
\begin{aligned}
& \text { <rdfs:range rdf:resource="http://www.semanticweb.org/thesis.owl\#Brand"/> } \\
& \text { <rdfs:domain rdf:resource="http://www.semanticweb.org/thesis.owl\#Care-Unit"/> } \\
& \text { <rdfs:range rdf:resource="http://www.semanticweb.org/thesis.owl\#Manufacturer"/> } \\
& \text { <rdfs:range rdf:resource="http://www.semanticweb.org/thesis.owl\#Model"/> } \\
& \text { <rdfs:domain rdf:resource="http://www.semanticweb.org/thesis.owl\#Nurse"/> } \\
& \text { <rdfs:domain rdf:resource="http://www.semanticweb.org/thesis.owl\#Ward"/> } \\
& \text { </owl:ObjectProperty> }
\end{aligned}
$$

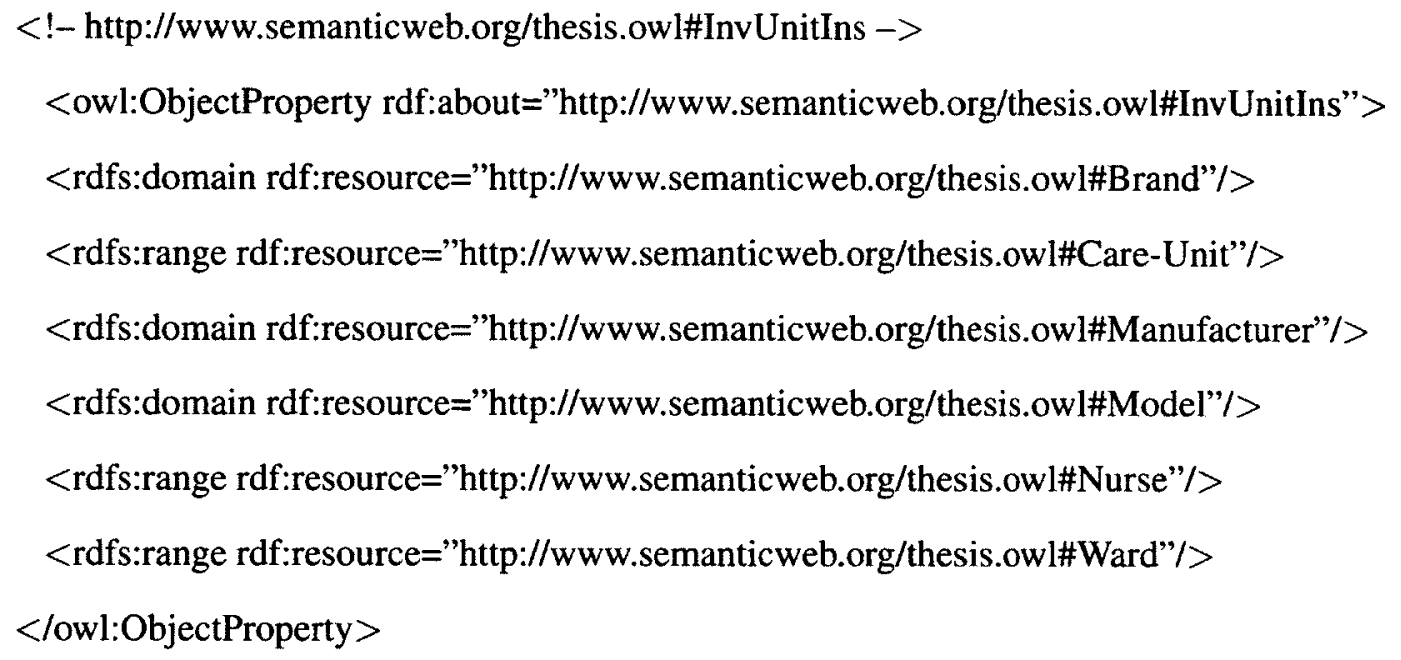

An axiom for representing the Contextual Guideline 2 is introduced in Example 5.2 (Page 57) and is represented in OWL as:

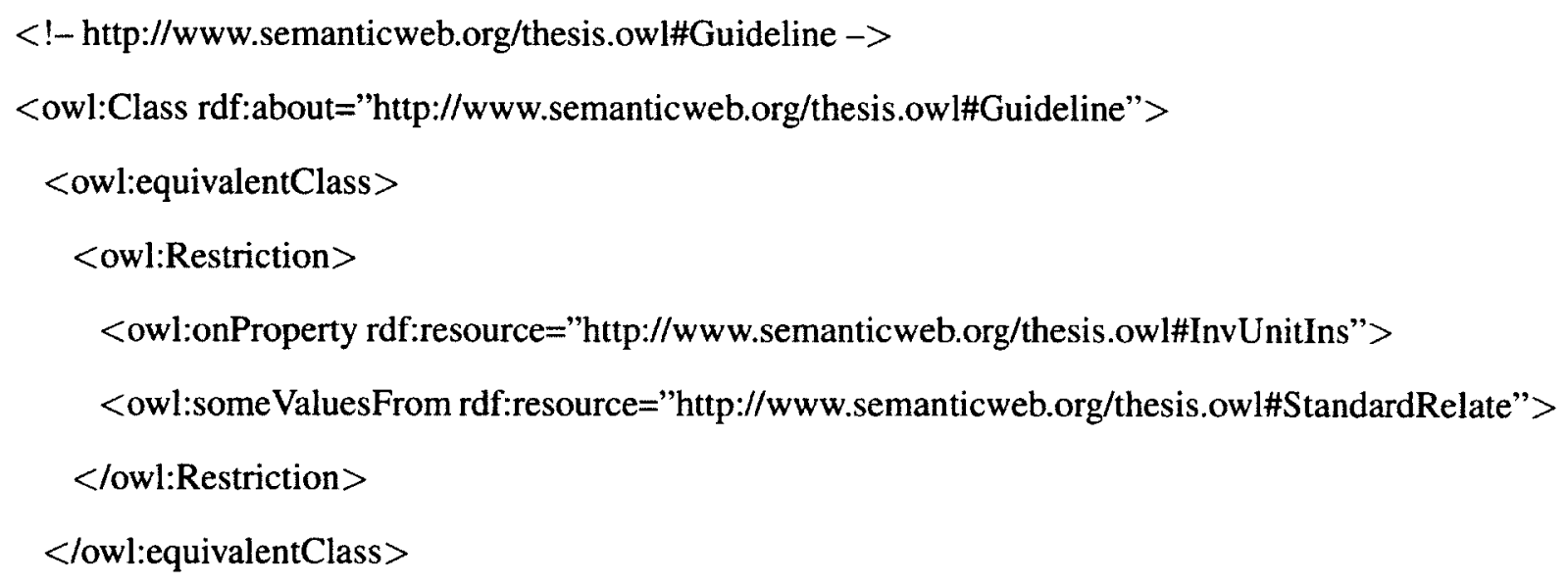


<rdfs:subClassOf rdf:resource="http://www.semanticweb.org/thesis.owl\#M1Relate"/> $<$ lowl:Class $>$

The above representation states that if a medical test is performed in one of the locations related to the standard care unit, the instrument which is used in the test, is made by manufacturer $M_{1}$.

\subsection{ABox Assertions}

In the pervious section, we discussed the representation of the TBox's concepts and roles in OWL. The instances of the concepts and roles in the TBox are asserted in the ABox.

In this experiment, a dimension instance for the dimension Location is stored in a relation Location in the data source. The data source is a PostgreSQL database [51] and the reasoner uses the following information to connect to it:

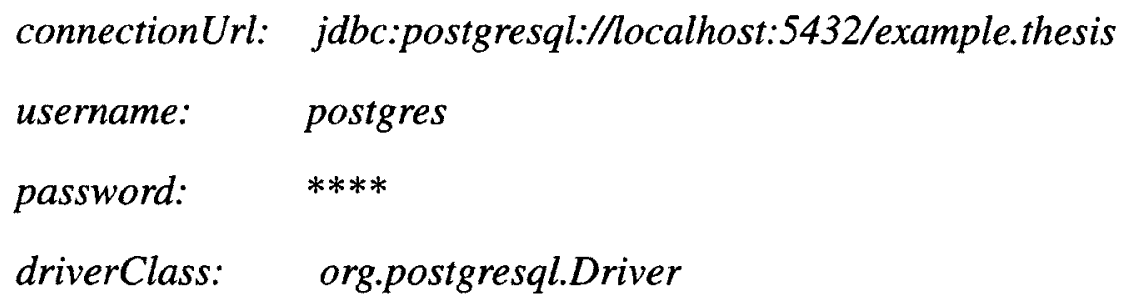

The relation Location in the database example.thesis has the following schema:

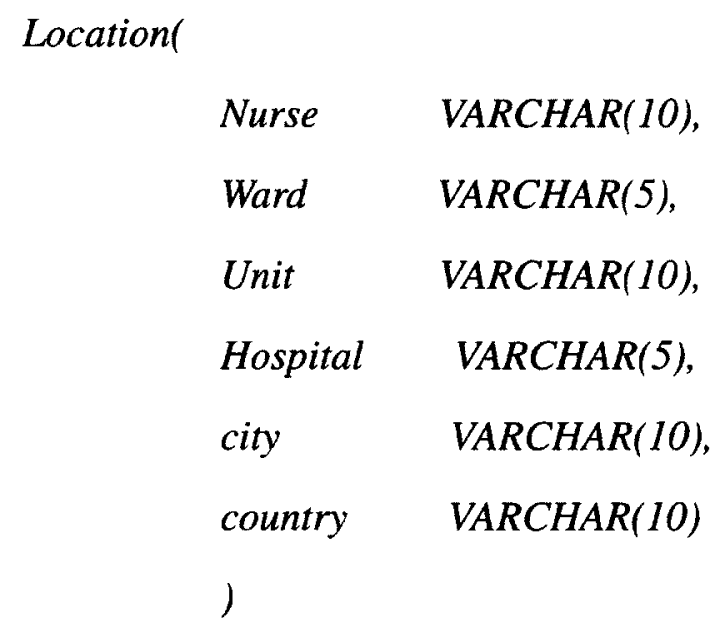


A dimension instance for the contextual dimension Instrument is stored in the relation Instrument in the database. The relation Instrument has the following schema:

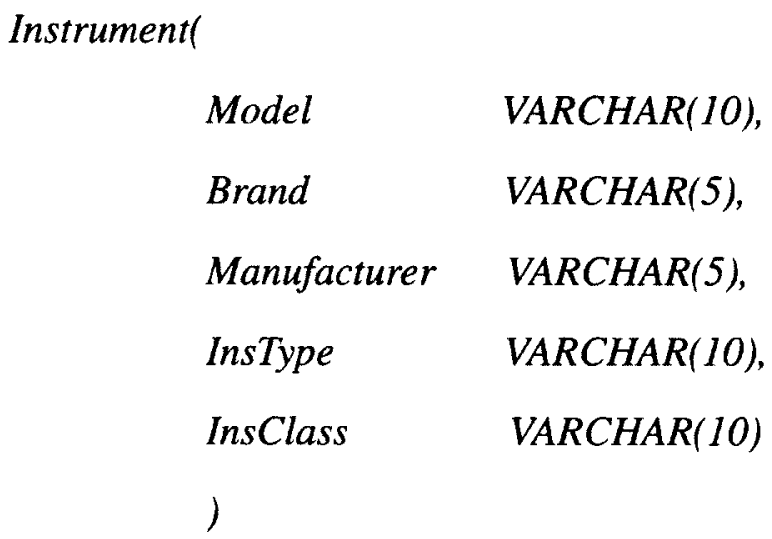

A dimension instance for the contextual dimension Time is stored in the database in the relation Time with the following schema:

$\begin{array}{ll}\text { Timel } & \\ \text { Hour } & \operatorname{VARCHAR}(10), \\ \text { Day } & \operatorname{VARCHAR}(10), \\ \text { Month } & \operatorname{VARCHAR}(10), \\ \text { Shift } & \operatorname{VARCHAR}(10), \\ \text { Week } & \operatorname{VARCHAR}(10), \\ \text { Year } & \text { INT } \\ \text { ) } & \end{array}$

In Section 2.5.1 (Page 30), we discussed the mapping mechanism for linking the existing data in the data source to the ontology. The idea of the representation of the mappings is that by evaluating a query over the data sources, facts for constituting the $\mathrm{ABox} \mathcal{A}$ assertions are retrieved. Figure 12 illustrates this idea.

In this experiment, each mapping is defined as a pair of source, an arbitrary SQL query over the database, and the target, a triple template that contains placeholders referencing column names mentioned in the source query. 


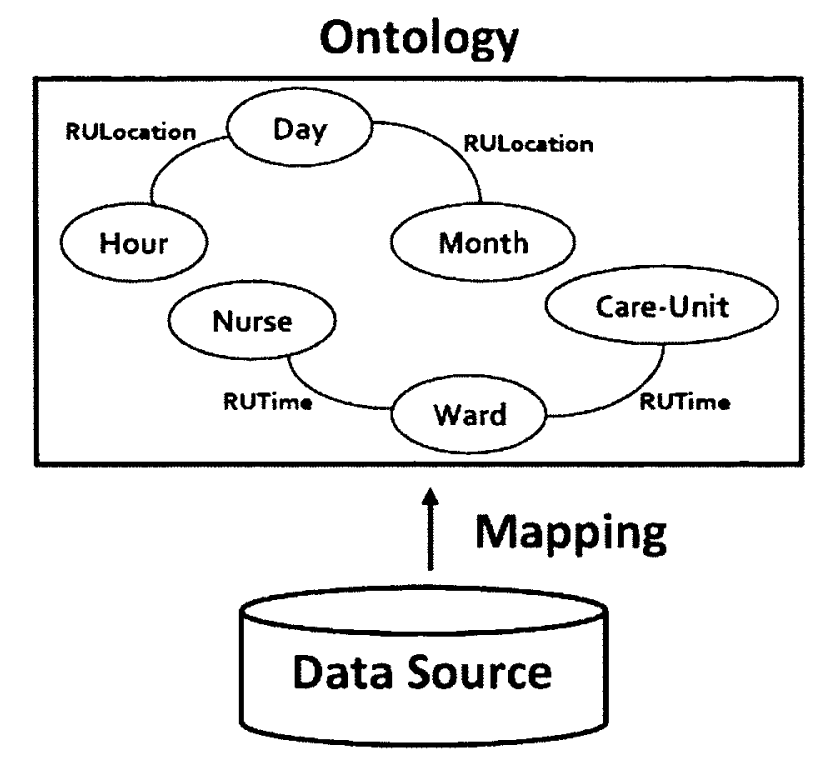

Figure 12: Mapping a Data Source to an Ontology

The query over the concepts and roles in the TBox is called target. The target has a triple template and is written as an RDF subject-predicate-object graph. The Turtle syntax [11] is adapted to represent the target in mapping assertions.

Consider the location relation and its attribute ward in the database. We want to model the fact that the ward $\in$ location corresponds to the Ward of a hospital in the ontology. In order to assert a mapping, the source query is written in SQL and is expressed as follows:

select ward from location

The above query returns the values of the ward attribute of the location dimension. The target query over the concept Ward in the TBox is expressed as:

<"http://www.semanticweb.org/thesis.owl\#Ward-\$ward">a

$<$ http://www.semanticweb.org/thesis.owl\#Ward $>$.

Above, <"http://www.semanticweb.org/thesis.owl\#Ward-\$ward"> is the subject in the RDF triple. $a$ stands for the predicate rdf:type. The URI resource 
<http://www.semanticweb.org/thesis.owl\#Ward> is the object of the RDF triple. A mapping axiom generates RDF triples, one set of RDF triples for each result row returned by the source query. The triples are created by replacing the $\$$ ward in the target with the values from the row.

In this experiment, the relation Location in the database has 10 tuples, and the relation Instrument contains 10 tuples. The relation Time has 20 tuples. Each of the relations HospitalDescription and HospitalFounded has 5 tuples. The relation PatientValueWard contains 10 tuples. After generating the instances of the concepts and roles from the mappings, there exists 120 assertions to the concepts and 164 assertions to the roles in the $\mathrm{ABox}$.

\subsection{Data Quality Assessment}

In this experiment, data quality is addressed in relation to the discrepancy between the actual stored values and the real values that were supposed or expected to be stored, and is determined by a context that enables data quality analysis. Let us now, review the data quality problem that we were deal with in Example 1.4 (Page 7).

Tom is a patient in a hospital. Several times a day different medical tests are performed on him, and his results are recorded. His doctor, John, wants to see Tom's test values every day, to follow his evolution.

The data that John needs about Tom appear, among other, in the PatientValue relation in Table 7.1. John has a quality concern. He asks nurses to perform all the medical tests with instruments made by manufacturer $M_{1}$. 


\begin{tabular}{|c|c|c|}
\hline \multicolumn{3}{|c|}{ Table 7.1: Patient Value } \\
\hline Patient & Value & Time \\
\hline Tom Waits & 38.5 & $11: 45 / 5 / \mathrm{Sep} / 2011$ \\
Tom Waits & 38.2 & $12: 10 / 5 / \mathrm{Sep} / 2011$ \\
Tom Waits & 38.1 & $11: 50 / 6 / \mathrm{Sep} / 2011$ \\
Tom Waits & 38.0 & $12: 15 / 7 / \mathrm{Sep} / 2011$ \\
Tom Waits & $110 / 70$ & $11: 45 / 8 / \mathrm{Sep} / 2011$ \\
Lou Reed & 37.9 & $12: 10 / 5 / \mathrm{Sep} / 2011$ \\
\hline
\end{tabular}

The context has information about hospital guideline:

Contextual Hospital Guideline 2: 'Medical tests on patients in standard care unit has to be taken with instruments made by manufacturer $M_{1}{ }^{\prime}$ (Page 8 ).

Consider the contextual relation PatientValueWard in Table 4.1 (Page 51). It contains all the values of different medical tests that are performed on patients by using instruments in different wards of the hospital at different times. The information explicitly provided by the contextual relation PatientValueWard is about the Ward category, but data about the Care Units, that could be used in combination with Guideline 2, belongs to a higher or more general category in the Location dimension (Figure 3, Page 9).

Contextual relation PatientValueWard is translated into the DL-ontology in Example 4.6 (Page 51) and is represented in OWL on Page 71. In addition, Contextual Hospital Guideline 2 is translated into the axioms in the TBox in Example 5.2 (Page 57) and is represented in OWL on Page 73. The contextual dimension Location (Figure 3, Page 9) that could be used in combination with Guideline 2 is translated into the DL-ontology in Examples 4.1 to 4.5, and its OWL representation is discussed on Pages 67 to 71.

The axioms for the representation of the Contextual Hospital Guideline 2 hold in all of 


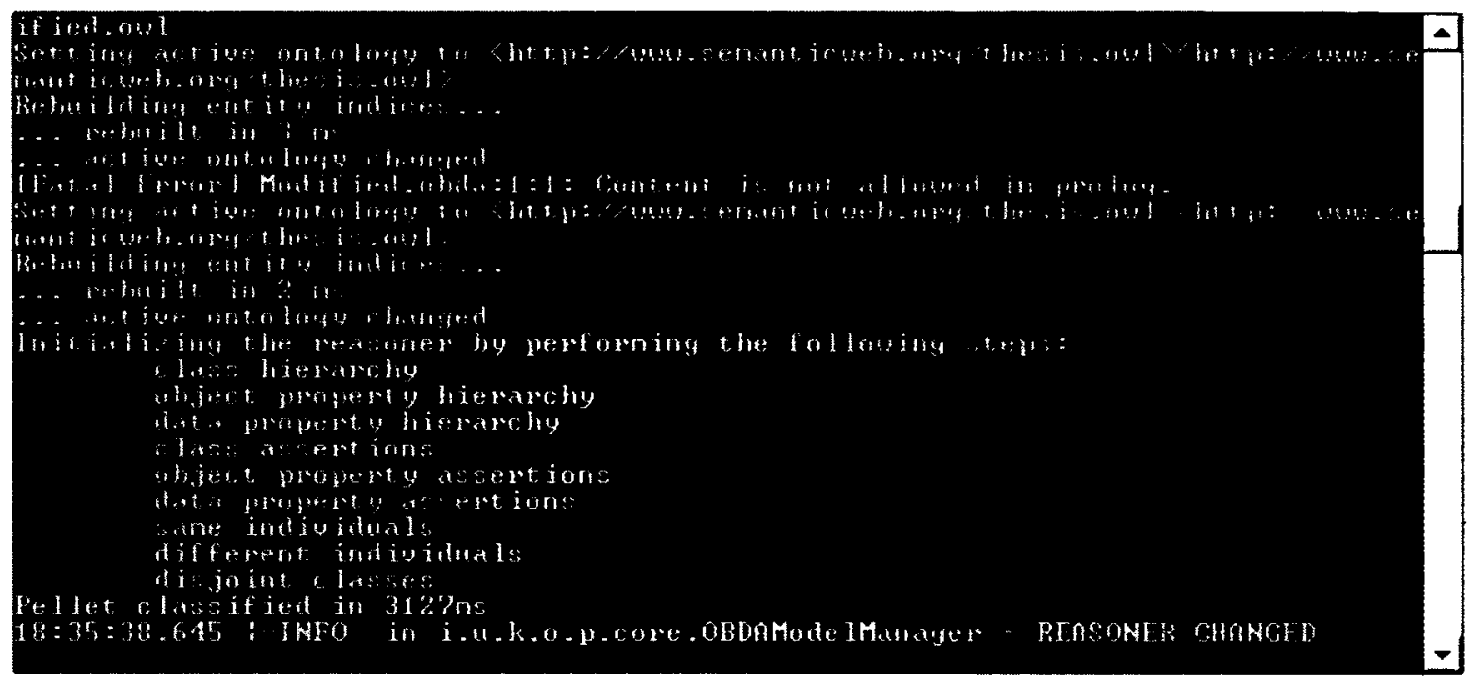

Figure 13: Ontology Satisfiability Verification

the models of the ontology if the ontology is satisfiable. The satisfiability of the represented ontology in this experiment is verified by the Pellet reasoner [54], which is an open-source Java based OWL DL reasoner. The log file of the reasoning process is illustrated in Figure 13.

The Contextual Hospital Guideline 2 provides the information about the care units of the hospital. The care unit that each ward belongs to, can be realized by rolling up from the Ward category to the Care-Unit category in the contextual dimension Location (Figure 3, Page 9). The navigation from the Ward category to the Care-Unit category in the ontological representation of the contextual dimension Location is a reasoning service that is provided by the reasoner. Having the ontological representation of the contextual relation PatientValueWard (Table 4.1, Page 51), and the ontological representation of the Contextual Hospital Guideline 2, the following query asks about those tests' results of the patients that are taken in the standard care-unit. The query is written in terms of the concepts and roles 
of the ontology, and is expressed as the conjunctive queries, in particular Datalog [21].

$$
\begin{gathered}
\mathcal{Q}(p, v, h) \leftarrow \quad P W_{\text {Patient }}(p w, p), P W_{\text {Value }}(p w, v), P W_{\text {Hour }}(p w, h), \\
P W_{\text {Ward }}(p w, w), \text { StandardRelate }(w),
\end{gathered}
$$

where the roles $P W_{\text {Patient }}, P W_{\text {Hour }}, P W_{\text {Value }}$ and $P W_{W \text { ard }}$ are the roles of the ontology which are introduced for the translation of the contextual relation PatientValueWard (Table 4.1, Page 51) into the TBox, StandardRelate is the concept of the ontology which contains all the instances of the Location dimension that has the element standard as an ancestor in the Care-Unit category (previously introduced for the representation of the Hospital Guideline 2 into the ontology in Example 5.2, Page 57). In our experiment this query is expressed in SPARQL [49] as:

PREFIX rdf: <http://www.w3.org/1999/02/22-rdf-syntax-ns\#>

PREFIX foaf: <http://www.semanticweb.org/thesis.owl\#>

SELECT DISTINCT ?patient ?value ?hour

WHERE $\{$ ?pw foaf:PWPatient ?patient. ?pw foaf:PWValue ?value.

?pw foaf:PWHour ?hour. ?pw foaf:PWWard ?w. ?w rdf:type foaf:StandardRelate. $\}$

where the roles PWPatient, PWValue, PWWard and PWHour stand for the owl representation of the roles PWPatient, PWValue, PWWard and PWHour of the DL-ontology respectively, and the concept StandardRelate corresponds to the owl representation of the concept StandardRelate in the DL-ontology.

The above query is asking about the test results of patients that are taken in the locations which have Standard as an ancestor in the Care-Unit category at different times. The concept StandardRelate is equivalent to the domain of the role RULocation when the range of the role RULocation, is restricted to the concept StandardCon (the concept that contains the element Standard, see Example 5.2, Page 57 for details). The set of axioms of the 
ontological representation of the Hospital Guideline 2 declare that the instruments that are used in one of locations related to the concept StandardRelate are made by the manufacturer $M_{1}$. The satisfiability of these axioms is verified by the Pellet reasoner and the reasoner by navigating through the hierarchy obtains all the locations that have the element Standard as an ancestor in the Care-Unit category. Those values of the tests' results that are performed in the wards belong to the StandardRelate category are returned by the reasoner as the certain answers to the query $\mathcal{Q}$. The certain answers to the query $\mathcal{Q}$ (quality instance) is illustrated in Table 7.2.

\begin{tabular}{|c|c|l|}
\hline \multicolumn{2}{|c|}{ Table 7.2: Certain Answers } \\
\hline Patient & Value & \multicolumn{1}{c|}{ Hour } \\
\hline Patient-Tom Waits & Value-38.5 & Hour-11:45/5/Sep/2011 \\
Patient-Tom Waits & Value-38.2 & Hour-12:10/5/Sep/2011 \\
Patient-Tom Waits & Value-38.1 & Hour-11:50/6/Sep/2011 \\
Patient-Tom Waits & Value-38.0 & Hour-12:15/7/Sep/2011 \\
Patient-Lou Reed & Value-37.9 & Hour-12:10/5/Sep/2011 \\
\hline
\end{tabular}

The answers to the query $\mathcal{Q}$ is intended to contain only results of the tests which are performed by the instruments that are made by manufacturer $M_{1}$.

Every model of the ontology provides a set of answers to the query $\mathcal{Q}$. Each set of answers (also called the legal contextual instance (LCI) for the ontological context) also contains the interpretation of the certain answers and belongs to the class of the quality versions of the relation PatientValue (Table 1.1, Page 3). The certain answers appear in Table 7.1 corresponds to the smallest LCI for the ontological context, no subset of it is an LCI. 
In this experiment, the several possible contextual instance may become admissible candidates to be used for data quality assessment. The quality of relation PatientValue (Table 7.1) is assessed using the quality measure $q m_{1}$ which is introduced in [16] (See Section 2.2, Page 21 for details):

$$
q m_{1}(D):=\frac{|D|-\max \left\{\left|D_{\mathcal{P}}^{\prime}(I)\right|: I \text { is LCI }\right\}}{|D|}
$$

The number of tuples of the relation Patient Value which is under quality assessment $(|D|)$ is 6 and the number of the certain answers to the query $\mathcal{Q}\left(\left|D_{\mathcal{P}}^{\prime}(I)\right|\right)$ is 5 . The quality measure $q m_{1}$ is evaluated to 0.17 which means the data in the relation PatientValue are almost quality data (since the value is near to 0 ). 


\section{Chapter 7}

\section{Conclusions and Future Work}

\subsection{Conclusions}

A hierarchal framework for organizing data quality dimensions is proposed in [56], with a discussion of high-quality data as intrinsically good, contextually appropriate for the task, and clearly represented and accessible to the data consumer. Research on data quality problems are mainly based on the implicit assumption that data errors are mostly syntactic errors [10]. As discussed in [38], data quality may also be related to the semantic of data. For example, a data quality problem occurs when there is a difference between the intended meaning (according to its producer) and interpreted meaning (according to its consumer) of a data value. The discrepancy occurs because the communication between the data producer and costumer is not clear.

In this work, we discussed about the dependency of the quality of data on contexts. There are proposals on using contexts in data management before [16] [19] [45].

There are some previous proposals for using contexts in data management [16] [18] [19], including some dimensional aspects [20]. In [18], contextual commonsense assumptions are expressed as sets of variables that may be a point of interest for an agent. The values of those variables influence the agent's actions. The representation of context in [18] [20] is based on a tree-like context model, whose instances are sets of context 
elements, in essence, collections of attribute-value pairs, e.g. role $=$ ' $C E O$ ', situation $=$ 'in-office' and location = 'city'. Context-aware views are automatically or semi automatically generated from a given database and a contextual instance, allowing to see the data from different perspectives or points of view. The model also allows for the specification of constraints on a context instance, e.g. stating that when a role is 'CEO', a situation cannot be 'on-site'. In our work, this type of conditions is captured by interdimensional constraints.

A context can be defined as a partial description of the world [32] [33]. Each context is represented in a formal language, and checking the satisfiability of a formula in that language is local and performed in its context. In addition, contexts mutually influence themselves and different forms of reasoning, in different contexts, are made compatible.

The other proposals for context-aware systems is discussed in both [43] [44]. The model that is argued in [43] is the natural extension of the relational model in which contexts are the first class citizens. The context is represented from different perspectives in different levels of detail. In [43] [44] an extension of relational algebra is introduced for querying multidimensional context.

Our framework for data quality assessment is based on [15] [16]. In [16], quality instances are obtained by interaction with additional contextual data or metadata. The contexts in [16] are represented in the theory of relational databases and are defined as additional information that exist in some relations.

Based on the data quality assessment framework [15] [16], the quality of a database $D$ is assessed by considering a context which is represented as an external system containing additional data, metadata, and definitions of quality predicates. The instance $D$ is 'put in context' through the schema mappings; and after contextual processing of the data, a collection of alternative clean versions $D^{\prime}$ of $D$ is produced. The quality of $D$ is measured in terms of its distance to $D^{\prime}$.

In this work we extend the context model introduced in [15] [16], by adding rules, 
dimensions, dimensional data, and, finally putting all this together as a DL ontology. An ontology provides language components and constructs for representing knowledge about a domain of interest, and also reasoning capabilities.

There are other proposals for representing data warehouse conceptual schema [28] [29] [52]. They start from the descriptions of DWH schemas as extended entity relationship (EER) diagrams, and provide the formal semantics for the EER representation via the $\mathcal{A L C F I}$ description logic [35]. The proposed data model is used to introduce complex descriptions for the structure of the aggregated entities. The high expressive DL, $\mathcal{A L C F I}$ is used to provide the semantic to the proposed EER including aggregated entities. $\mathcal{A L C F} \mathcal{I}$ description logic offers role composition and concept disjunction for representing aggregated concepts. However, using a multidimensional model and a multidimensional databases within the context serves purposes not traditionally found in data warehousing. Hence, in the proposed ontological representation of context, there is no need for representing aggregated entities. The complexity of $\mathcal{A L C F} \mathcal{I}$ is PSpace-complete for concept satisfiability checking [35].

In this work, we have focused mainly on the introduction of contextual dimension for navigating in search for the data required to assess the quality of another set of data. This also allows doing data quality assessment from multiple views and level of granularity. Furthermore, we also introduced the intra- and interdimensional semantic constraints. These constraints are particularly interesting since they do not commonly appear in MDDBs. We also show how these constraints can be captured as axioms in the DL ontology.

In this thesis, we also discussed about the representation of the $\mathcal{M D}$ constraint, homogeneity. Notice that for the representation of the $\mathcal{M D}$ constraint, strictness, the combination of role transitivity and functionality is problematic and result in the undecidability [36]. For example, consider the Instrument dimension in Example 4.5. For expressing that each type of the instrument from the Type category can have only one parent in the Class category, it is not possible to have the axiom such as: Type $\square \geq 2$ Instrument.Class $\sqsubseteq$ perp. 
The previous axiom states that each member of the Type category can have at most one parent in the Class category.

In this work, we represented the context as an ontology written in DL-Lite family of description logic. More precisely, we represented contexts as ontologies written in a de-

scription logic of the $D L$-Lite family [23], actually, $D L$-Lite $\mathrm{Horn}^{(\mathcal{H N})^{+}}$[5]. DL-Lite family of description logic supports most of the reasoning tasks that are done by DLs, while maintaining the good computational properties.

In this thesis, we have focused mainly on the introduction of contextual dimension for navigating in search for the data required to assess the quality of another set of data. This also allows doing data quality assessment from multiple views and level of granularity.

\subsection{Future Work}

Our work leaves several open problems that are matter of ongoing and future research:

- An investigation of the quality query answering through the ontological context.

- An exploration on the alternative representations for the contextual ontologies and the access to data through them, e.g. using Datalog $+[21]$.

- An integration of our framework and the specification of explicit, lower-level data quality predicates [38] should be discussed and defined.

- A detailed and comparative analysis of the quality measures mentioned in this thesis should be investigated. 


\section{List of References}

[1] KAON2 - A Scalable Reasoning Tool for the Semantic Web. In http://kaon2.semanticweb.org/.

[2] A. Acciarri, D. Calvanese, G. De Giacomo, D. Lembo, M. Lenzerini, M. Palmieri, and R. Rosati. QuOnto: Querying Ontologies. In Proceedings of the National Conference on Artificial Intelligence, volume 20, page 1670. Menlo Park, CA; Cambridge, MA; London; AAAI Press; MIT Press; 1999, 2005. Download from http://ontop.inf.unibz.it/?page_id $=11$

[3] D. Allemang and J. Hendler. Semantic Web for the Working Ontologist: Effective Modeling in RDFS and OWL. Morgan Kaufmann, 2011.

[4] M. Arenas, P. Barceló, L. Libkin, and F. Murlak. Relational and XML Data Exchange. Synthesis Lectures on Data Management. Morgan \& Claypool Publishers, 2010.

[5] A. Artale, D. Calvanese, R. Kontchakov, and M. Zakharyaschev. The DL-Lite Family and Relations. Journal of Artificial Intelligence Research (JAIR), 36:1-69, 2009.

[6] P. Atzeni, S. Ceri, S. Paraboschi, and R. Torlone. Database Systems - Concepts, Languages and Architectures. McGraw-Hill Book Company, 1999.

[7] F. Baader, I. Horrocks, and U. Sattler. Description Logics as Ontology Languages for the Semantic Web. Mechanizing Mathematical Reasoning, pages 228-248, 2005.

[8] F. Baader and W. Nutt. Basic Description Logics. The Description Logic Handbook: Theory, Implementation, and Applications, pages 43-95. Cambridge University Press, 2003.

[9] C. Batini, S. Ceri, and S. B. Navathe. Conceptual Database Design: An EntityRelationship Approach. Benjamin/Cummings, 1992.

[10] C. Batini and M. Scannapieco. Data Quality: Concepts, Methodologies and Techniques. Data-Centric Systems and Applications. Springer, 2006. 
[11] D. Beckett and T. Berners-Lee. Turtle-Terse RDF Triple Language. January 2004. W3C Team Submission: http://www.w3.org/TeamSubmission/turtle/ (last retrieved January 2013)

[12] P. A. Bernstein and S. Melnik. Model Management 2.0: Manipulating Richer Mappings. In Proceedings of the 2007 ACM SIGMOD International Conference on Management of Data, SIGMOD '07, pages 1-12, New York, NY, USA, 2007. ACM.

[13] L. Bertossi and L. Bravo. Consistent Query Answers in Virtual Data Integration Systems. In Inconsistency Tolerance, pages 42-83. Springer LNCS 3300, 2004.

[14] L. Bertossi, L. Bravo, and M. Caniupán. Consistent Query Answering in Data Warehouses. In Alberto Mendelzon International Workshop of Foundations of Data Management (AMW' 09). CEUR-WS, volume 450, page $12 \mathrm{pp}, 2009$.

[15] L. Bertossi and F. Rizzolo. Contexts and Data Quality Assessment. In Journal submission, 2012.

[16] L. Bertossi, F. Rizzolo, and L. Jiang. Data Quality Is Context Dependent. In Workshop on Enabling Real-Time Business Intelligence (BIRTE 2010). Collocated with VLDB 2010., pages 52-67. Springer LNBIP 48, 2010.

[17] L. Bertossi and J. Chomicki. Query Answering in Inconsistent Databases. Logics for Emerging Applications of Databases, pages 43 - 83, 2003.

[18] C. Bolchini, C. Curino, G. Orsi, E. Quintarelli, R. Rossato, F. A. Schreiber, and L. Tanca. And What Can Context Do for Data? Communications of the ACM, 52(11):136-140, 2009.

[19] C. Bolchini, C. Curino, E. Quintarelli, F. A. Schreiber, and L. Tanca. A Data-Oriented Survey of Context Models. SIGMOD Record, 36(4):19-26, 2007.

[20] C. Bolchini, C. Curino, E. Quintarelli, F. A. Schreiber, and L. Tanca. Context Information for Knowledge Reshaping. International Journal of Web Engineering and Technology., 5(1):88-103, 2009.

[21] A. Calì, G. Gottlob, and T. Lukasiewicz. Datalog Extensions for Tractable Query Answering over Ontologies. In Semantic Web Information Management, pages 249279. Springer, 2010.

[22] D. Calvanese, G. De Giacomo, D. Lembo, M. Lenzerini, A. Poggi, and R. Rosati. Ontology-Based Database Access. In Proceedings of the 15th Italian Conference on Database Systems (SEBD 2007), pages 324-331, 2007. 
[23] D. Calvanese, G. D. Giacomo, D. Lembo, M. Lenzerini, and R. Rosati. Tractable Reasoning and Efficient Query Answering in Description Logics: The DL-Lite Family. Journal of Automated Reasoning, 39(3):385-429, 2007.

[24] D. Calvanese, G. D. Giacomo, D. Lembo, M. Lenzerini, R. Rosati, and M. Ruzzi. Using OWL in Data Integration. Semantic Web Information Management, pages $397-$ 424. Springer, 2010.

[25] D. Calvanese, D. G. Giuseppe, D. Lembo, M. Lenzerini, A. Poggi, M. RodriguezMuro, and R. Rosati. Ontologies and Databases: The DL-Lite Approach. Semantic Technologies for Informations Systems - 5th Int. Reasoning Web Summer School (RW 2009), volume 5689 of Lecture Notes in Computer Science, pages 255-356. Springer, 2009.

[26] P. P. Chen. The Entity-Relationship Model Toward a Unified View of Data. ACM Transactions on Database Systems, 1(1):9-36, Mar. 1976.

[27] J. Coutaz, J. L. Crowley, S. Dobson, and D. Garlan. Context is Key. Communications of the ACM, 48(3):49-53, 2005.

[28] E. Franconi and U. Sattler. A Data Warehouse Conceptual Data Model for Multidimensional Aggregation. In Workshop on Design and Management of Data Warehouses (DMDW'99), volume 19, page 13, 1999.

[29] E. Franconi and U. Sattler. A Data Warehouse Conceptual Data Model for Multidimensional Aggregation: A Preliminary Report. Italian Association for Artificial Intelligence AI*IA Notizie, 1:9-21, 1999.

[30] B. Glimm, I. Horrocks, C. Lutz, and U. Sattler. Conjunctive Query Answering for the Description Logic SHIQ. In Proceedings of the International Joint Conferences on Artificial Intelligence, pages 399-404, 2007.

[31] T. Gruber. A Translation Approach to Portable Ontology Specifications. Knowledge Acquisition, 5(2):199-220, 1993.

[32] Giunchiglia, F. Contextual Reasoning. In Proceeding of International Joint Conference on Artificial Intelligence on Using Knowledge in its Context, 1993, pp. 39-49.

[33] Ghidini, C. and Giunchiglia, F. Local Models Semantics, or Contextual Reasoning = Locality + Compatibility. Artificial Intelligence, , 2001, 127:221-259.

[34] V. Haarslev and R. Müller. Racer System Description. Automated Reasoning, pages 701-705, 2001. 
[35] I. Horrocks and U. Sattler. A Description Logic with Transitive and Inverse Roles and Role Hierarchies. Journal of Logic and Computation, 9(3):385-410, 1999.

[36] I. Horrocks, U. Sattler, and S. Tobies. Practical Reasoning for Very Expressive Description Logics. Proceedings of the 6th International Conference on Logic Programming and Automated Reasoning. p.161-180, September 06-10, 1999

[37] C. A. Hurtado, C. Gutiérrez, and A. O. Mendelzon. Capturing Summarizability with Integrity Constraints in OLAP. ACM Transactions on Database Systems, 30(3):854 $886,2005$.

[38] L. Jiang, A. Borgida, and J. Mylopoulos. Towards a Compositional Semantic Account of Data Quality Attributes. In Proceedings of the 27th International Conference on Conceptual Modelling (ER'08), pages 55-68, 2008.

[39] R. Kimball. The Data Warehouse Toolkit: Practical Techniques for Building Dimensional Data Warehouses. John Wiley, 1996.

[40] H. Knublauch, M. Musen, and A. Rector. Editing Description Logic Ontologies with the Protégé OWL Plugin. In International Workshop on Description Logics, volume 49, 2004.

[41] O. Lassila, R. Swick, et al. Resource Description Framework (rdf) Model and Syntax Specification. W3C Recommendation, World Wide Web Consortium, Feb. 1999; www.w3.org/TR/REC-rdf-syntax.

[42] M. Lenzerini. Data Integration: A Theoretical Perspective. In Proceedings of the Twenty-First ACM SIGMOD-SIGACT-SIGART Symposium on Principles of Database Systems, pages 233-246. ACM, 2002.

[43] D. Martinenghi and R. Torlone. Querying Context-Aware Databases. Flexible Query Answering Systems, pages 76-87, 2009.

[44] D. Martinenghi and R. Torlone. Querying Databases with Taxonomies. Conceptual Modeling-ER 2010, pages 377-390, 2010.

[45] J. McCarthy. Notes on Formalizing Context. 1:555-560, 1993.

[46] D. McGuinness, F. Van Harmelen. OWL Web Ontology Language Overview. World Wide Web Consortium (W3C) Recommendation, Feb. 2004; www.w3.org/TR/owlfeatures. 
[47] B. Motik, P. Patel-Schneider, B. Parsia, C. Bock, A. Fokoue, P. Haase, R. Hoekstra, I. Horrocks, A. Ruttenberg, and U. Sattler. OWL 2 Web Ontology Language: Structural Specification and Functional-Style Syntax. W3C Recommendation, 27, 2009.

[48] B. Motik, R. Shearer, and I. Horrocks. Hypertableau Reasoning for Description Logics. Journal of Artificial Intelligence Research, 36:165-228, 2009.

[49] J. Pérez, M. Arenas, and C. Gutierrez. Semantics and Complexity of SPARQL. ACM Transactions on Database Systems (TODS), 34(3):16, 2009.

[50] A. Poggi, D. Lembo, D. Calvanese, G. D. Giacomo, M. Lenzerini, and R. Rosati. Linking Data to Ontologies. Journal of Data Semantics, 10:133-173, 2008.

[51] PostgreSQL Global Development Group. PostgreSQL. http://www.postgresql.org, 2008.

[52] U. Sattler. Description Logics for the Representation of Aggregated Objects. Proceedings of the 14th European Conference on Artificial Intelligence, pages 239-243. IOS Press, Amsterdam, 2000.

[53] R. Shearer, B. Motik, and I. Horrocks. Hermit: A Highly-Efficient OWL Reasoner. In Proceedings of the 5th International Workshop on OWL: Experiences and Directions (OWLED 2008), pages 26-27, 2008.

[54] E. Sirin, B. Parsia, B. Grau, A. Kalyanpur, and Y. Katz. Pellet: A Practical OWLDL Reasoner. Web Semantics: Science, Services and Agents on the World Wide Web, 5(2):51-53, 2007. Download form http://clarkparsia.com/pellet/

[55] M. Vardi. The Complexity of Relational Query Languages. In Proceedings of the Fourteenth Annual ACM Symposium on Theory of Computing, pages 137-146. ACM, 1982.

[56] Wang, R. and Strong, D. Beyond Accuracy: What Data Quality Means to Data Consumers. Journal of Management and Information Systems, , 1996, 12(4):5-33. 


\section{Appendix A}

\section{OWL Representation of the Context}

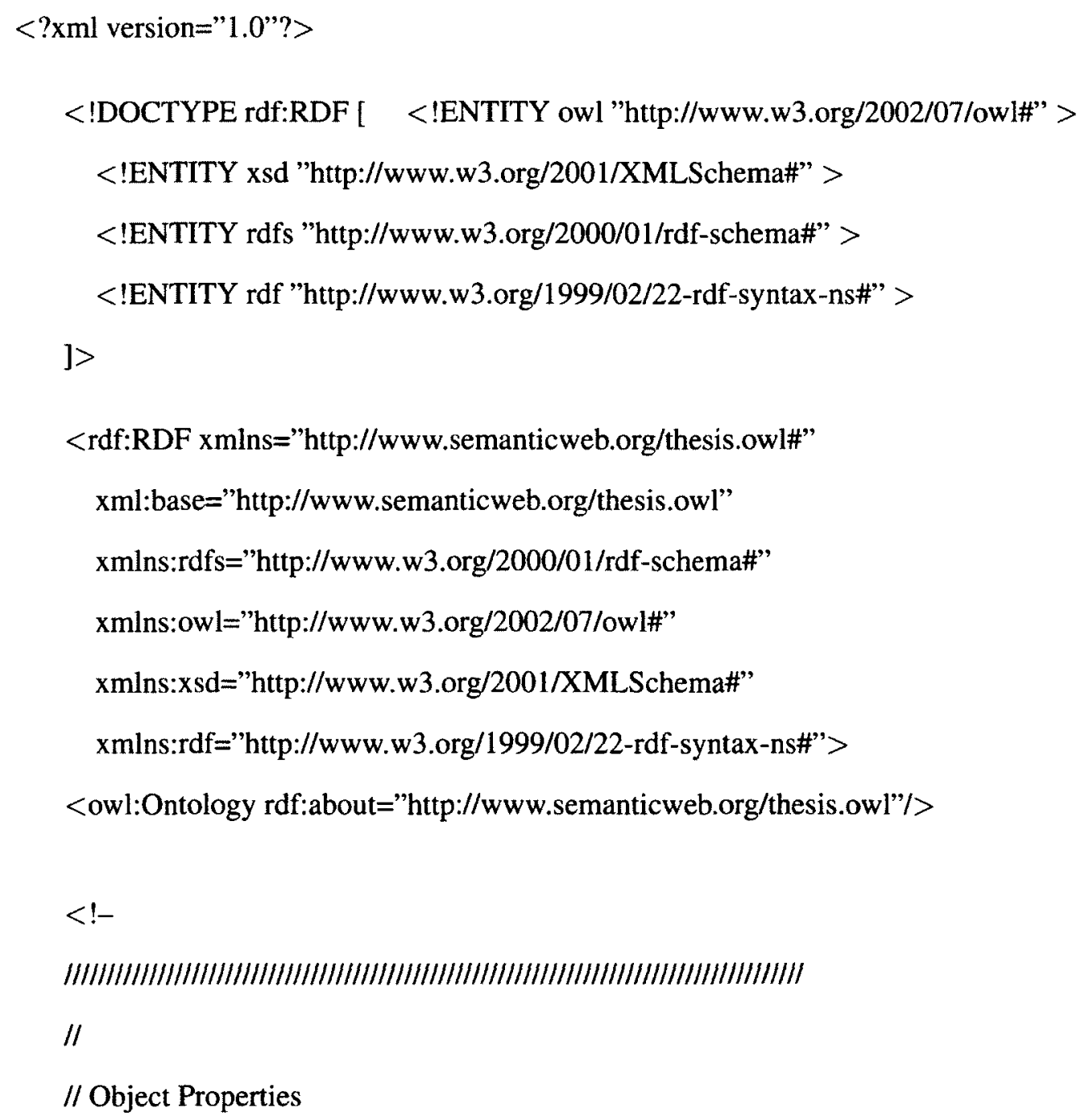




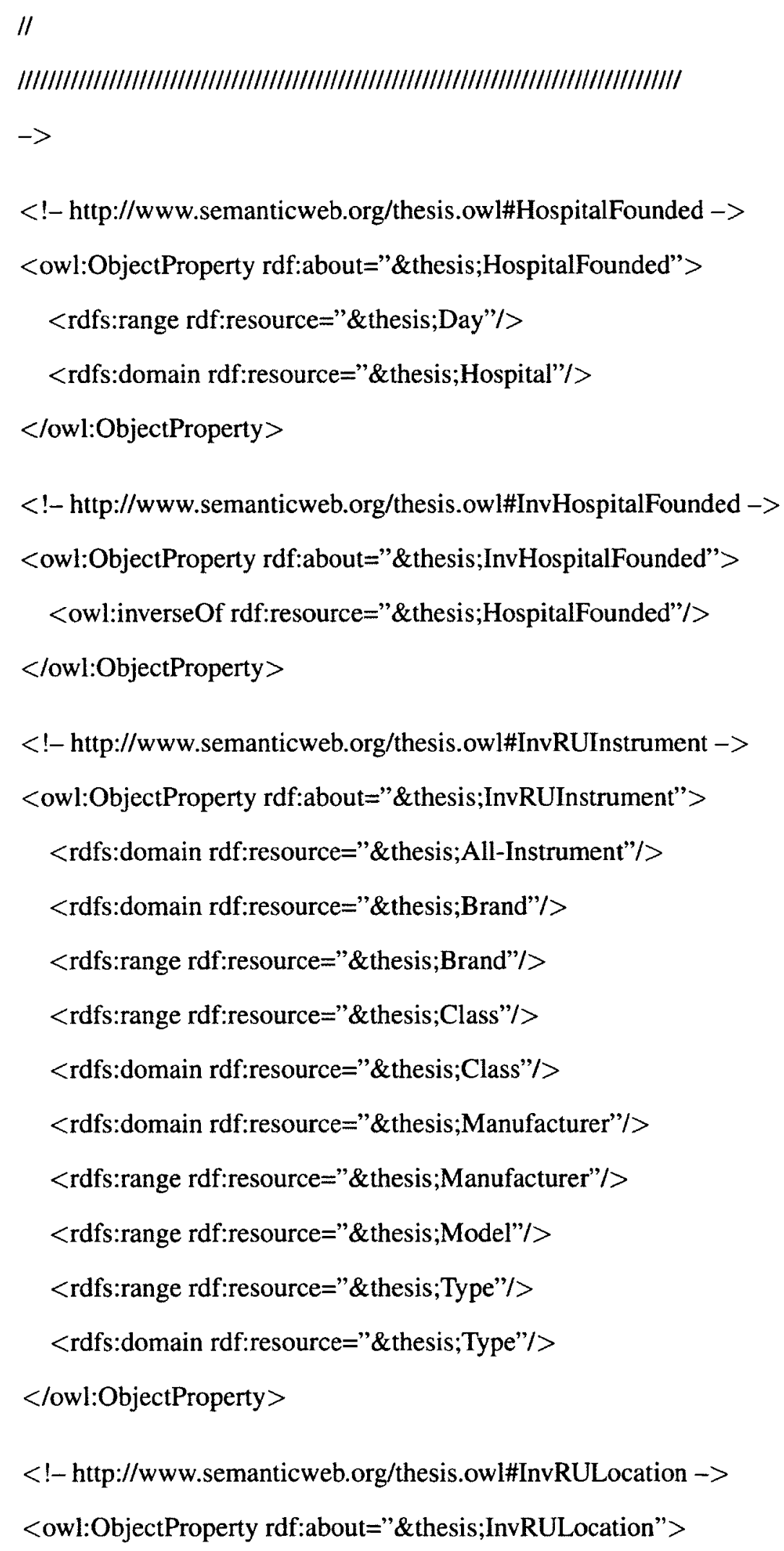




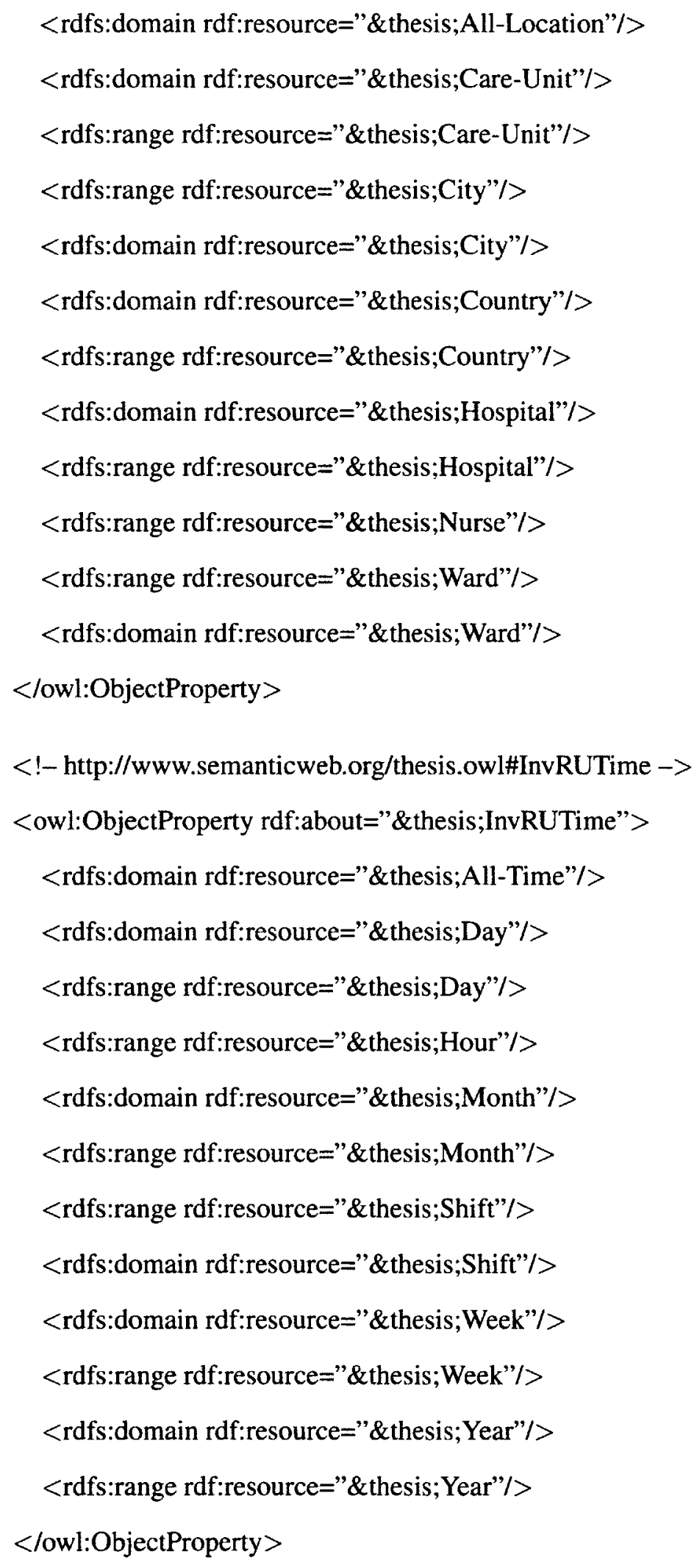




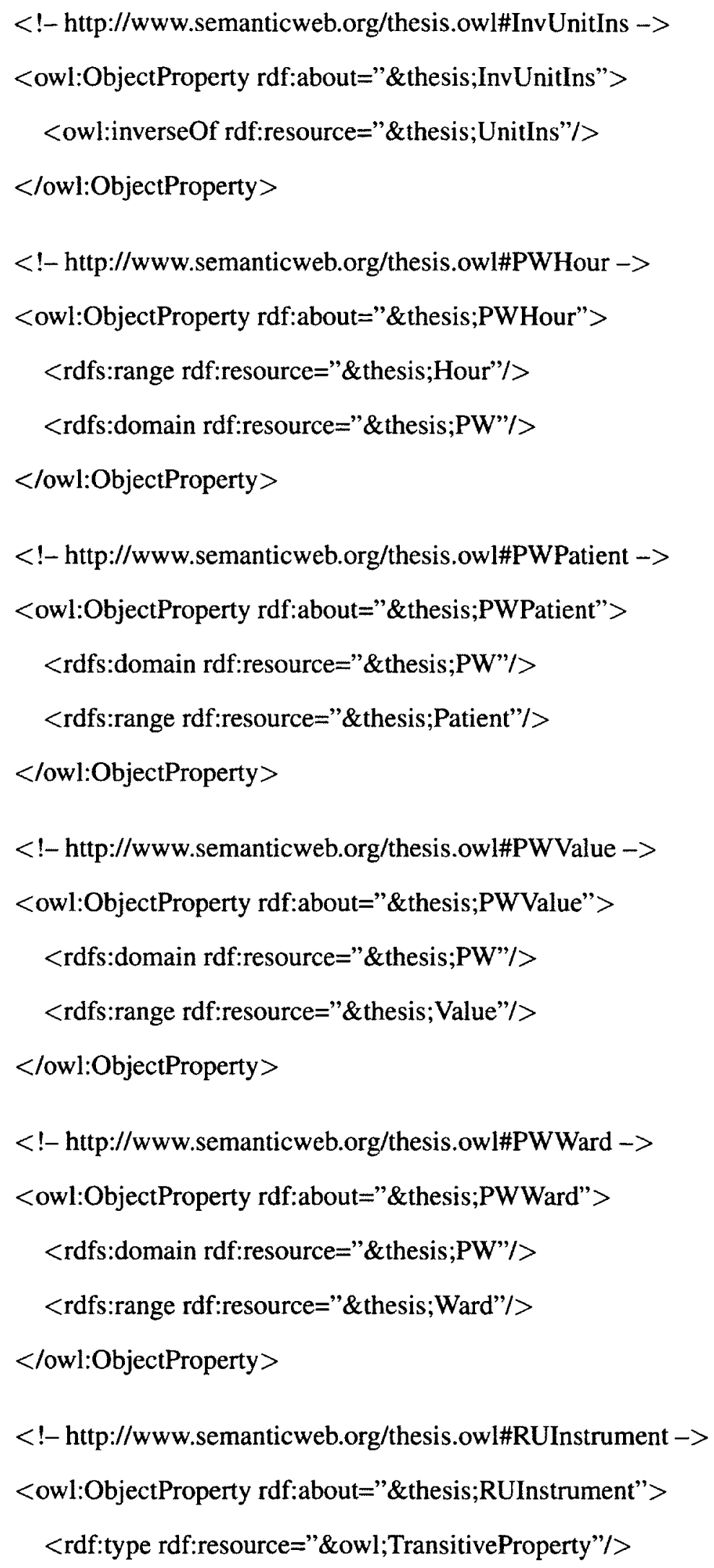




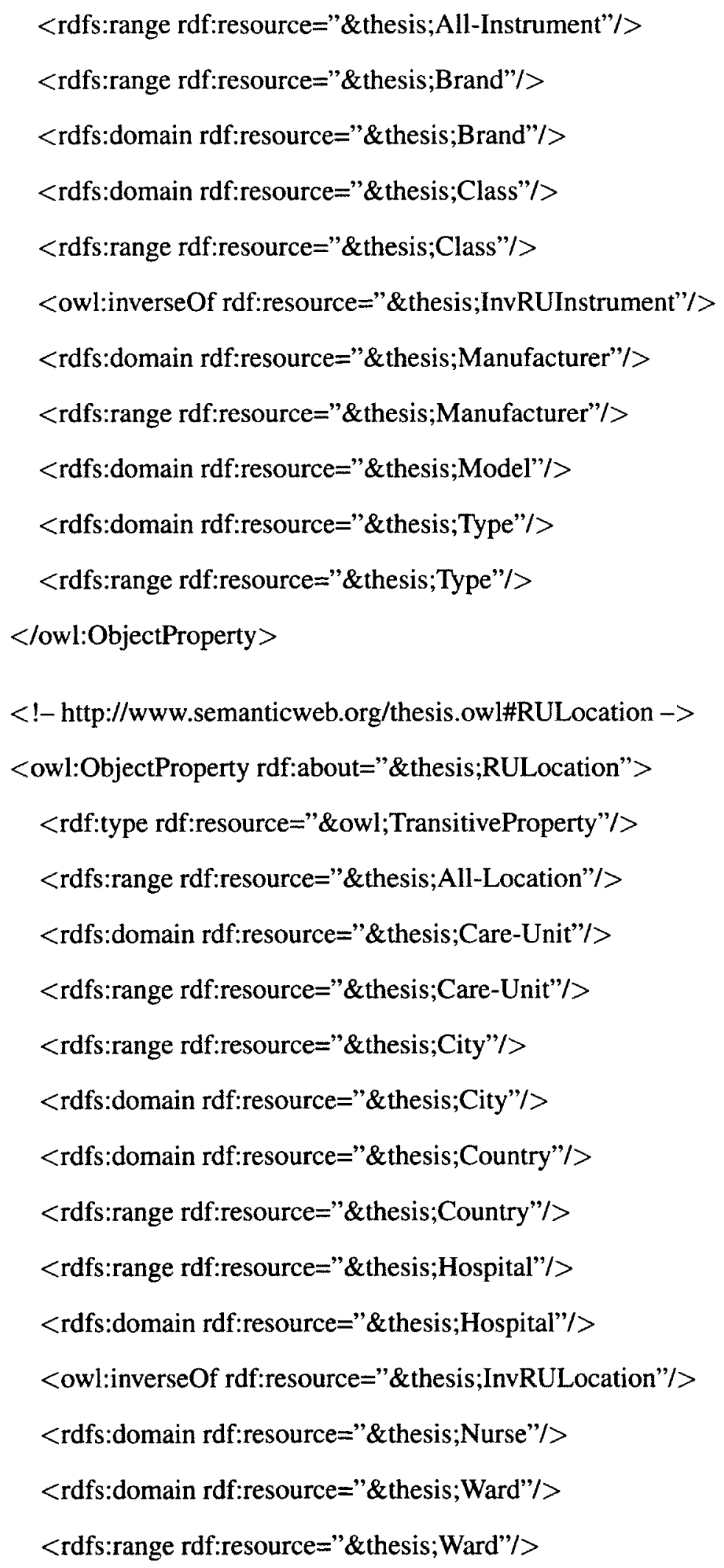




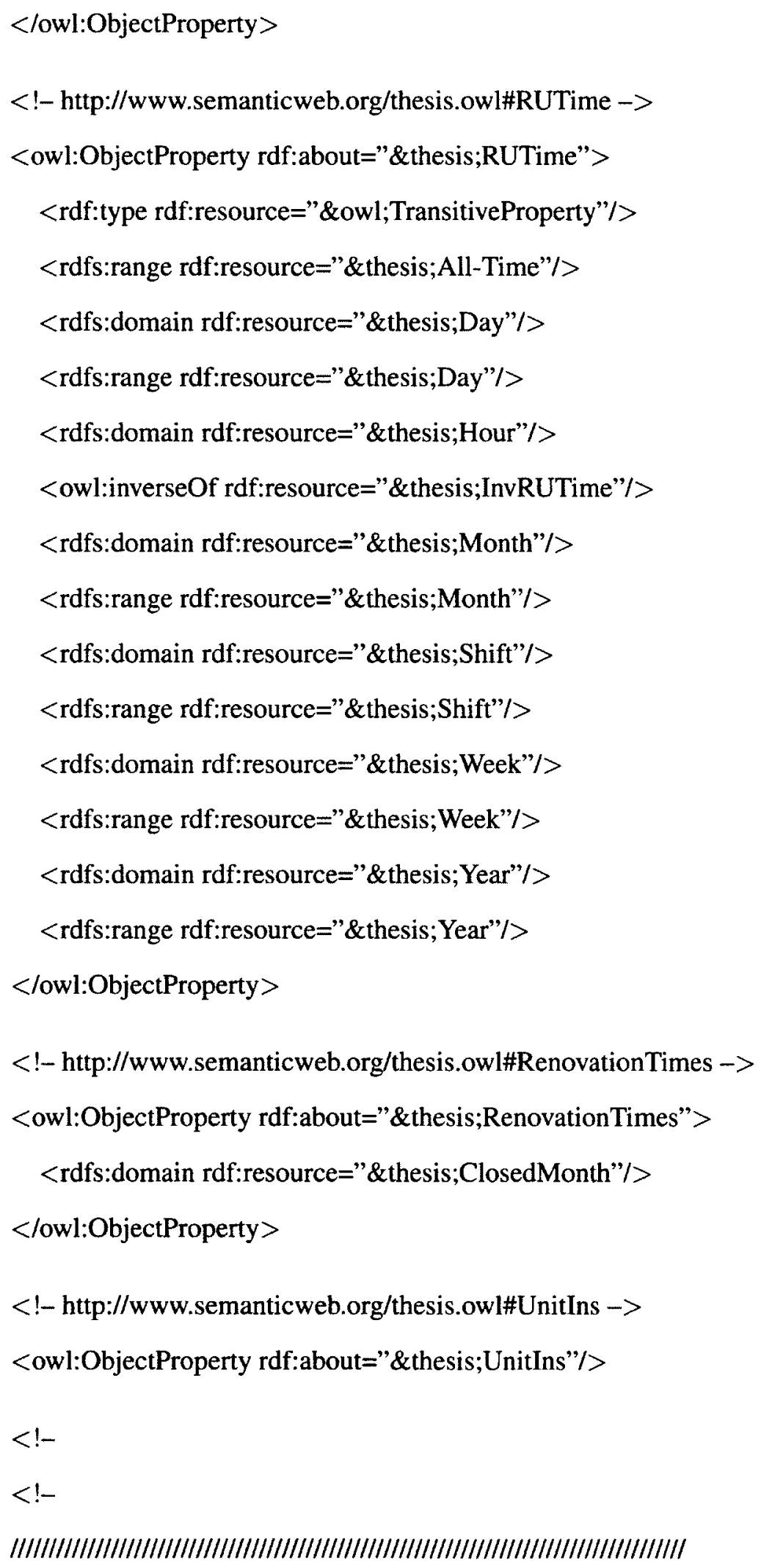


// Data properties

/I

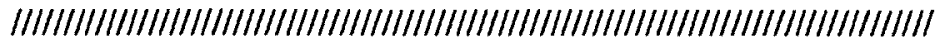

$\rightarrow$

$<$ !- http://www.semanticweb.org/thesis.owl\#HospitalSystem $->$

<owl:DatatypeProperty rdf:about="http://www.semanticweb.org/thesis.owl\#HospitalSystem"> <rdfs:domain rdf:resource="\&thesis;Hospital"/>

<rdfs:range rdf:resource="\&xsd;string"/>

$<$ lowl:DatatypeProperty $>$

$<!-$ http://www.semanticweb.org/thesis.owl\#HospitalType $\rightarrow>$

<owl:DatatypeProperty rdf:about="http://www.semanticweb.org/thesis.owl\#HospitalType"> < rdfs:domain rdf:resource="\&thesis;Hospital"/>

<rdfs:range rdf:resource="\&xsd;string"/>

$<$ lowl:DatatypeProperty $>$

$<!-$

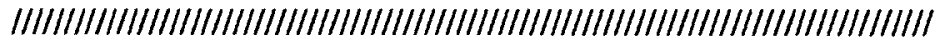

/I

// Classes

/I

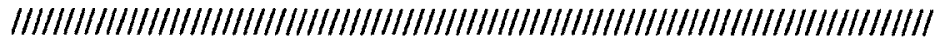

$\rightarrow$

$<!-$ http://www.semanticweb.org/thesis.owl\#All-Instrument ->

<owl:Class rdf:about="http://www.semanticweb.org/thesis.owl\#All-Instrument">

$<$ rdfs:subClassOf $>$

<owl:Restriction>

<owl:onProperty rdf:resource="http://www.semanticweb.org/thesis.owl\#InvRUInstrument"/> 


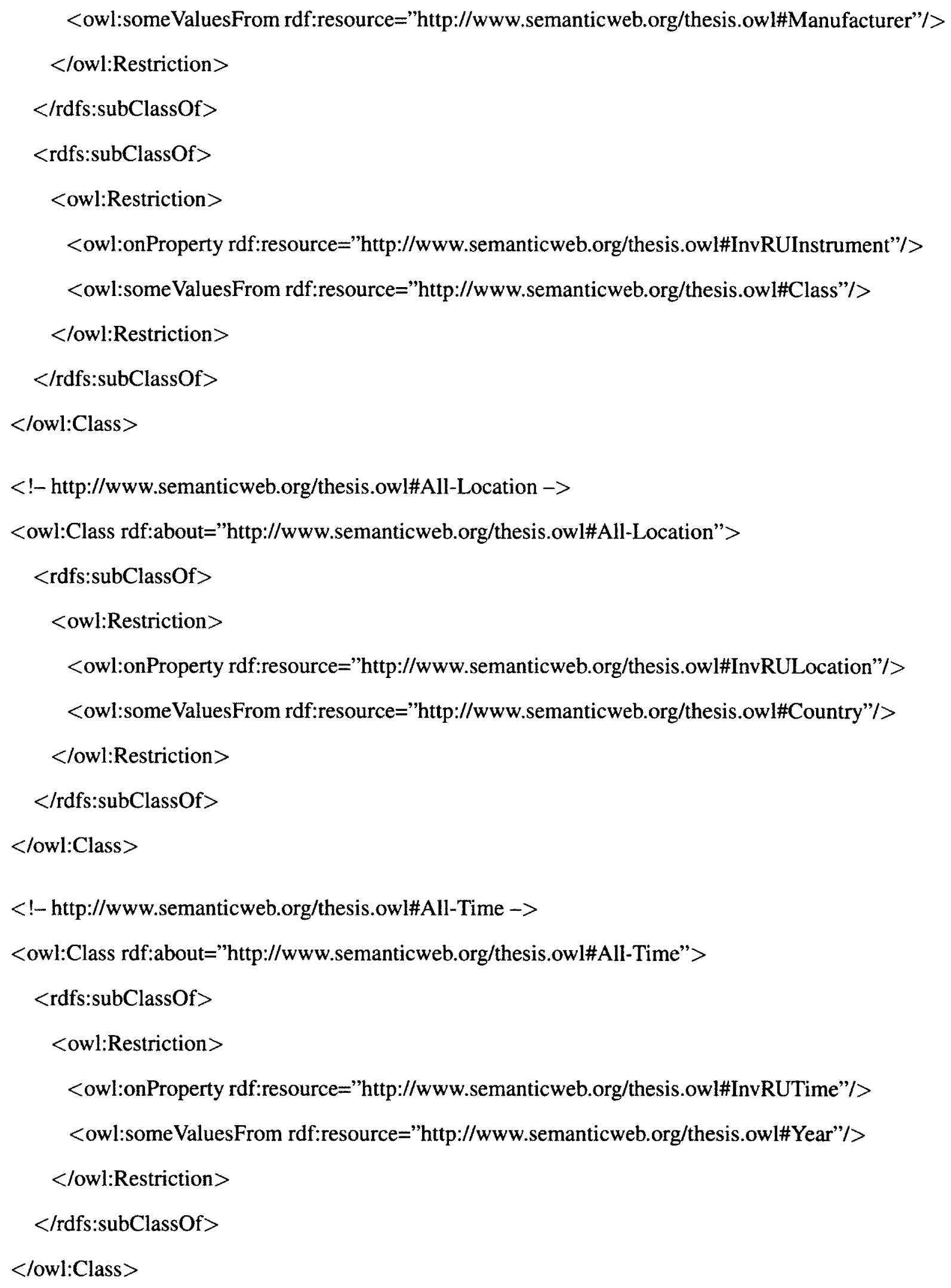




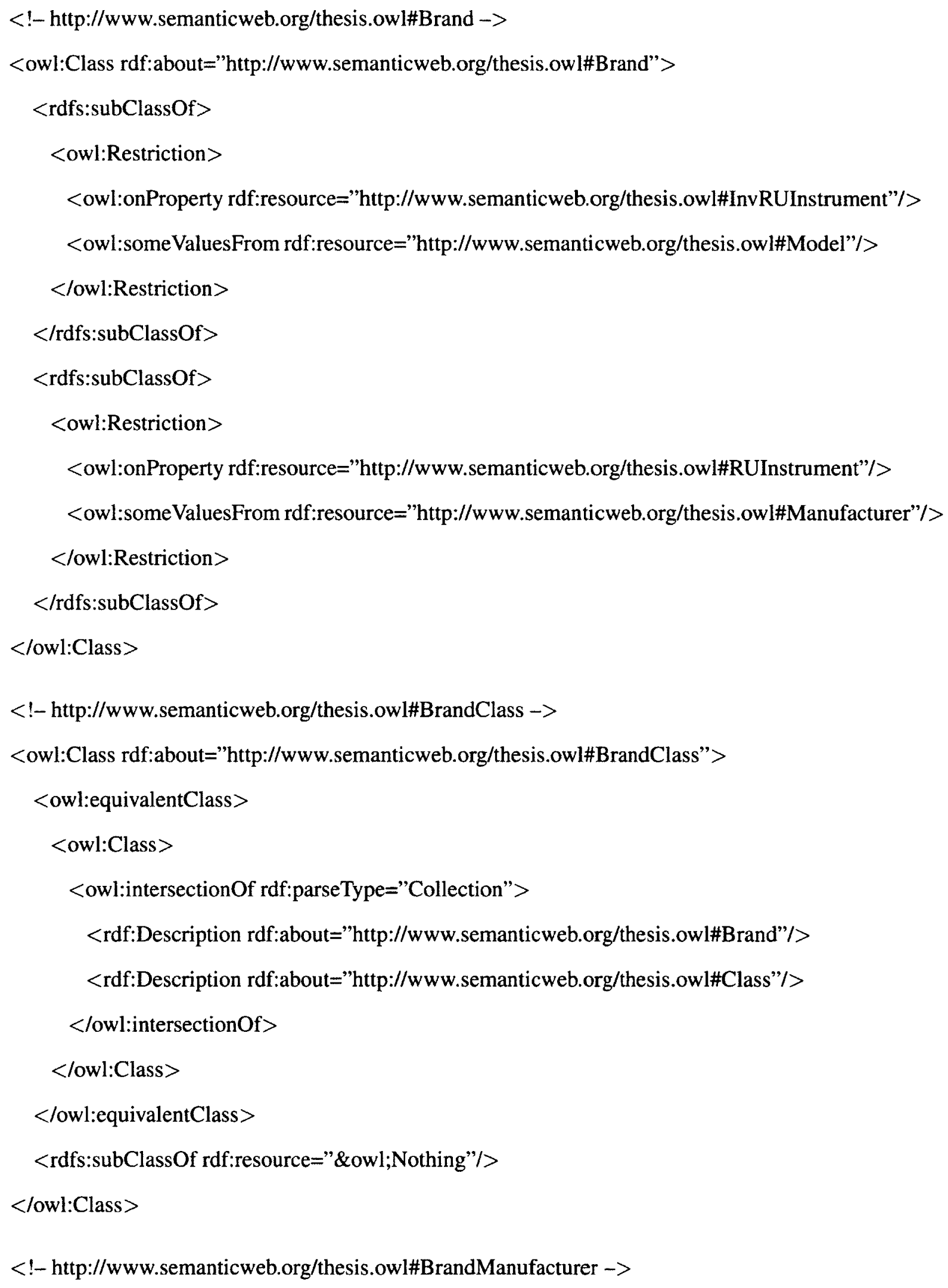




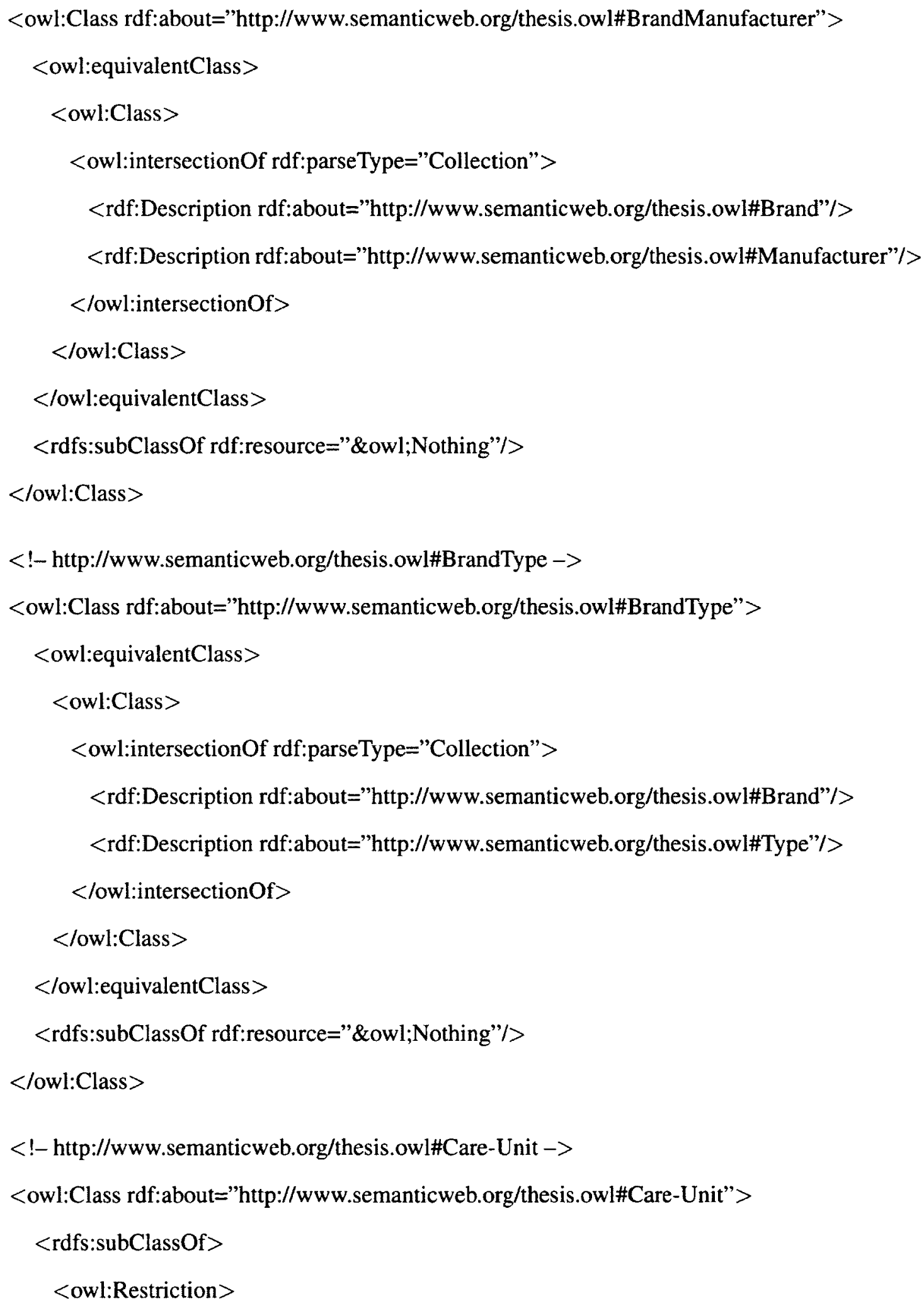




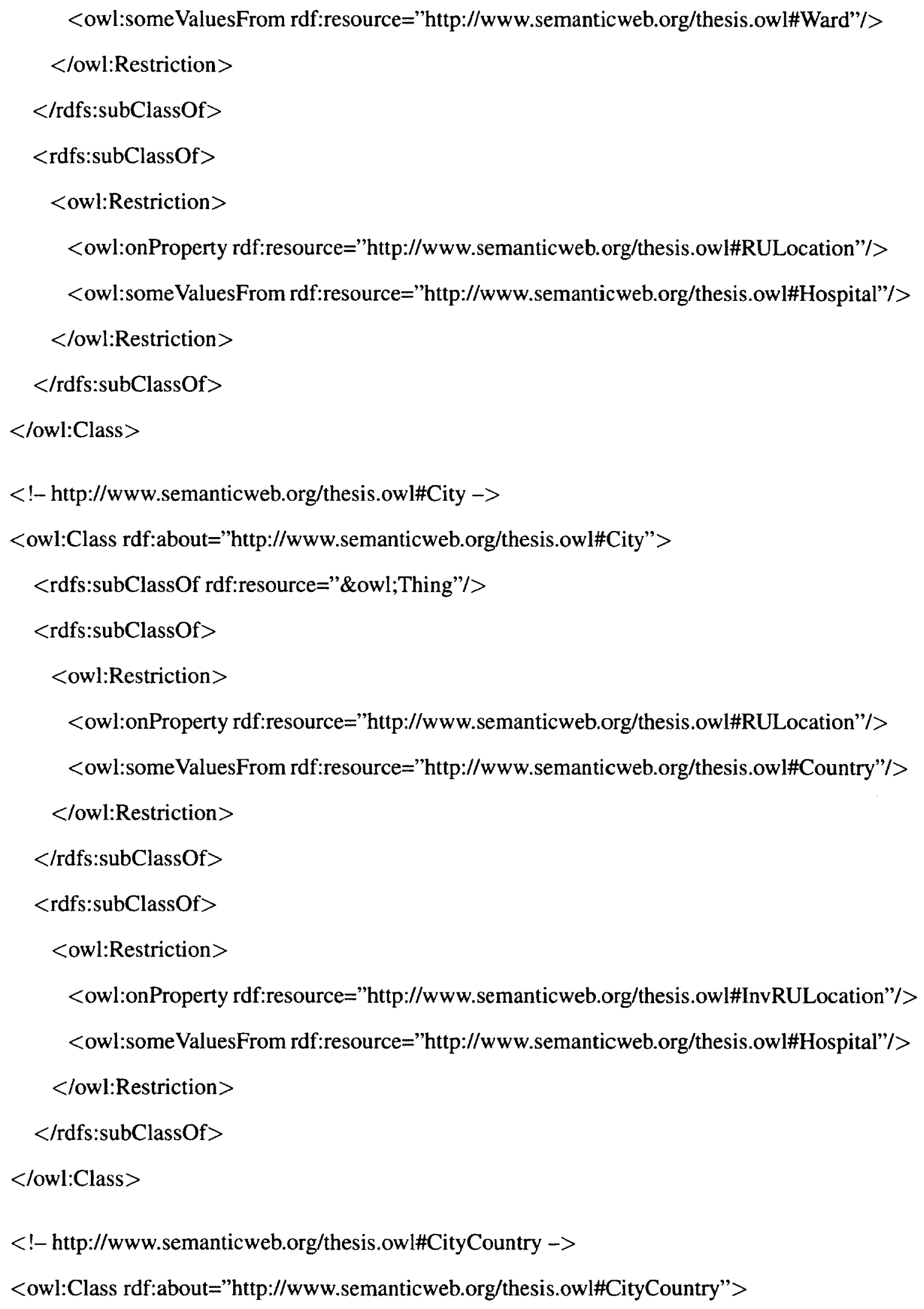




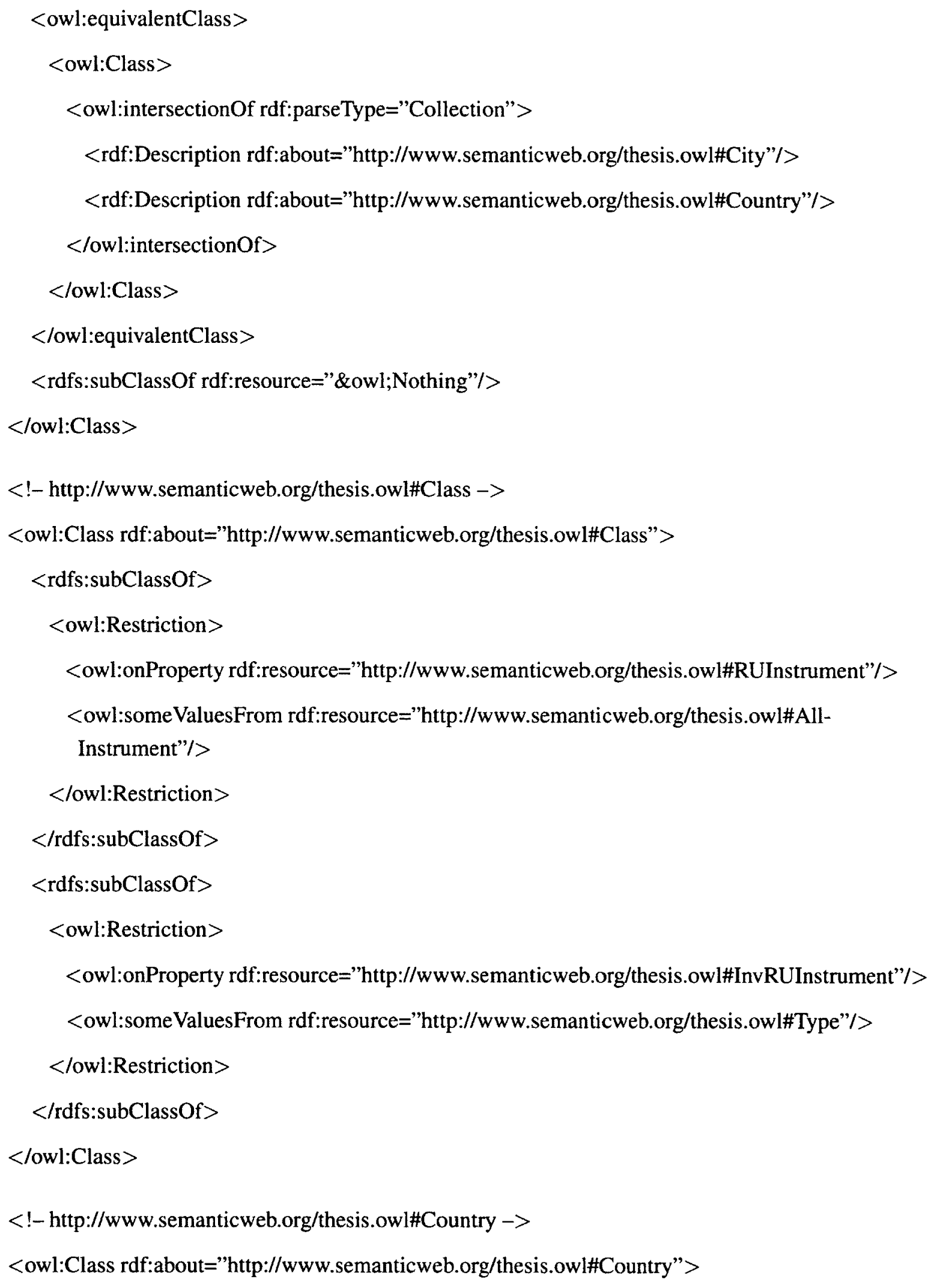




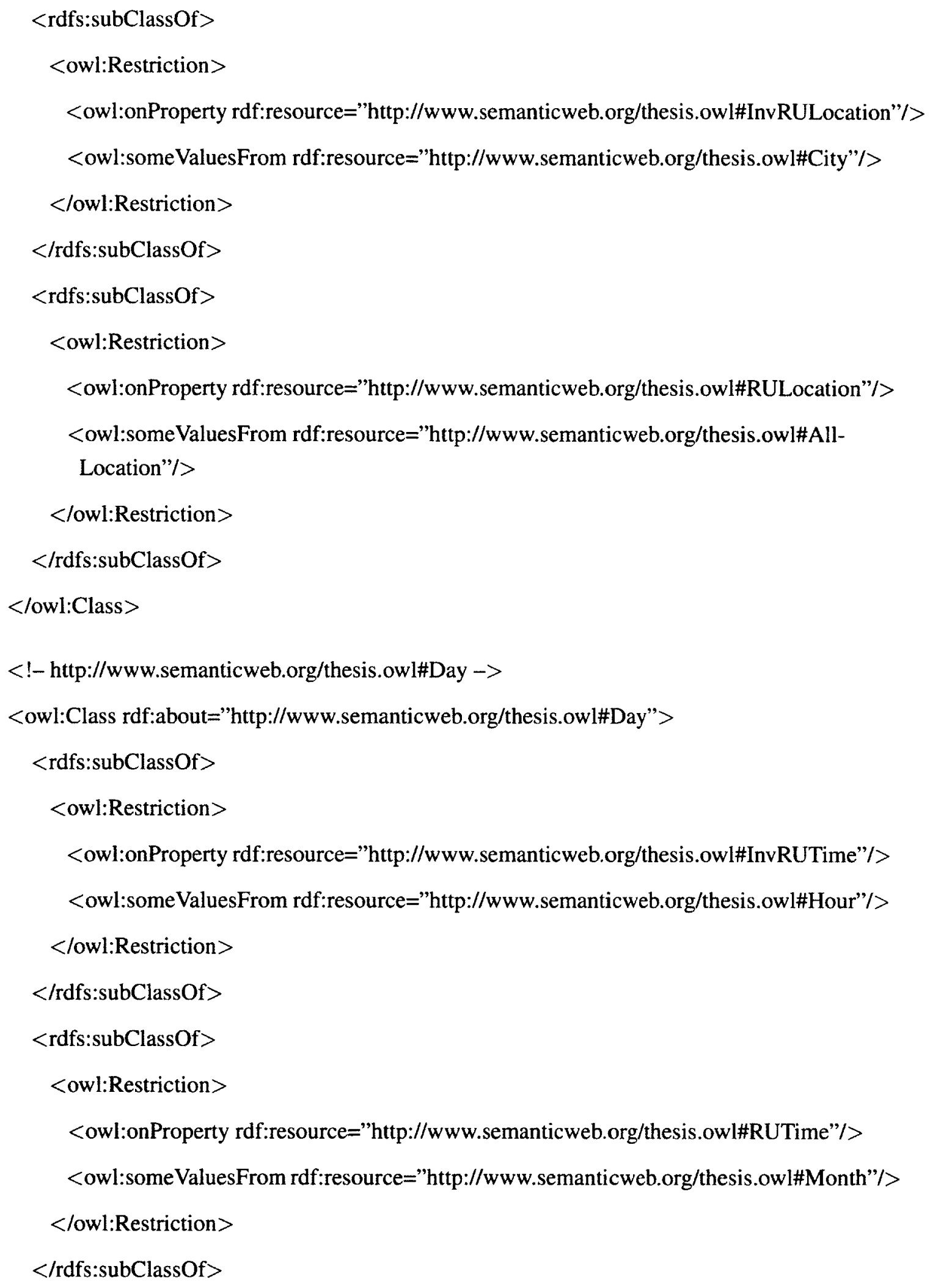




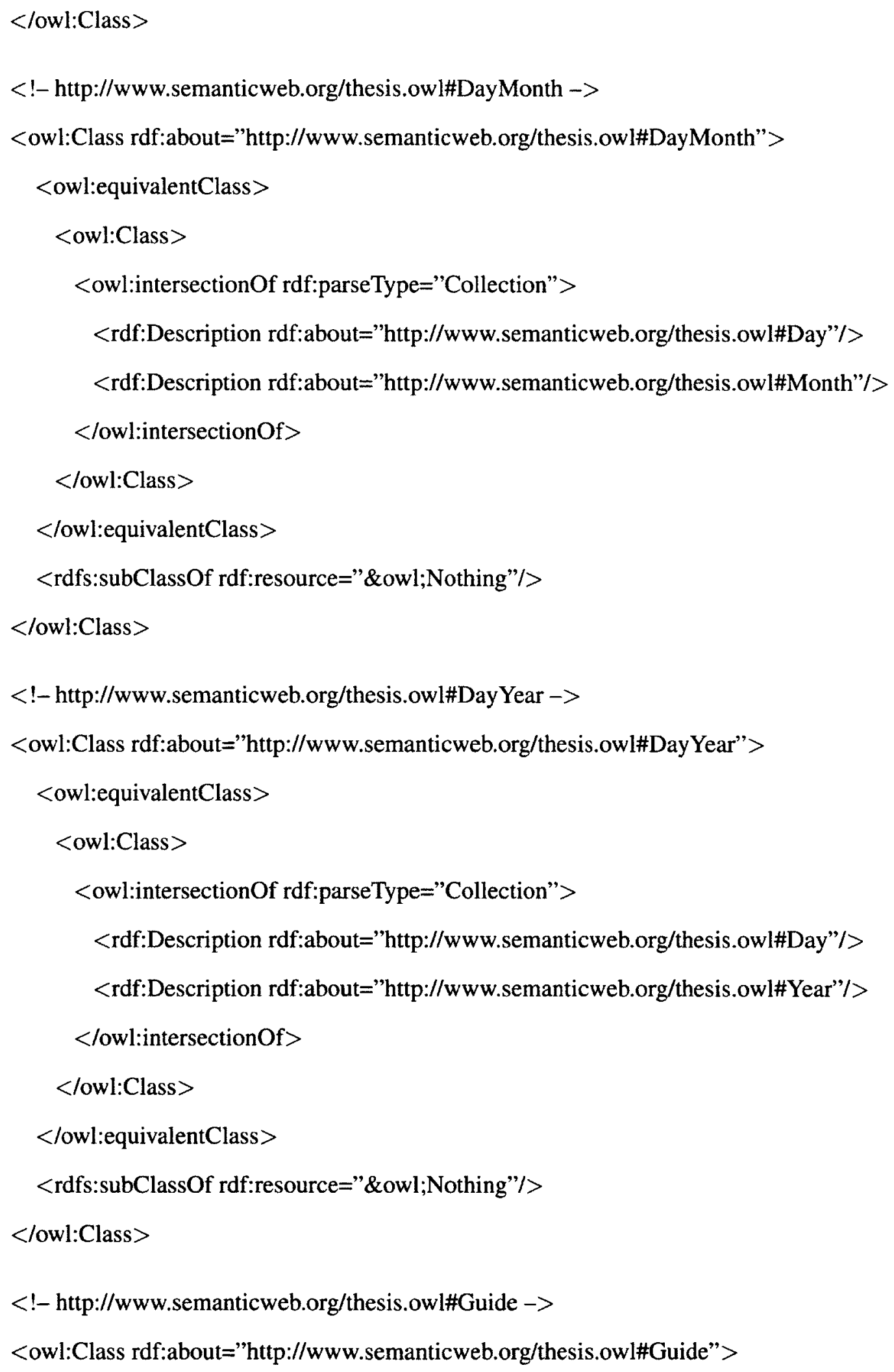




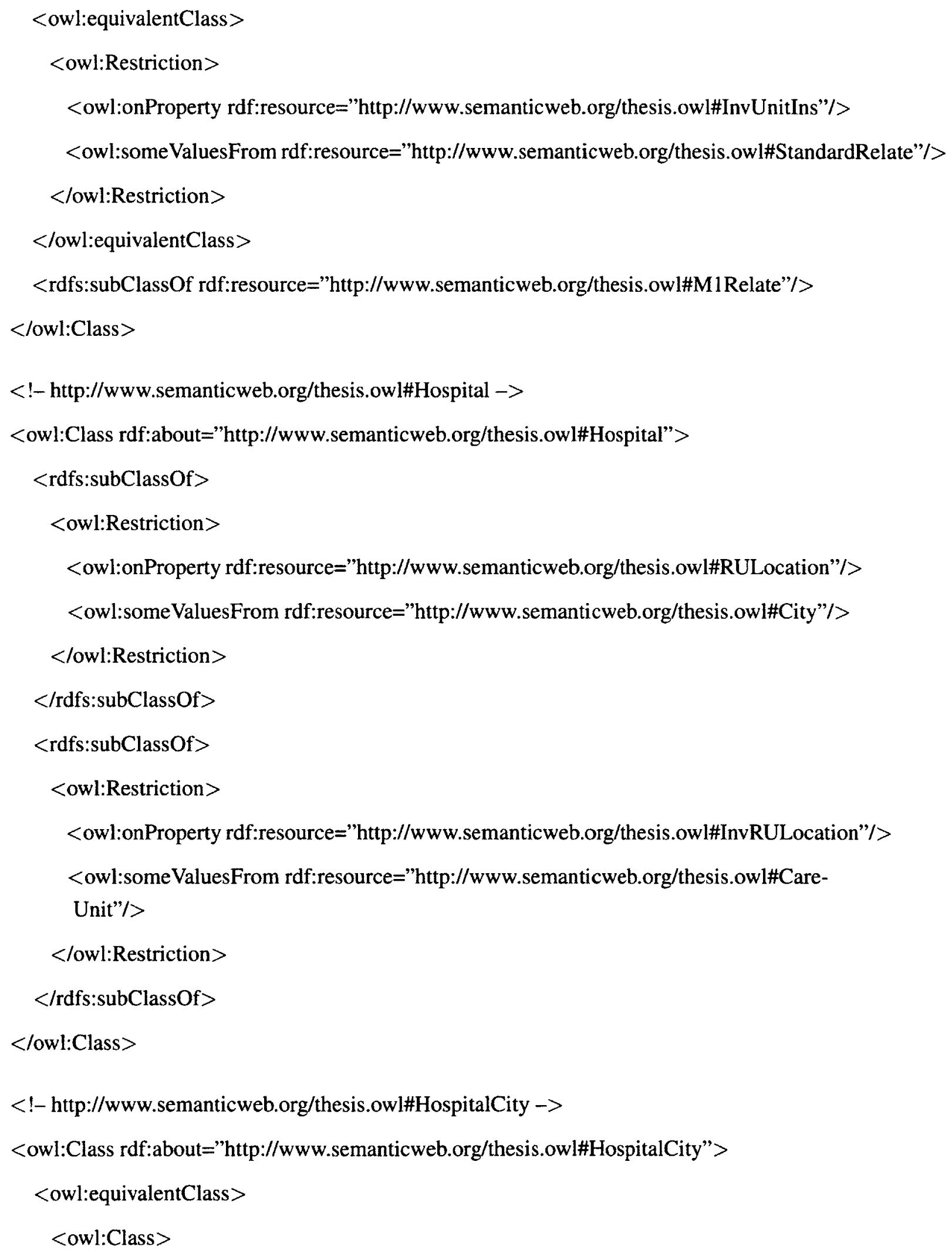




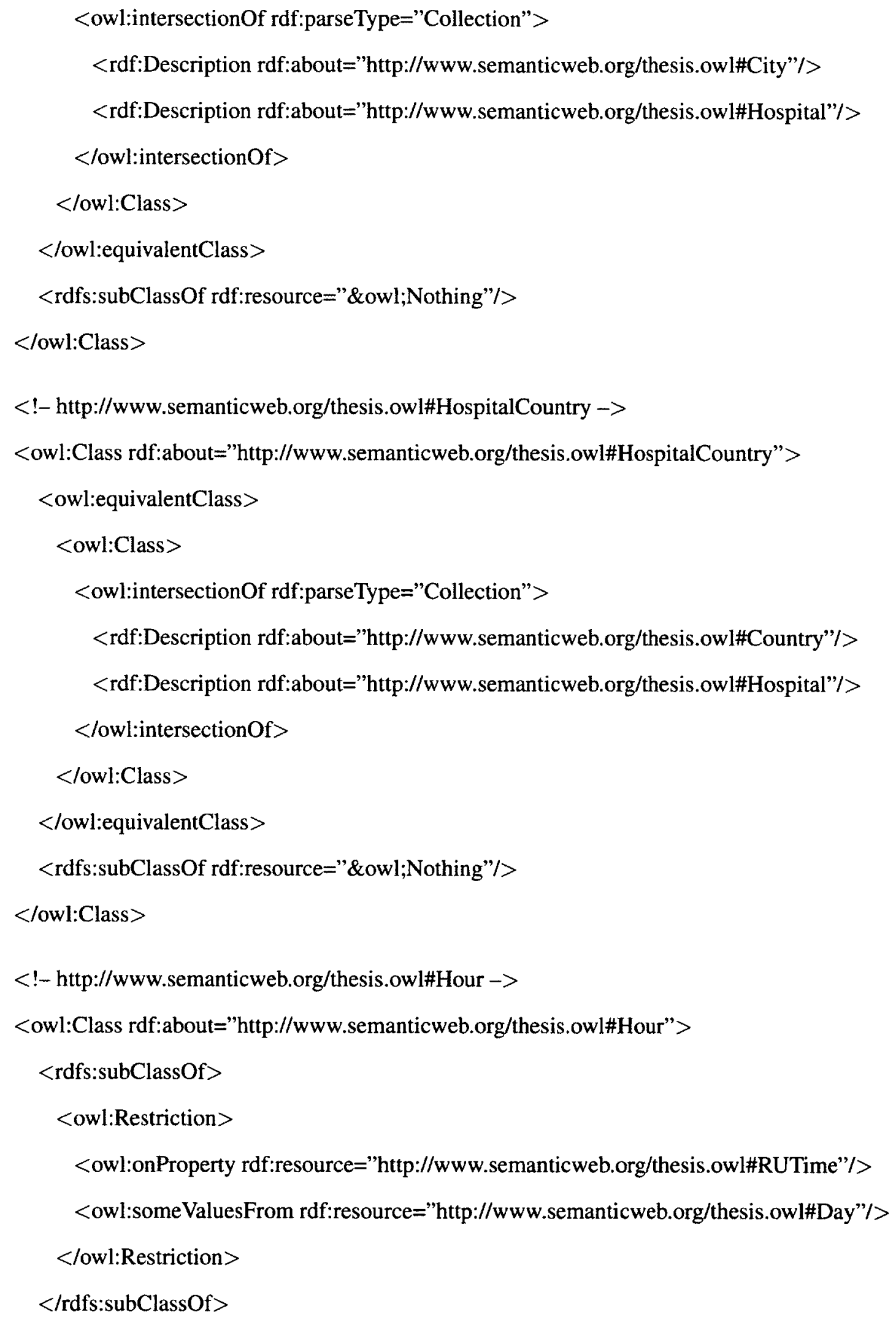




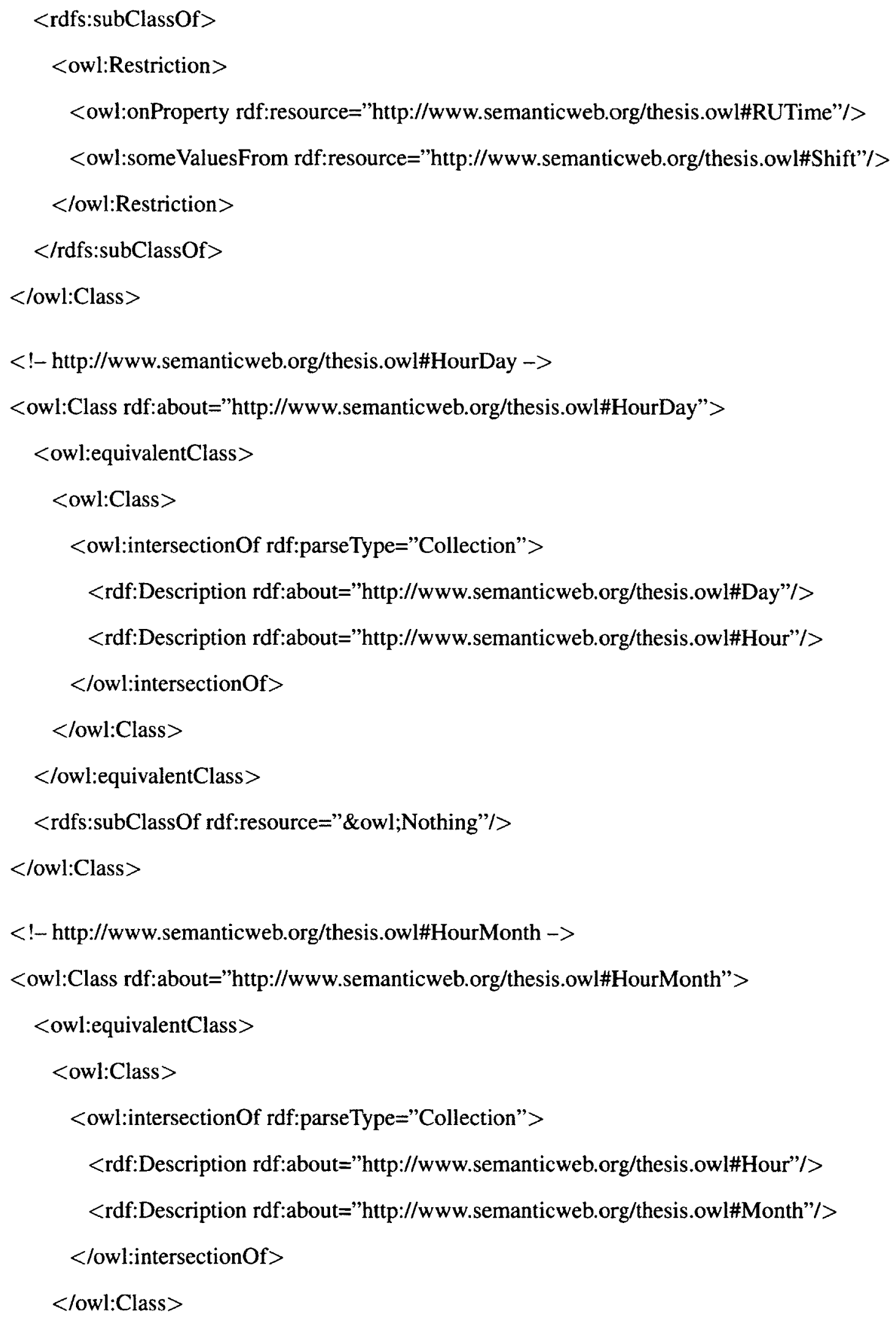




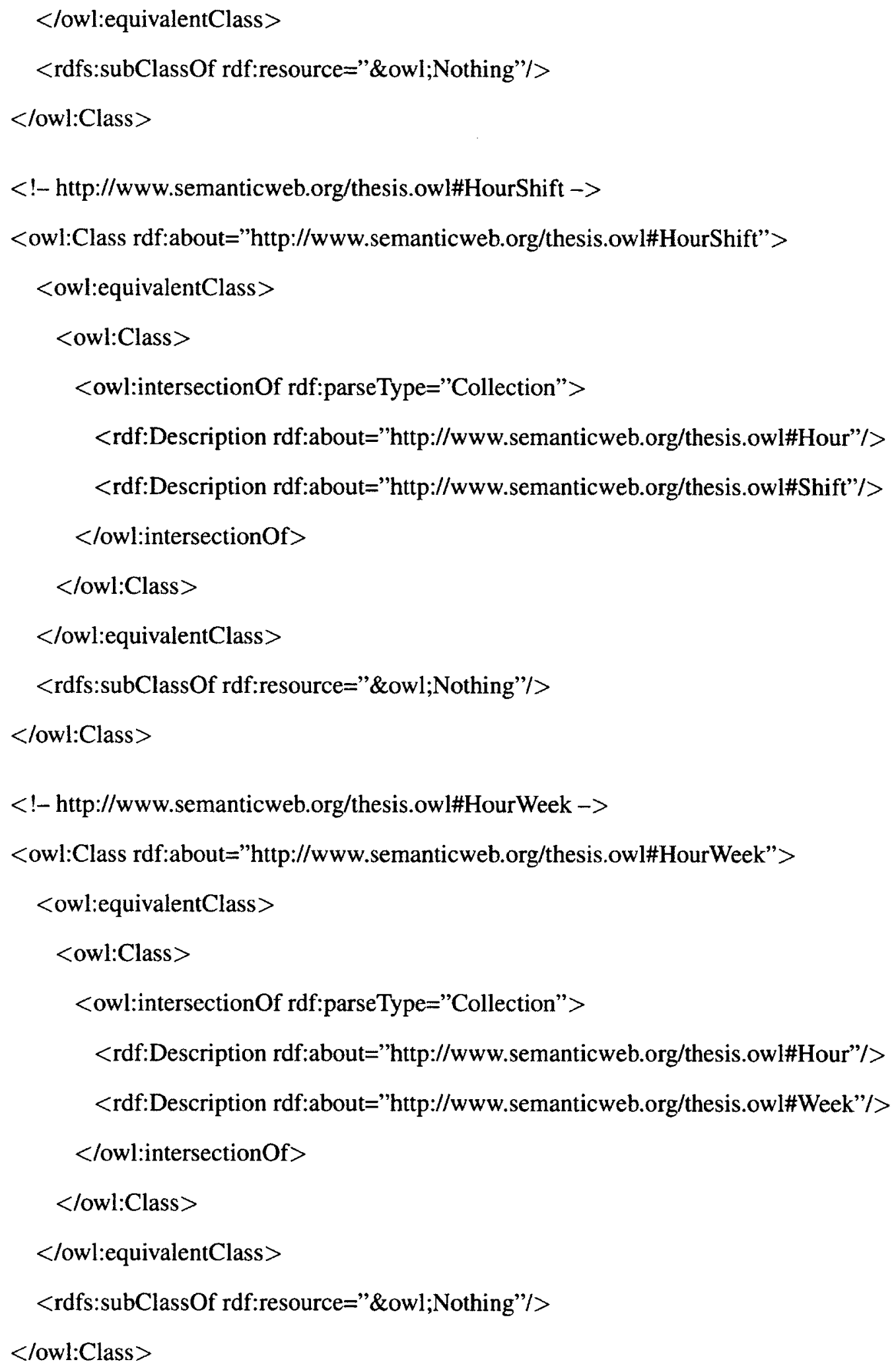




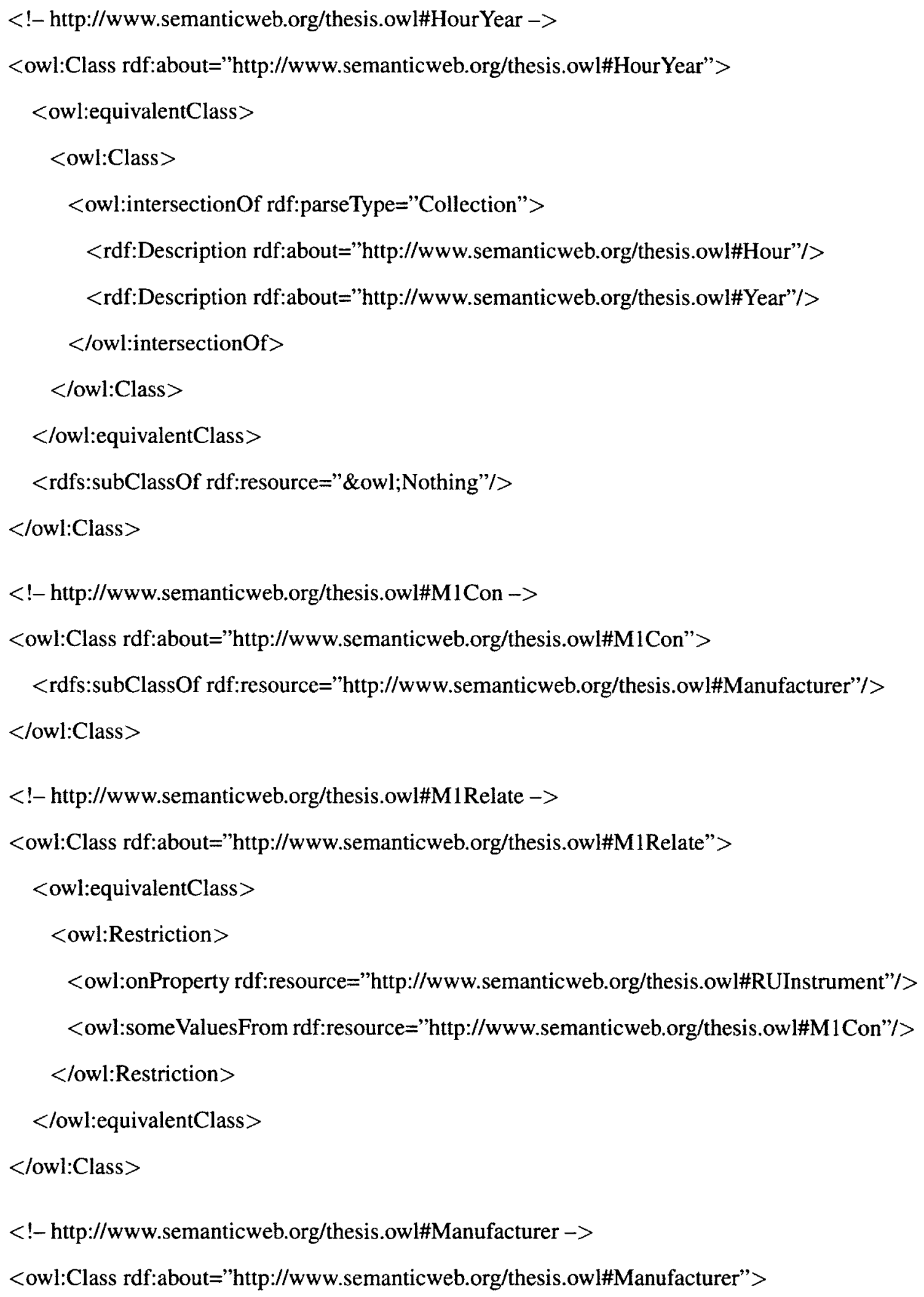




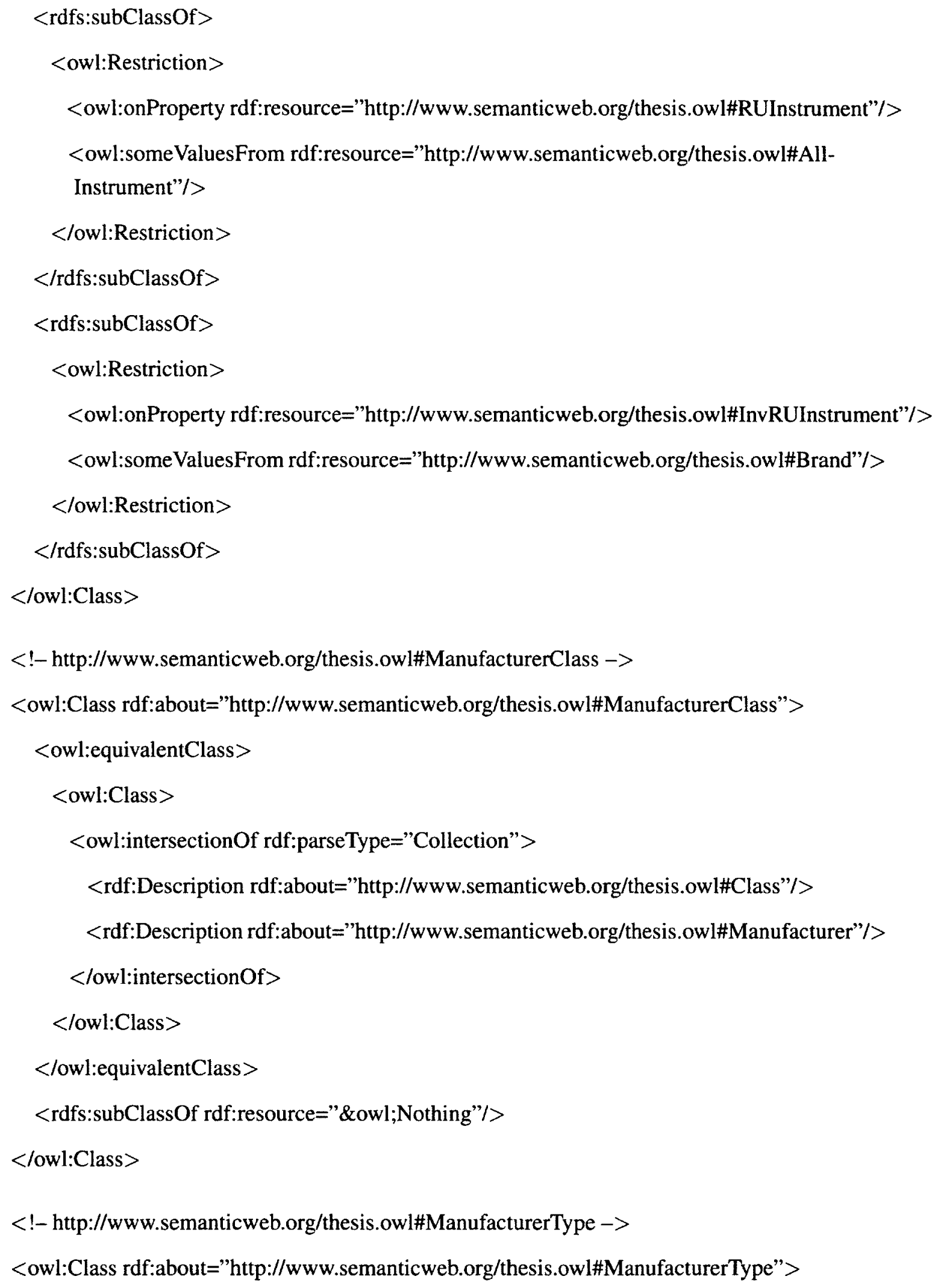




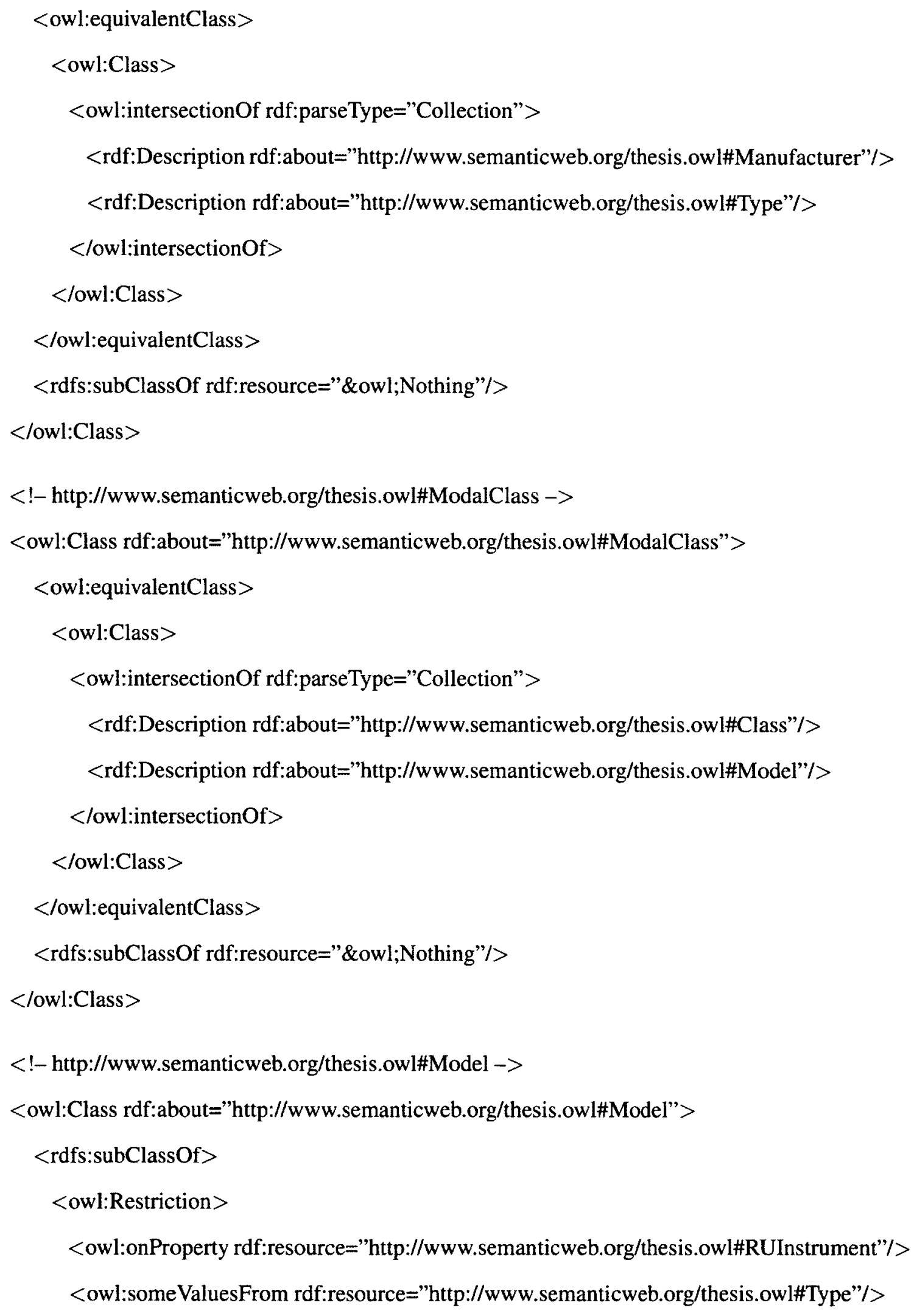




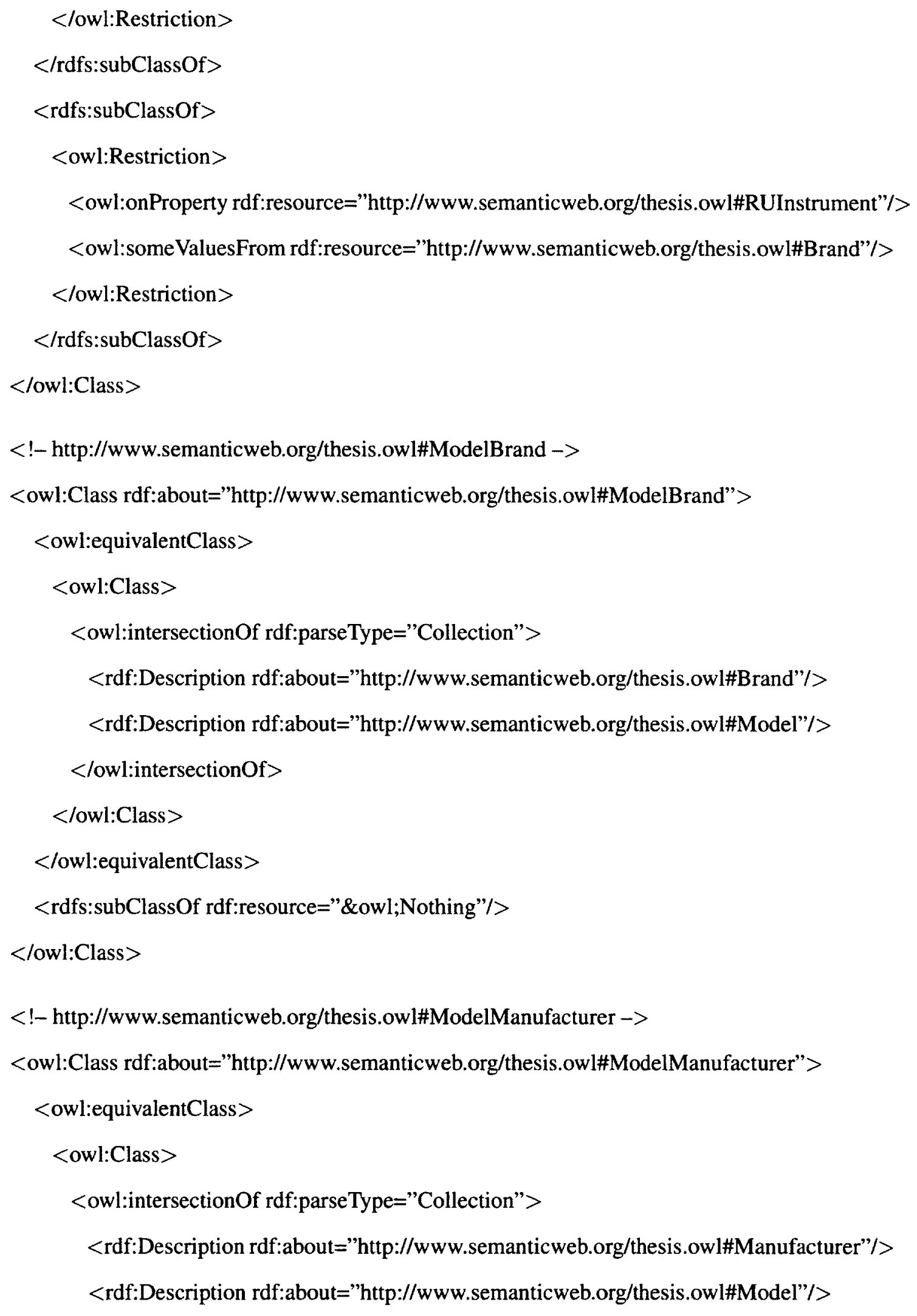




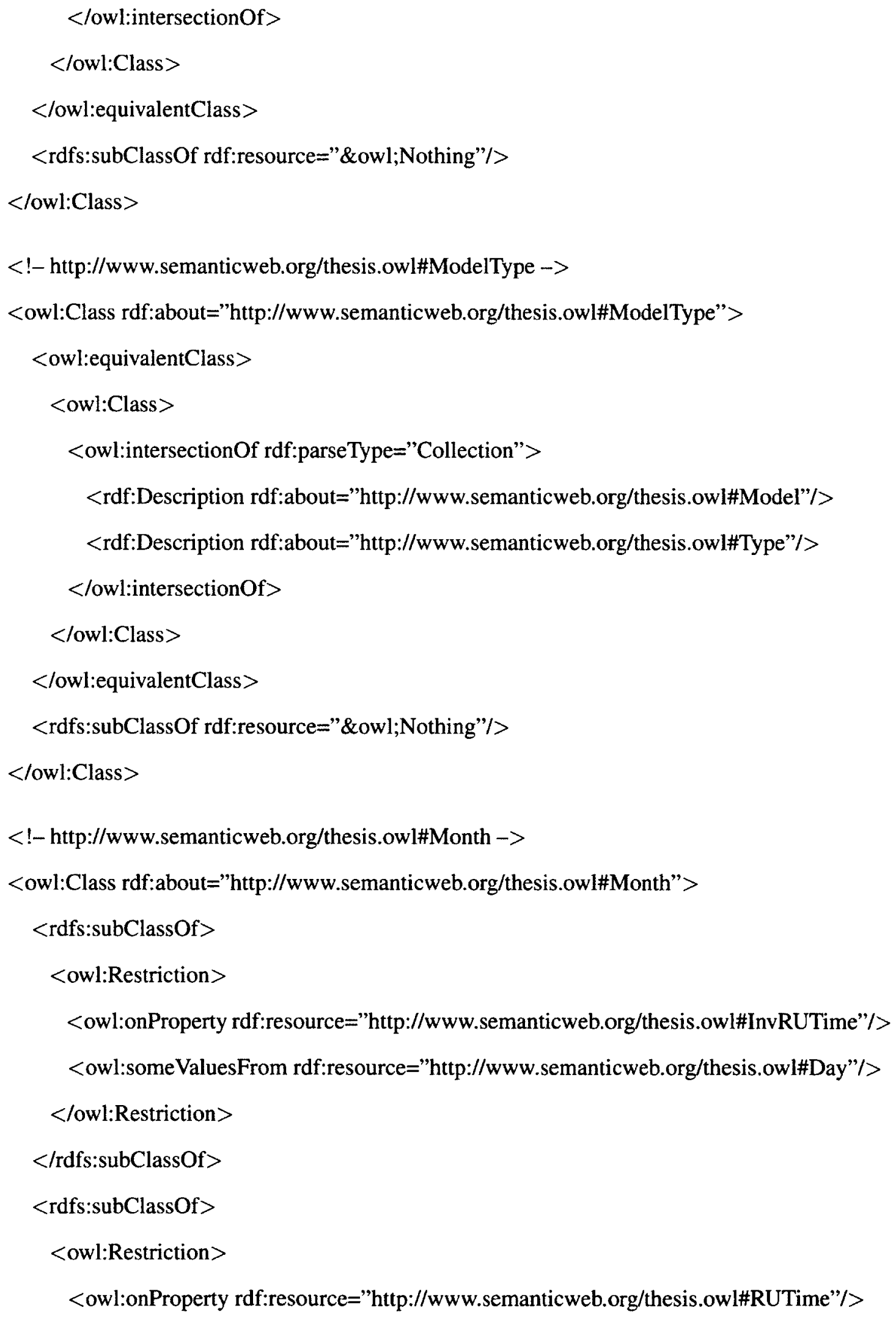




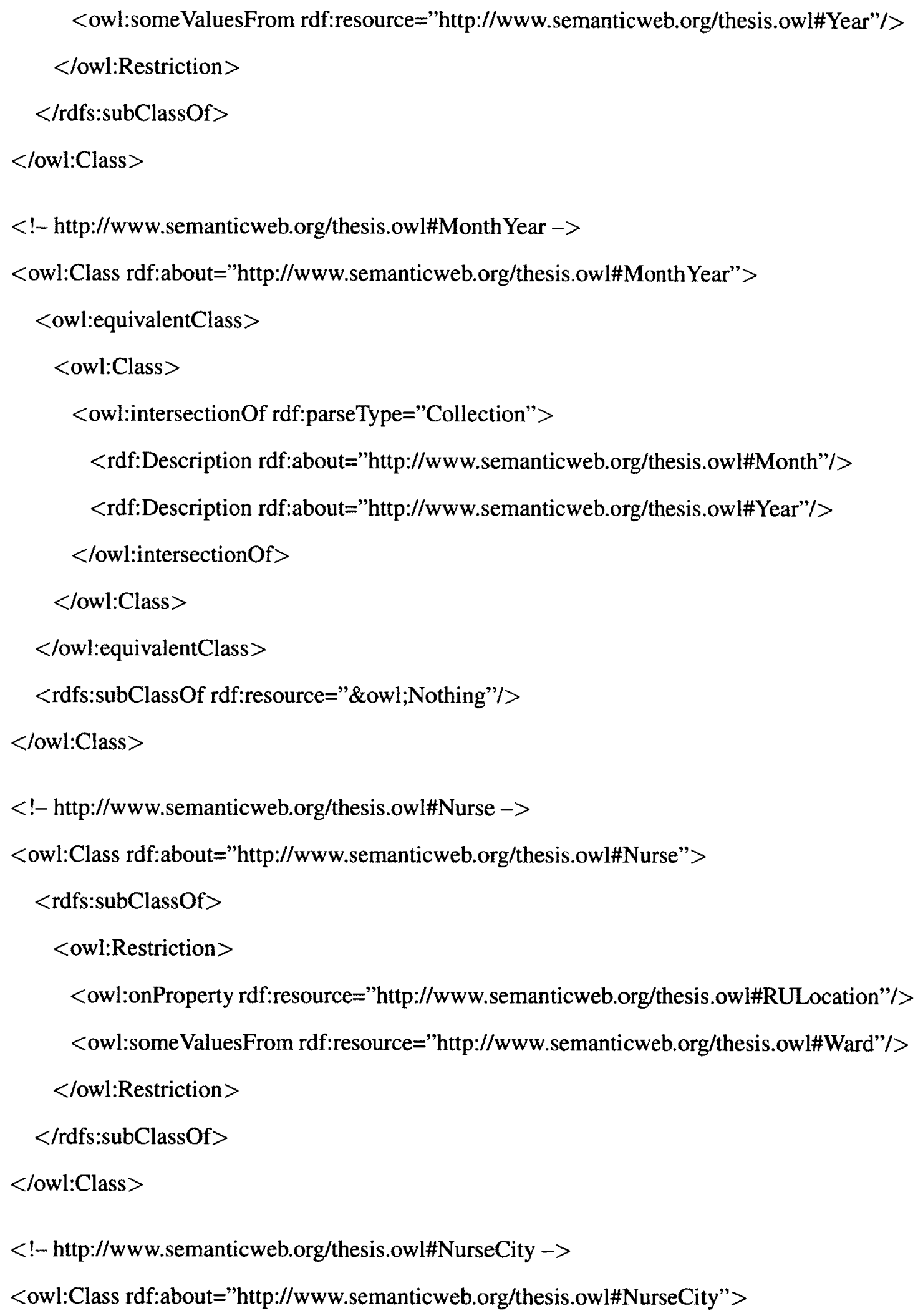




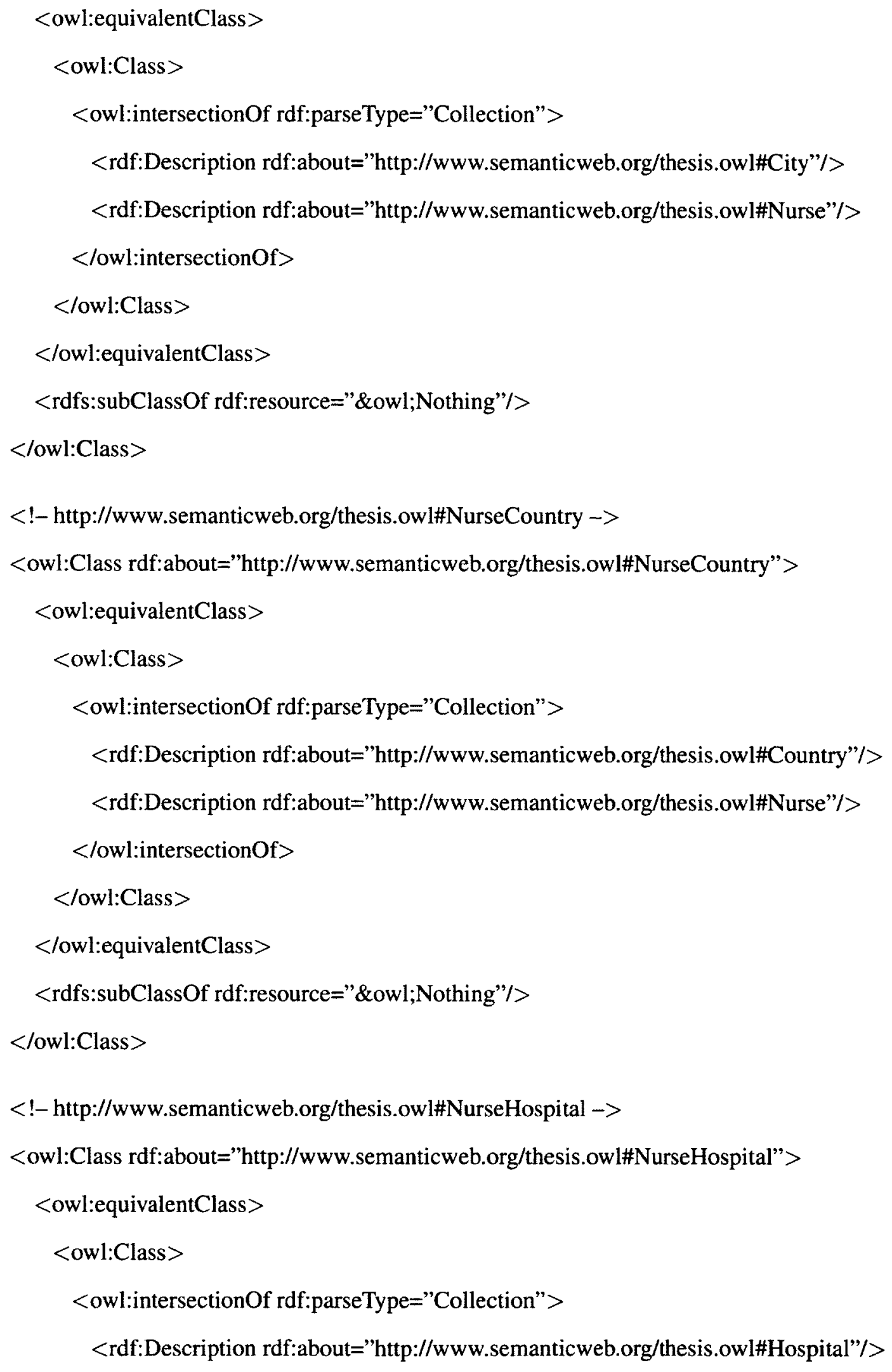




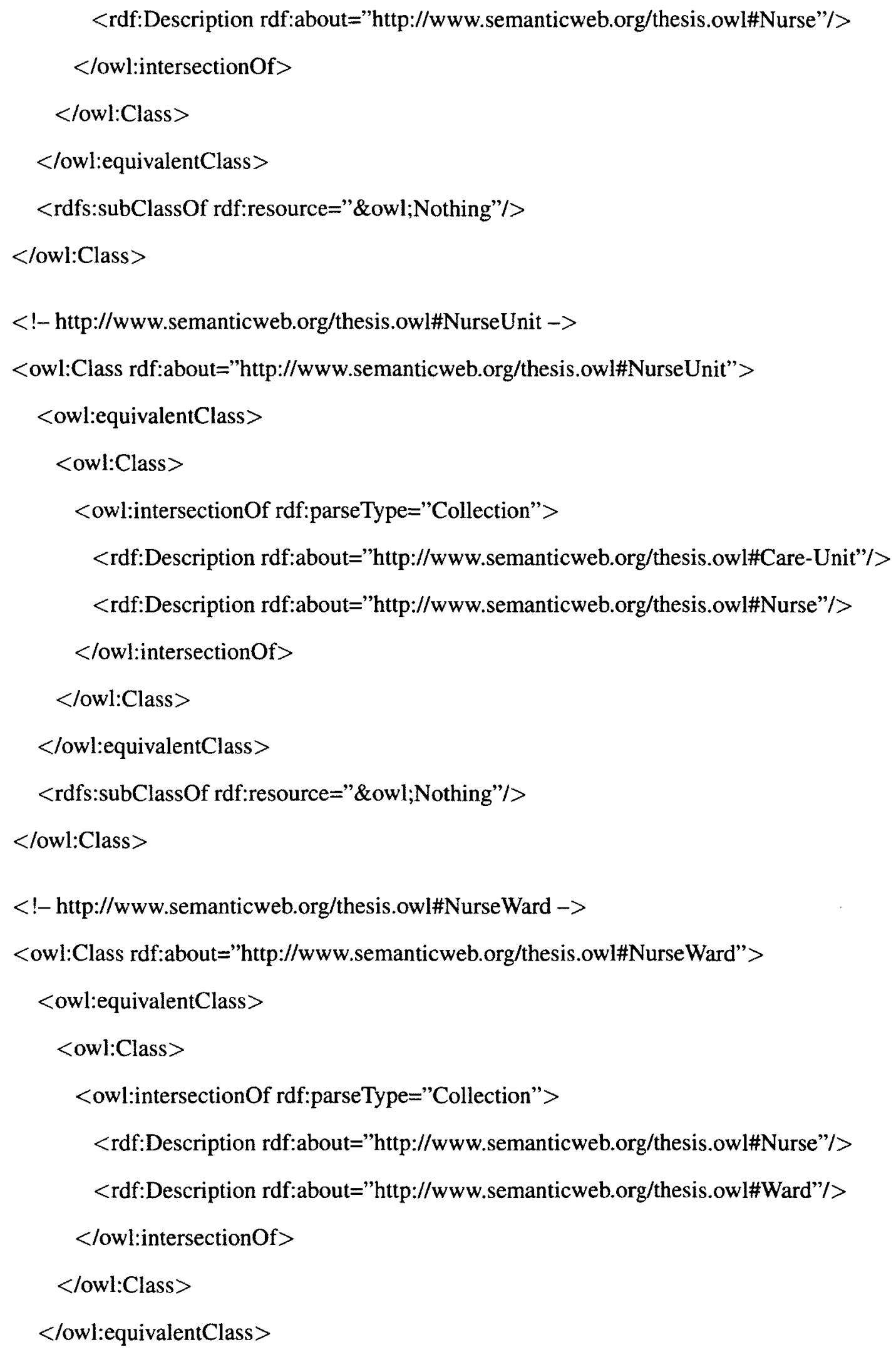




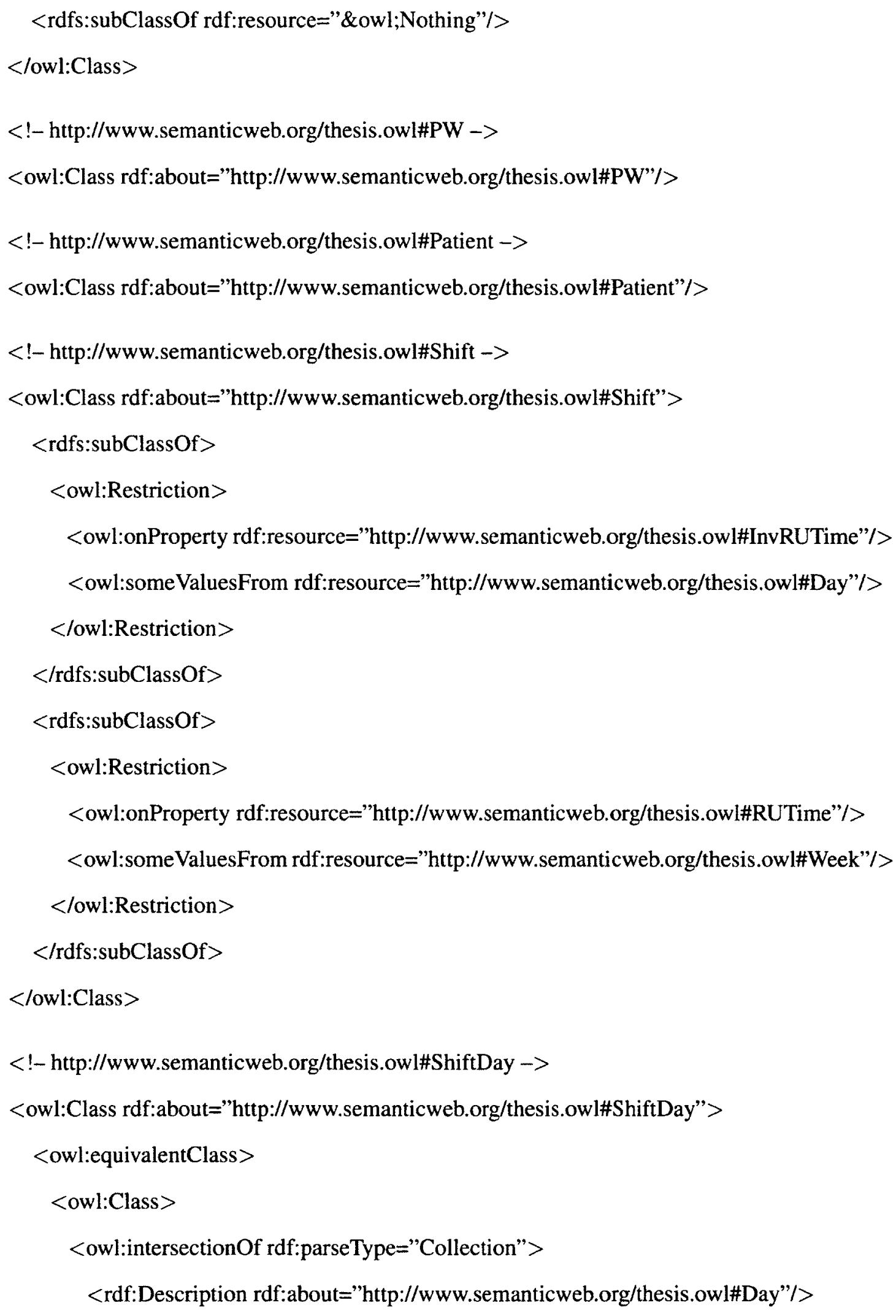




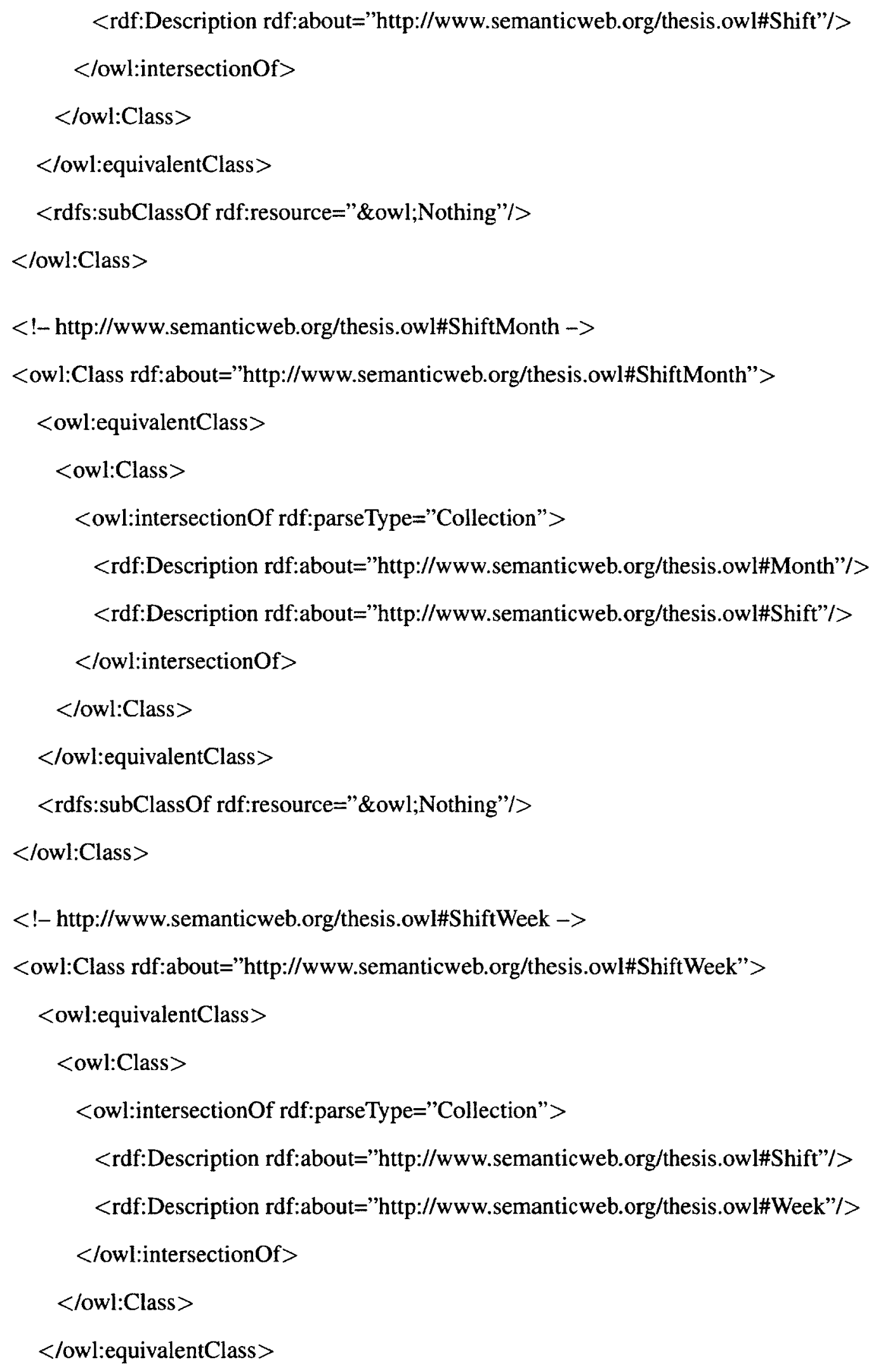




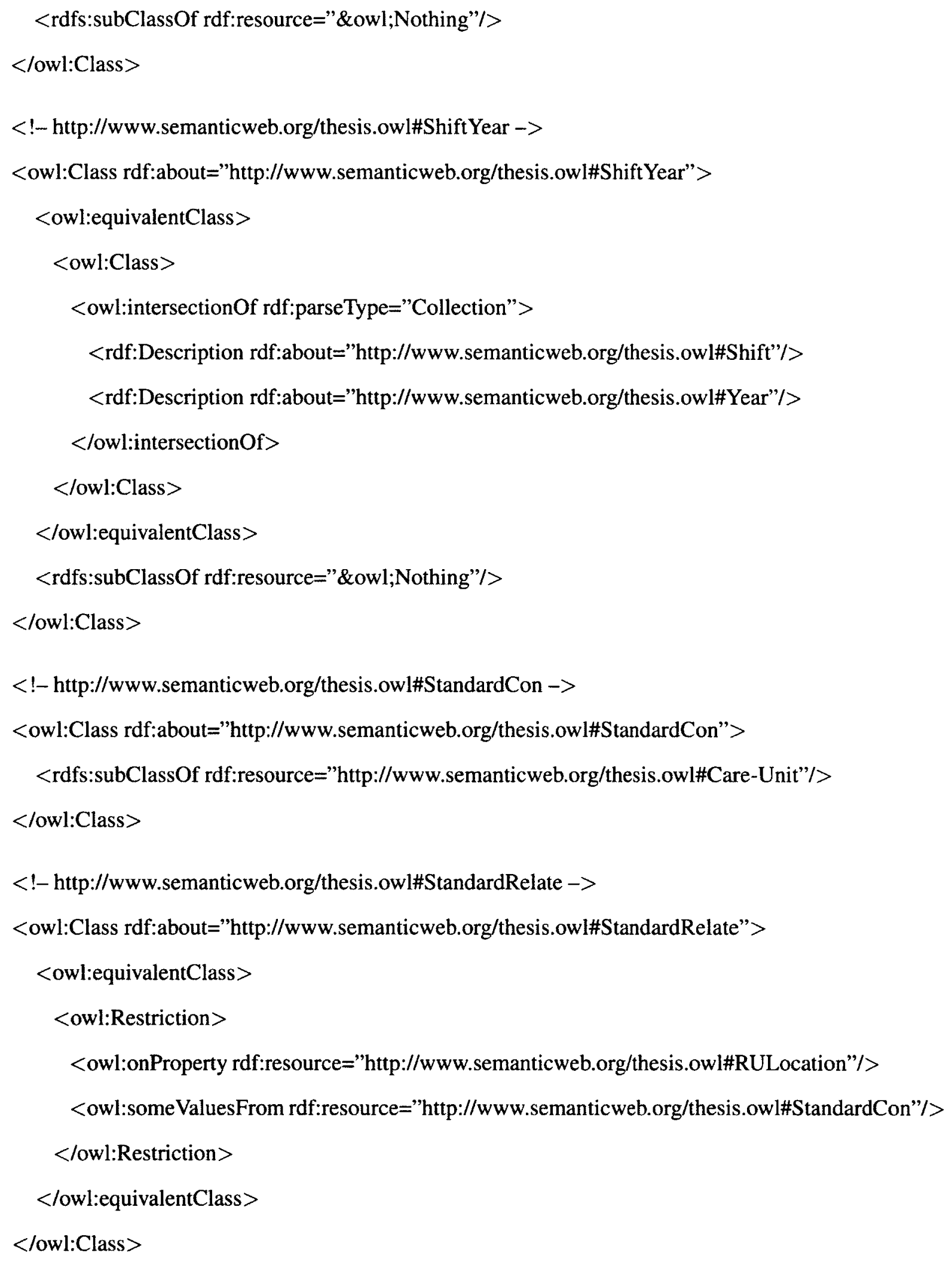




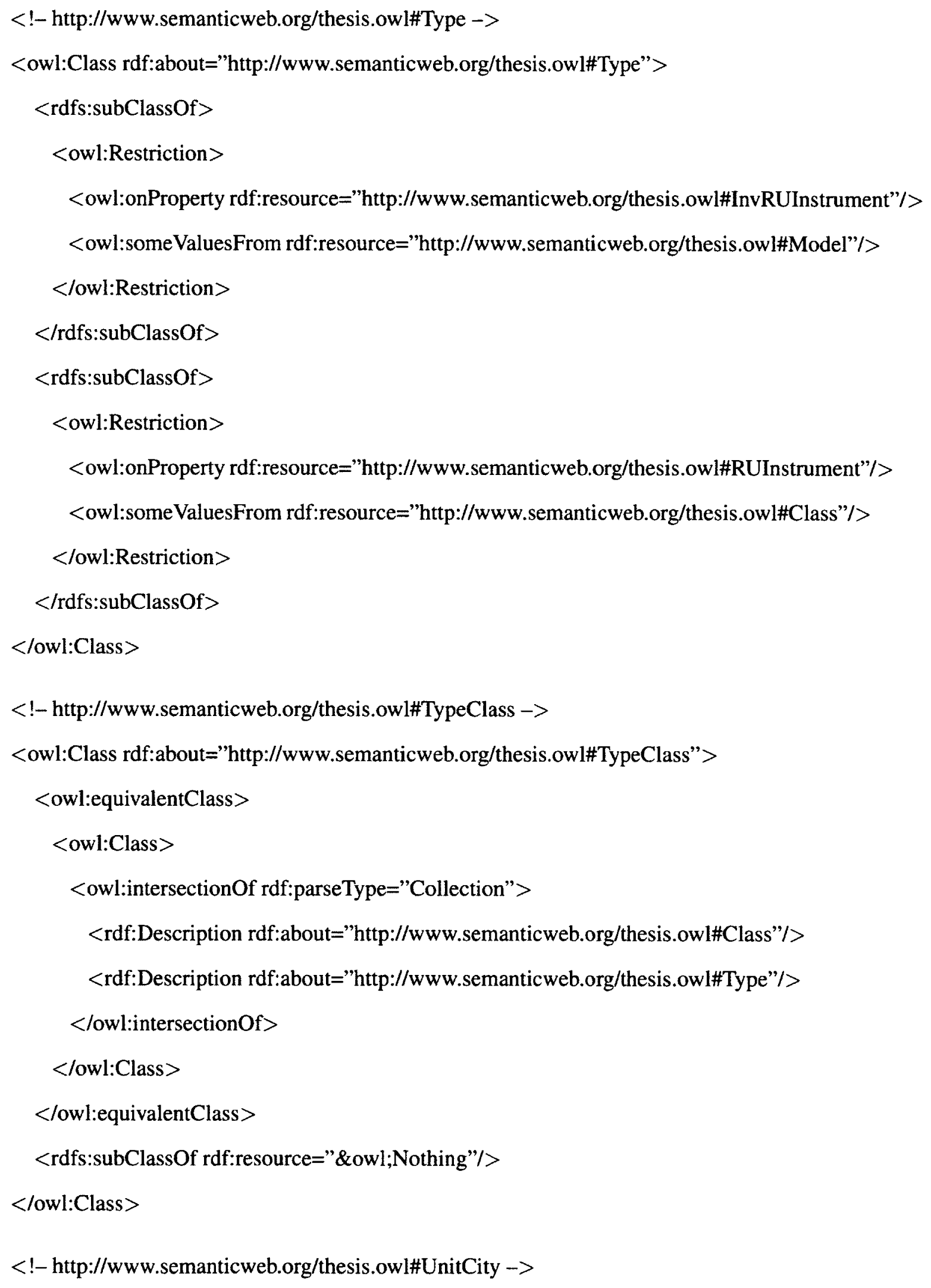




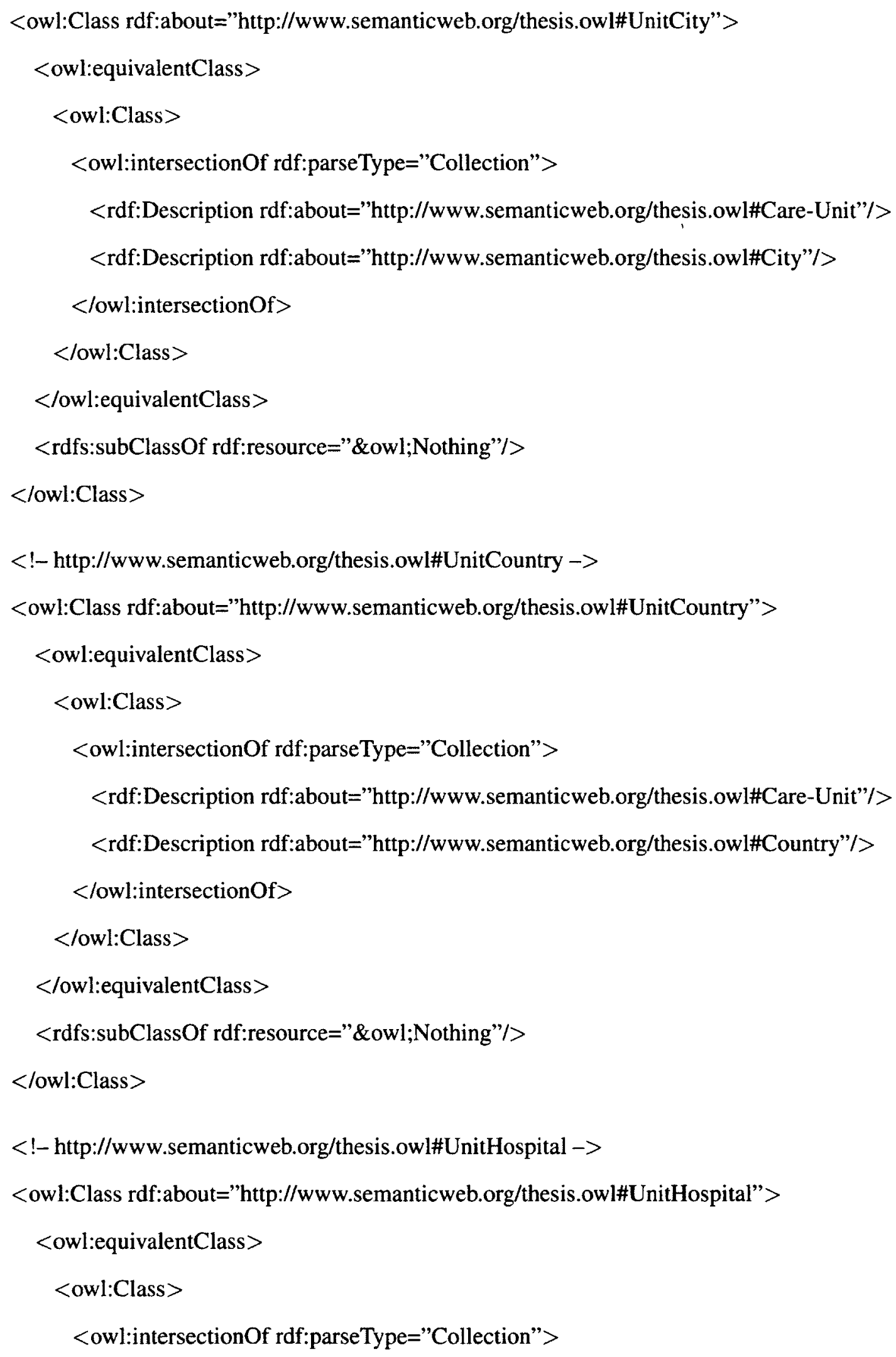




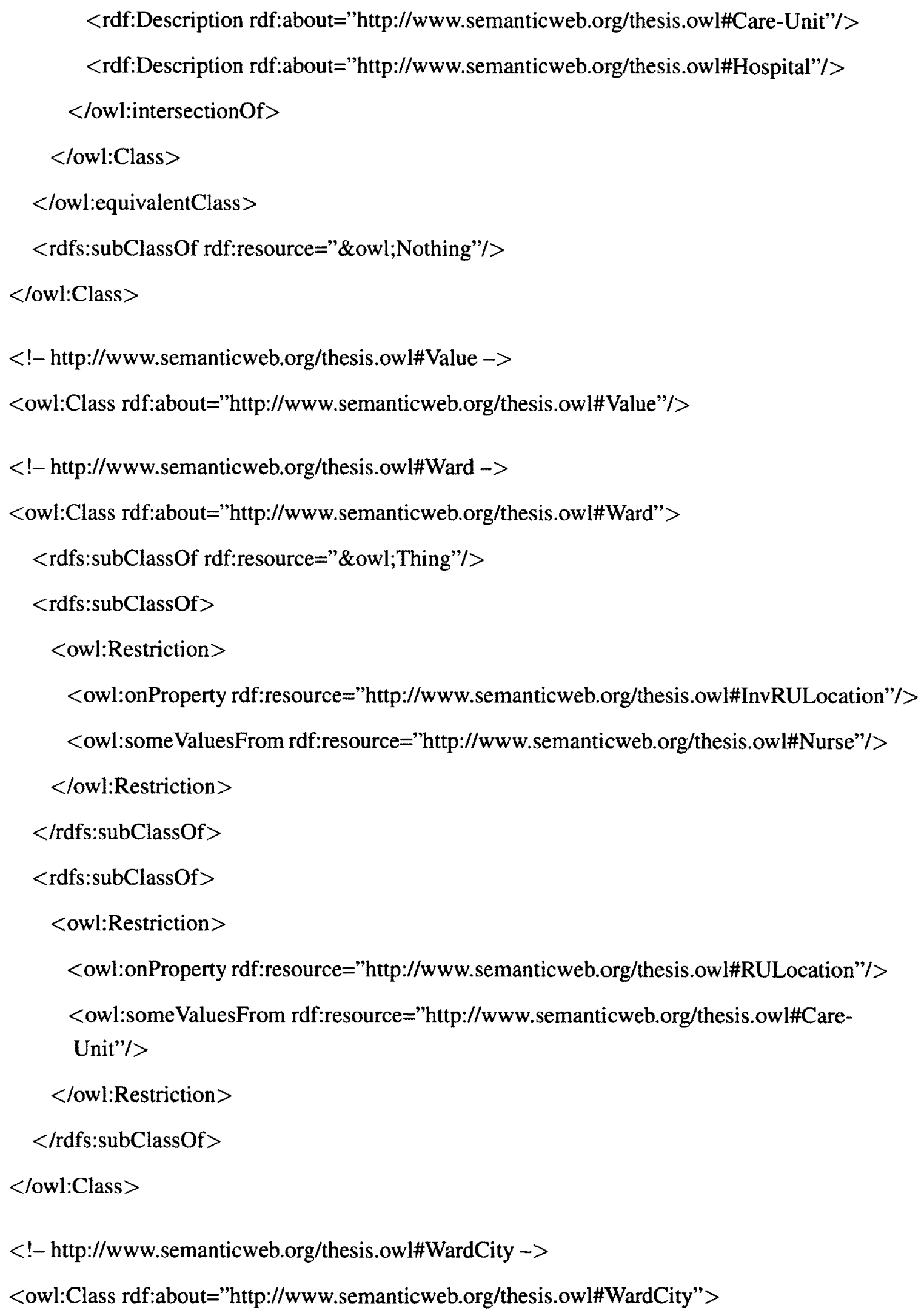




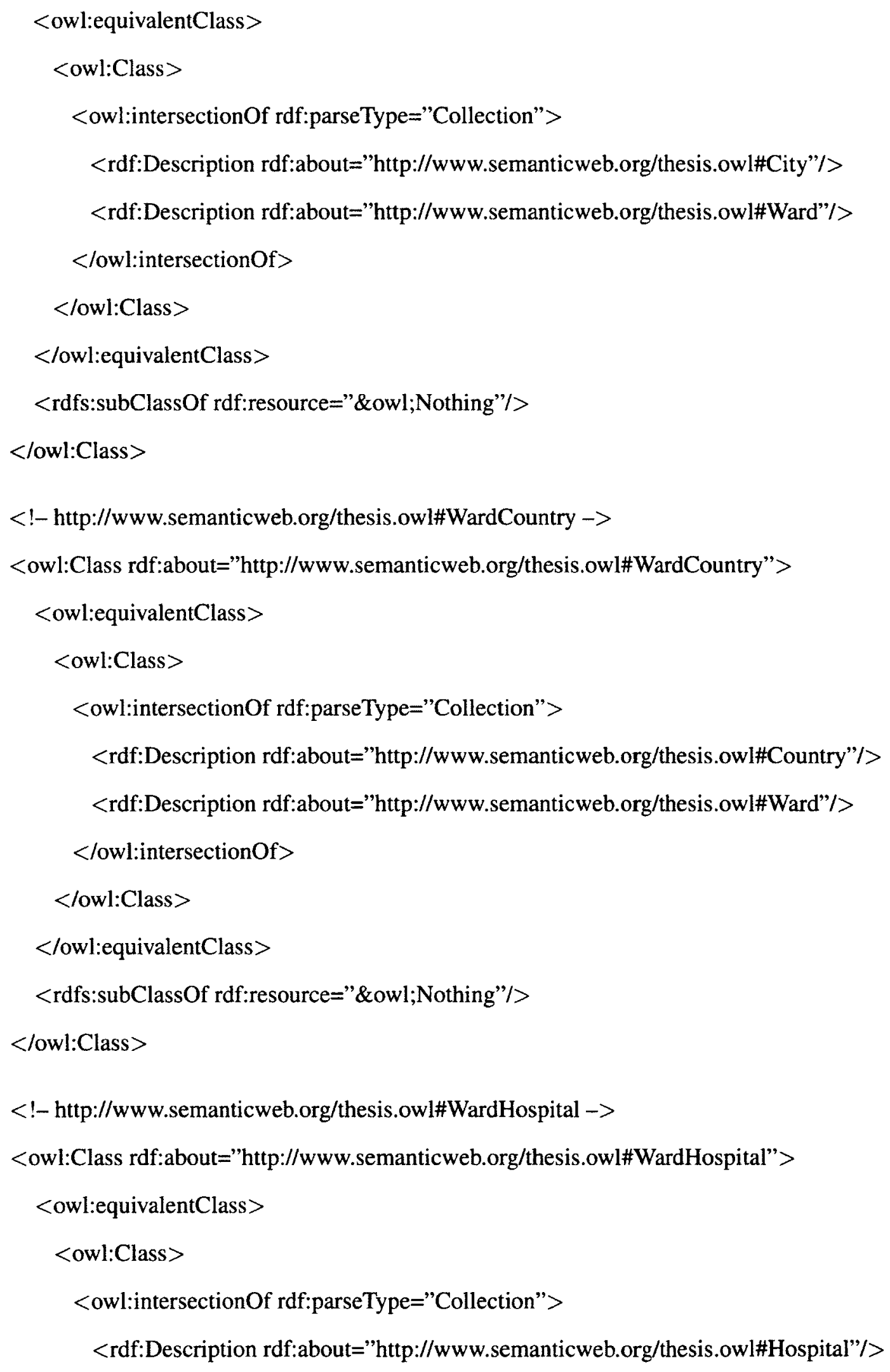




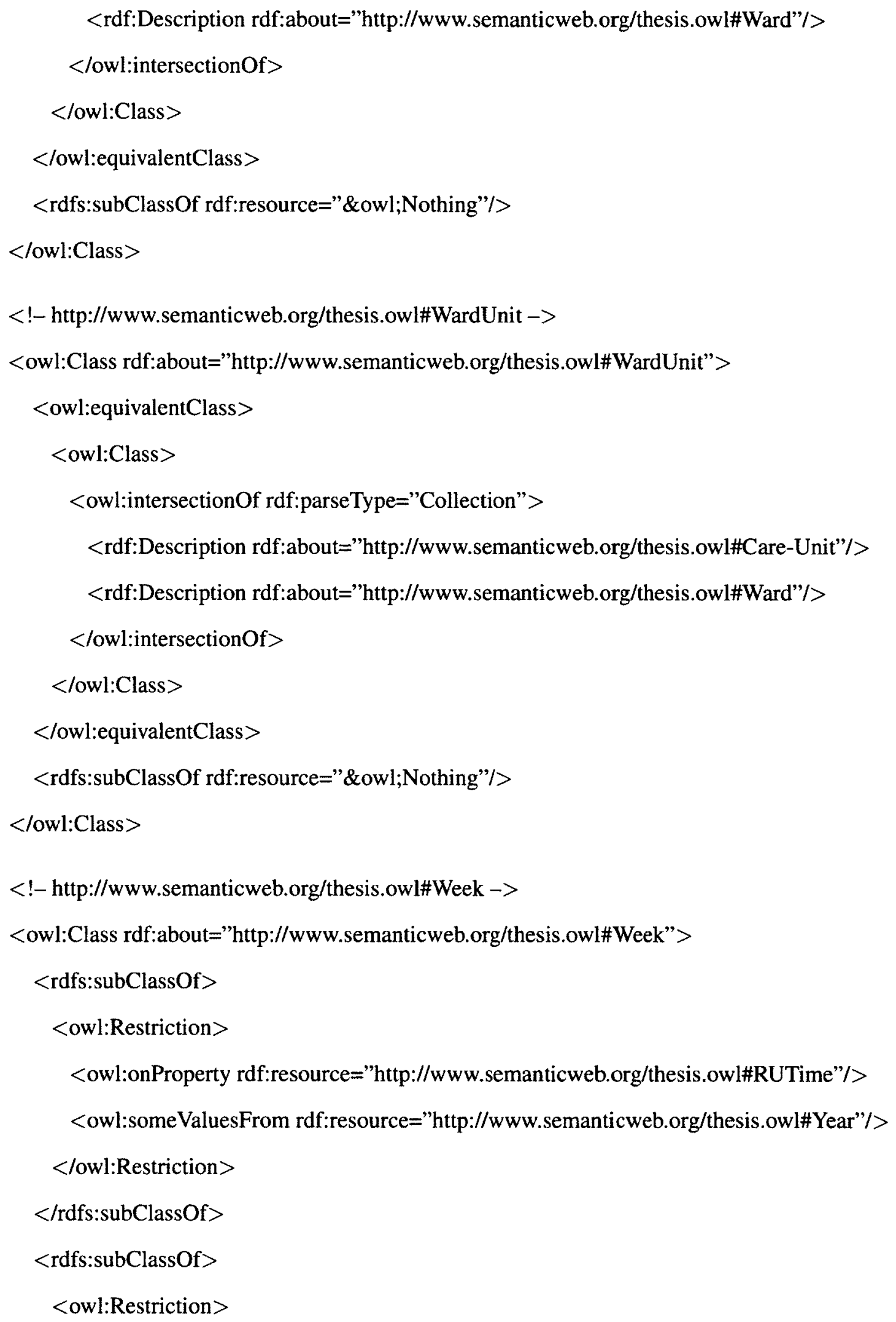




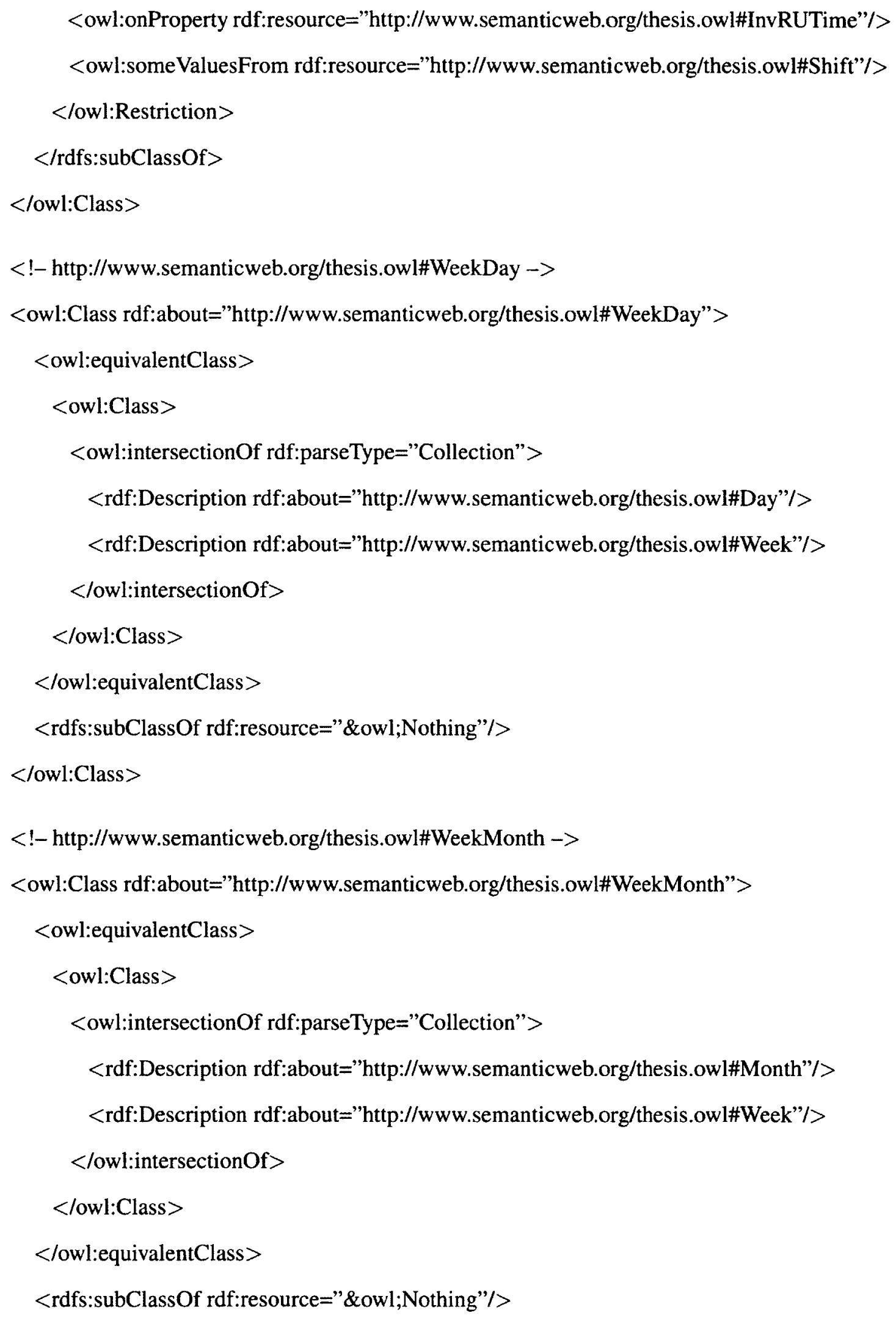




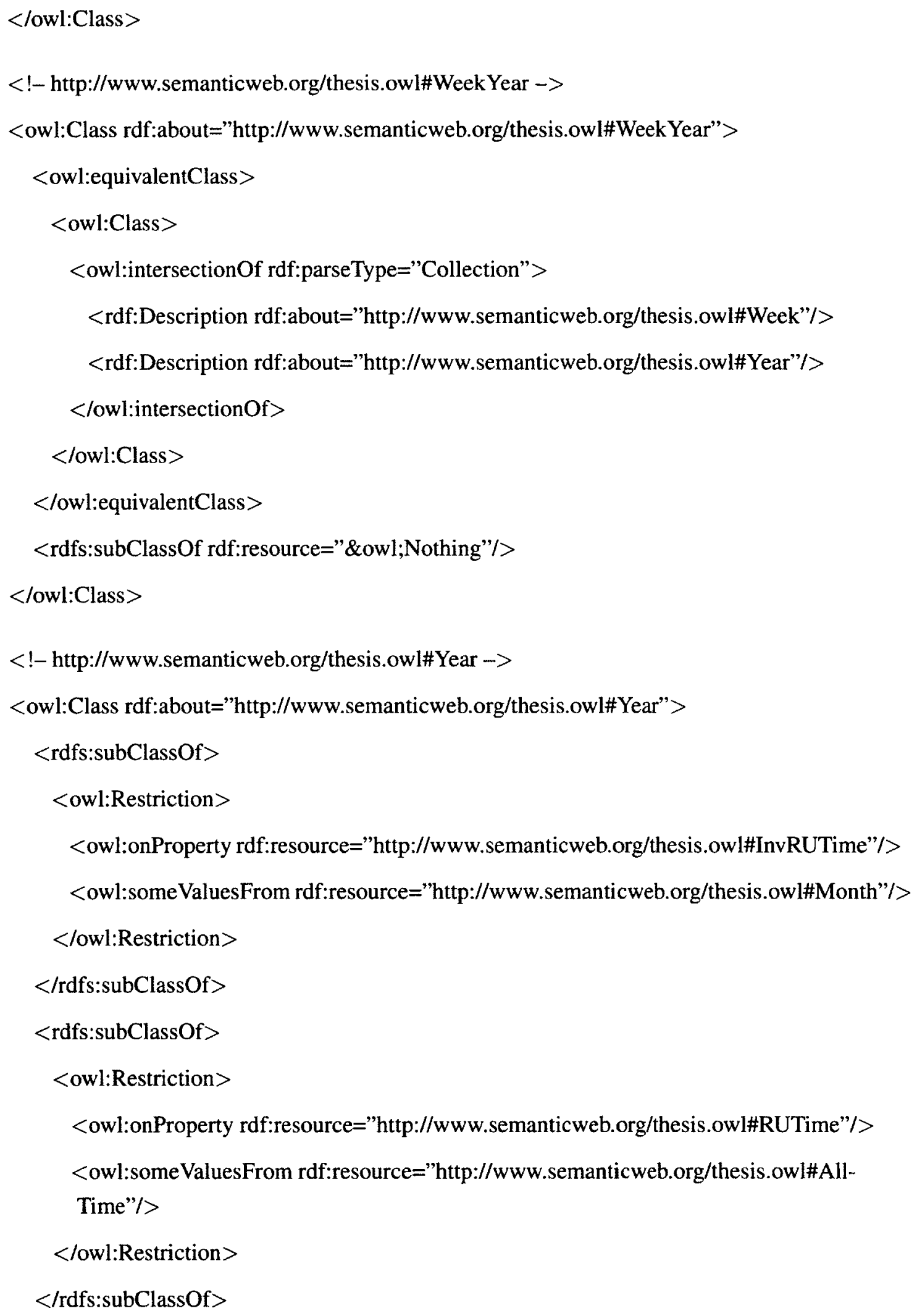




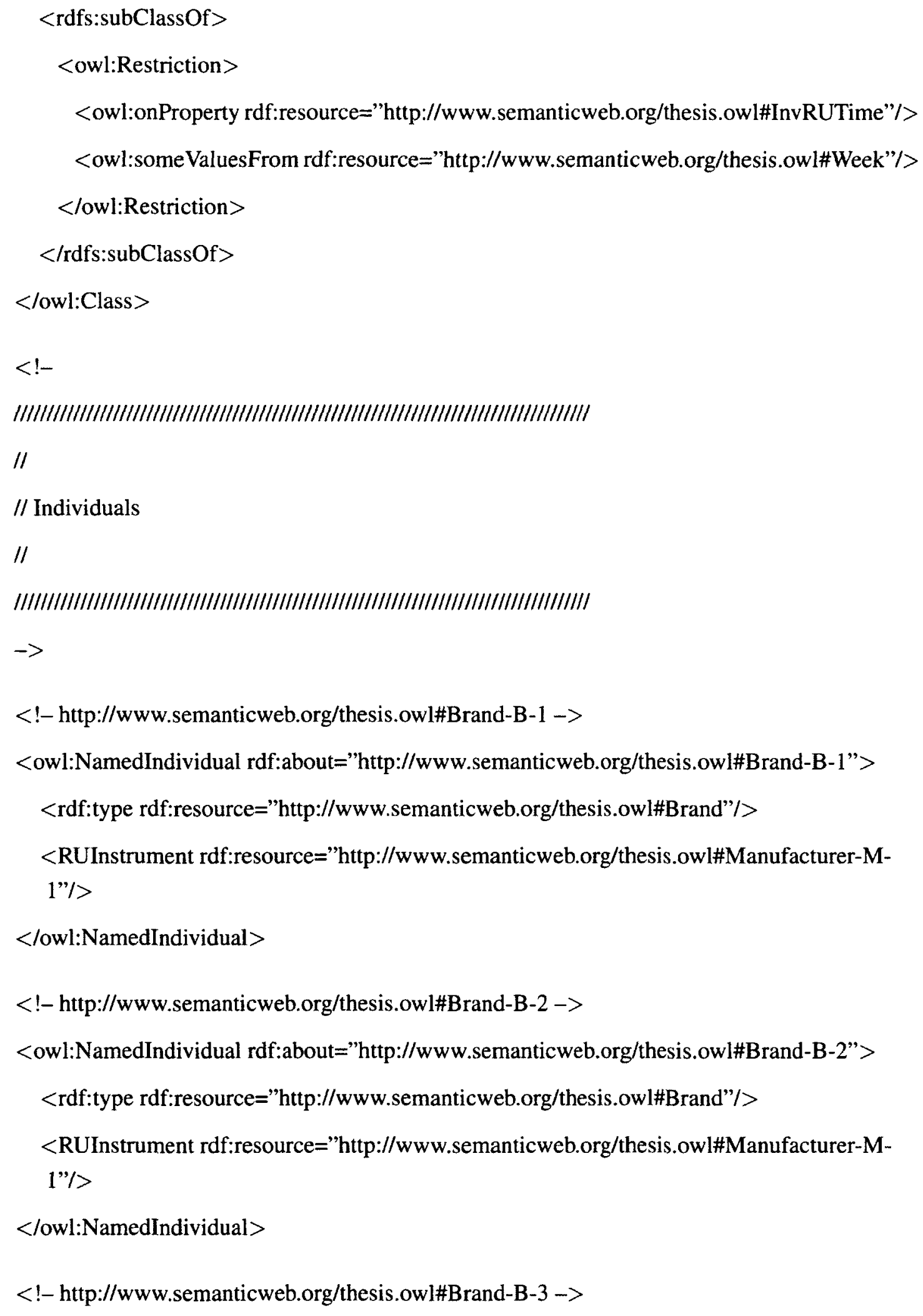


<owl:NamedIndividual rdf:about="http://www.semanticweb.org/thesis.owl\#Brand-B-3"> <rdf:type rdf:resource="http://www.semanticweb.org/thesis.owl\#Brand"/> $<$ RUInstrument rdf:resource="http://www.semanticweb.org/thesis.owl\#Manufacturer-M2"'/>

$<$ lowl:NamedIndividual $>$

$<!-$ http://www.semanticweb.org/thesis.owl\#Brand-B-4 ->

<owl:NamedIndividual rdf:about="http://www.semanticweb.org/thesis.owl\#Brand-B-4">

<rdf:type rdf:resource="http://www.semanticweb.org/thesis.owl\#Brand"/>

$<$ RUInstrument rdf:resource="http://www.semanticweb.org/thesis.owl\#Manufacturer-M3"'/>

$<$ lowl:NamedIndividual $>$

<!- http://www.semanticweb.org/thesis.owl\#Brand-B-5 ->

<owl:NamedIndividual rdf:about="http://www.semanticweb.org/thesis.owl\#Brand-B-5"> <rdf:type rdf:resource="http://www.semanticweb.org/thesis.owl\#Brand"/>

$<$ RUInstrument rdf:resource="http://www.semanticweb.org/thesis.owl\#Manufacturer-M$4 " />$

$<$ lowl:NamedIndividual $>$

$<$ !- http://www.semanticweb.org/thesis.owl\#City-Moscow $\rightarrow>$

<owl:NamedIndividual rdf:about="http://www.semanticweb.org/thesis.owl\#City-Moscow"> <rdf:type rdf:resource="http://www.semanticweb.org/thesis.owl\#City"/>

<RULocation rdf:resource="http://www.semanticweb.org/thesis.owl\#Country-USSR"/> $<$ lowl:NamedIndividual $>$

$<$ !- http://www.semanticweb.org/thesis.owl\#City-Ottawa $->$

<owl:NamedIndividual rdf:about="http://www.semanticweb.org/thesis.owl\#City-Ottawa">

<rdf:type rdf:resource="http://www.semanticweb.org/thesis.owl\#City"/>

<RULocation rdf:resource="http://www.semanticweb.org/thesis.owl\#Country-Ca"/> $</$ owl:NamedIndividual $>$ 
$<$ !- http://www.semanticweb.org/thesis.owl\#Class-BPM ->

<owl:NamedIndividual rdf:about="http://www.semanticweb.org/thesis.owl\#Class-BPM"> <rdf:type rdf:resource="http://www.semanticweb.org/thesis.owl\#Class"/>

</owl:NamedIndividual $>$

$<$ !- http://www.semanticweb.org/thesis.owl\#Class-Stethoscope $\rightarrow>$

<owl:NamedIndividual rdf:about="http://www.semanticweb.org/thesis.owl\#Class-

Stethoscope">

<rdf:type rdf:resource="http://www.semanticweb.org/thesis.owl\#Class"/>

$<$ lowl:NamedIndividual $>$

$<!-$ http://www.semanticweb.org/thesis.owl\#Class-Therm $\rightarrow>$

<owl:NamedIndividual rdf:about="http://www.semanticweb.org/thesis.owl\#Class-Therm"> <rdf:type rdf:resource="http://www.semanticweb.org/thesis.owl\#Class"/>

$<$ /owl:NamedIndividual $>$

$<$ !- http://www.semanticweb.org/thesis.owl\#Country-Ca $->$

<owl:NamedIndividual rdf:about="http://www.semanticweb.org/thesis.owl\#Country-Ca">

<rdf:type rdf:resource="http://www.semanticweb.org/thesis.owl\#Country"/>

$<$ lowl:NamedIndividual $>$

$<$ !- http://www.semanticweb.org/thesis.owl\#Country-USSR ->

<owl:NamedIndividual rdf:about="http://www.semanticweb.org/thesis.owl\#Country-USSR">

<rdf:type rdf:resource="http://www.semanticweb.org/thesis.owl\#Country"/>

$<$ lowl:NamedIndividual $>$

<!- http://www.semanticweb.org/thesis.owl\#Date-5/Sep/2011 ->

<owl:NamedIndividual rdf:about="http://www.semanticweb.org/thesis.owl\#Date$5 / \mathrm{Sep} / 2011 ">$

<rdf:type rdf:resource="http://www.semanticweb.org/thesis.owl\#Day"/>

$<$ lowl:NamedIndividual $>$

$<$ !- http://www.semanticweb.org/thesis.owl\#Date-6/Sep/2011 -> 
<owl:NamedIndividual rdf:about="http://www.semanticweb.org/thesis.owl\#Date6/Sep/2011">

<rdf:type rdf:resource="http://www.semanticweb.org/thesis.owl\#Day"/>

$<$ lowl:NamedIndividual $>$

$<$ !- http://www.semanticweb.org/thesis.owl\#Date-7/Sep/2011 ->

<owl:NamedIndividual rdf:about="http://www.semanticweb.org/thesis.owl\#Date7/Sep/2011">

<rdf:type rdf:resource="http://www.semanticweb.org/thesis.owl\#Day"/>

</owl:NamedIndividual $>$

$<$ !- http://www.semanticweb.org/thesis.owl\#Date-8/Sep/2011 ->

<owl:NamedIndividual rdf:about="http://www.semanticweb.org/thesis.owl\#Date8/Sep/2011">

<rdf:type rdf:resource="http://www.semanticweb.org/thesis.owl\#Day"/>

$<$ /owl:NamedIndividual $>$

$<$ !- http://www.semanticweb.org/thesis.owl\#Day-12/April/2011 ->

<owl:NamedIndividual rdf:about="http://www.semanticweb.org/thesis.owl\#Day-

12/April/2011">

<rdf:type rdf:resource="http://www.semanticweb.org/thesis.owl\#Day"/>

<RUTime rdf:resource="http://www.semanticweb.org/thesis.owl\#Month-April/2011"/>

$<$ lowl:NamedIndividual $>$

<!- http://www.semanticweb.org/thesis.owl\#Day-12/Aug/2010 ->

<owl:NamedIndividual rdf:about="http://www.semanticweb.org/thesis.owl\#Day-

12/Aug/2010">

<rdf:type rdf:resource="http://www.semanticweb.org/thesis.owl\#Day"/>

<RUTime rdf:resource="http://www.semanticweb.org/thesis.owl\#Month-Aug/2010"/>

$<$ lowl:NamedIndividual $>$

<!- http://www.semanticweb.org/thesis.owl\#Day-12/May/2011 -> 
<owl:NamedIndividual rdf:about="http://www.semanticweb.org/thesis.owl\#Day12/May/2011">

<rdf:type rdf:resource="http://www.semanticweb.org/thesis.owl\#Day"/>

<RUTime rdf:resource="http://www.semanticweb.org/thesis.owl\#Month-May/2011"/> $<$ lowl:NamedIndividual $>$

<!- http://www.semanticweb.org/thesis.owl\#Day-12/Sep/2011 ->

<owl:NamedIndividual rdf:about="http://www.semanticweb.org/thesis.owl\#Day12/Sep/2011">

<rdf:type rdf:resource="http://www.semanticweb.org/thesis.owl\#Day"/>

<RUTime rdf:resource="http://www.semanticweb.org/thesis.owl\#Month-Sep/2011"/> $<$ /owl:NamedIndividual $>$

$<!-$ http://www.semanticweb.org/thesis.owl\#Day-16/May/2009 ->

<owl:NamedIndividual rdf:about="http://www.semanticweb.org/thesis.owl\#Day16/May/2009">

<rdf:type rdf:resource="http://www.semanticweb.org/thesis.owl\#Day"/>

$<$ RUTime rdf:resource="http://www.semanticweb.org/thesis.owl\#Month-May/2009"/>

$<$ lowl:NamedIndividual $>$

<!- http://www.semanticweb.org/thesis.owl\#Day-18/April/2009 ->

<owl:NamedIndividual rdf:about="http://www.semanticweb.org/thesis.owl\#Day-

18/April/2009">

<rdf:type rdf:resource="http://www.semanticweb.org/thesis.owl\#Day"/>

<RUTime rdf:resource="http://www.semanticweb.org/thesis.owl\#Month-April/2009"/>

$<$ lowl:NamedIndividual >

<!- http://www.semanticweb.org/thesis.owl\#Day-21/Aug/2010 ->

<owl:NamedIndividual rdf:about="http://www.semanticweb.org/thesis.owl\#Day21/Aug/2010">

< rdf:type rdf:resource="http://www.semanticweb.org/thesis.owl\#Day"/>

<RUTime rdf:resource="http://www.semanticweb.org/thesis.owl\#Month-Aug/2010"/> 
$</$ owl:NamedIndividual $>$

$<$ !- http://www.semanticweb.org/thesis.owl\#Day-24/Aug/2011 ->

<owl:NamedIndividual rdf:about="http://www.semanticweb.org/thesis.owl\#Day24/Aug/2011">

< rdf:type rdf:resource="http://www.semanticweb.org/thesis.owl\#Day"/>

$<$ RUTime rdf:resource="http://www.semanticweb.org/thesis.owl\#Month-Aug/201 l"/> $<$ lowl:NamedIndividual $>$

$<!-$ http://www.semanticweb.org/thesis.owl\#Day-5/Sep/2011 ->

<owl:NamedIndividual rdf:about="http://www.semanticweb.org/thesis.owl\#Day$5 /$ Sep/2011">

<rdf:type rdf:resource="http://www.semanticweb.org/thesis.owl\#Day"/>

$<$ RUTime rdf:resource="http://www.semanticweb.org/thesis.owl\#Month-Sep/2011"/>

$</$ owl:NamedIndividual $>$

$<!-$ http://www.semanticweb.org/thesis.owl\#Day-6/Sep/2011 ->

<owl:NamedIndividual rdf:about="http://www.semanticweb.org/thesis.owl\#Day6/Sep/2011">

$<$ rdf:type rdf:resource="http://www.semanticweb.org/thesis.owl\#Day"/>

$<$ RUTime rdf:resource="http://www.semanticweb.org/thesis.owl\#Month-Sep/2011"/>

$<$ /owl:NamedIndividual $>$

$<!-$ http://www.semanticweb.org/thesis.owl\#Day-7/Aug/2010 ->

<owl:NamedIndividual rdf:about="http://www.semanticweb.org/thesis.owl\#Day-

7/Aug/2010">

$<$ rdf:type rdf:resource="http://www.semanticweb.org/thesis.owl\#Day"/>

$<$ RUTime rdf:resource="http://www.semanticweb.org/thesis.owl\#Month-Aug/2010"/>

$<$ lowl:NamedIndividual $>$

$<$ !- http://www.semanticweb.org/thesis.owl\#Day-7/Sep/2011 ->

<owl:NamedIndividual rdf:about="http://www.semanticweb.org/thesis.owl\#Day7/Sep/2011"> 
<rdf:type rdf:resource="http://www.semanticweb.org/thesis.owl\#Day"/>

<RUTime rdf:resource="http://www.semanticweb.org/thesis.owl\#Month-Sep/2011"/>

$<$ lowl:NamedIndividual $>$

$<$ !- http://www.semanticweb.org/thesis.owl\#Day-8/Aug/2000 ->

<owl:NamedIndividual rdf:about="http://www.semanticweb.org/thesis.owl\#Day-

8/Aug/2000">

<rdf:type rdf:resource="http://www.semanticweb.org/thesis.owl\#Day"/>

$<$ RUTime rdf:resource="http://www.semanticweb.org/thesis.owl\#Month-Aug/2000"/>

</owl:NamedIndividual $>$

<!- http://www.semanticweb.org/thesis.owl\#Day-8/Sep/2011 ->

<owl:NamedIndividual rdf:about="http://www.semanticweb.org/thesis.owl\#Day$8 / \mathrm{Sep} / 2011 ">$

<rdf:type rdf:resource="http://www.semanticweb.org/thesis.owl\#Day"/>

$<$ RUTime rdf:resource="http://www.semanticweb.org/thesis.owl\#Month-Sep/2011"/>

$<$ lowl:NamedIndividual $>$

<!- http://www.semanticweb.org/thesis.owl\#Hospital-H-1 ->

<owl:NamedIndividual rdf:about="http://www.semanticweb.org/thesis.owl\#Hospital-H-1"> <rdf:type rdf:resource="http://www.semanticweb.org/thesis.owl\#Hospital"/>

$<$ RULocation rdf:resource="http://www.semanticweb.org/thesis.owl\#City-Ottawa"/>

$<$ HospitalFounded rdf:resource="http://www.semanticweb.org/thesis.owl\#Day8/Aug/2000"/>

$<$ lowl:NamedIndividual $>$

<!- http://www.semanticweb.org/thesis.owl\#Hospital-H-2 ->

<owl:NamedIndividual rdf:about="http://www.semanticweb.org/thesis.owl\#Hospital-H-2">

<rdf:type rdf:resource="http://www.semanticweb.org/thesis.owl\#Hospital"/>

<RULocation rdf:resource="http://www.semanticweb.org/thesis.owl\#City-Moscow"/>

$<$ HospitalFounded rdf:resource="http://www.semanticweb.org/thesis.owl\#Day-

7/Aug/2010"/> 
$<$ lowl:NamedIndividual $>$

$<!-$ http://www.semanticweb.org/thesis.owl\#Hour-00:45/21/Aug/2010 ->

<owl:NamedIndividual rdf:about="http://www.semanticweb.org/thesis.owl\#Hour-

$00: 45 / 21 /$ Aug/2010">

<rdf:type rdf:resource="http://www.semanticweb.org/thesis.owl\#Hour"/>

<RUTime rdf:resource="http://www.semanticweb.org/thesis.owl\#Day-7/Aug/2010"/>

$<$ RUTime rdf:resource="http://www.semanticweb.org/thesis.owl\#Shift-

Night/Week28/2010"/>

</owl:NamedIndividual $>$

<!- http://www.semanticweb.org/thesis.owl\#Hour-10:18/18/April/2009 ->

<owl:NamedIndividual rdf:about="http://www.semanticweb.org/thesis.owl\#Hour-

10:18/18/April/2009">

< rdf:type rdf:resource="http://www.semanticweb.org/thesis.owl\#Hour"/>

<RUTime rdf:resource="http://www.semanticweb.org/thesis.owl\#Day-18/April/2009"/>

$<$ RUTime rdf:resource="http://www.semanticweb.org/thesis.owl\#Shift-

Morning/Week14/2009"/>

</owl:NamedIndividual $>$

<!- http://www.semanticweb.org/thesis.owl\#Hour-11:10/5/Sep/2011 ->

<owl:NamedIndividual rdf:about="http://www.semanticweb.org/thesis.owl\#Hour-

11:10/5/Sep/2011">

<rdf:type rdf:resource="http://www.semanticweb.org/thesis.owl\#Hour"/>

$</$ owl:NamedIndividual $>$

<!- http://www.semanticweb.org/thesis.owl\#Hour-11:45/5/Sep/2011 ->

<owl:NamedIndividual rdf:about="http://www.semanticweb.org/thesis.owl\#Hour-

$11: 45 / 5 / \mathrm{Sep} / 2011$ ">

<rdf:type rdf:resource="http://www.semanticweb.org/thesis.owl\#Hour"/>

<RUTime rdf:resource="http://www.semanticweb.org/thesis.owl\#Day-5/Sep/2011"/>

<RUTime rdf:resource="http://www.semanticweb.org/thesis.owl\#Shift-

Morning/Week33/2011"/> 
</owl:NamedIndividual $>$

<!- http://www.semanticweb.org/thesis.owl\#Hour-11:45/8/Sep/2011 ->

<owl:NamedIndividual rdf:about="http://www.semanticweb.org/thesis.owl\#Hour-

11:45/8/Sep/2011">

<rdf:type rdf:resource="http://www.semanticweb.org/thesis.owl\#Hour"/>

<RUTime rdf:resource="http://www.semanticweb.org/thesis.owl\#Day-8/Sep/201 1"/>

$<$ RUTime rdf:resource="http://www.semanticweb.org/thesis.owl\#Shift-

Morning/Week/33/2011"/>

$<$ lowl:NamedIndividual $>$

<!- http://www.semanticweb.org/thesis.owl\#Hour-11:50/6/Sep/2011 ->

<owl:NamedIndividual rdf:about="http://www.semanticweb.org/thesis.owl\#Hour-

11:50/6/Sep/2011">

<rdf:type rdf:resource="http://www.semanticweb.org/thesis.owl\#Hour"/>

<RUTime rdf:resource="http://www.semanticweb.org/thesis.owl\#Day-6/Sep/2011"/>

$<$ RUTime rdf:resource="http://www.semanticweb.org/thesis.owl\#Shift-

Morning/Week33/2011"/>

$<$ lowl:NamedIndividual $>$

<!- http://www.semanticweb.org/thesis.owl\#Hour-12:10/5/Sep/2011 ->

<owl:NamedIndividual rdf:about="http://www.semanticweb.org/thesis.owl\#Hour-

$12: 10 / 5 /$ Sep/2011">

< rdf:type rdf:resource="http://www.semanticweb.org/thesis.owl\#Hour"/>

<RUTime rdf:resource="http://www.semanticweb.org/thesis.owl\#Day-5/Sep/2011"/>

<RUTime rdf:resource="http://www.semanticweb.org/thesis.owl\#Shift-

Afternoon/Week33/2011"'/>

$<$ lowl:NamedIndividual $>$

<!- http://www.semanticweb.org/thesis.owl\#Hour-12:15/6/Sep/2011 ->

<owl:NamedIndividual rdf:about="http://www.semanticweb.org/thesis.owl\#Hour-

12:15/6/Sep/2011">

<rdf:type rdf:resource="http://www.semanticweb.org/thesis.owl\#Hour"/> 
<RUTime rdf:resource="http://www.semanticweb.org/thesis.owl\#Day-6/Sep/2011"/> $<$ RUTime rdf:resource="http://www.semanticweb.org/thesis.owl\#ShiftAfternoon/Week33/2011"/>

</owl:NamedIndividual >

<!- http://www.semanticweb.org/thesis.owl\#Hour-12:15/7/Sep/2011 ->

<owl:NamedIndividual rdf:about="http://www.semanticweb.org/thesis.owl\#Hour-

12:15/7/Sep/2011">

<rdf:type rdf:resource="http://www.semanticweb.org/thesis.owl\#Hour"/>

<RUTime rdf:resource="http://www.semanticweb.org/thesis.owl\#Day-7/Sep/2011"/>

$<$ RUTime rdf:resource="http://www.semanticweb.org/thesis.owl\#Shift-

Afternoon/Week33/2011"/>

$<$ lowl:NamedIndividual $>$

<!- http://www.semanticweb.org/thesis.owl\#Hour-14:25/16/May/2009 ->

<owl:NamedIndividual rdf:about="http://www.semanticweb.org/thesis.owl\#Hour-

14:25/16/May/2009">

<rdf:type rdf:resource="http://www.semanticweb.org/thesis.owl\#Hour"/>

$<$ RUTime rdf:resource="http://www.semanticweb.org/thesis.owl\#Day-16/May/2009"/>

$<$ RUTime rdf:resource="http://www.semanticweb.org/thesis.owl\#Shift-

Afternoon/Week18/2009"/>

$<$ lowl:NamedIndividual $>$

<!- http://www.semanticweb.org/thesis.owl\#Hour-15:17/18/April/2009 ->

<owl:NamedIndividual rdf:about="http://www.semanticweb.org/thesis.owl\#Hour-

15:17/18/April/2009">

<rdf:type rdf:resource="http://www.semanticweb.org/thesis.owl\#Hour"/>

<RUTime rdf:resource="http://www.semanticweb.org/thesis.owl\#Day-18/April/2009"/>

$<$ RUTime rdf:resource="http://www.semanticweb.org/thesis.owl\#Shift-

Afternoon/Week14/2009"/>

$<$ lowl:NamedIndividual $>$

$<!-$ http://www.semanticweb.org/thesis.owl\#Hour-21:30/21/Aug/2010 -> 
<owl:NamedIndividual rdf:about="http://www.semanticweb.org/thesis.owl\#Hour-

21:30/21/Aug/2010">

< rdf:type rdf:resource="http://www.semanticweb.org/thesis.owl\#Hour"/>

<RUTime rdf:resource="http://www.semanticweb.org/thesis.owl\#Day-7/Aug/2010"/>

$<$ RUTime rdf:resource="http://www.semanticweb.org/thesis.owl\#Shift-

Night/Week28/2010"/>

$<$ lowl:NamedIndividual $>$

$<$ !- http://www.semanticweb.org/thesis.owl\#Hour-2:30/12/Aug/2010 ->

<owl:NamedIndividual rdf:about="http://www.semanticweb.org/thesis.owl\#Hour-

2:30/12/Aug/2010">

<rdf:type rdf:resource="http://www.semanticweb.org/thesis.owl\#Hour"/>

<RUTime rdf:resource="http://www.semanticweb.org/thesis.owl\#Day-12/Aug/2010"/>

$<$ RUTime rdf:resource="http://www.semanticweb.org/thesis.owl\#Shift-

Night/week30/2010"/>

$</$ owl:NamedIndividual $>$

<!- http://www.semanticweb.org/thesis.owl\#Hour-6:30/12/Aug/2010 ->

<owl:NamedIndividual rdf:about="http://www.semanticweb.org/thesis.owl\#Hour-

6:30/12/Aug/2010">

<rdf:type rdf:resource="http://www.semanticweb.org/thesis.owl\#Hour"/>

<RUTime rdf:resource="http://www.semanticweb.org/thesis.owl\#Day-12/Aug/2010"/>

$<$ RUTime rdf:resource="http://www.semanticweb.org/thesis.owl\#Shift-

morning/week30/2010"/>

$</$ owl:NamedIndividual $>$

$<$ !- http://www.semanticweb.org/thesis.owl\#Hour-8:30/08/Aug/2000 ->

<owl:NamedIndividual rdf:about="http://www.semanticweb.org/thesis.owl\#Hour-

8:30/08/Aug/2000">

<rdf:type rdf:resource="http://www.semanticweb.org/thesis.owl\#Hour"/>

<RUTime rdf:resource="http://www.semanticweb.org/thesis.owl\#Day-8/Aug/2000"/>

$<$ RUTime rdf:resource="http://www.semanticweb.org/thesis.owl\#Shift- 
Morning/Week28/2000"/>

$</$ owl:NamedIndividual $>$

<!- http://www.semanticweb.org/thesis.owl\#Hour-8:30/12/April/2011 ->

<owl:NamedIndividual rdf:about="http://www.semanticweb.org/thesis.owl\#Hour-

8:30/12/April/2011">

<rdf:type rdf:resource="http://www.semanticweb.org/thesis.owl\#Hour"/>

<RUTime rdf:resource="http://www.semanticweb.org/thesis.owl\#Day-12/April/2011"/>

$<$ RUTime rdf:resource="http://www.semanticweb.org/thesis.owl\#Shift-

Morning/Week14/2011"/>

$<$ /owl:NamedIndividual $>$

$<!-$ http://www.semanticweb.org/thesis.owl\#Hour-8:30/12/May/2011 ->

<owl:NamedIndividual rdf:about="http://www.semanticweb.org/thesis.owl\#Hour-

8:30/12/May/2011">

<rdf:type rdf:resource="http://www.semanticweb.org/thesis.owl\#Hour"/>

<RUTime rdf:resource="http://www.semanticweb.org/thesis.owl\#Day-12/May/2011"/>

$<$ RUTime rdf:resource="http://www.semanticweb.org/thesis.owl\#Shift-

Morning/Week 18/2011"/>

$<$ lowl:NamedIndividual >

<!- http://www.semanticweb.org/thesis.owl\#Hour-8:30/12/Sep/2011 ->

<owl:NamedIndividual rdf:about="http://www.semanticweb.org/thesis.owl\#Hour-

$8: 30 / 12 / \mathrm{Sep} / 2011^{\prime \prime}>$

<rdf:type rdf:resource="http://www.semanticweb.org/thesis.owl\#Hour"/>

<RUTime rdf:resource="http://www.semanticweb.org/thesis.owl\#Day-12/Sep/2011"/>

$<$ RUTime rdf:resource="http://www.semanticweb.org/thesis.owl\#Shift-

Morning/Week33/2011"/>

$<$ lowl:NamedIndividual $>$

<!- http://www.semanticweb.org/thesis.owl\#Hour-8:30/24/Aug/2011 ->

<owl:NamedIndividual rdf:about="http://www.semanticweb.org/thesis.owl\#Hour-

8:30/24/Aug/2011" $>$ 
<rdf:type rdf:resource="http://www.semanticweb.org/thesis.owl\#Hour"/>

< RUTime rdf:resource="http://www.semanticweb.org/thesis.owl\#Day-24/Aug/2011"/>

$<$ RUTime rdf:resource="http://www.semanticweb.org/thesis.owl\#Shift-

Morning/Week30/2011'"/>

$<$ /owl:NamedIndividual $>$

<!- http://www.semanticweb.org/thesis.owl\#Hour-9:30/16/May/2009 ->

<owl:NamedIndividual rdf:about="http://www.semanticweb.org/thesis.owl\#Hour-

9:30/16/May/2009">

<rdf:type rdf:resource="http://www.semanticweb.org/thesis.owl\#Hour"/>

<RUTime rdf:resource="http://www.semanticweb.org/thesis.owl\#Day-16/May/2009"/>

$<$ RUTime rdf:resource="http://www.semanticweb.org/thesis.owl\#Shift-

Morning/Week18/2009"/>

$<$ lowl:NamedIndividual $>$

<!- http://www.semanticweb.org/thesis.owl\#Hour-9:30/21/Aug/2010 ->

<owl:NamedIndividual rdf:about="http://www.semanticweb.org/thesis.owl\#Hour-

9:30/21/Aug/2010">

< rdf:type rdf:resource="http://www.semanticweb.org/thesis.owl\#Hour"/>

<RUTime rdf:resource="http://www.semanticweb.org/thesis.owl\#Day-21/Aug/2010"/>

$<$ RUTime rdf:resource="http://www.semanticweb.org/thesis.owl\#Shift-

Morning/Week28/2010"/>

$<$ lowl:NamedIndividual $>$

$<$ !- http://www.semanticweb.org/thesis.owl\#Manufacturer-M-1 ->

<owl:NamedIndividual rdf:about="http://www.semanticweb.org/thesis.owl\#Manufacturer-M-

l">

< rdf:type rdf:resource="http://www.semanticweb.org/thesis.owl\#M1Con"/>

< rdf:type rdf:resource="http://www.semanticweb.org/thesis.owl\#Manufacturer"/>

$<$ lowl:NamedIndividual $>$

<!- http://www.semanticweb.org/thesis.owl\#Manufacturer-M-2 ->

<owl:NamedIndividual rdf:about="http://www.semanticweb.org/thesis.owl\#Manufacturer-M- 


$$
2 ">
$$

< rdf:type rdf:resource="http://www.semanticweb.org/thesis.owl\#Manufacturer"/> $</$ owl:NamedIndividual $>$

<!- http://www.semanticweb.org/thesis.owl\#Manufacturer-M-3 ->

<owl:NamedIndividual rdf:about="http://www.semanticweb.org/thesis.owl\#Manufacturer-M3">

<rdf:type rdf:resource="http://www.semanticweb.org/thesis.owl\#Manufacturer"/>

$<$ lowl:NamedIndividual $>$

$<$ !- http://www.semanticweb.org/thesis.owl\#Manufacturer-M-4 ->

<owl:NamedIndividual rdf:about="http://www.semanticweb.org/thesis.owl\#Manufacturer-M$4 ">$

<rdf:type rdf:resource="http://www.semanticweb.org/thesis.owl\#Manufacturer"/> $<$ lowl:NamedIndividual $>$

$<$ !- http://www.semanticweb.org/thesis.owl\#Model-01-23 ->

<owl:NamedIndividual rdf:about="http://www.semanticweb.org/thesis.owl\#Model-01-23"> <rdf:type rdf:resource="http://www.semanticweb.org/thesis.owl\#Model"/>

$<$ RUInstrument rdf:resource="http://www.semanticweb.org/thesis.owl\#Brand-B-1"/> $<$ RUInstrument rdf:resource="http://www.semanticweb.org/thesis.owl\#Type-Oral"/> $</$ owl:NamedIndividual $>$

$<$ !- http://www.semanticweb.org/thesis.owl\#Model-04-23 ->

<owl:NamedIndividual rdf:about="http://www.semanticweb.org/thesis.owl\#Model-04-23"> <rdf:type rdf:resource="http://www.semanticweb.org/thesis.owl\#Model"/>

<RUInstrument rdf:resource="http://www.semanticweb.org/thesis.owl\#Brand-B-1"/>

$<$ RUInstrument rdf:resource="http://www.semanticweb.org/thesis.owl\#Type-Oral"/> $<$ /owl:NamedIndividual $>$

$<!-$ http://www.semanticweb.org/thesis.owl\#Model-B1-2 ->

<owl:NamedIndividual rdf:about="http://www.semanticweb.org/thesis.owl\#Model-B1-2"> 
< rdf:type rdf:resource="http://www.semanticweb.org/thesis.owl\#Model"/> $<$ RUInstrument rdf:resource="http://www.semanticweb.org/thesis.owl\#Brand-B-3"/> $<$ RUInstrument rdf:resource="http://www.semanticweb.org/thesis.owl\#Type-Arm"/> $</$ owl:NamedIndividual $>$ $<$ !- http://www.semanticweb.org/thesis.owl\#Model-B1-7 -> <owl:NamedIndividual rdf:about="http://www.semanticweb.org/thesis.owl\#Model-B1-7"> <rdf:type rdf:resource="http://www.semanticweb.org/thesis.owl\#Model"/> <RUInstrument rdf:resource="http://www.semanticweb.org/thesis.owl\#Brand-B-3"/> $<$ RUInstrument rdf:resource="http://www.semanticweb.org/thesis.owl\#Type-Arm"/> $<$ lowl:NamedIndividual $>$ <!- http://www.semanticweb.org/thesis.owl\#Model-B2-4 -> <owl:NamedIndividual rdf:about="http://www.semanticweb.org/thesis.owl\#Model-B2-4"> <rdf:type rdf:resource="http://www.semanticweb.org/thesis.owl\#Model"/> <RUInstrument rdf:resource="http://www.semanticweb.org/thesis.owl\#Brand-B-4"/> <RUInstrument rdf:resource="http://www.semanticweb.org/thesis.owl\#Type-Finger"/> $<$ lowl:NamedIndividual $>$ <!- http://www.semanticweb.org/thesis.owl\#Model-B3-5 -> <owl:NamedIndividual rdf:about="http://www.semanticweb.org/thesis.owl\#Model-B3-5"> <rdf:type rdf:resource="http://www.semanticweb.org/thesis.owl\#Model"/> <RUInstrument rdf:resource="http://www.semanticweb.org/thesis.owl\#Brand-B-4"/> $<$ RUInstrument rdf:resource="http://www.semanticweb.org/thesis.owl\#Type-Finger"/> $<$ /owl:NamedIndividual $>$

<!- http://www.semanticweb.org/thesis.owl\#Model-S-37 -> <owl:NamedIndividual rdf:about="http://www.semanticweb.org/thesis.owl\#Model-S-37"> < rdf:type rdf:resource="http://www.semanticweb.org/thesis.owl\#Model"/> <RUInstrument rdf:resource="http://www.semanticweb.org/thesis.owl\#Brand-B-5"/> $<$ RUInstrument rdf:resource="http://www.semanticweb.org/thesis.owl\#Type-Electronic"/> 
$<$ /owl:NamedIndividual $>$

$<$ !- http://www.semanticweb.org/thesis.owl\#Model-S-48 ->

<owl:NamedIndividual rdf:about="http://www.semanticweb.org/thesis.owl\#Model-S-48">

<rdf:type rdf:resource="http://www.semanticweb.org/thesis.owl\#Model"/>

$<$ RUInstrument rdf:resource="http://www.semanticweb.org/thesis.owl\#Brand-B-5"/>

< RUInstrument rdf:resource="http://www.semanticweb.org/thesis.owl\#Type-Electronic"/>

$</$ owl:NamedIndividual $>$

<!- http://www.semanticweb.org/thesis.owl\#Model-T-33 ->

<owl:NamedIndividual rdf:about="http://www.semanticweb.org/thesis.owl\#Model-T-33">

<rdf:type rdf:resource="http://www.semanticweb.org/thesis.owl\#Model"/>

$<$ RUInstrument rdf:resource="http://www.semanticweb.org/thesis.owl\#Brand-B-2"/>

$<$ RUInstrument rdf:resource="http://www.semanticweb.org/thesis.owl\#Type-Tymp"/>

$</$ owl:NamedIndividual $>$

<!- http://www.semanticweb.org/thesis.owl\#Model-T-42 ->

<owl:NamedIndividual rdf:about="http://www.semanticweb.org/thesis.owl\#Model-T-42">

< rdf:type rdf:resource="http://www.semanticweb.org/thesis.owl\#Model"/>

<RUInstrument rdf:resource="http://www.semanticweb.org/thesis.owl\#Brand-B-2"/>

<RUInstrument rdf:resource="http://www.semanticweb.org/thesis.owl\#Type-Tymp"/>

$<$ lowl:NamedIndividual $>$

$<$ !- http://www.semanticweb.org/thesis.owl\#Month-April/2009 ->

<owl:NamedIndividual rdf:about="http://www.semanticweb.org/thesis.owl\#MonthApril/2009">

<rdf:type rdf:resource="http://www.semanticweb.org/thesis.owl\#Month"/>

<RUTime rdf:resource="http://www.semanticweb.org/thesis.owl\#Year-2009"/>

$<$ lowl:NamedIndividual $>$

<!- http://www.semanticweb.org/thesis.owl\#Month-April/2011 ->

<owl:NamedIndividual rdf:about="http://www.semanticweb.org/thesis.owl\#Month- 
April/2011">

<rdf:type rdf:resource="http://www.semanticweb.org/thesis.owl\#Month"/>

<RUTime rdf:resource="http://www.semanticweb.org/thesis.owl\#Year-2011"/>

$<$ lowl:NamedIndividual $>$

<!- http://www.semanticweb.org/thesis.owl\#Month-Aug/2000 ->

<owl:NamedIndividual rdf:about="http://www.semanticweb.org/thesis.owl\#MonthAug/2000">

<rdf:type rdf:resource="http://www.semanticweb.org/thesis.owl\#Month"/>

<RUTime rdf:resource="http://www.semanticweb.org/thesis.owl\#Year-2000"/>

$<$ /owl:NamedIndividual $>$

$<$ !- http://www.semanticweb.org/thesis.owl\#Month-Aug/2010 ->

<owl:NamedIndividual rdf:about="http://www.semanticweb.org/thesis.owl\#MonthAug/2010">

<rdf:type rdf:resource="http://www.semanticweb.org/thesis.owl\#Month"/>

<RUTime rdf:resource="http://www.semanticweb.org/thesis.owl\#Year-2010"/>

$<$ lowl:NamedIndividual $>$

<!- http://www.semanticweb.org/thesis.owl\#Month-Aug/2011 ->

<owl:NamedIndividual rdf:about="http://www.semanticweb.org/thesis.owl\#MonthAug/2011">

<rdf:type rdf:resource="http://www.semanticweb.org/thesis.owl\#Month"/>

<RUTime rdf:resource="http://www.semanticweb.org/thesis.owl\#Year-2011"/>

$<$ lowl:NamedIndividual $>$

<!- http://www.semanticweb.org/thesis.owl\#Month-May/2009 ->

<owl:NamedIndividual rdf:about="http://www.semanticweb.org/thesis.owl\#MonthMay/2009">

<rdf:type rdf:resource="http://www.semanticweb.org/thesis.owl\#Month"/>

$<$ RUTime rdf:resource="http://www.semanticweb.org/thesis.owl\#Year-2009"/>

$</$ owl:NamedIndividual $>$ 
<!- http://www.semanticweb.org/thesis.owl\#Month-May/2011 ->

<owl:NamedIndividual rdf:about="http://www.semanticweb.org/thesis.owl\#MonthMay/2011">

<rdf:type rdf:resource="http://www.semanticweb.org/thesis.owl\#Month"/>

<RUTime rdf:resource="http://www.semanticweb.org/thesis.owl\#Year-2011"/>

$</$ owl:NamedIndividual $>$

$<$ !- http://www.semanticweb.org/thesis.owl\#Month-Sep/2011 ->

<owl:NamedIndividual rdf:about="http://www.semanticweb.org/thesis.owl\#Month$\mathrm{Sep} / 2011 ">$

<rdf:type rdf:resource="http://www.semanticweb.org/thesis.owl\#Month"/>

< RUTime rdf:resource="http://www.semanticweb.org/thesis.owl\#Year-2011"/>

$</$ owl:NamedIndividual $>$

$<$ !- http://www.semanticweb.org/thesis.owl\#Nurse-Agatha $->$

<owl:NamedIndividual rdf:about="http://www.semanticweb.org/thesis.owl\#Nurse-Agatha">

<rdf:type rdf:resource="http://www.semanticweb.org/thesis.owl\#Nurse"/>

<RULocation rdf:resource="http://www.semanticweb.org/thesis.owl\#Ward-W-4"/>

$<$ lowl:NamedIndividual $>$

<!- http://www.semanticweb.org/thesis.owl\#Nurse-Anna $->$

<owl:NamedIndividual rdf:about="http://www.semanticweb.org/thesis.owl\#Nurse-Anna">

<rdf:type rdf:resource="http://www.semanticweb.org/thesis.owl\#Nurse"/>

$<$ RULocation rdf:resource="http://www.semanticweb.org/thesis.owl\#Ward-W-5"/>

$<$ /owl:NamedIndividual $>$

$<$ !- http://www.semanticweb.org/thesis.owl\#Nurse-Cathy $\rightarrow>$

<owl:NamedIndividual rdf:about="http://www.semanticweb.org/thesis.owl\#Nurse-Cathy">

<rdf:type rdf:resource="http://www.semanticweb.org/thesis.owl\#Nurse"/>

<RULocation rdf:resource="http://www.semanticweb.org/thesis.owl\#Ward-W-1"/>

$<$ /owl:NamedIndividual $>$ 
$<$ !- http://www.semanticweb.org/thesis.owl\#Nurse-Jane $->$

<owl:NamedIndividual rdf:about="http://www.semanticweb.org/thesis.owl\#Nurse-Jane"> <rdf:type rdf:resource="http://www.semanticweb.org/thesis.owl\#Nurse"/>

<RULocation rdf:resource="http://www.semanticweb.org/thesis.owl\#Ward-W-2"/> $<$ lowl:NamedIndividual $>$

<!- http://www.semanticweb.org/thesis.owl\#Nurse-Juan ->

<owl:NamedIndividual rdf:about="http://www.semanticweb.org/thesis.owl\#Nurse-Juan"> <rdf:type rdf:resource="http://www.semanticweb.org/thesis.owl\#Nurse"/>

$<$ RULocation rdf:resource="http://www.semanticweb.org/thesis.owl\#Ward-W-3"/> $<$ lowl:NamedIndividual >

$<$ !- http://www.semanticweb.org/thesis.owl\#Nurse-Lauren $\rightarrow>$

<owl:NamedIndividual rdf:about="http://www.semanticweb.org/thesis.owl\#Nurse-Lauren"> < rdf:type rdf:resource="http://www.semanticweb.org/thesis.owl\#Nurse"/>

<RULocation rdf:resource="http://www.semanticweb.org/thesis.owl\#Ward-W-4"/> $</$ owl:NamedIndividual $>$

<!- http://www.semanticweb.org/thesis.owl\#Nurse-Lori -> <owl:NamedIndividual rdf:about="http://www.semanticweb.org/thesis.owl\#Nurse-Lori"> < rdf:type rdf:resource="http://www.semanticweb.org/thesis.owl\#Nurse"/>

<RULocation rdf:resource="http://www.semanticweb.org/thesis.owl\#Ward-W-3"/> $<$ lowl:NamedIndividual $>$

$<$ !- http://www.semanticweb.org/thesis.owl\#Nurse-Mary $\rightarrow>$

<owl:NamedIndividual rdf:about="http://www.semanticweb.org/thesis.owl\#Nurse-Mary"> <rdf:type rdf:resource="http://www.semanticweb.org/thesis.owl\#Nurse"/>

<RULocation rdf:resource="http://www.semanticweb.org/thesis.owl\#Ward-W-4"/> $<$ lowl:NamedIndividual $>$

$<$ !- http://www.semanticweb.org/thesis.owl\#Nurse-Sarah $\rightarrow>$

<owl:NamedIndividual rdf:about="http://www.semanticweb.org/thesis.owl\#Nurse-Sarah"> 
<rdf:type rdf:resource="http://www.semanticweb.org/thesis.owl\#Nurse"/>

<RULocation rdf:resource="http://www.semanticweb.org/thesis.owl\#Ward-W-5"/> $<$ lowl:NamedIndividual >

$<!-$ http://www.semanticweb.org/thesis.owl\#Nurse-Suzan $\rightarrow>$

<owl:NamedIndividual rdf:about="http://www.semanticweb.org/thesis.owl\#Nurse-Suzan">

<rdf:type rdf:resource="http://www.semanticweb.org/thesis.owl\#Nurse"/>

< RULocation rdf:resource="http://www.semanticweb.org/thesis.owl\#Ward-W-1"/>

$<$ lowl:NamedIndividual >

$<$ !- http://www.semanticweb.org/thesis.owl\#PW/Lou

<owl:NamedIndividual rdf:about="http://www.semanticweb.org/thesis.owl\#PW/Lou

<rdf:type rdf:resource="http://www.semanticweb.org/thesis.owl\#PW"/>

<PWHour rdf:resource="http://www.semanticweb.org/thesis.owl\#Hour11:10/5/Sep/2011"/>

$<$ PWPatient rdf:resource="http://www.semanticweb.org/thesis.owl\#Patient-Lou <PWValue rdf:resource="http://www.semanticweb.org/thesis.owl\#Value-37.9"/>

<PWWard rdf:resource="http://www.semanticweb.org/thesis.owl\#Ward-W-2"/>

$<$ lowl:NamedIndividual $>$

<!- http://www.semanticweb.org/thesis.owl\#PW/Tom

<owl:NamedIndividual rdf:about="http://www.semanticweb.org/thesis.owl\#PW/Tom

<rdf:type rdf:resource="http://www.semanticweb.org/thesis.owl\#PW"/>

$<$ PWHour rdf:resource="http://www.semanticweb.org/thesis.owl\#Hour-

$11: 45 / 8 / \mathrm{Sep} / 2011 " />$

<PWPatient rdf:resource="http://www.semanticweb.org/thesis.owl\#Patient-Tom

<PWValue rdf:resource="http://www.semanticweb.org/thesis.owl\#Value-110/70"/>

<PWWard rdf:resource="http://www.semanticweb.org/thesis.owl\#Ward-W-3"/>

$</$ owl:NamedIndividual $>$

$<$ !- http://www.semanticweb.org/thesis.owl\#PW/Tom 
<owl:NamedIndividual rdf:about="http://www.semanticweb.org/thesis.owl\#PW/Tom < rdf:type rdf:resource="http://www.semanticweb.org/thesis.owl\#PW"/> $<$ PWHour rdf:resource="http://www.semanticweb.org/thesis.owl\#Hour$12: 15 / 7 / \mathrm{Sep} / 2011 " />$

<PWPatient rdf:resource="http://www.semanticweb.org/thesis.owl\#Patient-Tom <PWValue rdf:resource="http://www.semanticweb.org/thesis.owl\#Value-38.0"/> <PWWard rdf:resource="http://www.semanticweb.org/thesis.owl\#Ward-W-1"/> $<$ lowl:NamedIndividual $>$

$<!-$ http://www.semanticweb.org/thesis.owl\#PW/Tom

<owl:NamedIndividual rdf:about="http://www.semanticweb.org/thesis.owl\#PW/Tom <rdf:type rdf:resource="http://www.semanticweb.org/thesis.owl\#PW"/> $<$ PWHour rdf:resource="http://www.semanticweb.org/thesis.owl\#Hour11:50/6/Sep/2011"/>

<PWPatient rdf:resource="http://www.semanticweb.org/thesis.owl\#Patient-Tom <PWValue rdf:resource="http://www.semanticweb.org/thesis.owl\#Value-38.1"/> <PWWard rdf:resource="http://www.semanticweb.org/thesis.owl\#Ward-W-1"/> $<$ lowl:NamedIndividual $>$

<!- http://www.semanticweb.org/thesis.owl\#PW/Tom

<owl:NamedIndividual rdf:about="http://www.semanticweb.org/thesis.owl\#PW/Tom <rdf:type rdf:resource="http://www.semanticweb.org/thesis.owl\#PW"/> <PWHour rdf:resource="http://www.semanticweb.org/thesis.owl\#Hour$12: 10 / 5 / \mathrm{Sep} / 2011 " />$ <PWPatient rdf:resource="http://www.semanticweb.org/thesis.owl\#Patient-Tom <PWValue rdf:resource="http://www.semanticweb.org/thesis.owl\#Value-38.2"/> <PWWard rdf:resource="http://www.semanticweb.org/thesis.owl\#Ward-W-1"/> $<$ lowl:NamedIndividual $>$ <!- http://www.semanticweb.org/thesis.owl\#PW/Tom <owl:NamedIndividual rdf:about="http://www.semanticweb.org/thesis.owl\#PW/Tom 
<rdf:type rdf:resource="http://www.semanticweb.org/thesis.owl\#PW"/> <PWHour rdf:resource="http://www.semanticweb.org/thesis.owl\#Hour$11: 45 / 5 / \operatorname{Sep} / 2011 \% />$

$<$ PWPatient rdf:resource="http://www.semanticweb.org/thesis.owl\#Patient-Tom <PWValue rdf:resource="http://www.semanticweb.org/thesis.owl\#Value-38.5"/> <PWWard rdf:resource="http://www.semanticweb.org/thesis.owl\#Ward-W-1"/> </owl:NamedIndividual >

$<$ !- http://www.semanticweb.org/thesis.owl\#Patient-Lou <owl:NamedIndividual rdf:about="http://www.semanticweb.org/thesis.owl\#Patient-Lou <rdf:type rdf:resource="http://www.semanticweb.org/thesis.owl\#Patient"/> $<$ lowl:NamedIndividual $>$ $<!-$ http://www.semanticweb.org/thesis.owl\#Patient-Tom <owl:NamedIndividual rdf:about="http://www.semanticweb.org/thesis.owl\#Patient-Tom <rdf:type rdf:resource="http://www.semanticweb.org/thesis.owl\#Patient"/> $<$ lowl:NamedIndividual $>$ <!- http://www.semanticweb.org/thesis.owl\#Shift-Afternoon/Week14/2009 -> <owl:NamedIndividual rdf:about="http://www.semanticweb.org/thesis.owl\#ShiftAfternoon/Week14/2009"> <rdf:type rdf:resource="http://www.semanticweb.org/thesis.owl\#Shift"/> < RUTime rdf:resource="http://www.semanticweb.org/thesis.owl\#Week-Week14/2009"/> $<$ lowl:NamedIndividual $>$ <!- http://www.semanticweb.org/thesis.owl\#Shift-Afternoon/Week18/2009 -> <owl:NamedIndividual rdf:about="http://www.semanticweb.org/thesis.owl\#ShiftAfternoon/Week18/2009"> <rdf:type rdf:resource="http://www.semanticweb.org/thesis.owl\#Shift"/> < RUTime rdf:resource="http://www.semanticweb.org/thesis.owl\#Week-Week18/2009"/> $</$ owl:NamedIndividual $>$ 
<!- http://www.semanticweb.org/thesis.owl\#Shift-Afternoon/Week33/2011 ->

<owl:NamedIndividual rdf:about="http://www.semanticweb.org/thesis.owl\#Shift-

Afternoon/Week33/2011">

<rdf:type rdf:resource="http://www.semanticweb.org/thesis.owl\#Shift"/>

$<$ RUTime rdf:resource="http://www.semanticweb.org/thesis.owl\#Week-Week/33/2011"/>

$<$ /owl:NamedIndividual $>$

<!- http://www.semanticweb.org/thesis.owl\#Shift-Morning/Week/33/2011 ->

<owl:NamedIndividual rdf:about="http://www.semanticweb.org/thesis.owl\#Shift-

Morning/Week/33/2011">

<rdf:type rdf:resource="http://www.semanticweb.org/thesis.owl\#Shift"/>

$<$ RUTime rdf:resource="http://www.semanticweb.org/thesis.owl\#Week-Week/33/2011"/>

$<$ lowl:NamedIndividual $>$

<!- http://www.semanticweb.org/thesis.owl\#Shift-Morning/Week14/2009 ->

<owl:NamedIndividual rdf:about="http://www.semanticweb.org/thesis.owl\#Shift-

Morning/Week14/2009">

<rdf:type rdf:resource="http://www.semanticweb.org/thesis.owl\#Shift"/>

$<$ RUTime rdf:resource="http://www.semanticweb.org/thesis.owl\#Week-Week14/2009"/>

$</$ owl:NamedIndividual $>$

<!- http://www.semanticweb.org/thesis.owl\#Shift-Morning/Week14/2011 ->

<owl:NamedIndividual rdf:about="http://www.semanticweb.org/thesis.owl\#Shift-

Morning/Week14/2011">

<rdf:type rdf:resource="http://www.semanticweb.org/thesis.owl\#Shift"/>

< RUTime rdf:resource="http://www.semanticweb.org/thesis.owl\#Week-Week14/2011"/>

</owl:NamedIndividual $>$

<!- http://www.semanticweb.org/thesis.owl\#Shift-Morning/Week18/2009 ->

<owl:NamedIndividual rdf:about="http://www.semanticweb.org/thesis.owl\#Shift-

Morning/Week18/2009">

< rdf:type rdf:resource="http://www.semanticweb.org/thesis.owl\#Shift"/> 
<RUTime rdf:resource="http://www.semanticweb.org/thesis.owl\#Week-Week18/2009"/> $<$ lowl:NamedIndividual $>$

<!- http://www.semanticweb.org/thesis.owl\#Shift-Morning/Week18/2011 ->

<owl:NamedIndividual rdf:about="http://www.semanticweb.org/thesis.owl\#ShiftMorning/Week 18/2011">

<rdf:type rdf:resource="http://www.semanticweb.org/thesis.owl\#Shift"/>

<RUTime rdf:resource="http://www.semanticweb.org/thesis.owl\#Week-Week18/2011"/>

$</$ owl:NamedIndividual $>$

$<$ !- http://www.semanticweb.org/thesis.owl\#Shift-Morning/Week28/2000 ->

<owl:NamedIndividual rdf:about="http://www.semanticweb.org/thesis.owl\#Shift-

Morning/Week28/2000">

<rdf:type rdf:resource="http://www.semanticweb.org/thesis.owl\#Shift"/>

<RUTime rdf:resource="http://www.semanticweb.org/thesis.owl\#Week-Week28/2000"/>

$<$ lowl:NamedIndividual $>$

<!- http://www.semanticweb.org/thesis.owl\#Shift-Morning/Week28/2010 ->

<owl:NamedIndividual rdf:about="http://www.semanticweb.org/thesis.owl\#Shift-

Morning/Week28/2010">

<rdf:type rdf:resource="http://www.semanticweb.org/thesis.owl\#Shift"/>

$<$ RUTime rdf:resource="http://www.semanticweb.org/thesis.owl\#Week-Week28/2010"/>

$<$ lowl:NamedIndividual $>$

$<$ !- http://www.semanticweb.org/thesis.owl\#Shift-Morning/Week30/2011 ->

<owl:NamedIndividual rdf:about="http://www.semanticweb.org/thesis.owl\#Shift-

Morning/Week30/2011">

<rdf:type rdf:resource="http://www.semanticweb.org/thesis.owl\#Shift"/>

<RUTime rdf:resource="http://www.semanticweb.org/thesis.owl\#Week-Week30/2011"/>

$</$ owl:NamedIndividual $>$

$<!-$ http://www.semanticweb.org/thesis.owl\#Shift-Morning/Week33/2011 ->

<owl:NamedIndividual rdf:about="http://www.semanticweb.org/thesis.owl\#Shift- 


\section{Morning/Week33/2011">}

<rdf:type rdf:resource="http://www.semanticweb.org/thesis.owl\#Shift"/>

<RUTime rdf:resource="http://www.semanticweb.org/thesis.owl\#Week-Week/33/2011"/>

<RUTime rdf:resource="http://www.semanticweb.org/thesis.owl\#Week-Week33/2011"/>

$<$ lowl:NamedIndividual $>$

<!- http://www.semanticweb.org/thesis.owl\#Shift-Night/Week28/2010 ->

<owl:NamedIndividual rdf:about="http://www.semanticweb.org/thesis.owl\#Shift-

Night/Week28/2010">

<rdf:type rdf:resource="http://www.semanticweb.org/thesis.owl\#Shift"/>

< RUTime rdf:resource="http://www.semanticweb.org/thesis.owl\#Week-Week28/2010"/>

$<$ lowl:NamedIndividual $>$

<!- http://www.semanticweb.org/thesis.owl\#Shift-Night/week30/2010 ->

<owl:NamedIndividual rdf:about="http://www.semanticweb.org/thesis.owl\#Shift-

Night/week30/2010">

< rdf:type rdf:resource="http://www.semanticweb.org/thesis.owl\#Shift"/>

<RUTime rdf:resource="http://www.semanticweb.org/thesis.owl\#Week-week30/2010"/>

<lowl:NamedIndividual $>$

<!- http://www.semanticweb.org/thesis.owl\#Shift-morning/week30/2010->

<owl:NamedIndividual rdf:about="http://www.semanticweb.org/thesis.owl\#Shift-

morning/week30/2010">

<rdf:type rdf:resource="http://www.semanticweb.org/thesis.owl\#Shift"/>

<RUTime rdf:resource="http://www.semanticweb.org/thesis.owl\#Week-week30/2010"/>

$<$ lowl:NamedIndividual $>$

$<$ !- http://www.semanticweb.org/thesis.owl\#Type-Arm ->

<owl:NamedIndividual rdf:about="http://www.semanticweb.org/thesis.owl\#Type-Arm">

<rdf:type rdf:resource="http://www.semanticweb.org/thesis.owl\#Type"/>

$<$ RUInstrument rdf:resource="http://www.semanticweb.org/thesis.owl\#Class-BPM"/>

$</$ owl:NamedIndividual $>$ 
$<$ !- http://www.semanticweb.org/thesis.owl\#Type-Electronic $\rightarrow>$

<owl:NamedIndividual rdf:about="http://www.semanticweb.org/thesis.owl\#Type-

Electronic">

<rdf:type rdf:resource="http://www.semanticweb.org/thesis.owl\#Type"/>

$<$ RUInstrument rdf:resource="http://www.semanticweb.org/thesis.owl\#Class-

Stethoscope"/>

</owl:NamedIndividual >

$<!-$ http://www.semanticweb.org/thesis.owl\#Type-Finger $\rightarrow>$

<owl:NamedIndividual rdf:about="http://www.semanticweb.org/thesis.owl\#Type-Finger"> <rdf:type rdf:resource="http://www.semanticweb.org/thesis.owl\#Type"/>

$<$ RUInstrument rdf:resource="http://www.semanticweb.org/thesis.owl\#Class-BPM"/>

$<$ /owl:NamedIndividual $>$

$<!-$ http://www.semanticweb.org/thesis.owl\#Type-Oral ->

<owl:NamedIndividual rdf:about="http://www.semanticweb.org/thesis.owl\#Type-Oral">

< rdf:type rdf:resource="http://www.semanticweb.org/thesis.owl\#Type"/>

< RUInstrument rdf:resource="http://www.semanticweb.org/thesis.owl\#Class-Therm"/>

</owl:NamedIndividual $>$

$<!-$ http://www.semanticweb.org/thesis.owl\#Type-Tymp ->

<owl:NamedIndividual rdf:about="http://www.semanticweb.org/thesis.owl\#Type-Tymp"> <rdf:type rdf:resource="http://www.semanticweb.org/thesis.owl\#Type"/>

< RUInstrument rdf:resource="http://www.semanticweb.org/thesis.owl\#Class-Therm"/> </owl:NamedIndividual $>$

$<!-$ http://www.semanticweb.org/thesis.owl\#Unit-Critical $\rightarrow>$

<owl:NamedIndividual rdf:about="http://www.semanticweb.org/thesis.owl\#Unit-Critical"> < rdf:type rdf:resource="http://www.semanticweb.org/thesis.owl\#Care-Unit"/>

$<$ RULocation rdf:resource="http://www.semanticweb.org/thesis.owl\#Hospital-H-2"/> $</$ owl:NamedIndividual $>$ 
$<!-$ http://www.semanticweb.org/thesis.owl\#Unit-Intensive $->$

<owl:NamedIndividual rdf:about="http://www.semanticweb.org/thesis.owl\#Unit-Intensive"> < rdf:type rdf:resource="http://www.semanticweb.org/thesis.owl\#Care-Unit"/>

<RULocation rdf:resource="http://www.semanticweb.org/thesis.owl\#Hospital-H-1"/>

$<$ lowl:NamedIndividual $>$

$<$ !- http://www.semanticweb.org/thesis.owl\#Unit-Standard ->

<owl:NamedIndividual rdf:about="http://www.semanticweb.org/thesis.owl\#Unit-Standard">

< rdf:type rdf:resource="http://www.semanticweb.org/thesis.owl\#Care-Unit"/>

<rdf:type rdf:resource="http://www.semanticweb.org/thesis.owl\#StandardCon"/>

$<$ RULocation rdf:resource="http://www.semanticweb.org/thesis.owl\#Hospital-H-1"/>

$<$ lowl:NamedIndividual $>$

$<$ !- http://www.semanticweb.org/thesis.owl\#Unit-Terminal $->$

<owl:NamedIndividual rdf:about="http://www.semanticweb.org/thesis.owl\#Unit-Terminal"> <rdf:type rdf:resource="http://www.semanticweb.org/thesis.owl\#Care-Unit"/>

<RULocation rdf:resource="http://www.semanticweb.org/thesis.owl\#Hospital-H-1"/>

$<$ lowl:NamedIndividual $>$

<!- http://www.semanticweb.org/thesis.owl\#Value-110/70 ->

<owl:NamedIndividual rdf:about="http://www.semanticweb.org/thesis.owl\#Value-1 10/70">

<rdf:type rdf:resource="http://www.semanticweb.org/thesis.owl\#Value"/>

$<$ lowl:NamedIndividual >

$<$ !- http://www.semanticweb.org/thesis.owl\#Value-37.9 ->

<owl:NamedIndividual rdf:about="http://www.semanticweb.org/thesis.owl\#Value-37.9"> <rdf:type rdf:resource="http://www.semanticweb.org/thesis.owl\#Value"/>

$<$ lowl:NamedIndividual $>$

$<$ !- http://www.semanticweb.org/thesis.owl\#Value-38.0 ->

<owl:NamedIndividual rdf:about="http://www.semanticweb.org/thesis.owl\#Value-38.0"> <rdf:type rdf:resource="http://www.semanticweb.org/thesis.owl\#Value"/> 
$<$ lowl:NamedIndividual $>$

$<$ !- http://www.semanticweb.org/thesis.owl\#Value-38.1 ->

<owl:NamedIndividual rdf:about="http://www.semanticweb.org/thesis.owl\#Value-38.1"> <rdf:type rdf:resource="http://www.semanticweb.org/thesis.owl\#Value"/>

$<$ /owl:NamedIndividual $>$

<!- http://www.semanticweb.org/thesis.owl\#Value-38.2 ->

<owl:NamedIndividual rdf:about="http://www.semanticweb.org/thesis.owl\#Value-38.2"> < rdf:type rdf:resource="http://www.semanticweb.org/thesis.owl\#Value"/>

$</$ owl:NamedIndividual $>$

$<$ !- http://www.semanticweb.org/thesis.owl\#Value-38.5 ->

<owl:NamedIndividual rdf:about="http://www.semanticweb.org/thesis.owl\#Value-38.5"> < rdf:type rdf:resource="http://www.semanticweb.org/thesis.owl\#Value"/>

$<$ lowl:NamedIndividual $>$

<!- http://www.semanticweb.org/thesis.owl\#Ward-W-1 ->

<owl:NamedIndividual rdf:about="http://www.semanticweb.org/thesis.owl\#Ward-W-1"> <rdf:type rdf:resource="http://www.semanticweb.org/thesis.owl\#Ward"/> $<$ RULocation rdf:resource="http://www.semanticweb.org/thesis.owl\#Unit-Standard"/> $</$ owl:NamedIndividual $>$

<!- http://www.semanticweb.org/thesis.owl\#Ward-W-2 ->

<owl:NamedIndividual rdf:about="http://www.semanticweb.org/thesis.owl\#Ward-W-2"> <rdf:type rdf:resource="http://www.semanticweb.org/thesis.owl\#Ward"/>

<RULocation rdf:resource="http://www.semanticweb.org/thesis.owl\#Unit-Standard"/> $<$ lowl:NamedIndividual $>$

$<$ !- http://www.semanticweb.org/thesis.owl\#Ward-W-3 ->

<owl:NamedIndividual rdf:about="http://www.semanticweb.org/thesis.owl\#Ward-W-3"> <rdf:type rdf:resource="http://www.semanticweb.org/thesis.owl\#Ward"/> 
$<$ RULocation rdf:resource="http://www.semanticweb.org/thesis.owl\#Unit-Intensive"/> $<$ lowl:NamedIndividual $>$

$<$ !- http://www.semanticweb.org/thesis.owl\#Ward-W-4 ->

<owl:NamedIndividual rdf:about="http://www.semanticweb.org/thesis.owl\#Ward-W-4"> <rdf:type rdf:resource="http://www.semanticweb.org/thesis.owl\#Ward"/>

$<$ RULocation rdf:resource="http://www.semanticweb.org/thesis.owl\#Unit-Terminal"/> $</$ owl:NamedIndividual $>$

$<$ !- http://www.semanticweb.org/thesis.owl\#Ward-W-5 ->

<owl:NamedIndividual rdf:about="http://www.semanticweb.org/thesis.owl\#Ward-W-5"> <rdf:type rdf:resource="http://www.semanticweb.org/thesis.owl\#Ward"/>

$<$ RULocation rdf:resource="http://www.semanticweb.org/thesis.owl\#Unit-Critical"/> $<$ lowl:NamedIndividual $>$

<!- http://www.semanticweb.org/thesis.owl\#Week-Week/33/2011 ->

<owl:NamedIndividual rdf:about="http://www.semanticweb.org/thesis.owl\#WeekWeek/33/2011">

<rdf:type rdf:resource="http://www.semanticweb.org/thesis.owl\#Week"/>

<RUTime rdf:resource="http://www.semanticweb.org/thesis.owl\#Year-2011"/>

$<$ /owl:NamedIndividual $>$

<!- http://www.semanticweb.org/thesis.owl\#Week-Week14/2009 ->

<owl:NamedIndividual rdf:about="http://www.semanticweb.org/thesis.owl\#WeekWeek 14/2009">

<rdf:type rdf:resource="http://www.semanticweb.org/thesis.owl\#Week"/>

$<$ RUTime rdf:resource="http://www.semanticweb.org/thesis.owl\#Year-2009"/>

$<$ lowl:NamedIndividual $>$

<!- http://www.semanticweb.org/thesis.owl\#Week-Week14/2011 ->

<owl:NamedIndividual rdf:about="http://www.semanticweb.org/thesis.owl\#WeekWeek14/2011"> 
< rdf:type rdf:resource="http://www.semanticweb.org/thesis.owl\#Week"/>

<RUTime rdf:resource="http://www.semanticweb.org/thesis.owl\#Year-2011"/> $<$ lowl:NamedIndividual $>$

$<$ !- http://www.semanticweb.org/thesis.owl\#Week-Week18/2009 ->

<owl:NamedIndividual rdf:about="http://www.semanticweb.org/thesis.owl\#WeekWeek 18/2009">

< rdf:type rdf:resource="http://www.semanticweb.org/thesis.owl\#Week"/>

$<$ RUTime rdf:resource="http://www.semanticweb.org/thesis.owl\#Year-2009"/> $<$ lowl:NamedIndividual $>$

<!- http://www.semanticweb.org/thesis.owl\#Week-Week18/2011 ->

<owl:NamedIndividual rdf:about="http://www.semanticweb.org/thesis.owl\#WeekWeek18/2011">

<rdf:type rdf:resource="http://www.semanticweb.org/thesis.owl\#Week"/>

$<$ RUTime rdf:resource="http://www.semanticweb.org/thesis.owl\#Year-2011"/>

$<$ lowl:NamedIndividual $>$

<!- http://www.semanticweb.org/thesis.owl\#Week-Week28/2000 ->

<owl:NamedIndividual rdf:about="http://www.semanticweb.org/thesis.owl\#WeekWeek28/2000">

<rdf:type rdf:resource="http://www.semanticweb.org/thesis.owl\#Week"/>

<RUTime rdf:resource="http://www.semanticweb.org/thesis.owl\#Year-2000"/>

$<$ lowl:NamedIndividual $>$

<!- http://www.semanticweb.org/thesis.owl\#Week-Week28/2010 ->

<owl:NamedIndividual rdf:about="http://www.semanticweb.org/thesis.owl\#WeekWeek28/2010">

<rdf:type rdf:resource="http://www.semanticweb.org/thesis.owl\#Week"/>

$<$ RUTime rdf:resource="http://www.semanticweb.org/thesis.owl\#Year-2010"/>

</owl:NamedIndividual $>$

<!- http://www.semanticweb.org/thesis.owl\#Week-Week30/2011 -> 
<owl:NamedIndividual rdf:about="http://www.semanticweb.org/thesis.owl\#WeekWeek30/2011">

<rdf:type rdf:resource="http://www.semanticweb.org/thesis.owl\#Week"/>

<RUTime rdf:resource="http://www.semanticweb.org/thesis.owl\#Year-2011"/>

$</$ owl:NamedIndividual $>$

<!- http://www.semanticweb.org/thesis.owl\#Week-Week33/2011 ->

<owl:NamedIndividual rdf:about="http://www.semanticweb.org/thesis.owl\#WeekWeek33/2011">

<rdf:type rdf:resource="http://www.semanticweb.org/thesis.owl\#Week"/>

<RUTime rdf:resource="http://www.semanticweb.org/thesis.owl\#Year-2011"/>

$<$ lowl:NamedIndividual $>$

<!- http://www.semanticweb.org/thesis.owl\#Week-week30/2010 ->

<owl:NamedIndividual rdf:about="http://www.semanticweb.org/thesis.owl\#Weekweek30/2010">

<rdf:type rdf:resource="http://www.semanticweb.org/thesis.owl\#Week"/>

<RUTime rdf:resource="http://www.semanticweb.org/thesis.owl\#Year-2010"/>

$<$ lowl:NamedIndividual $>$

<!- http://www.semanticweb.org/thesis.owl\#Year-2000 ->

<owl:NamedIndividual rdf:about="http://www.semanticweb.org/thesis.owl\#Year-2000"> < rdf:type rdf:resource="http://www.semanticweb.org/thesis.owl\#Year"/>

$<$ lowl:NamedIndividual $>$

$<$ !- http://www.semanticweb.org/thesis.owl\# Year-2009 ->

<owl:NamedIndividual rdf:about="http://www.semanticweb.org/thesis.owl\#Year-2009"> <rdf:type rdf:resource="http://www.semanticweb.org/thesis.owl\#Year"/>

$</$ owl:NamedIndividual $>$

$<!-$ http://www.semanticweb.org/thesis.owl\#Year-2010 ->

<owl:NamedIndividual rdf:about="http://www.semanticweb.org/thesis.owl\#Year-2010"> 
<rdf:type rdf:resource="http://www.semanticweb.org/thesis.owl\#Year"/>

$<$ lowl:NamedIndividual $>$

<!- http://www.semanticweb.org/thesis.owl\#Year-2011 ->

<owl:NamedIndividual rdf:about="http://www.semanticweb.org/thesis.owl\#Year-2011">

<rdf:type rdf:resource="http://www.semanticweb.org/thesis.owl\#Year"/>

</owl:NamedIndividual $>$

$</$ rdf:RDF $>$

$<$ !- Generated by the OWL API (version 3.3.1957) http://owlapi.sourceforge.net -> 


\section{Appendix B}

\section{Mappings from the Data Source to the Ontology}

[PrefixDeclaration] : http://www.semanticweb.org/thesis.owl\#

[ClassDeclaration] collection [[

<http://www.semanticweb.org/thesis.owl\#M1Relate> ,

<http://www.semanticweb.org/thesis.owl\#WardCity>,

$<$ http://www.semanticweb.org/thesis.owl\#DayYear>,

<http://www.semanticweb.org/thesis.owl\#NurseHospital >

<http://www.semanticweb.org/thesis.owl\#HourMonth>,

<http://www.semanticweb.org/thesis.owl\#ShiftYear>,

$<$ http://www.semanticweb.org/thesis.owl\#Hospital>,

$<$ http://www.semanticweb.org/thesis.owl\#Holidays $>$,

$<$ http://www.semanticweb.org/thesis.owl\#CityCountry>,

$<$ http://www.semanticweb.org/thesis.owl\#BrandType $>$,

$<$ http://www.semanticweb.org/thesis.owl\#All-Instrument>,

<http://www.semanticweb.org/thesis.owl\#ModelBrand>,

$<$ http://www.semanticweb.org/thesis.owl\#ClosedMonth >

$<$ http://www.semanticweb.org/thesis.owl\#City>,

$<$ http://www.semanticweb.org/thesis.owl\#ManufacturerClass >

$<$ http://www.semanticweb.org/thesis.owl\#NurseCity $>$,

$<$ http://www.semanticweb.org/thesis.owl\#BrandManufacturer $>$, 
$<$ http://www.semanticweb.org/thesis.owl\#Month>, $<$ http://www.semanticweb.org/thesis.owl\#All-Time> <http://www.semanticweb.org/thesis.owl\#Value > $<$ http://www.semanticweb.org/thesis.owl\#StandardCon $>$, $<$ http://www.semanticweb.org/thesis.owl\#WardUnit>, $<$ http://www.semanticweb.org/thesis.owl\#UnitCountry > $<$ http://www.semanticweb.org/thesis.owl\#UnitCity $>$, $<$ http://www.semanticweb.org/thesis.owl\#Manufacturer $>$, $<$ http://www.semanticweb.org/thesis.owl\#ModelType>, $<$ http://www.semanticweb.org/thesis.owl\#Brand >, < http://www.semanticweb.org/thesis.owl\#ModalClass>, <http://www.semanticweb.org/thesis.owl\#Guide> <http://www.semanticweb.org/thesis.owl\#ModelManufacturer >, <http://www.semanticweb.org/thesis.owl\#WeekMonth>, <http://www.semanticweb.org/thesis.owl\#HourWeek>, $<$ http://www.semanticweb.org/thesis.owl\#PW $>$, $<$ http://www.semanticweb.org/thesis.owl\#M1Con>, <http://www.semanticweb.org/thesis.owl\#HourYear>, $<$ http://www.semanticweb.org/thesis.owl\#All-Location>, <http://www.semanticweb.org/thesis.owl\#USSRCon>, $<$ http://www.semanticweb.org/thesis.owl\#Week>, <http://www.semanticweb.org/thesis.owl\#NurseUnit>, <http://www.semanticweb.org/thesis.owl\#Year>, <http://www.semanticweb.org/thesis.owl\#StandardRelate> <http://www.w3.org/2002/07/owl\#Thing >, $<$ http://www.semanticweb.org/thesis.owl\#Day> $<$ http://www.semanticweb.org/thesis.owl\#ManufacturerType >, $<$ http://www.semanticweb.org/thesis.owl\#MonthYear>, $<$ http://www.semanticweb.org/thesis.owl\#Country>, 
<http://www.semanticweb.org/thesis.owl\#Class >, <http://www.semanticweb.org/thesis.owl\#ShiftWeek>, <http://www.semanticweb.org/thesis.owl\#NurseWard>, <http://www.semanticweb.org/thesis.owl\#Inter> $<$ http://www.semanticweb.org/thesis.owl\#WeekDay>, $<$ http://www.semanticweb.org/thesis.owl\#UnitHospital>, $<$ http://www.semanticweb.org/thesis.owl\#WeekYear>, <http://www.semanticweb.org/thesis.owl\#WardHospital>, <http://www.semanticweb.org/thesis.owl\#ShiftMonth>, $<$ http://www.semanticweb.org/thesis.owl\#Care-Unit>, <http://www.semanticweb.org/thesis.owl\#DatesBefore1991>, <http://www.semanticweb.org/thesis.owl\#Before-1991>, $<\mathrm{http}: / /$ www.semanticweb.org/thesis.owl\#Shift>, <http://www.semanticweb.org/thesis.owl\#USSRRelate> $<$ http://www.semanticweb.org/thesis.owl\#BrandClass > , $<$ http://www.semanticweb.org/thesis.owl\#WardCountry>, $<$ http://www.w3.org/2002/07/owl\#Nothing $>$, <http://www.semanticweb.org/thesis.owl\#ShiftDay>, $<$ http://www.semanticweb.org/thesis.owl\#Patient $>$, <http://www.semanticweb.org/thesis.owl\#NurseCountry>, <http://www.semanticweb.org/thesis.owl\#HospitalCountry>, $<$ http://www.semanticweb.org/thesis.owl\#Hour>, <http://www.semanticweb.org/thesis.owl\#TypeClass>, <http://www.semanticweb.org/thesis.owl\#HospitalCity>, $<$ http://www.semanticweb.org/thesis.owl\#DayMonth >, <http://www.semanticweb.org/thesis.owl\#HourDay>, <http://www.semanticweb.org/thesis.owl\#Type>, <http://www.semanticweb.org/thesis.owl\#Ward>, $<$ http://www.semanticweb.org/thesis.owl\#HourShift $>$, 
<http://www.semanticweb.org/thesis.owl\#Model>, $<$ http://www.semanticweb.org/thesis.owl\#Intra $>$, $<$ http://www.semanticweb.org/thesis.owl\#Nurse >

]]

[ObjectPropertyDeclaration] collection [[

$<$ http://www.semanticweb.org/thesis.owl\#RUTime> ,

<http://www.semanticweb.org/thesis.owl\#InvRUInstrument>, <http://www.semanticweb.org/thesis.owl\#RULocation>,

<http://www.semanticweb.org/thesis.owl\#InvHospitalFounded >, <http://www.semanticweb.org/thesis.owl\#InvUnitIns>, <http://www.semanticweb.org/thesis.owl\#PWPatient>, $<$ http://www.semanticweb.org/thesis.owl\#PWValue >,

<http://www.semanticweb.org/thesis.owl\#InvRULocation>

<http://www.semanticweb.org/thesis.owl\#HospitalFounded >, <http://www.semanticweb.org/thesis.owl\#RUInstrument>, <http://www.semanticweb.org/thesis.owl\#UnitIns>, <http://www.semanticweb.org/thesis.owl\#InvRUTime>, <http://www.semanticweb.org/thesis.owl\#PWWard>, $<$ http://www.semanticweb.org/thesis.owl\#RenovationTimes $>$, $<$ http://www.semanticweb.org/thesis.owl\#PWHour >, ]]

[DataPropertyDeclaration] collection [I <http://www.semanticweb.org/thesis.owl\#HospitalType>, <http://www.semanticweb.org/thesis.owl\#HospitalSystem>, ]]

[SourceDeclaration] sourceUri DataSource connectionUrl jdbc:postgresql://localhost:5432/example.thesis username postgres password 1334 


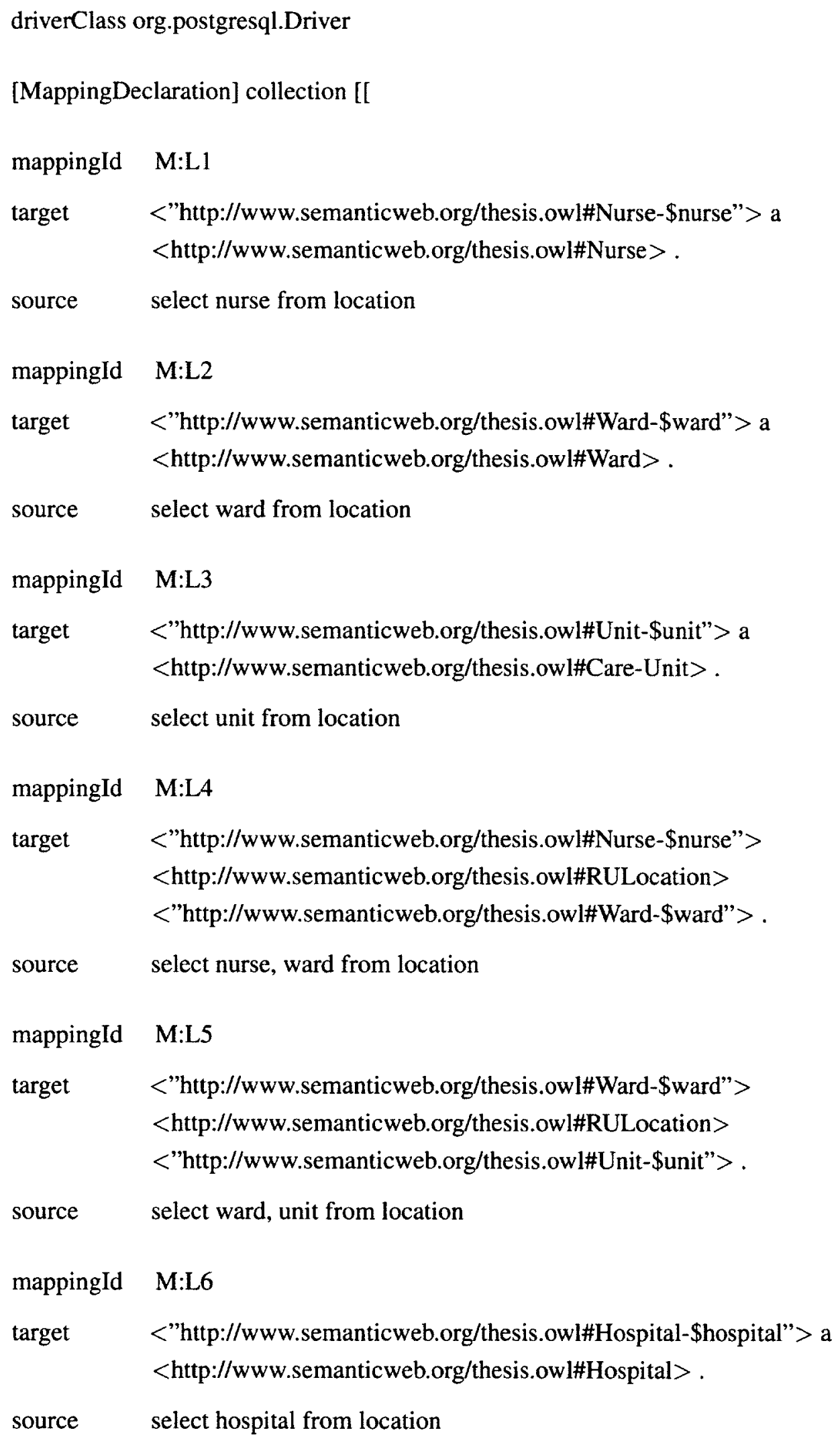




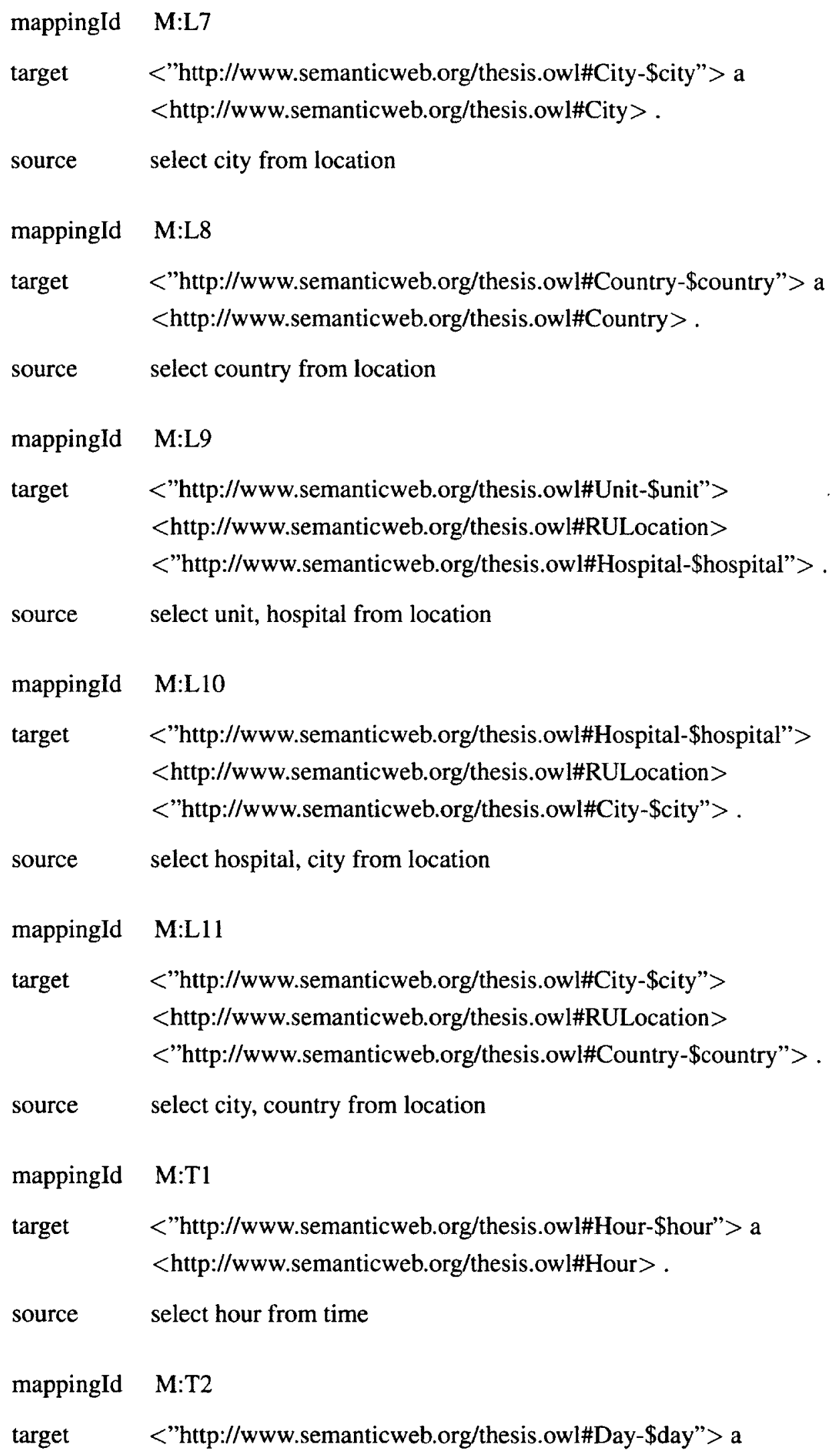


$<$ http://www.semanticweb.org/thesis.owl\#Day > .

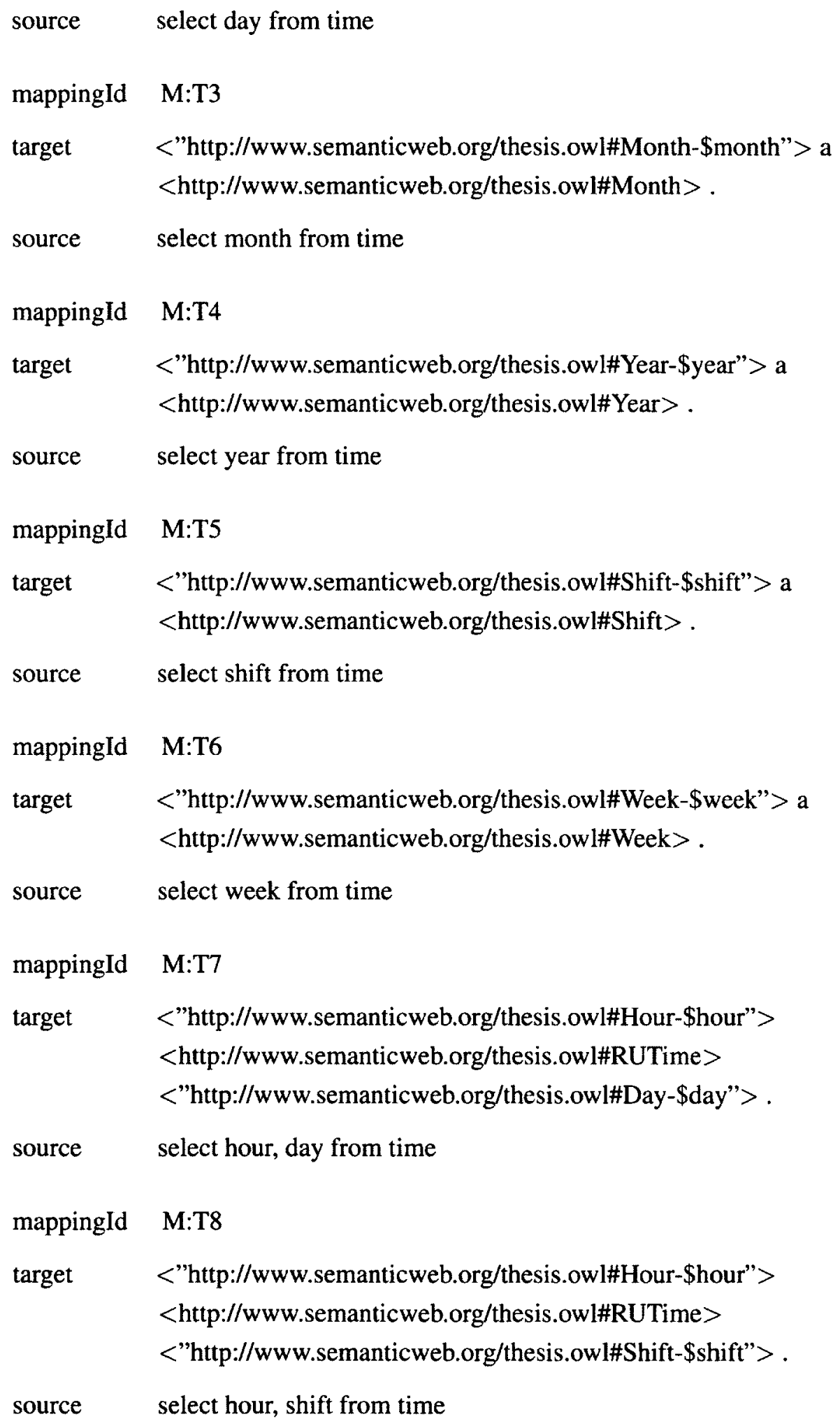




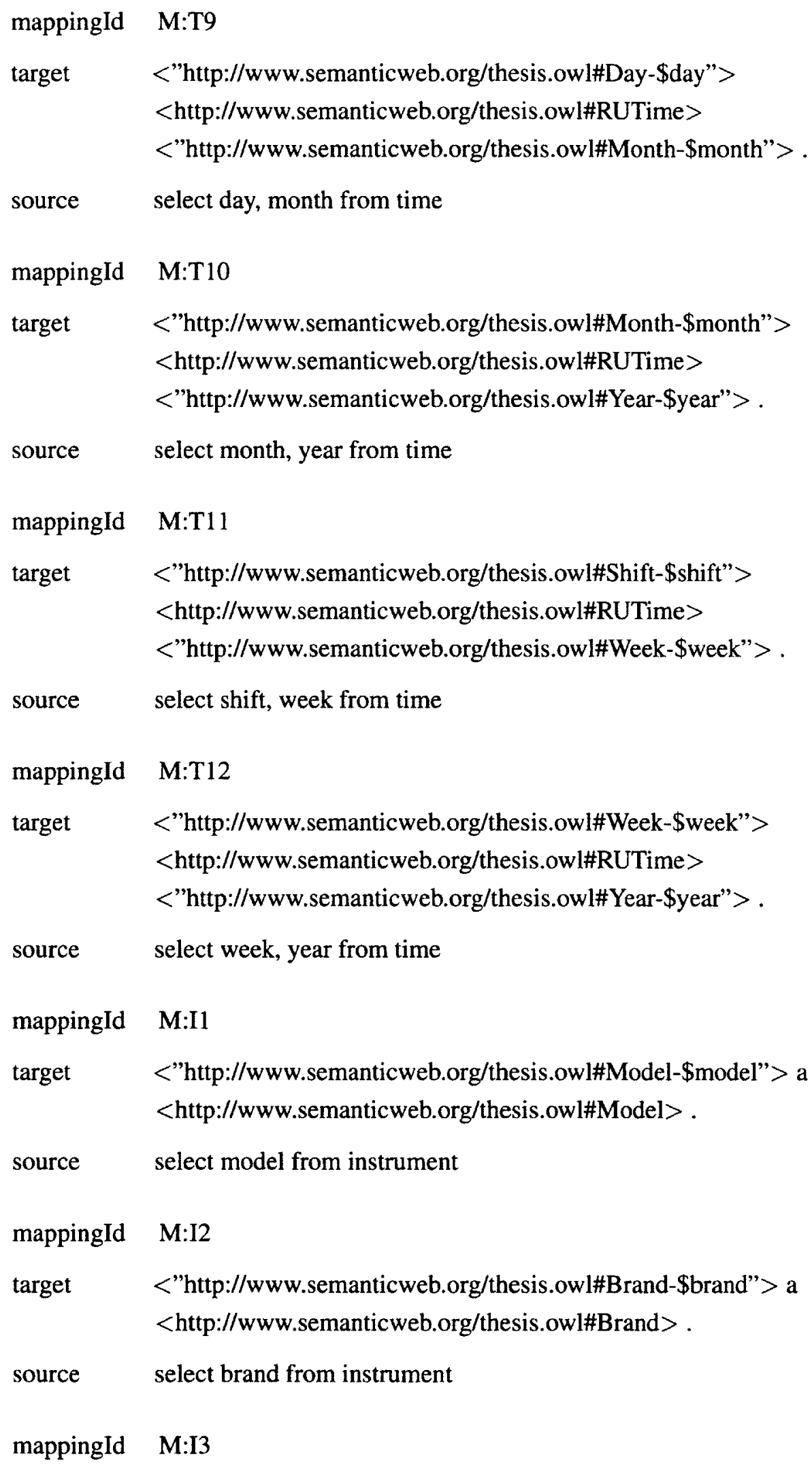




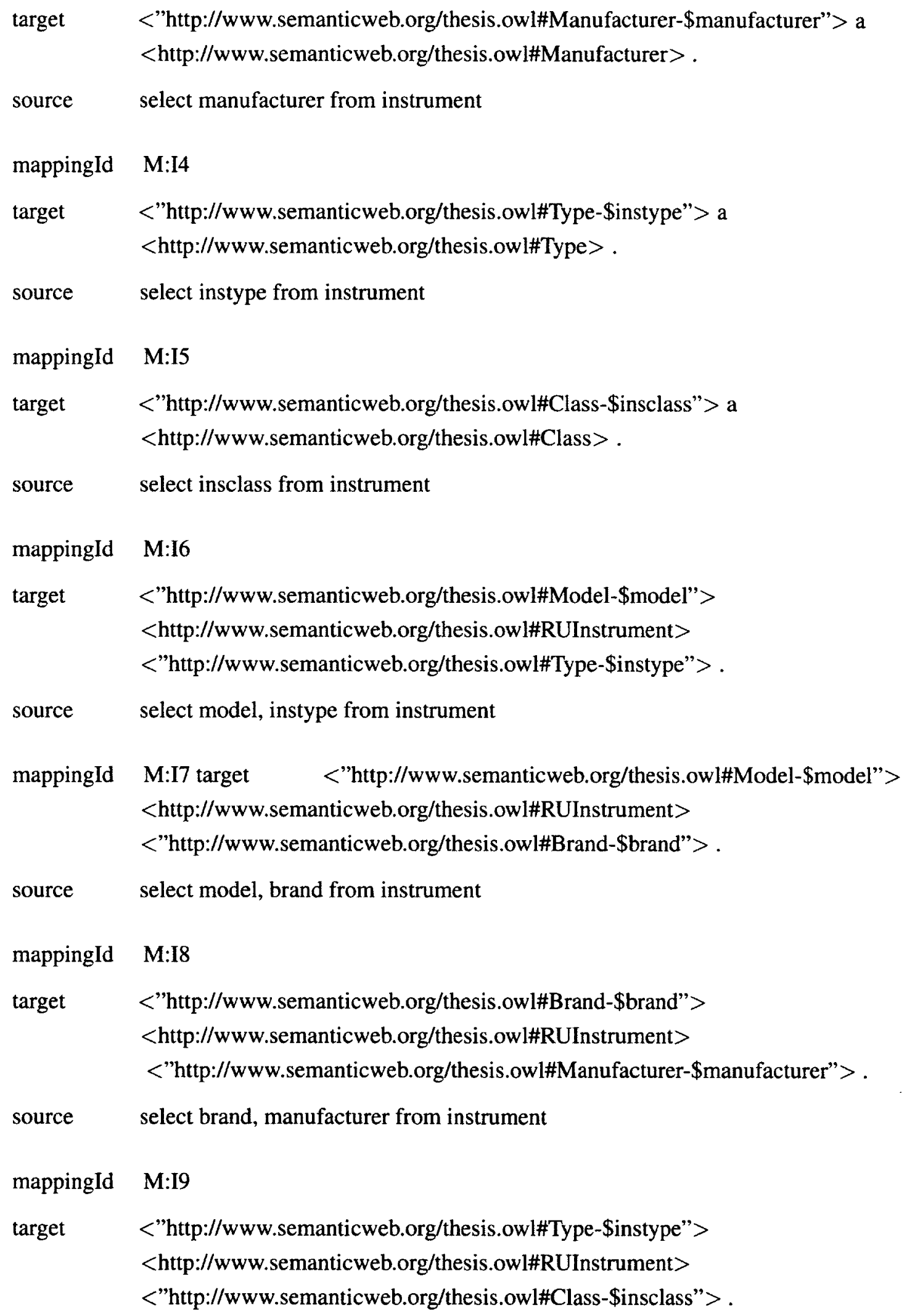




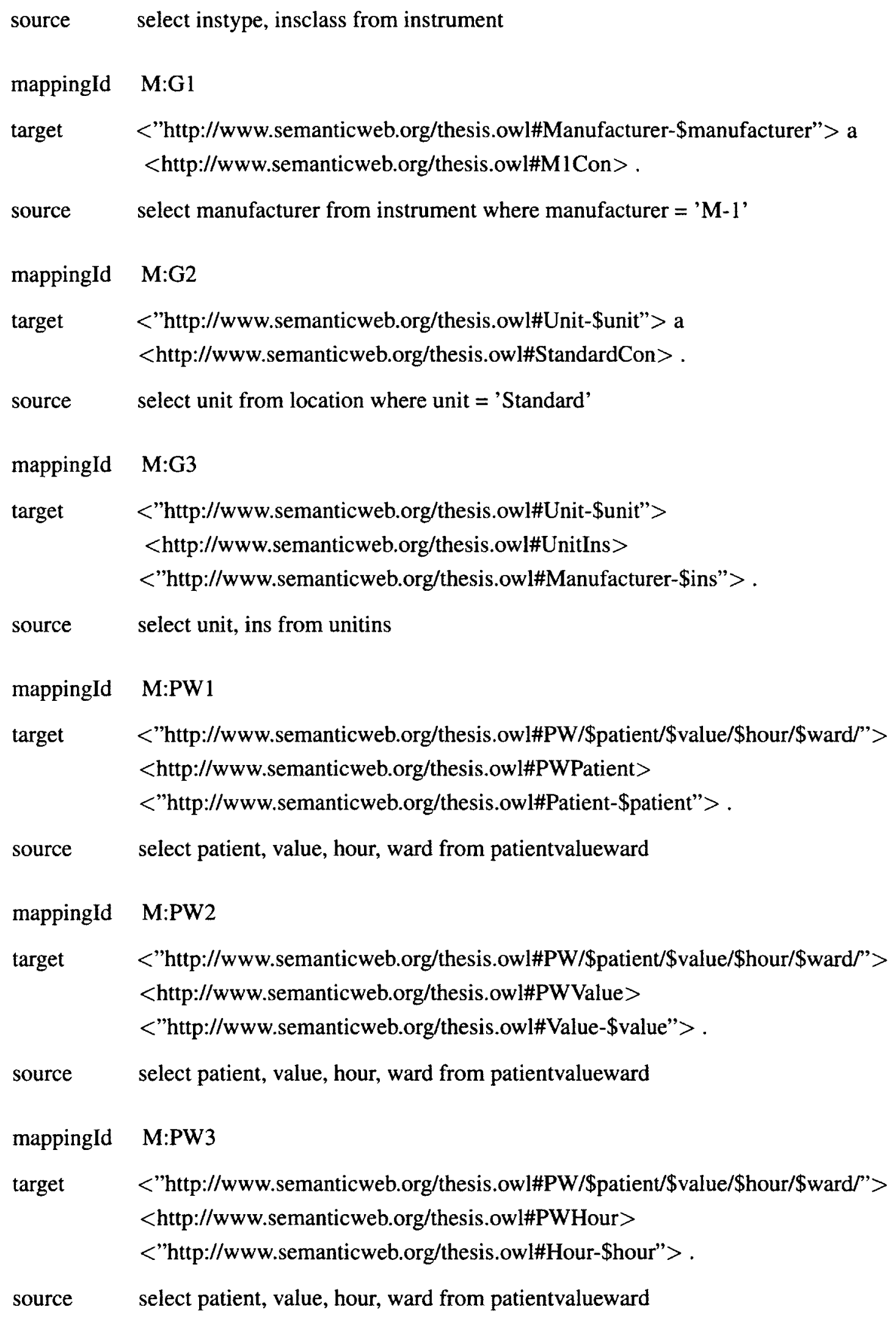




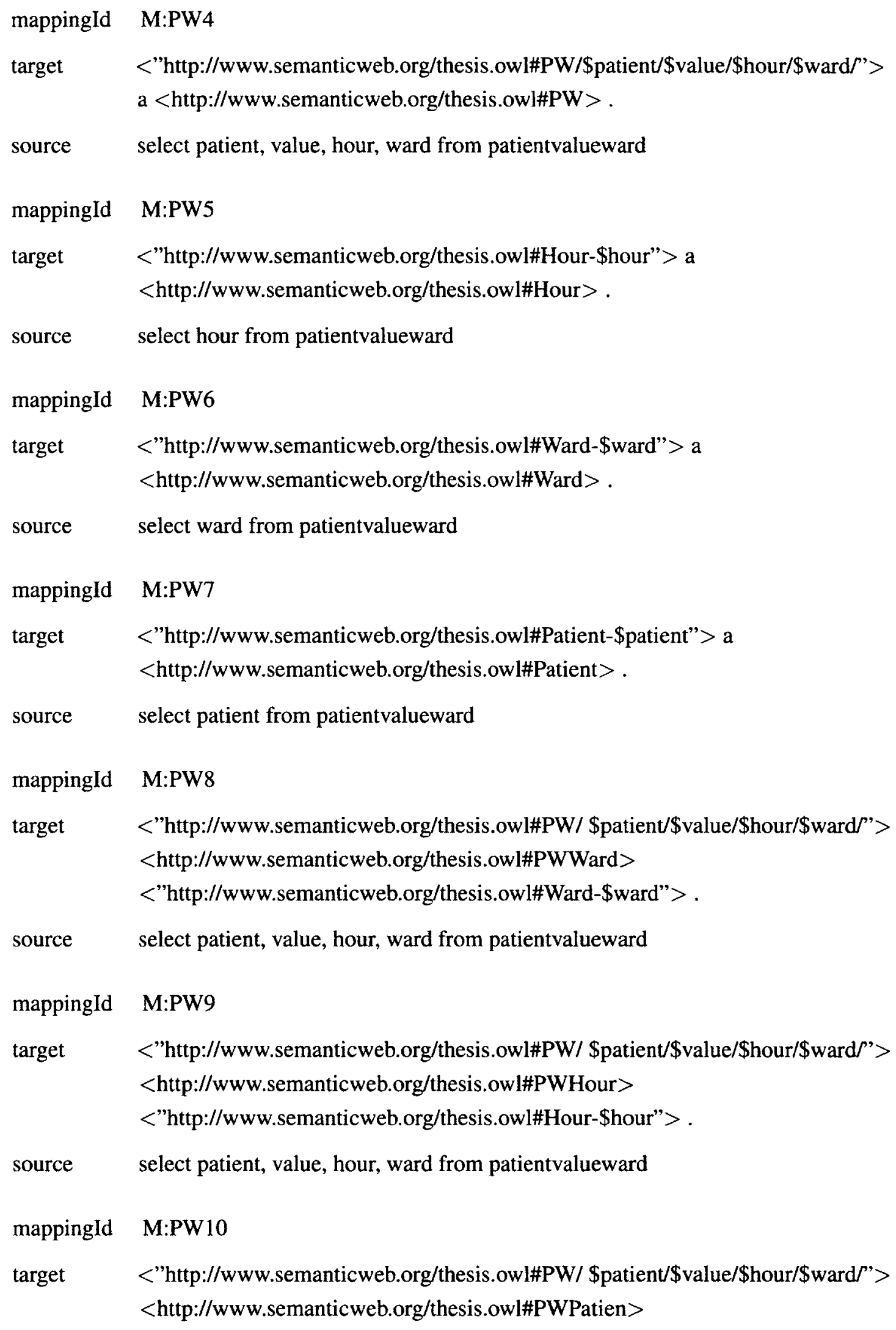


<"http://www.semanticweb.org/thesis.owl\#Patient-\$patient"> .

source select patient, value, hour, ward from patientvalueward

mappingId M:PW11

target <"http://www.semanticweb.org/thesis.owl\#PW/ \$patient/\$value/\$hour/\$ward/> $<$ http://www.semanticweb.org/thesis.owl\#PWValue >

<"http://www.semanticweb.org/thesis.owl\#Value-\$value"> .

source select patient, value, hour, ward from patientvalueward

mappingId M:PW12

target <"http://www.semanticweb.org/thesis.owl\#Value-\$value"> a $<$ http://www.semanticweb.org/thesis.owl\#Value > .

source select value from patientvalueward

mappingId M:Holidays

target <"http://www.semanticweb.org/thesis.owl\#Day-\$day"> a

$<$ http://www.semanticweb.org/thesis.owl\#Holidays $>$.

source select day from workingdayscalendar

mappingId M:ClosedMonth

target <"http://www.semanticweb.org/thesis.owl\#Month-\$month">a $<$ http://www.semanticweb.org/thesis.owl\#ClosedMonth $>$.

source select month from renovationtimes

mappingId M:USSR

target <"http://www.semanticweb.org/thesis.owl\#Country-\$Country"> a $<$ http://www.semanticweb.org/thesis.owl\#USSRCon> .

source select country from location where country = 'USSR'

mappingId M:HospitalFounded

target <"http://www.semanticweb.org/thesis.owl\#Hospital-\$hospital"> $<$ http://www.semanticweb.org/thesis.owl\#HospitalFounded $>$ <"http://www.semanticweb.org/thesis.owl\#Day-\$day"> .

source select hospital, day from hospitalfounded 


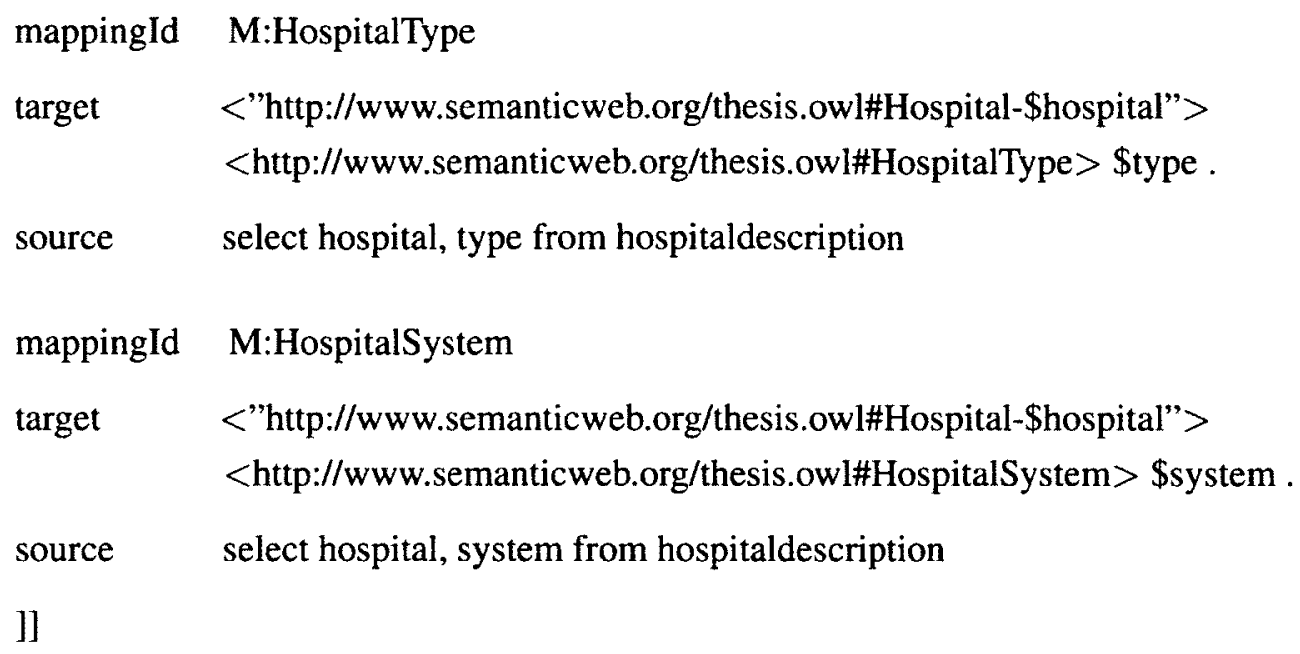

